\title{
Synthesis of Bis-heteroaryls using Grignard Reagents and Pyridylsulfonium Salts
}

\author{
Alexandra M. Horan, Vincent K. Duong, * Eoghan M. McGarrigle* \\ SSPC, the SFI Research Centre for Pharmaceuticals, Centre for Synthesis \& Chemical Biology, UCD \\ School of Chemistry, University College Dublin, Belfield, Dublin 4, Ireland. \\ Supporting Information
}

\section{Contents}

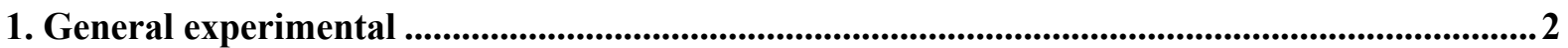

2. Synthesis of sulfides ..........................................................................................................................

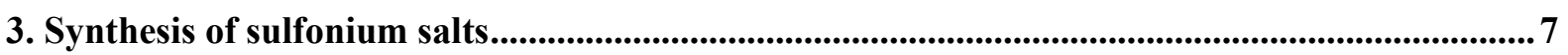

4. Synthesis of bipyridines .....................................................................................................................11

5. Synthesis of Caerulomycin $E$ and $A$...................................................................................................2 27

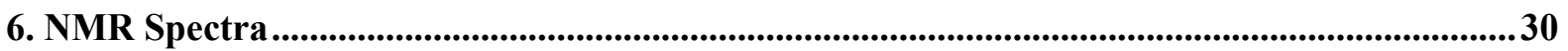

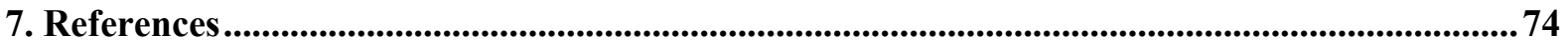




\section{General experimental}

Chemicals were purchased and used without further purification unless otherwise stated. Solvents were dried using a Grubbs-type still, a Pure Solv-400-3-MD solvent purification system supplied by Innovative Technology Inc. design and stored in Strauss flasks over activated $4 \AA$ molecular sieves. Diphenyliodonium trifluoromethanesulfonate was prepared according to literature procedure. ${ }^{1}$ Pyridylsulfides 1ai-1gi and sulfonium salts 1a-1g were prepared as previously described. ${ }^{2}$ $\mathrm{TMPMgCl} \cdot \mathrm{LiCl}$ was prepared according to literature procedure. ${ }^{3} i$-PrMgCl$\bullet \cdot \mathrm{LiCl}$ and $\mathrm{TMPMgCl} \cdot \mathrm{LiCl}$ were titrated prior to use. ${ }^{4}$

Reactions requiring anhydrous conditions were performed under $\mathrm{N}_{2}$; glassware was flame-dried immediately prior to use and allowed to cool under reduced pressure. Reaction monitoring by TLC was performed on Merck pre-coated Kieselgel $60 \mathrm{~F}_{254}$ aluminium plates. Visualization was accomplished under UV light (254 nm). Flash column chromatography (FCC) was performed using either silica gel [Davisil, 230-400 mesh (40-63 $\mu \mathrm{m})$ ] or using a Biotage Isolera ${ }^{\mathrm{TM}}$ UV-VIS Flash Purification System Version 2.3.1 with SNAP Ultra $(25 \mu \mathrm{m})$, SNAP KP-Sil $(50 \mu \mathrm{m})$ or SNAP KP-NH $(50 \mu \mathrm{m})$ prepacked silica cartridges. High-resolution mass spectra were run on a Waters Micromass GCT system or on an Agilent 6546 QTOF system in electrospray ionization mode (ESI). Extracts were concentrated in vacuo using both a rotary evaporator (bath temperatures up to $55^{\circ} \mathrm{C}$ ), and a high vacuum line at room temperature. ${ }^{1} \mathrm{H}$ NMR and ${ }^{13} \mathrm{C}$ NMR spectra were measured in the solvent stated at $300 \mathrm{MHz}, 400 \mathrm{MHz}$ or $500 \mathrm{MHz}$ or $100-126 \mathrm{MHz}$, respectively. ${ }^{19} \mathrm{~F}$ spectra was measured in the solvent stated at $376-$ $470 \mathrm{MHz}$. Chemical shifts $(\delta)$ are quoted in parts per million (ppm) referenced to residual solvent peak (e.g., $\mathrm{CDCl}_{3}:{ }^{1} \mathrm{H}-7.26 \mathrm{ppm}$ and $\left.{ }^{13} \mathrm{C}-77.16 \mathrm{ppm}\right)$ or TMS $\left({ }^{1} \mathrm{H}-0.00 \mathrm{ppm}\right)$ and coupling constants $(J)$ are given in Hertz. Multiplicities are abbreviated as: b (broad), s (singlet), d (doublet), t (triplet), $\mathrm{q}$ (quartet), m (multiplet) or combinations thereof. Assignments were made, where necessary, with the aid of COSY, HSQC, HMBC and NOESY NMR experiments.

\section{Synthesis of sulfides}

General procedure A: 4-methylbenzenethiol (1 equiv.) was added to a round bottomed flask, followed by halopyridine (1 equiv.) and $\mathrm{H}_{2} \mathrm{O}(0.5 \mathrm{M})$. The reaction was heated under reflux for $24 \mathrm{~h}$ (the flask sitting in an aluminium block placed on a stirrer hotplate). Then, the reaction was allowed to cool, followed by extraction with EtOAc. The combined organic layers were washed with water and brine and dried over $\mathrm{Na}_{2} \mathrm{SO}_{4}$. The solvent was evaporated, and the product was isolated by FCC.

General procedure B: 4-methylbenzenethiol (1 equiv.) was added to a crimptop vial followed by halopyridine (1 equiv.) and $\mathrm{K}_{2} \mathrm{CO}_{3}$ (1.1 equiv.) and closed. The vial was evacuated and purged with $\mathrm{N}_{2}$ three times. Anhydrous DMF (0.8-1 M wrt thiol/halopyridine) was added. The reaction was heated to $100{ }^{\circ} \mathrm{C}$ for $24 \mathrm{~h}$ (the flask sitting in an aluminium block placed on a stirrer hotplate). Then the reaction 
was allowed to cool, followed by the addition of water and extraction with EtOAc. The combined organic layers were washed with water and brine and dried over $\mathrm{Na}_{2} \mathrm{SO}_{4}$. Details of purification are given below for each compound.

\section{2-Methyl-6-(p-tolylthio)pyridine 1 hi}<smiles>Cc1ccc(Sc2cccc(C)n2)cc1</smiles>

Prepared from General procedure B with 4-methylbenzenethiol (0.62 g, $5.0 \mathrm{mmol})$, 2-bromo-6methylpyridine $(0.57 \mathrm{~mL}, 5.0 \mathrm{mmol}), \mathrm{K}_{2} \mathrm{CO}_{3}(0.88 \mathrm{~g}, 6.0 \mathrm{mmol})$ and $\mathrm{DMF}(6 \mathrm{~mL})$. The reaction was extracted with EtOAc $(3 \times 15 \mathrm{~mL})$. The combined organic layers were washed with water $(20 \mathrm{~mL})$ and brine $(20 \mathrm{~mL})$ and dried over $\mathrm{Na}_{2} \mathrm{SO}_{4}$. The solvent was evaporated to give pyridylsulfide $\mathbf{1 h i}$ as a clear oil $(1.05 \mathrm{~g}, 98 \%)$.

TLC: $R_{\mathrm{f}}=0.3\left(10 \% \mathrm{Et}_{2} \mathrm{O}\right.$ in pentane $)$.

${ }^{1} \mathrm{H}$ NMR (500 MHz, Chloroform- $d$ ) $\delta 7.50-7.46$ (m, 2H, ArH), $7.28(\mathrm{t}, J=7.8 \mathrm{~Hz}, 1 \mathrm{H}, \mathrm{ArH}), 7.24-$ 7.18 (m, 2H, ArH), 6.81 (d, J=7.6 Hz, 1H, ArH), 6.55 (d, J=8.0 Hz, 1H, ArH), 2.49 (s, 3H, $\mathrm{CH}_{3}$ ), $2.38\left(\mathrm{~s}, 3 \mathrm{H}, \mathrm{CH}_{3}\right) \mathrm{ppm}$.

${ }^{13} \mathrm{C}$ NMR (126 MHz, Chloroform- $d$ ) $\delta 161.9(\mathrm{C}), 158.5(\mathrm{C}), 139.5(\mathrm{C}), 137.0(\mathrm{CH}), 135.4(\mathrm{CH}), 130.5$ $(\mathrm{CH}), 127.6(\mathrm{C}), 119.2(\mathrm{CH}), 117.7(\mathrm{CH}), 24.4\left(\mathrm{CH}_{3}\right), 21.4\left(\mathrm{CH}_{3}\right) \mathrm{ppm}$.

HRMS (ESI-TOF) $m / z$ : [M+H] $]^{+}$Calcd for $\mathrm{C}_{13} \mathrm{H}_{14} \mathrm{NS}: 216.0841$; Found: 216.0844 .

\section{2-(p-Tolylthio)-6-(trifluoromethyl)pyridine 1ii}<smiles>Cc1ccc(Sc2cccc(C(F)(F)F)n2)cc1</smiles>

Prepared from General procedure B with slight modifications, with 4-methylbenzenethiol ( $0.56 \mathrm{~g}, 4.5$ mmol), 2-bromo-6-(trifluoromethyl)pyridine (0.68 g, $3.0 \mathrm{mmol}), \mathrm{K}_{2} \mathrm{CO}_{3}(0.50 \mathrm{~g}, 3.6 \mathrm{mmol})$ and DMF $(1.9 \mathrm{~mL})$. The reaction was heated to $140{ }^{\circ} \mathrm{C}$ for $7 \mathrm{~h}$. The reaction was extracted with EtOAc $(3 \times 20$ $\mathrm{mL})$. The combined organic layers were washed with water $(3 \times 30 \mathrm{~mL})$ and brine $(3 \times 30 \mathrm{~mL})$ and dried over $\mathrm{Na}_{2} \mathrm{SO}_{4}$. The solvent was evaporated and purification by $\mathrm{FCC}\left(5 \% \mathrm{Et}_{2} \mathrm{O}\right.$ in pentane) gave pyridylsulfide 1ii as a white solid (0.52 g, 64\%).

TLC: $R_{\mathrm{f}}=0.50\left(5 \% \mathrm{Et}_{2} \mathrm{O}\right.$ in pentane $)$.

${ }^{1} \mathrm{H}$ NMR (500 MHz, Chloroform- $d$ ) $\delta 7.57(\mathrm{t}, J=7.9 \mathrm{~Hz}, 1 \mathrm{H}), 7.52(\mathrm{~d}, J=8.2 \mathrm{~Hz}, 2 \mathrm{H}), 7.33(\mathrm{~d}, J=7.7$ $\mathrm{Hz}, 1 \mathrm{H}), 7.28(\mathrm{~d}, J=7.8 \mathrm{~Hz}, 2 \mathrm{H}), 6.93(\mathrm{~d}, J=8.1 \mathrm{~Hz}, 1 \mathrm{H}), 2.43\left(\mathrm{~s}, 3 \mathrm{H}, \mathrm{CH}_{3}\right)$. 
${ }^{13} \mathrm{C}$ NMR (101 MHz, Chloroform- $d$ ) $\delta 164.3(\mathrm{C}), 147.9$ (q, $\left.J=34.8 \mathrm{~Hz}, C_{C F}\right), 140.3(\mathrm{C}), 137.7(\mathrm{CH})$, $135.7(\mathrm{CH}), 130.9(\mathrm{CH}), 126.1(\mathrm{C}), 123.2(\mathrm{CH}), 121.3\left(\mathrm{~d}, J=274.3 \mathrm{~Hz}, \mathrm{CF}_{3}\right), 116.1(\mathrm{q}, J=2.7 \mathrm{~Hz}$, $\mathrm{CH}), 21.5\left(\mathrm{CH}_{3}\right)$.

${ }^{19} \mathrm{~F}$ NMR $(282 \mathrm{MHz}$, Chloroform- $d$ ) $\delta$-68.2.

HRMS (ESI-TOF) $m / z$ : [M+H] $]^{+}$Calcd for $\mathrm{C}_{13} \mathrm{H}_{11} \mathrm{~F}_{3} \mathrm{NS}: 270.0559$; Found: 270.0561 .

\section{6-(p-Tolylthio)nicotinonitrile $1 \mathrm{ji}$}<smiles>Cc1ccc(Sc2ccc(C#N)cn2)cc1</smiles>

Prepared from General procedure B with slight modifications, with 4-methylbenzenethiol (1.65 g, 13.3 mmol), 2-bromo-5-cyanopyridine (2.03 g, $11.1 \mathrm{mmol}), \mathrm{K}_{2} \mathrm{CO}_{3}(2.31 \mathrm{~g}, 16.7 \mathrm{mmol}$ ) and DMF (5.6 mL). The reaction was heated to $140{ }^{\circ} \mathrm{C}$ for $16 \mathrm{~h}$. The reaction was extracted with EtOAc $(3 \times 20 \mathrm{~mL})$. The combined organic layers were washed with water $(3 \times 30 \mathrm{~mL})$ and brine $(3 \times 30 \mathrm{~mL})$ and dried over $\mathrm{Na}_{2} \mathrm{SO}_{4}$. The solvent was evaporated and purification by $\mathrm{FCC}\left(20 \% \mathrm{Et}_{2} \mathrm{O}\right.$ in pentane) gave pyridylsulfide $1 \mathbf{j i}$ as a white solid $(1.60 \mathrm{~g}, 64 \%)$.

TLC: $R_{\mathrm{f}}=0.37\left(20 \% \mathrm{Et}_{2} \mathrm{O}\right.$ in pentane).

${ }^{1} \mathrm{H}$ NMR (400 MHz, Chloroform- $d$ ) $\delta 8.62(\mathrm{~d}, J=2.1 \mathrm{~Hz}, 1 \mathrm{H}), 7.61(\mathrm{dd}, J=8.5,2.3 \mathrm{~Hz}, 1 \mathrm{H}), 7.48(\mathrm{~d}$, $J=8.0 \mathrm{~Hz}, 2 \mathrm{H}), 7.29(\mathrm{~d}, J=7.8 \mathrm{~Hz}, 2 \mathrm{H}), 6.87(\mathrm{~d}, J=8.4 \mathrm{~Hz}, 1 \mathrm{H}), 2.42\left(\mathrm{~s}, 3 \mathrm{H}, \mathrm{CH}_{3}\right)$.

${ }^{13} \mathrm{C}$ NMR (101 MHz, Chloroform- $d$ ) $\delta 168.7,152.2,140.9,138.9,135.7,131.0,125.0,120.0,117.0$, 105.0, $21.5\left(\mathrm{CH}_{3}\right)$.

Spectra were consistent with literature data. ${ }^{5}$

\section{5-Bromo-2-(p-tolylthio)pyridine 1 ki}<smiles>Cc1ccc(Sc2ccc(Br)cn2)cc1</smiles>

Prepared from General procedure B with slight modifications, with 4-methylbenzenethiol $(0.37 \mathrm{~g}, 3.0$ mmol), 2,5-dibromopyridine (0.71 g, $3.0 \mathrm{mmol}), \mathrm{K}_{2} \mathrm{CO}_{3}(0.5 \mathrm{~g}, 3.6 \mathrm{mmol})$ and DMF (1.9 mL). The reaction was heated to $140{ }^{\circ} \mathrm{C}$ for $7 \mathrm{~h}$. The reaction was extracted with EtOAc $(3 \times 20 \mathrm{~mL})$. The combined organic layers were washed with water $(3 \times 30 \mathrm{~mL})$ and brine $(3 \times 30 \mathrm{~mL})$ and dried over $\mathrm{Na}_{2} \mathrm{SO}_{4}$. The solvent was evaporated and purification by $\mathrm{FCC}\left(5 \% \mathrm{Et}_{2} \mathrm{O}\right.$ in pentane) gave pyridylsulfide 1ki as a white solid $(0.36 \mathrm{~g}, 43 \%)$.

TLC: $R_{\mathrm{f}}=0.36\left(5 \% \mathrm{Et}_{2} \mathrm{O}\right.$ in pentane $)$. 
${ }^{1} \mathrm{H}$ NMR (400 MHz, Chloroform- $d$ ) $\delta 8.45(\mathrm{~d}, J=2.4 \mathrm{~Hz}, 1 \mathrm{H}), 7.52(\mathrm{dd}, J=8.6,2.4 \mathrm{~Hz}, 1 \mathrm{H}), 7.47$ (d, $J=7.9 \mathrm{~Hz}, 2 \mathrm{H}), 7.24(\mathrm{~d}, J=7.8 \mathrm{~Hz}, 2 \mathrm{H}), 6.73(\mathrm{dd}, J=8.6,0.6 \mathrm{~Hz}, 1 \mathrm{H}), 2.40\left(\mathrm{~s}, 3 \mathrm{H}, \mathrm{CH}_{3}\right)$.

${ }^{13} \mathrm{C}$ NMR (101 MHz, Chloroform-d) $\delta 161.1(\mathrm{C}), 150.4(\mathrm{CH}), 140.0(\mathrm{C}), 139.2(\mathrm{CH}), 135.4(\mathrm{CH}), 130.7$ $(\mathrm{CH}), 126.9(\mathrm{C}), 122.3(\mathrm{CH}), 116.3(\mathrm{C}), 21.5\left(\mathrm{CH}_{3}\right)$.

HRMS (ESI-TOF) $\mathrm{m} / \mathrm{z}$ : $[\mathrm{M}+\mathrm{H}]^{+}$Calcd for $\mathrm{C}_{12} \mathrm{H}_{11} \mathrm{BrNS}$ : 279.9790, 281.9770; Found: 279.9792, 281.9771 .

\section{5-Chloro-2-(p-tolylthio)pyridine 1 li}<smiles>Cc1ccc(Sc2ccc(Cl)cn2)cc1</smiles>

Prepared from General procedure B with slight modifications, with 4-methylbenzenethiol $(0.5 \mathrm{~g}, 400$ mmol), 2-bromo-5-chloropyridine (0.77 g, $4.0 \mathrm{mmol}), \mathrm{K}_{2} \mathrm{CO}_{3}(0.66 \mathrm{~g}, 4.8 \mathrm{mmol})$ and $\mathrm{DMF}(2.5 \mathrm{~mL})$. The reaction was heated to $140{ }^{\circ} \mathrm{C}$ for $7 \mathrm{~h}$. The reaction was extracted with EtOAc $(3 \times 20 \mathrm{~mL})$. The combined organic layers were washed with water $(3 \times 30 \mathrm{~mL})$ and brine $(3 \times 30 \mathrm{~mL})$ and dried over $\mathrm{Na}_{2} \mathrm{SO}_{4}$. The solvent was evaporated and purification by $\mathrm{FCC}\left(5 \% \mathrm{Et}_{2} \mathrm{O}\right.$ in pentane) gave pyridylsulfide 1 li as a white solid $(0.75 \mathrm{~g}, 79 \%)$.

TLC: $R_{\mathrm{f}}=0.23\left(5 \% \mathrm{Et}_{2} \mathrm{O}\right.$ in pentane).

${ }^{1} \mathrm{H}$ NMR (400 MHz, Chloroform- $d$ ) $\delta 8.37-8.36(\mathrm{~m}, 1 \mathrm{H}), 7.47(\mathrm{~d}, J=8.1 \mathrm{~Hz}, 2 \mathrm{H}), 7.39$ (dd, $J=8.6$, $2.7 \mathrm{~Hz}, 1 \mathrm{H}), 7.24(\mathrm{~d}, J=8.2 \mathrm{~Hz}, 2 \mathrm{H}), 6.79$ (dd, $J=8.6,0.6 \mathrm{~Hz}, 1 \mathrm{H}), 2.40$ (s, 3H, $\mathrm{CH}_{3}$ ).

${ }^{13} \mathrm{C}$ NMR (101 MHz, Chloroform- $d$ ) $\delta 160.5(\mathrm{C}), 148.3(\mathrm{CH}), 139.9(\mathrm{C}), 136.5(\mathrm{CH}), 135.3(\mathrm{CH}), 130.7$ $(\mathrm{CH}), 128.1(\mathrm{C}), 127.0(\mathrm{C}), 121.8(\mathrm{CH}), 21.5\left(\mathrm{CH}_{3}\right)$.

HRMS (ESI-TOF) $\mathrm{m} / \mathrm{z}$ : $[\mathrm{M}+\mathrm{H}]^{+}$Calcd for $\mathrm{C}_{12} \mathrm{H}_{11} \mathrm{ClNS}$ : 236.0295, 238.0266; Found: 236.0297, 238.0265 .

\section{2-(p-Tolylthio)pyrimidine $1 \mathrm{mi}$}<smiles>Cc1ccc(Sc2ncccn2)cc1</smiles>

Prepared from General procedure B with slight modifications, with 4-methylbenzenethiol $(1.91 \mathrm{~g}, 15.4$ mmol), 2-bromopyrimidine (2.04 g, $12.8 \mathrm{mmol}), \mathrm{K}_{2} \mathrm{CO}_{3}(2.65 \mathrm{~g}, 19.2 \mathrm{mmol})$ and DMF (6.4 mL). The reaction was heated to $140{ }^{\circ} \mathrm{C}$ for $7 \mathrm{~h}$. The reaction was extracted with EtOAc $(3 \times 20 \mathrm{~mL})$. The combined organic layers were washed with water $(3 \times 30 \mathrm{~mL})$ and brine $(3 \times 30 \mathrm{~mL})$ and dried over $\mathrm{Na}_{2} \mathrm{SO}_{4}$. The solvent was evaporated and purification by $\mathrm{FCC}\left(20 \% \mathrm{Et}_{2} \mathrm{O}\right.$ in pentane $)$ gave pyridylsulfide $1 \mathrm{mi}$ as a white solid $(1.81 \mathrm{~g}, 70 \%)$. 
TLC: $R_{\mathrm{f}}=0.25\left(20 \% \mathrm{Et}_{2} \mathrm{O}\right.$ in pentane).

${ }^{1} \mathrm{H}$ NMR (400 MHz, Chloroform- $d$ ) $\delta 8.46(\mathrm{~d}, J=5.0 \mathrm{~Hz}, 2 \mathrm{H}), 7.50(\mathrm{~d}, J=8.1 \mathrm{~Hz}, 2 \mathrm{H}), 7.23(\mathrm{~d}, J=7.7$ $\mathrm{Hz}, 2 \mathrm{H}), 6.93(\mathrm{t}, J=4.9 \mathrm{~Hz}, 1 \mathrm{H}), 2.38\left(\mathrm{~s}, 3 \mathrm{H}, \mathrm{CH}_{3}\right)$.

${ }^{13} \mathrm{C}$ NMR (101 MHz, Chloroform-d) $\delta 173.4(\mathrm{C}), 157.7(\mathrm{CH}), 139.7(\mathrm{C}), 135.4(\mathrm{CH}), 130.3(\mathrm{CH}), 125.9$ (C), $117.0(\mathrm{CH}), 21.5\left(\mathrm{CH}_{3}\right)$.

HRMS (ESI-TOF) $m / z$ : [M+H] $]^{+}$Calcd for $\mathrm{C}_{11} \mathrm{H}_{11} \mathrm{~N}_{2} \mathrm{~S}: 203.0637$; Found: 203.0638 . 


\section{Synthesis of sulfonium salts}

General procedure C: Sulfide (1.1 equiv.), $\mathrm{Ph}_{2} \operatorname{IOTf}\left(1.0\right.$ equiv.) and $\mathrm{Cu}(\mathrm{OTf})_{2}(5 \mathrm{~mol} \%)$ were added to a crimptop vial and sealed. The vial was evacuated and purged with $\mathrm{N}_{2}$ three times. Dry DCE (0.6 M) was added to the vial and the reaction was heated to $95^{\circ} \mathrm{C}$ for $16 \mathrm{~h}$ (the vial sitting in an aluminium block placed on a stirrer hotplate). Then the reaction was allowed to cool and sat. aq. $\mathrm{NH}_{4} \mathrm{Cl}(20 \mathrm{~mL})$ was added. The product was extracted using $\mathrm{CH}_{2} \mathrm{Cl}_{2}(3 \times 20 \mathrm{~mL})$ and washed with $\mathrm{H}_{2} \mathrm{O}(2 \times 20 \mathrm{~mL})$ and brine $(20 \mathrm{~mL})$. The solvent was evaporated and product was isolated by FCC.

(6-Methylpyridin-2-yl)(phenyl)(p-tolyl)sulfonium trifluoromethanesulfonate $1 \mathrm{~h}$<smiles>Cc1ccc([S+](c2ccccc2)c2cccc(C)n2)cc1</smiles>

Product $1 \mathbf{h}$ was synthesised via General procedure $\mathrm{C}$ with sulfide 1 hi $(1.0 \mathrm{~g}, 4.6 \mathrm{mmol}), \mathrm{Ph}_{2} \mathrm{IOTf}(1.81$ g, $4.2 \mathrm{mmol})$ and $\mathrm{Cu}(\mathrm{OTf})_{2}(76 \mathrm{mg}, 0.21 \mathrm{mmol})$. Purification by $\mathrm{FCC}\left(5 \% \mathrm{MeOH}\right.$ in $\left.\mathrm{CH}_{2} \mathrm{Cl}_{2}\right)$ gave sulfonium salt $\mathbf{1 h}$ as a yellow oil (575 $\mathrm{mg}, 31 \%)$.

${ }^{1} \mathrm{H}$ NMR (500 MHz, Chloroform- $d$ ) $\delta 8.13-8.09$ (m, 1H), 7.97 (t, $\left.J=7.8 \mathrm{~Hz}, 1 \mathrm{H}\right), 7.91-7.85$ (m, 2H), $7.84-7.79(\mathrm{~m}, 2 \mathrm{H}), 7.77-7.69(\mathrm{~m}, 1 \mathrm{H}), 7.69-7.63(\mathrm{~m}, 2 \mathrm{H}), 7.52-7.44(\mathrm{~m}, 3 \mathrm{H}), 2.60(\mathrm{~s}, 3 \mathrm{H})$, $2.46(\mathrm{~s}, 3 \mathrm{H})$.

${ }^{13} \mathrm{C}$ NMR (126 MHz, Chloroform- $d$ ) $\delta 162.8(\mathrm{C}), 146.4(\mathrm{C}), 146.1(\mathrm{CH}), 140.7(\mathrm{CH}), 134.5(\mathrm{CH}), 132.1$ $(\mathrm{CH}), 132.0(\mathrm{CH}), 131.6(\mathrm{CH}), 131.3(\mathrm{CH}), 128.5(\mathrm{C}), 126.9(\mathrm{CH}), 125.4(\mathrm{C}), 121.0(\mathrm{C}), 24.5\left(\mathrm{CH}_{3}\right)$, $21.9\left(\mathrm{CH}_{3}\right)$. Peak for $\mathrm{SO}_{3} \mathrm{CF}_{3}$ not observed.

HRMS (ESI ${ }^{+}$): [M-OTf] ${ }^{+}$Calcd for $\mathrm{C}_{19} \mathrm{H}_{18} \mathrm{NS}^{+}:$292.1154; found: 292.1156 .

\section{Phenyl(p-tolyl)(6-(trifluoromethyl)pyridin-2-yl)sulfonium trifluoromethanesulfonate 1i}<smiles>Cc1ccc([S+](c2ccccc2)c2cccc(C(F)(F)F)n2)cc1</smiles>

Product 1i was synthesised via General procedure C with sulfide 1ii (0.65 g, $2.42 \mathrm{mmol}), \mathrm{Ph}_{2} \mathrm{IOTf}(1.04$ g, $2.42 \mathrm{mmol}$ ) and $\mathrm{Cu}(\mathrm{OTf})_{2}(43 \mathrm{mg}, 0.12 \mathrm{mmol})$. Purification by $\mathrm{FCC}\left(10 \% \mathrm{MeOH}\right.$ in $\left.\mathrm{CH}_{2} \mathrm{Cl}_{2}\right)$ gave sulfonium salt $1 \mathbf{i}$ as a brown oil $(1.03 \mathrm{~g}, 86 \%)$.

TLC: $R_{\mathrm{f}}=0.30\left(10 \% \mathrm{MeOH}\right.$ in $\left.\mathrm{CH}_{2} \mathrm{Cl}_{2}\right)$. 
${ }^{1} \mathrm{H}$ NMR (400 MHz, Chloroform- $d$ ) $\delta 8.50(\mathrm{~d}, J=8.1 \mathrm{~Hz}, 1 \mathrm{H}), 8.37$ (t, $\left.J=8.0 \mathrm{~Hz}, 1 \mathrm{H}\right), 8.02(\mathrm{~d}, J=7.8$ $\mathrm{Hz}, 1 \mathrm{H}), 7.86$ (dd, $J=7.7,1.7 \mathrm{~Hz}, 2 \mathrm{H}), 7.81$ (d, $J=8.4 \mathrm{~Hz}, 2 \mathrm{H}), 7.78-7.69$ (m, 1H), $7.69-7.61$ (m, $2 \mathrm{H}), 7.47(\mathrm{~d}, J=8.2 \mathrm{~Hz}, 2 \mathrm{H}), 2.43\left(\mathrm{~s}, 3 \mathrm{H}, \mathrm{CH}_{3}\right)$.

${ }^{13} \mathrm{C}$ NMR (101 MHz, Chloroform- $d$ ) $\delta 150.2$ (q, $J=37.0 \mathrm{~Hz}, C_{C F}$ ), $147.6(\mathrm{C}), 147.1(\mathrm{C}), 143.3(\mathrm{CH})$, 135.0 (CH), $132.3(\mathrm{CH}), 132.0(2 \times \mathrm{CH}), 131.6(\mathrm{CH}), 131.5(\mathrm{CH}), 125.2$ (q, J=2.4 Hz, CH), $124.4(\mathrm{C})$, 120.8 (q, $J=320.8 \mathrm{~Hz}, \mathrm{OTf}), 120.2$ (q, $\left.J=274.9 \mathrm{~Hz}, \mathrm{CF}_{3}\right), 119.9(\mathrm{C}), 21.7\left(\mathrm{CH}_{3}\right)$.

${ }^{19}$ F NMR (376 MHz, Chloroform- $d$ ) $\delta-68.1,-78.3$.

HRMS (ESI-TOF) $m / z$ : [M-OTf] ${ }^{+}$Calcd for $\mathrm{C}_{19} \mathrm{H}_{15} \mathrm{~F}_{3} \mathrm{NS}$ : 346.0872; Found: 346.0874.

\section{(5-Cyanopyridin-2-yl)(phenyl)(p-tolyl)sulfonium trifluoromethanesulfonate $1 \mathrm{j}$}

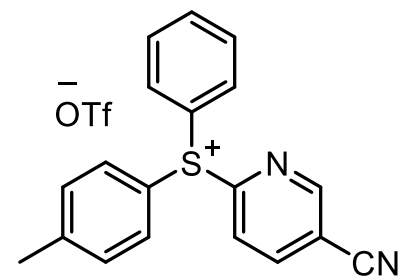

Product $\mathbf{1 j}$ was synthesised via General procedure $\mathrm{C}$ with sulfide $\mathbf{1 j i}$ (1.52 g, $6.73 \mathrm{mmol}$ ), $\mathrm{Ph}_{2} \mathrm{IOTf}(2.90$ $\mathrm{g}, 6.73 \mathrm{mmol})$ and $\mathrm{Cu}(\mathrm{OTf})_{2}(122 \mathrm{mg}, 0.34 \mathrm{mmol})$. Purification by $\mathrm{FCC}\left(10 \% \mathrm{MeOH}\right.$ in $\left.\mathrm{CH}_{2} \mathrm{Cl}_{2}\right)$ gave sulfonium salt $1 \mathrm{j}$ as a brown oil (1.63 $\mathrm{g}, 53 \%)$.

TLC: $R_{\mathrm{f}}=0.36\left(10 \% \mathrm{MeOH}\right.$ in $\left.\mathrm{CH}_{2} \mathrm{Cl}_{2}\right)$.

${ }^{1} \mathrm{H}$ NMR $(500 \mathrm{MHz}$, Chloroform- $d) \delta 8.92(\mathrm{~d}, J=2.1 \mathrm{~Hz}, 1 \mathrm{H}), 8.51(\mathrm{~d}, J=8.2 \mathrm{~Hz}, 1 \mathrm{H}), 8.35(\mathrm{dd}, J=$ 8.3, 2.3 Hz, 1H), 7.89 (d, $J=8.0 \mathrm{~Hz}, 2 \mathrm{H}), 7.83$ (d, $J=8.3 \mathrm{~Hz}, 2 \mathrm{H}), 7.76$ (t, $J=7.5 \mathrm{~Hz}, 1 \mathrm{H}), 7.68$ (t, $J=$ $7.8 \mathrm{~Hz}, 2 \mathrm{H}), 7.50(\mathrm{~d}, J=8.2 \mathrm{~Hz}, 2 \mathrm{H}), 2.47\left(\mathrm{~s}, 3 \mathrm{H}, \mathrm{CH}_{3}\right)$.

${ }^{13} \mathrm{C}$ NMR (126 MHz, Chloroform- $d$ ) $\delta 153.8(\mathrm{CH}), 151.0(\mathrm{C}), 147.3(\mathrm{C}), 144.1(\mathrm{CH}), 135.1(\mathrm{CH}), 132.5$ (CH), $132.3(\mathrm{CH}), 132.0(\mathrm{CH}), 131.7(\mathrm{CH}), 129.5(\mathrm{CH}), 124.0(\mathrm{C}), 120.8$ (q, J=320.3 Hz, OTf), 119.6 (C), $114.7(\mathrm{CN}), 114.5(\mathrm{CCN}), 21.9\left(\mathrm{CH}_{3}\right)$.

${ }^{19} \mathrm{~F}$ NMR (470 MHz, Chloroform- $\left.d\right) \delta-78.3$.

HRMS (ESI-TOF) m/z: [M-OTf] ${ }^{+}$Calcd for $\mathrm{C}_{19} \mathrm{H}_{15} \mathrm{~N}_{2} \mathrm{~S}$ : 303.0950; Found: 303.0954 . 


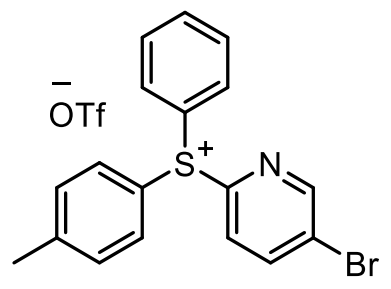

Product 1k was synthesised via General procedure C with sulfide 1ki (2.48 g, $8.85 \mathrm{mmol}), \mathrm{Ph}_{2} \mathrm{IOTf}$ $(3.17 \mathrm{~g}, 7.38 \mathrm{mmol})$ and $\mathrm{Cu}(\mathrm{OTf})_{2}(133 \mathrm{mg}, 0.37 \mathrm{mmol})$. Purification by FCC $\left(10 \% \mathrm{MeOH}\right.$ in $\left.\mathrm{CH}_{2} \mathrm{Cl}_{2}\right)$ gave sulfonium salt $\mathbf{1 k}$ as a brown oil $(2.87 \mathrm{~g}, 77 \%)$.

TLC: $R_{\mathrm{f}}=0.36\left(10 \% \mathrm{MeOH}\right.$ in $\left.\mathrm{CH}_{2} \mathrm{Cl}_{2}\right)$.

${ }^{1} \mathrm{H}$ NMR (500 MHz, Chloroform- $d$ ) $\delta 8.77$ (d, $\left.J=2.1 \mathrm{~Hz}, 1 \mathrm{H}\right), 8.55-8.47$ (m, 1H), $8.25-8.18$ (m, $1 \mathrm{H}), 7.90(\mathrm{~d}, J=7.9 \mathrm{~Hz}, 2 \mathrm{H}), 7.85(\mathrm{~d}, J=8.3 \mathrm{~Hz}, 2 \mathrm{H}), 7.73(\mathrm{t}, J=7.4 \mathrm{~Hz}, 1 \mathrm{H}), 7.65(\mathrm{t}, J=7.5 \mathrm{~Hz}, 2 \mathrm{H})$, $7.47(\mathrm{~d}, J=7.5 \mathrm{~Hz}, 2 \mathrm{H}), 2.45\left(\mathrm{~s}, 3 \mathrm{H}, \mathrm{CH}_{3}\right)$.

${ }^{13} \mathrm{C}$ NMR (126 MHz, Chloroform-d) $\delta 153.4(\mathrm{CH}), 146.7(\mathrm{C}), 145.5(\mathrm{C}), 143.2(\mathrm{CH}), 134.7(\mathrm{CH})$, $132.19(\mathrm{CH}), 132.17(\mathrm{CH}), 131.8(\mathrm{CH}), 131.4(\mathrm{CH}), 131.2(\mathrm{CH}), 126.9(\mathrm{C}), 124.9(\mathrm{C}), 120.9$ (q, $J=$ $320.5 \mathrm{~Hz}, \mathrm{OTf}), 120.6(\mathrm{C}), 21.8\left(\mathrm{CH}_{3}\right)$.

${ }^{19}$ F NMR (470 MHz, Chloroform- $d$ ) $\delta$-78.2.

HRMS (ESI-TOF) $m / z$ : [M-OTf] ${ }^{+}$Calcd for $\mathrm{C}_{18} \mathrm{H}_{15} \mathrm{BrNS}$ : 356.0103, 358.0083; Found: 356.0104, 358.0083 .

(5-Chloropyridin-2-yl)(phenyl)(p-tolyl)sulfonium trifluoromethanesulfonate 11<smiles>Cc1ccc([S+](c2ccccc2)c2ccc(Cl)cn2)cc1</smiles>

Product 11 was synthesised via General procedure $\mathrm{C}$ with sulfide 1 li $(0.32 \mathrm{~g}, 1.35 \mathrm{mmol}), \mathrm{Ph}_{2} \operatorname{IOTf}(0.58$ g, $1.35 \mathrm{mmol})$ and $\mathrm{Cu}(\mathrm{OTf})_{2}(25 \mathrm{mg}, 0.07 \mathrm{mmol})$. Purification by $\mathrm{FCC}\left(10 \% \mathrm{MeOH}\right.$ in $\left.\mathrm{CH}_{2} \mathrm{Cl}_{2}\right)$ gave sulfonium salt 11 as a brown oil $(0.410 \mathrm{~g}, 66 \%)$.

TLC: $R_{\mathrm{f}}=0.30\left(10 \% \mathrm{MeOH}\right.$ in $\left.\mathrm{CH}_{2} \mathrm{Cl}_{2}\right)$.

${ }^{1} \mathrm{H}$ NMR (500 MHz, Chloroform- $d$ ) $\delta 8.67(\mathrm{~d}, J=2.2 \mathrm{~Hz}, 1 \mathrm{H}), 8.37(\mathrm{~d}, J=8.4 \mathrm{~Hz}, 1 \mathrm{H}), 8.05$ (dd, $J=$ 8.3, $2.5 \mathrm{~Hz}, 1 \mathrm{H}), 7.88-7.82(\mathrm{~m}, 2 \mathrm{H}), 7.80(\mathrm{~d}, J=8.4 \mathrm{~Hz}, 2 \mathrm{H}), 7.76-7.70(\mathrm{~m}, 1 \mathrm{H}), 7.69-7.62$ (m, $2 \mathrm{H}), 7.46(\mathrm{~d}, J=8.3 \mathrm{~Hz}, 2 \mathrm{H}), 2.44\left(\mathrm{~s}, 3 \mathrm{H}, \mathrm{CH}_{3}\right)$. 
${ }^{13} \mathrm{C}$ NMR (101 MHz, Chloroform- $d$ ) $\delta 151.3(\mathrm{CH}), 146.8(\mathrm{C}), 144.7(\mathrm{C}), 140.2(\mathrm{CH}), 137.9(\mathrm{C}), 134.7$ $(\mathrm{CH}), 132.2(\mathrm{CH}), 131.9(\mathrm{CH}), 131.6(\mathrm{CH}), 131.4(\mathrm{CH}), 130.6(\mathrm{CH}), 124.8(\mathrm{C}), 120.9(\mathrm{q}, J=320.6 \mathrm{~Hz}$, OTf), $120.4(\mathrm{C}), 21.8\left(\mathrm{CH}_{3}\right)$.

${ }^{19}$ F NMR (376 MHz, Chloroform- $d$ ) $\delta-78.2$.

HRMS (ESI-TOF) $m / z$ : [M-OTf] ${ }^{+}$Calcd for $\mathrm{C}_{18} \mathrm{H}_{15} \mathrm{ClNS}$ : 312.0608, 314.0579; Found: 312.0610, 314.0578 .

\section{Phenyl(pyrimidin-2-yl)(p-tolyl)sulfonium trifluoromethanesulfonate 1m}<smiles>Cc1ccc([S+](c2ccccc2)c2ncccn2)cc1</smiles>

Product $1 \mathrm{~m}$ was synthesised via General procedure $\mathrm{C}$ with sulfide $1 \mathrm{mi}(1.71 \mathrm{~g}, 8.45 \mathrm{mmol}), \mathrm{Ph}_{2} \mathrm{IOTf}$ (3.64 g, $8.45 \mathrm{mmol})$ and $\mathrm{Cu}(\mathrm{OTf})_{2}(153 \mathrm{mg}, 0.42 \mathrm{mmol})$. Purification by $\mathrm{FCC}\left(10 \% \mathrm{MeOH}\right.$ in $\left.\mathrm{CH}_{2} \mathrm{Cl}_{2}\right)$ gave sulfonium salt $1 \mathrm{~m}$ as a brown solid $(2.50 \mathrm{~g}, 69 \%)$.

TLC: $R_{\mathrm{f}}=0.32\left(10 \% \mathrm{MeOH}\right.$ in $\left.\mathrm{CH}_{2} \mathrm{Cl}_{2}\right)$.

${ }^{1} \mathrm{H}$ NMR (500 MHz, Chloroform- $d$ ) $\delta 8.89(\mathrm{~d}, J=4.8 \mathrm{~Hz}, 2 \mathrm{H}), 7.87$ (d, $\left.J=7.6 \mathrm{~Hz}, 2 \mathrm{H}\right), 7.78(\mathrm{~d}, J=8.5$ $\mathrm{Hz}, 2 \mathrm{H}), 7.77-7.75(\mathrm{~m}, 1 \mathrm{H}), 7.72$ (t, $J=7.4 \mathrm{~Hz}, 1 \mathrm{H}), 7.68-7.63(\mathrm{~m}, 2 \mathrm{H}), 7.47$ (d, $J=8.3 \mathrm{~Hz}, 2 \mathrm{H})$, $2.42\left(\mathrm{~s}, 3 \mathrm{H}, \mathrm{CH}_{3}\right)$.

${ }^{13} \mathrm{C}$ NMR (126 MHz, Chloroform-d) $\delta 161.0(\mathrm{CH}), 160.1(\mathrm{C}), 147.0(\mathrm{C}), 135.0(\mathrm{CH}), 132.5(\mathrm{CH}), 132.3$ (CH), $132.2(\mathrm{CH}), 131.5(\mathrm{CH}), 124.9(\mathrm{CH}), 124.4(\mathrm{C}), 120.8$ (q, $J=320.8 \mathrm{~Hz}, \mathrm{OTf}), 120.0(\mathrm{C}), 21.8$ $\left(\mathrm{CH}_{3}\right)$.

${ }^{19} \mathrm{~F}$ NMR (376 MHz, Chloroform- $d$ ) $\delta$-78.2.

HRMS (ESI-TOF) $m / z$ : [M-OTf] ${ }^{+}$Calcd for $\mathrm{C}_{17} \mathrm{H}_{15} \mathrm{~N}_{2} \mathrm{~S}: 279.0950$; Found: 279.0953 . 


\section{Synthesis of bis-heteroaryls}

General procedure D: Halopyridine $(0.45 \mathrm{mmol}, 1.5$ equiv.) was added to an oven-dried crimptop vial and sealed. The vial was evacuated and purged with $\mathrm{N}_{2}$ three times. The halopyridine was dissolved in dry THF $(1.5 \mathrm{~mL})$ and heated/cooled to a given temperature. $i$-PrMgCl$\cdot \mathrm{LiCl}(0.38 \mathrm{~mL}, 0.45 \mathrm{mmol}, 1.5$ equiv., 1.2 M in THF) was added dropwise to the stirring solution over $2 \mathrm{~min}$. The reaction was allowed to stir for a given time at the specified temperature. Sulfonium salt ( $0.3 \mathrm{mmol}, 1.0$ equiv.) was added to a separate oven-dried crimptop vial and dissolved in dry THF $(1.5 \mathrm{~mL})$. The solution of sulfonium salt was added dropwise down the side of the vial to the Grignard reagent solution over $2 \mathrm{~min}$. The reaction was allowed to stir for $2 \mathrm{~h}$. Sat. aq. $\mathrm{NH}_{4} \mathrm{Cl}(3 \mathrm{~mL})$ was added slowly to quench any excess Grignard reagent. The product was extracted with EtOAc $(3 \times 10 \mathrm{~mL})$, the combined organic layers were washed with $\mathrm{H}_{2} \mathrm{O}(20 \mathrm{~mL})$ and brine $(20 \mathrm{~mL})$. The solvent was evaporated, and the product was isolated by FCC.

General procedure E: Pyridine ( $0.45 \mathrm{mmol}, 1.5$ equiv.) was added to an oven-dried crimptop vial and sealed. The vial was evacuated and purged with $\mathrm{N}_{2}$ three times. The pyridine was dissolved in dry THF $(1.5 \mathrm{~mL})$ and heated/cooled to a given temperature. $\mathrm{TMPMgCl} \cdot \mathrm{LiCl}(0.41 \mathrm{~mL}, 0.45 \mathrm{mmol}, 1.5$ equiv., 1.1 $\mathrm{M}$ in THF) was added dropwise to the stirring solution over $2 \mathrm{~min}$. The reaction was allowed to stir for a given time at the specified temperature. Sulfonium salt $(0.3 \mathrm{mmol}, 1.0$ equiv. $)$ was added to a separate oven-dried crimptop vial and dissolved in dry THF $(1.5 \mathrm{~mL})$. The solution of sulfonium salt was added dropwise down the side of the vial to the Grignard reagent solution over $2 \mathrm{~min}$. The reaction was allowed to stir for $2 \mathrm{~h}$. Sat. aq. $\mathrm{NH}_{4} \mathrm{Cl}(3 \mathrm{~mL})$ was added slowly to quench any excess Grignard reagent. The product was extracted with EtOAc $(3 \times 10 \mathrm{~mL})$, the combined organic layers were washed with $\mathrm{H}_{2} \mathrm{O}(20 \mathrm{~mL})$ and brine $(20 \mathrm{~mL})$. The solvent was evaporated, and the product was isolated by FCC.

\section{2,2'-Bipyridine 2}

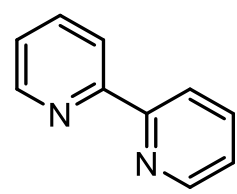

Product 2 was synthesised via General procedure D using 2-iodopyridine (32 $\mu \mathrm{L}, 0.45 \mathrm{mmol})$ and sulfonium salt 1a $(128.2 \mathrm{mg}, 0.3 \mathrm{mmol})$. The Grignard reagent was formed at $0{ }^{\circ} \mathrm{C}$ for $30 \mathrm{~min}$ and the ligand coupling reaction was stirred at $0{ }^{\circ} \mathrm{C}$. Purification by $\mathrm{FCC}\left(80 \% \mathrm{Et}_{2} \mathrm{O}\right.$ in pentane) gave bipyridine 2 as a white solid (24.8 $\mathrm{mg}, 53 \%)$.

${ }^{1} \mathrm{H}$ NMR (400 MHz, Chloroform-d) $\delta 8.74-8.61(\mathrm{~m}, 2 \mathrm{H}), 8.40(\mathrm{~d}, J=8.0 \mathrm{~Hz}, 2 \mathrm{H}), 7.82(\mathrm{td}, J=7.7$, $1.8 \mathrm{~Hz}, 2 \mathrm{H}), 7.31$ (ddd, $J=7.6,4.8,1.2 \mathrm{~Hz}, 2 \mathrm{H}) \mathrm{ppm}$. 
${ }^{13} \mathrm{C}$ NMR (101 MHz, Chloroform- $d$ ) $\delta$ 156.3, 149.4, 137.1, 123.9, $121.2 \mathrm{ppm}$.

Spectra are consistent with literature data. ${ }^{6}$

\section{2,3'-Bipyridine 3}

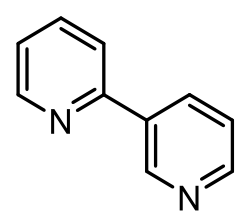

Product 3 was synthesised via General procedure D using 3-iodopyridine (93 mg, $0.45 \mathrm{mmol}$ ) and sulfonium salt 1a $(128.2 \mathrm{mg}, 0.3 \mathrm{mmol})$. The Grignard reagent was formed at $\mathrm{rt}$ for $30 \mathrm{~min}$ and the ligand coupling reaction was stirred at rt. Purification by $\mathrm{FCC}\left(80 \% \mathrm{Et}_{2} \mathrm{O}\right.$ in pentane) gave bipyridine 3 as a yellow oil ( $46.0 \mathrm{mg}, 98 \%)$.

${ }^{1} \mathrm{H}$ NMR $(400 \mathrm{MHz}$, Chloroform- $d) \delta 9.20(\mathrm{~d}, J=2.3 \mathrm{~Hz}, 1 \mathrm{H}), 8.76-8.70(\mathrm{~m}, 1 \mathrm{H}), 8.66(\mathrm{dd}, J=4.9$, $1.7 \mathrm{~Hz}, 1 \mathrm{H}), 8.33$ (dt, $J=8.1,2.0 \mathrm{~Hz}, 1 \mathrm{H}), 7.85-7.72(\mathrm{~m}, 2 \mathrm{H}), 7.41(\mathrm{dd}, J=7.9,4.9 \mathrm{~Hz}, 1 \mathrm{H}), 7.33-$ $7.26(\mathrm{~m}, 1 \mathrm{H}) \mathrm{ppm}$.

${ }^{13} \mathrm{C}$ NMR (101 MHz, Chloroform-d) $\delta 154.9,150.2,150.0,148.3,137.1,135.0,134.5,123.7,123.0$, $120.7 \mathrm{ppm}$.

Spectra are consistent with literature data. ${ }^{7}$

\section{2,4'-Bipyridine 4}<smiles>c1ccc(-c2ccncc2)nc1</smiles>

Product 4 was synthesised via General procedure D using 4-iodopyridine (93 mg, $0.45 \mathrm{mmol}$ ) and sulfonium salt 1a (128.2 mg, $0.3 \mathrm{mmol})$. The Grignard reagent was formed at $\mathrm{rt}$ for $30 \mathrm{~min}$ and the ligand coupling reaction was stirred at $\mathrm{rt}$. Purification by $\mathrm{FCC}\left(70 \% \mathrm{Et}_{2} \mathrm{O}\right.$ in pentane) gave bipyridine 4 as a yellow oil (34.6 $\mathrm{mg}, 74 \%)$.

${ }^{1} \mathrm{H}$ NMR (500 MHz, Chloroform-d) $\delta 8.80-8.61(\mathrm{~m}, 3 \mathrm{H}), 7.95-7.86(\mathrm{~m}, 2 \mathrm{H}), 7.85-7.78(\mathrm{~m}, 2 \mathrm{H})$, 7.34 (ddd, $J=6.4,4.8,2.3 \mathrm{~Hz}, 1 \mathrm{H}) \mathrm{ppm}$.

${ }^{13} \mathrm{C}$ NMR (126 MHz, Chloroform-d) $\delta 154.8,150.6,150.3,146.5,137.2,123.9,121.2,121.0 \mathrm{ppm}$. Spectra are consistent with literature data. ${ }^{8}$ 


\section{4-Methyl-4'-(trifluoromethyl)-2,2'-bipyridine 5}<smiles>Cc1ccnc(-c2cc(C(F)(F)F)ccn2)c1</smiles>

Product 5 was synthesised via General procedure D using 2-bromo-4-methylpyridine (50.1 $\mu \mathrm{L}, 0.45$ mmol) and sulfonium salt 1c (148.8 mg, $0.3 \mathrm{mmol})$. The Grignard reagent was formed at $30{ }^{\circ} \mathrm{C}$ for $2 \mathrm{~h}$ and the ligand coupling reaction was stirred at $\mathrm{rt}$. Purification by $\mathrm{FCC}\left(5 \% \mathrm{Et}_{2} \mathrm{O}\right.$ in pentane) gave bipyridine 5 as a white solid (46.2 $\mathrm{mg}, 64 \%)$.

TLC: $R_{\mathrm{f}}=0.18\left(10 \% \mathrm{Et}_{2} \mathrm{O}\right.$ in pentane $)$.

${ }^{1} \mathrm{H}$ NMR (500 MHz, Chloroform- $d$ ) $\delta 8.84\left(\mathrm{dt}, J=5.0,0.7 \mathrm{~Hz}, 1 \mathrm{H}, \mathrm{NCHCHCCF}_{3}\right), 8.68(\mathrm{dt}, J=1.7$, $\left.0.8 \mathrm{~Hz}, 1 \mathrm{H}, \mathrm{CF}_{3} \mathrm{CCHC}\right), 8.56\left(\mathrm{dd}, J=4.9,0.8 \mathrm{~Hz}, 1 \mathrm{H}, \mathrm{CH}_{3} \mathrm{CCHCH}\right), 8.27$ (dt, $J=1.7,0.8 \mathrm{~Hz}, 1 \mathrm{H}$, $\mathrm{CH}_{3} \mathrm{CCH}$ ), 7.51 (ddd, $J=5.0,1.8,0.8 \mathrm{~Hz}, 1 \mathrm{H}, \mathrm{NCHCHCCF}_{3}$ ), 7.19 (ddd, $J=5.0,1.7,0.8 \mathrm{~Hz}, 1 \mathrm{H}$, $\left.\mathrm{CH}_{3} \mathrm{CCHCH}\right), 2.46\left(\mathrm{~s}, 3 \mathrm{H}, \mathrm{CH}_{3}\right) \mathrm{ppm}$.

${ }^{13} \mathrm{C}$ NMR (126 MHz, Chloroform- $d$ ) $\delta 157.9$ (C), 154.3 (C), $150.1 \quad\left(\mathrm{NCHCHCCF}_{3}\right), 149.3$ $\left(\mathrm{CH}_{3} \mathrm{CCHCH}\right), 148.6\left(\mathrm{CH}_{3} \mathrm{C}\right), 139.5$ (q, $\left.J=34.2 \mathrm{~Hz}, \mathrm{CF}_{3} \mathrm{C}\right), 125.6\left(\mathrm{CH}_{3} \mathrm{CCHCH}\right), 123.1$ (q, $J=273.2$ $\left.\mathrm{Hz}, \mathrm{CF}_{3}\right), 122.3\left(\mathrm{CH}_{3} \mathrm{CCH}\right), 119.2\left(\mathrm{q}, J=3.5 \mathrm{~Hz}, \mathrm{CF}_{3} \mathrm{CCHCH}\right), 117.2\left(\mathrm{q}, J=3.6 \mathrm{~Hz}, \mathrm{CF}_{3} \mathrm{CCHC}\right), 21.4$ ppm.

${ }^{19} \mathrm{~F}$ NMR $\left(470 \mathrm{MHz}, \mathrm{CDCl}_{3}\right) \delta-64.8 \mathrm{ppm}$.

Spectra are consistent with literature data. ${ }^{9}$

\section{4-Fluoro-4'-(trifluoromethyl)-2,2'-bipyridine 6}<smiles>Fc1ccnc(-c2cc(C(F)(F)F)ccn2)c1</smiles>

Product 6 was synthesised via General procedure D using 2-bromo-4-fluoropyridine (46.6 $\mu \mathrm{L}, 0.45$ mmol) and sulfonium salt 1c (148.8 mg, $0.3 \mathrm{mmol})$. The Grignard reagent was formed at $\mathrm{rt}$ for $1 \mathrm{~h}$ and the ligand coupling reaction was stirred at rt. Purification by $\mathrm{FCC}\left(7.5 \% \mathrm{Et}_{2} \mathrm{O}\right.$ in pentane) gave bipyridine 6 as a yellow oil (52.8 $\mathrm{mg}, 73 \%)$.

TLC: $R_{\mathrm{f}}=0.55\left(10 \% \mathrm{Et}_{2} \mathrm{O}\right.$ in pentane $)$. 
${ }^{1} \mathrm{H}$ NMR $\left(500 \mathrm{MHz}\right.$, Chloroform- $d$ ) $\delta 8.84\left(\mathrm{dt}, J=5.0,0.7 \mathrm{~Hz}, 1 \mathrm{H}, \mathrm{NCHCHCCF}_{3}\right), 8.69(\mathrm{dt}, J=1.7$, $\left.0.8 \mathrm{~Hz}, 1 \mathrm{H}, \mathrm{F}_{3} \mathrm{CCCH}\right), 8.66$ (dd, $\left.J=8.4,5.5 \mathrm{~Hz}, 1 \mathrm{H}, \mathrm{NCHCHCF}\right), 8.20$ (dd, $J=10.2,2.5 \mathrm{~Hz}, 1 \mathrm{H}$, FCCHC), $7.59-7.51\left(\mathrm{~m}, 1 \mathrm{H}, \mathrm{NCHCHCCF}_{3}\right), 7.10$ (ddd, $\left.J=8.1,5.5,2.5 \mathrm{~Hz}, 1 \mathrm{H}, \mathrm{FCCHCH}\right) \mathrm{ppm}$.

${ }^{13} \mathrm{C}$ NMR (126 MHz, Chloroform- $d$ ) $\delta 169.7$ (d, $\left.J=262.3 \mathrm{~Hz}, \mathrm{CF}\right), 158.2(\mathrm{~d}, J=7.4 \mathrm{~Hz}, \mathrm{FCCHC})$, $156.5\left(\mathrm{~d}, J=3.8 \mathrm{~Hz}, \mathrm{NCCHCCF}_{3}\right), 151.8(\mathrm{~d}, J=6.9 \mathrm{~Hz}, \mathrm{FCCHCHN}), 150.3\left(\mathrm{NCHCHCCF}_{3}\right), 139.6(\mathrm{q}$, $J=34.2 \mathrm{~Hz}, \mathrm{CF}_{3} C$ ), 123.0 (q, $J=273.4 \mathrm{~Hz}, \mathrm{CF}_{3}$ ), 119.8 (q, $\left.J=3.4 \mathrm{~Hz}, \mathrm{CF}_{3} \mathrm{CCH}\right), 117.3$ (q, $J=3.7 \mathrm{~Hz}$, $\left.\mathrm{CF}_{3} \mathrm{CCH}\right), 112.4(\mathrm{~d}, J=16.8 \mathrm{~Hz}, \mathrm{FCCHC}), 109.3(\mathrm{~d}, J=18.7 \mathrm{~Hz}, \mathrm{FCCHCH}) \mathrm{ppm}$.

${ }^{19}$ F NMR (470 MHz, Chloroform- $d$ ) $\delta-64.9\left(\mathrm{CF}_{3}\right),-101.3$ (app. dt, $J=10.3,8.2 \mathrm{~Hz}, \mathrm{CF}$ ) ppm. HRMS (ESI-TOF) m/z: [M+H] ${ }^{+}$Calcd for $\mathrm{C}_{11} \mathrm{H}_{7} \mathrm{~F}_{4} \mathrm{~N}_{2}: 243.0540$; found: $243.0539 \mathrm{ppm}$.

1-(5-Methoxypyridin-2-yl)isoquinoline 7

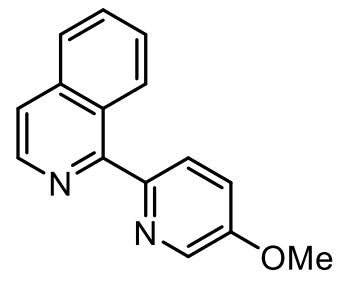

Product 7 was synthesised via General procedure D using 2-bromo-5-methoxypyridine (56 $\mu \mathrm{L}, 0.45$ mmol) and sulfonium salt $\mathbf{1 b}(143.3 \mathrm{mg}, 0.3 \mathrm{mmol})$. The Grignard reagent was formed at $\mathrm{rt}$ for $3 \mathrm{~h}$ and the ligand coupling reaction was stirred at rt. Purification by $\mathrm{FCC}\left(50 \% \mathrm{Et}_{2} \mathrm{O}\right.$ in pentane) gave bipyridine 7 as a white solid (55.3 $\mathrm{mg}, 78 \%$ ).

TLC: $R_{\mathrm{f}}=0.1\left(50 \% \mathrm{Et}_{2} \mathrm{O}\right.$ in pentane).

${ }^{1} \mathrm{H}$ NMR (500 MHz, Chloroform- $d$ ) $\delta 8.67$ (dd, $\left.J=8.6,1.0 \mathrm{~Hz}, 1 \mathrm{H}, \mathrm{NCCCH}\right), 8.60(\mathrm{~d}, J=5.6 \mathrm{~Hz}, 1 \mathrm{H}$, $\mathrm{NCH}), 8.49$ (d, $\left.J=2.9 \mathrm{~Hz}, 1 \mathrm{H}, \mathrm{NCHCOCH}_{3}\right), 7.99$ (dd, $\left.J=8.6,0.7 \mathrm{~Hz}, 1 \mathrm{H}, \mathrm{CH}_{3} \mathrm{OCCHCH}\right), 7.88-$ $7.83(\mathrm{~m}, 1 \mathrm{H}, \mathrm{NCCCHCH}), 7.72-7.65(\mathrm{~m}, 2 \mathrm{H}, \mathrm{ArH}), 7.59$ (ddd, $J=8.3,6.8,1.3 \mathrm{~Hz}, 1 \mathrm{H}$, $\mathrm{NCCCHCHCH}$ ), 7.41 (dd, $J=8.6,3.0 \mathrm{~Hz}, 1 \mathrm{H}, \mathrm{CH}_{3} \mathrm{OCCH}$ ), 3.97 (s, $\left.3 \mathrm{H} . \mathrm{OCH}_{3}\right) \mathrm{ppm}$.

${ }^{13} \mathrm{C}$ NMR (126 MHz, Chloroform- $d$ ) $\delta 157.4(\mathrm{NCC}), 155.7\left(\mathrm{COCH}_{3}\right), 151.1(\mathrm{C}), 142.0(\mathrm{NCH}), 137.4$ (C), $136.4(\mathrm{NCHCO}), 130.1(\mathrm{CH}), 128.1(\mathrm{NCHCH}), 127.6(\mathrm{NCCCHCHCH}), 127.0(\mathrm{NCCCHCH})$, $126.8(\mathrm{C}), 125.9\left(\mathrm{CH}_{3} \mathrm{OCCHCH}\right), 121.4\left(\mathrm{CH}_{3} \mathrm{OCCH}\right), 120.9(\mathrm{CH}), 55.9\left(\mathrm{OCH}_{3}\right) \mathrm{ppm}$.

HRMS (ESI-TOF) $m / z$ : [M+H] $]^{+}$Calcd for $\mathrm{C}_{15} \mathrm{H}_{13} \mathrm{~N}_{2} \mathrm{O}: 237.1022$; found: 237.1023. 


\section{5'-Bromo-2,3'-bipyridine 8}<smiles>Brc1cncc(-c2ccccn2)c1</smiles>

Product 8 was synthesised via General procedure D using 3,5-dibromopyridine (106.6 mg, $0.45 \mathrm{mmol}$ ) and sulfonium salt 1a $(128.2 \mathrm{mg}, 0.3 \mathrm{mmol})$. The Grignard reagent was formed at $\mathrm{rt}$ for $1 \mathrm{~h}$ and the ligand coupling reaction was stirred at rt. Purification by $\mathrm{FCC}\left(40 \% \mathrm{Et}_{2} \mathrm{O}\right.$ in pentane) gave bipyridine 8 as a yellow solid (43.7 mg, 62\%).

TLC: $R_{\mathrm{f}}=0.22\left(40 \% \mathrm{Et}_{2} \mathrm{O}\right.$ in pentane)

${ }^{1} \mathrm{H}$ NMR $(500 \mathrm{MHz}$, Chloroform- $d$ ) $\delta 9.09$ (d, $J=1.9 \mathrm{~Hz}, 1 \mathrm{H}), 8.72(\mathrm{dt}, J=4.8,1.3 \mathrm{~Hz}, 1 \mathrm{H}), 8.70$ (d, $J$ $=2.2 \mathrm{~Hz}, 1 \mathrm{H}), 8.51(\mathrm{t}, J=2.0 \mathrm{~Hz}, 1 \mathrm{H}), 7.81(\mathrm{dd}, J=7.7,1.8 \mathrm{~Hz}, 1 \mathrm{H}), 7.74(\mathrm{~d}, J=7.7 \mathrm{~Hz}, 1 \mathrm{H}), 7.32$ (ddd, $J=7.3,4.7,0.8 \mathrm{~Hz}, 1 \mathrm{H}$ ).

${ }^{13} \mathrm{C}$ NMR (126 MHz, Chloroform- $d$ ) $\delta 153.4,151.0,150.4,146.3,137.3,137.1,136.5,123.5,121.3$, 120.9 .

Spectra were consistent with literature data. ${ }^{10}$

\section{6-Bromo-2,3'-bipyridine 9}<smiles>Brc1cccc(-c2cccnc2)n1</smiles>

Product 9 was synthesised via General procedure D using 3-iodopyridine $(92.3 \mathrm{mg}, 0.45 \mathrm{mmol})$ and sulfonium salt $1 \mathrm{~g}(151.9 \mathrm{mg}, 0.3 \mathrm{mmol})$. The Grignard reagent was formed at $\mathrm{rt}$ for $45 \mathrm{~min}$ and the ligand coupling reaction was stirred at $\mathrm{rt}$. Purification by $\mathrm{FCC}\left(80 \% \mathrm{Et}_{2} \mathrm{O}\right.$ in pentane) gave bipyridine 9 as a yellow solid (46.8 $\mathrm{mg}, 66 \%)$.

TLC: $R_{\mathrm{f}}=0.19\left(80 \% \mathrm{Et}_{2} \mathrm{O}\right.$ in pentane $)$

${ }^{1} \mathrm{H}$ NMR (500 MHz, Chloroform- $d$ ) $\delta 9.15(\mathrm{~d}, J=2.3 \mathrm{~Hz}, 1 \mathrm{H}), 8.65(\mathrm{dd}, J=4.9,1.6 \mathrm{~Hz}, 1 \mathrm{H}), 8.32$ (dt, $J=7.9,2.1 \mathrm{~Hz}, 1 \mathrm{H}), 7.70(\mathrm{~d}, J=7.7 \mathrm{~Hz}, 1 \mathrm{H}), 7.63(\mathrm{t}, J=7.8 \mathrm{~Hz}, 1 \mathrm{H}), 7.46(\mathrm{~d}, J=7.7 \mathrm{~Hz}, 1 \mathrm{H}), 7.39$ $(\mathrm{dd}, J=8.0,4.8 \mathrm{~Hz}, 1 \mathrm{H})$.

${ }^{13} \mathrm{C}$ NMR (126 MHz, Chloroform- $d$ ) $\delta 156.0,150.6,148.2,142.7,139.3,134.6,133.4,127.3,123.8$, 119.2 .

Spectra were consistent with literature data. ${ }^{11}$ 


\section{6'-Bromo-6-(trifluoromethyl)-2,3'-bipyridine 10}<smiles>FC(F)(F)c1cccc(-c2ccc(Br)nc2)n1</smiles>

Product 10 was synthesised via General procedure D using 2,5-dibromopyridine (106.6 mg, $0.45 \mathrm{mmol}$ ) and sulfonium salt $1 \mathbf{i}(148.6 \mathrm{mg}, 0.3 \mathrm{mmol})$. The Grignard reagent was formed at $\mathrm{rt}$ for $1 \mathrm{~h}$ and the ligand coupling reaction was stirred at rt. Purification by $\mathrm{FCC}\left(20 \% \mathrm{Et}_{2} \mathrm{O}\right.$ in pentane) gave bipyridine 10 as a yellow solid (45.0 mg, 50\%).

TLC: $R_{\mathrm{f}}=0.19\left(20 \% \mathrm{Et}_{2} \mathrm{O}\right.$ in pentane $)$

${ }^{1} \mathrm{H}$ NMR (400 MHz, Chloroform- $d$ ) $\delta 8.96(\mathrm{~d}, J=2.5 \mathrm{~Hz}, 1 \mathrm{H}, \mathrm{CHN}), 8.29(\mathrm{dd}, J=8.3,2.7 \mathrm{~Hz}, 1 \mathrm{H})$, $7.99(\mathrm{t}, J=7.8 \mathrm{~Hz}, 1 \mathrm{H}), 7.93(\mathrm{~d}, J=7.6 \mathrm{~Hz}, 1 \mathrm{H}), 7.69(\mathrm{dd}, J=7.5,1.1 \mathrm{~Hz}, 1 \mathrm{H}), 7.61(\mathrm{dd}, J=8.3,0.5$ $\mathrm{Hz}, 1 \mathrm{H}) .{ }^{13} \mathrm{C}$ NMR (101 MHz, Chloroform- $d$ ) $\delta 154.2(\mathrm{C}), 148.9$ (q, $J=35.0 \mathrm{~Hz}, C \mathrm{CF}_{3}$ ), $148.6(\mathrm{CH})$, $143.7(\mathrm{CBr}), 138.8(\mathrm{CH}), 137.3(\mathrm{CH}), 132.8(\mathrm{C}), 128.5(\mathrm{CH}), 122.8(\mathrm{CH}), 121.4\left(\mathrm{q}, J=276.6 \mathrm{~Hz}, \mathrm{CF}_{3}\right)$, $119.8(\mathrm{q}, J=2.7 \mathrm{~Hz}, \mathrm{CH})$.

${ }^{19} \mathrm{~F}$ NMR (282 MHz, Chloroform- $\left.d\right) \delta-68.2$.

HRMS (ESI-TOF) $m / z$ : $[\mathrm{M}+\mathrm{H}]^{+}$Calcd for $\mathrm{C}_{11} \mathrm{H}_{7} \mathrm{BrF}_{3} \mathrm{~N}_{2}$ : 302.9739, 304.9719; Found: 302.9741, 304.9721.

\section{5-Chloro-2,3'-bipyridine 11}<smiles>Clc1ccc(-c2cccnc2)nc1</smiles>

Product 11 was synthesised via General procedure D using 3-iodopyridine ( $92.3 \mathrm{mg}, 0.45 \mathrm{mmol})$ and sulfonium salt 11 (138.6 mg, $0.3 \mathrm{mmol})$. The Grignard reagent was formed at $\mathrm{rt}$ for $45 \mathrm{~min}$ and the ligand coupling reaction was stirred at rt. Purification by $\mathrm{FCC}\left(80 \% \mathrm{Et}_{2} \mathrm{O}\right.$ in pentane) gave bipyridine 11 as a white solid (31.9 mg, 56\%).

TLC: $R_{\mathrm{f}}=0.27\left(80 \% \mathrm{Et}_{2} \mathrm{O}\right.$ in pentane $)$

${ }^{1} \mathrm{H}$ NMR (400 MHz, Chloroform- $d$ ) $\delta 9.17-9.14(\mathrm{~m}, 1 \mathrm{H}), 8.67-8.64(\mathrm{~m}, 2 \mathrm{H}), 8.28(\mathrm{dt}, J=7.9,2.0$ $\mathrm{Hz}, 1 \mathrm{H}), 7.76(\mathrm{dd}, J=8.4,2.5 \mathrm{~Hz}, 1 \mathrm{H}), 7.70(\mathrm{dd}, J=8.6,0.6 \mathrm{~Hz}, 1 \mathrm{H}), 7.40$ (ddd, $J=7.8,4.7,0.6 \mathrm{~Hz}$, $1 \mathrm{H})$. 
${ }^{13} \mathrm{C}$ NMR (101 MHz, Chloroform-d) $\delta 153.0,150.3,149.1,148.2,136.8,134.3,133.9,131.6,123.8$, 121.2 .

Spectra were consistent with literature data. ${ }^{12}$

5,5'-Dibromo-2,3'-bipyridine 12<smiles>Brc1ccc(-c2cncc(Br)c2)nc1</smiles>

Product 12 was synthesised via General procedure D using 3,5-dibromopyridine (106.6 mg, $0.45 \mathrm{mmol}$ ) and sulfonium salt $1 \mathbf{k}(151.9 \mathrm{mg}, 0.3 \mathrm{mmol})$. The Grignard reagent was formed at $\mathrm{rt}$ for $1 \mathrm{~h}$ and the ligand coupling reaction was stirred at $45^{\circ} \mathrm{C}$ for $3.5 \mathrm{~h}$. Purification by $\mathrm{FCC}\left(40 \% \mathrm{Et}_{2} \mathrm{O}\right.$ in pentane) gave bipyridine 12 as a white solid $(63.5 \mathrm{mg}, 67 \%)$.

TLC: $R_{\mathrm{f}}=0.23\left(40 \% \mathrm{Et}_{2} \mathrm{O}\right.$ in pentane $)$

${ }^{1} \mathrm{H}$ NMR (400 MHz, Chloroform- $d$ ) $\delta 9.07$ (d, $\left.J=1.9 \mathrm{~Hz}, 1 \mathrm{H}\right), 8.77$ (d, $\left.J=2.3 \mathrm{~Hz}, 1 \mathrm{H}\right), 8.72(\mathrm{~d}, J=2.3$ $\mathrm{Hz}, 1 \mathrm{H}), 8.49$ (t, $J=2.1 \mathrm{~Hz}, 1 \mathrm{H}), 7.93(\mathrm{dd}, J=8.5,2.4 \mathrm{~Hz}, 1 \mathrm{H}), 7.65(\mathrm{~d}, J=8.3 \mathrm{~Hz}, 1 \mathrm{H})$.

${ }^{13} \mathrm{C}$ NMR (101 MHz, Chloroform- $d$ ) $\delta 151.8(\mathrm{C}), 151.5(\mathrm{CH}), 151.3(\mathrm{CH}), 146.0(\mathrm{CH}), 139.9(\mathrm{CH})$, $136.9(\mathrm{CH}), 135.4(\mathrm{C}), 121.8(\mathrm{CH}), 121.4(\mathrm{C}), 121.0(\mathrm{C})$.

HRMS (ESI-TOF) $m / z$ : $[\mathrm{M}+\mathrm{H}]^{+}$Calcd for $\mathrm{C}_{10} \mathrm{H}_{7} \mathrm{Br}_{2} \mathrm{~N}_{2}$ : 312.8970, 314.8950, 316.8931; Found: $312.8971,314.8952,316.8930$.

\section{[2,3'-Bipyridine]-5-carbonitrile 13}<smiles>N#Cc1ccc(-c2cccnc2)nc1</smiles>

Product 13 was synthesised via General procedure D using 3-iodopyridine ( $92.3 \mathrm{mg}, 0.45 \mathrm{mmol})$ and sulfonium salt $\mathbf{1 j}$ ( $135.7 \mathrm{mg}, 0.3 \mathrm{mmol}$ ). The Grignard reagent was formed at $\mathrm{rt}$ for $45 \mathrm{~min}$ and the ligand coupling reaction was stirred at rt. Purification by $\mathrm{FCC}\left(80 \% \mathrm{Et}_{2} \mathrm{O}\right.$ in pentane) gave bipyridine 13 as a yellow solid (28.4 mg, 52\%).

TLC: $R_{\mathrm{f}}=0.14\left(80 \% \mathrm{Et}_{2} \mathrm{O}\right.$ in pentane $)$

${ }^{1} \mathrm{H}$ NMR (500 MHz, Chloroform- $d$ ) $\delta 9.25(\mathrm{~s}, 1 \mathrm{H}), 8.97$ (d, $\left.J=2.0 \mathrm{~Hz}, 1 \mathrm{H}\right), 8.73(\mathrm{~d}, J=4.8 \mathrm{~Hz}, 1 \mathrm{H})$, $8.37(\mathrm{dt}, J=7.9,1.9 \mathrm{~Hz}, 1 \mathrm{H}), 8.06(\mathrm{dd}, J=8.3,2.0 \mathrm{~Hz}, 1 \mathrm{H}), 7.89(\mathrm{~d}, J=8.4 \mathrm{~Hz}, 1 \mathrm{H}), 7.46(\mathrm{dd}, J=8.0$, $4.8 \mathrm{~Hz}, 1 \mathrm{H})$. 
${ }^{13} \mathrm{C}$ NMR (126 MHz, Chloroform-d) $\delta 158.1(\mathrm{C}), 152.8(\mathrm{CH}), 151.5(\mathrm{CH}), 148.7(\mathrm{CH}), 140.3(\mathrm{CH})$, $134.9(\mathrm{CH}), 133.1(\mathrm{C}), 124.0(\mathrm{CH}), 120.2(\mathrm{CH}), 116.7(\mathrm{CN}), 108.9(\mathrm{CCN})$.

HRMS (ESI-TOF) $m / z$ : $[\mathrm{M}+\mathrm{H}]^{+}$Calcd for $\mathrm{C}_{11} \mathrm{H}_{8} \mathrm{~N}_{3}$ : 182.0713; Found: 182.0714 .

\section{5-Methoxy-2,3'-bipyridine 14}

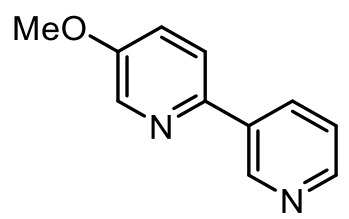

Product 14 was synthesised via General procedure D using 3-iodopyridine ( $92.3 \mathrm{mg}, 0.45 \mathrm{mmol}$ ) and sulfonium salt 1 f $(137.2 \mathrm{mg}, 0.3 \mathrm{mmol})$. The Grignard reagent was formed at $\mathrm{rt}$ for $45 \mathrm{~min}$ and the ligand coupling reaction was stirred at rt. Purification by $\mathrm{FCC}\left(80 \% \mathrm{Et}_{2} \mathrm{O}\right.$ in pentane) gave bipyridine 14 as a white solid (23.1 mg, $41 \%)$.

TLC: $R_{\mathrm{f}}=0.10\left(80 \% \mathrm{Et}_{2} \mathrm{O}\right.$ in pentane $)$

${ }^{1} \mathrm{H}$ NMR (500 MHz, Chloroform- $d$ ) $\delta 9.12(\mathrm{~d}, J=2.3 \mathrm{~Hz}, 1 \mathrm{H}), 8.59$ (dd, $\left.J=4.8,1.6 \mathrm{~Hz}, 1 \mathrm{H}\right), 8.41$ (d, $J=2.9 \mathrm{~Hz}, 1 \mathrm{H}), 8.25(\mathrm{dt}, J=8.1,2.1 \mathrm{~Hz}, 1 \mathrm{H}), 7.68(\mathrm{~d}, J=8.5 \mathrm{~Hz}, 1 \mathrm{H}), 7.36(\mathrm{dd}, J=7.9,4.7 \mathrm{~Hz}, 1 \mathrm{H})$, $7.29(\mathrm{dd}, J=8.7,3.0 \mathrm{~Hz}, 1 \mathrm{H}), 3.90(\mathrm{~s}, 3 \mathrm{H}, \mathrm{OMe})$.

${ }^{13} \mathrm{C}$ NMR (126 MHz, Chloroform- $d$ ) $\delta 155.5(\mathrm{C}), 149.3(\mathrm{CH}), 147.9(\mathrm{CH}), 147.4(\mathrm{C}), 137.9(\mathrm{CH}), 134.7$ (C), $133.9(\mathrm{CH}), 123.7(\mathrm{CH}), 121.3(\mathrm{CH}), 121.0(\mathrm{CH}), 55.8(\mathrm{OMe})$.

HRMS (ESI-TOF) $m / z$ : [M+H] $]^{+}$Calcd for $\mathrm{C}_{11} \mathrm{H}_{11} \mathrm{~N}_{2} \mathrm{O}$ : 187.0866; Found: 187.0867.

\section{2'-Fluoro-2,4'-bipyridine 15}

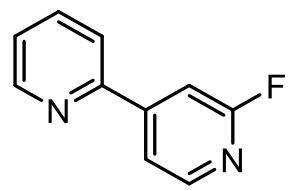

Product 15 was synthesised via General procedure D using 4-bromo-2-fluoropyridine (46.2 $\mu \mathrm{L}, 0.45$ $\mathrm{mmol})$ and sulfonium salt $1 \mathbf{a}(128.2 \mathrm{mg}, 0.3 \mathrm{mmol})$. The Grignard reagent was formed at $\mathrm{rt}$ for $30 \mathrm{~min}$ and the ligand coupling reaction was stirred at rt. Purification by $\mathrm{FCC}\left(70 \% \mathrm{Et}_{2} \mathrm{O}\right.$ in pentane) gave bipyridine 15 as a yellow oil (46.0 mg, $88 \%$ ).

${ }^{1} \mathrm{H}$ NMR (500 MHz, Chloroform- $d$ ) $\delta 8.74$ (ddd, $\left.J=4.8,1.8,1.0 \mathrm{~Hz}, 1 \mathrm{H}\right), 8.31(\mathrm{dt}, J=5.3,0.7 \mathrm{~Hz}, 1 \mathrm{H})$, $7.83(\mathrm{td}, J=7.7,1.8 \mathrm{~Hz}, 1 \mathrm{H}), 7.80-7.75(\mathrm{~m}, 2 \mathrm{H}), 7.62-7.52(\mathrm{~m}, 1 \mathrm{H}), 7.37$ (ddd, $J=7.4,4.8,1.3 \mathrm{~Hz}$, 1H) $\mathrm{ppm}$. 
${ }^{13} \mathrm{C}$ NMR (126 MHz, Chloroform- $d$ ) $\delta 164.9(\mathrm{~d}, J=237.9 \mathrm{~Hz}, \mathrm{CF}), 153.5$ (d, $\left.J=3.8 \mathrm{~Hz}\right), 152.2(\mathrm{~d}, J=$ $8.1 \mathrm{~Hz}), 150.3,148.3$ (d, $J=15.2 \mathrm{~Hz}), 137.3,124.5,121.1,119.0$ (d, $J=4.2 \mathrm{~Hz}), 107.1(\mathrm{~d}, J=39.1 \mathrm{~Hz})$ ppm.

${ }^{19} \mathrm{~F}$ NMR (470 MHz, Chloroform- $d$ ) $\delta-67.8 \mathrm{ppm}$.

Spectra are consistent with literature data. ${ }^{10}$

\section{2'-Fluoro-6-methyl-2,4'-bipyridine 16}<smiles>Cc1cccc(-c2ccnc(F)c2)n1</smiles>

Product 16 was synthesised via General procedure D using 4-bromo-2-fluoropyridine (46.2 $\mu \mathrm{L}, 0.45$ $\mathrm{mmol})$ and sulfonium salt $\mathbf{1 h}(132.4 \mathrm{mg}, 0.3 \mathrm{mmol})$. The Grignard reagent was formed at $0{ }^{\circ} \mathrm{C}$ for 30 min and the ligand coupling reaction was stirred at $0{ }^{\circ} \mathrm{C}$. Purification by $\mathrm{FCC}\left(20 \% \mathrm{Et}_{2} \mathrm{O}\right.$ in pentane) gave bipyridine 16 as a white solid (38.7 $\mathrm{mg}, 69 \%)$.

${ }^{1} \mathrm{H}$ NMR (500 MHz, Chloroform- $d$ ) $\delta 8.30(\mathrm{~d}, J=5.2 \mathrm{~Hz}, 1 \mathrm{H}), 7.77(\mathrm{dt}, J=5.3,1.7 \mathrm{~Hz}, 1 \mathrm{H}), 7.71(\mathrm{t}, J$ $=7.7 \mathrm{~Hz}, 1 \mathrm{H}), 7.61-7.55(\mathrm{~m}, 2 \mathrm{H}), 7.23(\mathrm{~d}, J=7.6 \mathrm{~Hz}, 1 \mathrm{H}), 2.64(\mathrm{~s}, 3 \mathrm{H}) \mathrm{ppm}$.

${ }^{13} \mathrm{C}$ NMR (126 MHz, Chloroform- $d$ ) $\delta 164.9$ (d, $\left.J=237.9 \mathrm{~Hz}, \mathrm{CF}\right), 159.3,152.9$ (d, $\left.J=3.8 \mathrm{~Hz}\right), 152.6$ (d, $J=8.2 \mathrm{~Hz}), 148.2(\mathrm{~d}, J=15.2 \mathrm{~Hz}), 137.4,124.1,119.1$ (d, $J=4.2 \mathrm{~Hz}), 118.2,107.2$ (d, $J=38.9$ $\mathrm{Hz}), 24.8 \mathrm{ppm}$.

${ }^{19} \mathrm{~F}$ NMR (376 MHz, Chloroform- $d$ ) $\delta-68.1 \mathrm{ppm}$.

Spectra are consistent with literature data. ${ }^{10}$

\section{1-(2-Fluoropyridin-4-yl)isoquinoline 17}<smiles>Fc1cc(-c2nccc3ccccc23)ccn1</smiles>

Product 17 was synthesised via General procedure D using 4-bromo-2-fluoropyridine (46.2 $\mu \mathrm{L}, 0.45$ $\mathrm{mmol})$ and sulfonium salt $\mathbf{1 b}(143.3 \mathrm{mg}, 0.3 \mathrm{mmol})$. The Grignard reagent was formed at $0{ }^{\circ} \mathrm{C}$ for 30 min and the ligand coupling reaction was stirred at $0{ }^{\circ} \mathrm{C}$. Purification by $\mathrm{FCC}\left(50 \% \mathrm{Et}_{2} \mathrm{O}\right.$ in pentane) gave bipyridine 17 as a white solid (67.2 $\mathrm{mg}$, quant. yield). 
The reaction was scaled up and product 17 was synthesized via General procedure D using 4-bromo-2fluoropyridine $(1.13 \mathrm{~mL}, 11.0 \mathrm{mmol})$ and sulfonium salt $\mathbf{1 b}(3.50 \mathrm{~g}, 7.3 \mathrm{mmol})$. Purification by FCC (50\% Et2O in pentane) gave bipyridine $\mathbf{1 7}$ as a white solid (1.57 g, 96\%).

TLC: $R_{\mathrm{f}}=0.33\left(35 \% \mathrm{Et}_{2} \mathrm{O}\right.$ in pentane $)$.

${ }^{1} \mathrm{H}$ NMR (500 MHz, Chloroform- $d$ ) $\delta 8.64(\mathrm{~d}, J=5.6 \mathrm{~Hz}, 1 \mathrm{H}, \mathrm{NCH}), 8.40(\mathrm{dt}, J=5.1,0.8 \mathrm{~Hz}, 1 \mathrm{H}$, FCNCH), $8.02(\mathrm{dt}, J=8.6,1.0 \mathrm{~Hz}, 1 \mathrm{H}, \mathrm{NCHCHCCH}), 7.97-7.92(\mathrm{~m}, 1 \mathrm{H}, \mathrm{ArH}), 7.79-7.72(\mathrm{~m}, 2 \mathrm{H}$, ArH), 7.62 (ddd, $J=8.2,6.8,1.2 \mathrm{~Hz}, 1 \mathrm{H}, \mathrm{NCCCHCH}), 7.53$ (dd, $J=5.1,2.0 \mathrm{~Hz}, 1 \mathrm{H}, \mathrm{NCCCHCHN}$ ), $7.27(\mathrm{~d}, J=1.9 \mathrm{~Hz}, \mathrm{FCCH}) \mathrm{ppm}$.

${ }^{13} \mathrm{C}$ NMR (126 MHz, Chloroform- $d$ ) $\delta 163.9(\mathrm{~d}, J=239.4 \mathrm{~Hz}, \mathrm{CF}), 156.7$ (d, $\left.J=3.3 \mathrm{~Hz}, \mathrm{FCCHCC}\right)$, 152.8 (d, $J=8.1 \mathrm{~Hz}, \mathrm{FCCHC}), 147.9$ (d, $J=15.1 \mathrm{~Hz}, \mathrm{FCNCH}), 142.5(\mathrm{NCH}), 136.9(\mathrm{C}), 130.6(\mathrm{CH})$, $128.1(\mathrm{NCCCHCH}), 127.4(\mathrm{CH}), 126.11(\mathrm{NCCCH}), 126.10(\mathrm{C}), 122.5(\mathrm{~d}, J=4.3 \mathrm{~Hz}, \mathrm{FCNCHCH})$, $121.5(\mathrm{CH}), 110.7(\mathrm{~d}, J=38.1 \mathrm{~Hz}, \mathrm{FCCH}) \mathrm{ppm}$.

${ }^{19}$ F NMR (470 MHz, Chloroform- $d$ ) $\delta-67.4$ ppm.

HRMS (ESI-TOF) $m / z$ : [M+H $]^{+}$Calcd for $\mathrm{C}_{14} \mathrm{H}_{10} \mathrm{FN}_{2}: 225.0823$; found: 225.0820 .

\section{1-(2-Methoxypyridin-4-yl)isoquinoline 18}

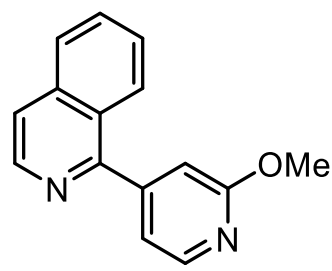

Product 18 was synthesised via General procedure D using 4-bromo-2-methoxypyridine ( $84.6 \mathrm{mg}, 0.45$ mmol) and sulfonium salt $\mathbf{1 b}(143.3 \mathrm{mg}, 0.3 \mathrm{mmol})$. The Grignard reagent was formed at $40{ }^{\circ} \mathrm{C}$ for $2 \mathrm{~h}$ and the ligand coupling reaction was stirred at $\mathrm{rt}$. Purification by FCC (25\% $\mathrm{Et}_{2} \mathrm{O}$ in pentane) gave bipyridine 18 as a white solid (44.7 mg, 63\%).

TLC: $R_{\mathrm{f}}=0.11\left(20 \% \mathrm{Et}_{2} \mathrm{O}\right.$ in pentane $)$.

${ }^{1} \mathrm{H}$ NMR (500 MHz, Chloroform- $d$ ) $\delta 8.61(\mathrm{~d}, J=5.7 \mathrm{~Hz}, 1 \mathrm{H}, \mathrm{NCH}), 8.33$ (dd, $J=5.2,0.8 \mathrm{~Hz}, 1 \mathrm{H}$, $\left.\mathrm{CH}_{3} \mathrm{OCNCH}\right), 8.05$ (dd, $\left.J=8.6,1.0 \mathrm{~Hz}, 1 \mathrm{H}, \mathrm{NCCCH}\right), 7.89$ (dt, $J=8.2,1.0 \mathrm{~Hz}, 1 \mathrm{H}, \mathrm{NCHCHCCH}$ ), $7.79-7.65$ (m, 2H, ArH), 7.55 (ddd, $J=8.3,6.9,1.3 \mathrm{~Hz}, 1 \mathrm{H}, \mathrm{NCHCHCCHCH}), 7.19(\mathrm{dd}, J=5.2,1.4$ $\mathrm{Hz}, 1 \mathrm{H}, \mathrm{OCNCHCH}), 7.05$ (dd, $J=1.4,0.8 \mathrm{~Hz}, 1 \mathrm{H}, \mathrm{OCCH}), 4.02\left(\mathrm{~s}, 3 \mathrm{H}, \mathrm{OCH}_{3}\right) \mathrm{ppm}$.

${ }^{13} \mathrm{C}$ NMR (126 MHz, Chloroform- $d$ ) $\delta 164.5(\mathrm{C}), 158.2(\mathrm{C}), 150.0(\mathrm{C}), 147.2(\mathrm{OCNCH}), 142.4(\mathrm{NCH})$, 136.9 (app. d, $J=1.3 \mathrm{~Hz}, \mathrm{CH}_{3} \mathrm{OC}$ ), $130.4(\mathrm{CH}), 127.8$ ( $\left.\mathrm{NCHCHCCHCH}\right), 127.3$ (NCHCHCCH), 126.8 $(\mathrm{NCCCH}), 126.4(\mathrm{C}), 121.0(\mathrm{CH}), 118.1(\mathrm{OCNCHCH}), 111.9(\mathrm{OCCH}), 53.8\left(\mathrm{OCH}_{3}\right) \mathrm{ppm}$. 
HRMS (ESI-TOF) $m / z$ : [M+H] $]^{+}$Calcd for $\mathrm{C}_{15} \mathrm{H}_{14} \mathrm{~N}_{2} \mathrm{O}: 237.1022$; found: 237.1020.

\section{4-(Trifluoromethyl)-2,4'-bipyridine 19}<smiles>FC(F)(F)c1ccnc(-c2ccncc2)c1</smiles>

Product 19 was synthesised via General procedure D using 4-iodopyridine (93 $\mathrm{mg}, 0.45 \mathrm{mmol}$ ) and sulfonium salt 1c $(148.8 \mathrm{mg}, 0.3 \mathrm{mmol})$. The Grignard reagent was formed at $0{ }^{\circ} \mathrm{C}$ for $30 \mathrm{~min}$ and the ligand coupling reaction was stirred at $0{ }^{\circ} \mathrm{C}$ warming to rt. Purification by $\mathrm{FCC}\left(50 \% \mathrm{Et}_{2} \mathrm{O}\right.$ in pentane) gave bipyridine 19 as a yellow oil (37.0 mg, 55\%).

TLC: $R_{\mathrm{f}}=0.27\left(25 \% \mathrm{Et}_{2} \mathrm{O}\right.$ in pentane)

${ }^{1} \mathrm{H}$ NMR (500 MHz, Chloroform- $d$ ) $\delta 8.93\left(\mathrm{dt}, J=5.0,0.7 \mathrm{~Hz}, 1 \mathrm{H}, \mathrm{NCHCHCCF}_{3}\right), 8.83-8.71(\mathrm{~m}, 2 \mathrm{H}$, ArH), 7.99 (dt, $\left.J=1.6,0.7 \mathrm{~Hz}, 1 \mathrm{H}, \mathrm{CF}_{3} \mathrm{CCH}\right), 7.96-7.91$ (m, 2H, ArH), 7.56 (ddd, $J=5.0,1.6,0.7$ $\left.\mathrm{Hz}, 1 \mathrm{H}, \mathrm{NCHCHCCF}_{3}\right) \mathrm{ppm}$.

${ }^{13} \mathrm{C}$ NMR (126 MHz, Chloroform- $d$ ) $\delta 156.3(\mathrm{NCC}), 151.3\left(\mathrm{NCHCHCCF}_{3}\right), 150.8(\mathrm{CH}), 145.1(\mathrm{NCC})$, $139.7\left(\mathrm{q}, J=34.2 \mathrm{~Hz}, \mathrm{CF}_{3} C\right), 122.8\left(\mathrm{q}, J=273.2 \mathrm{~Hz}, \mathrm{CF}_{3}\right), 121.2(\mathrm{CH}), 119.4\left(\mathrm{q}, J=3.5 \mathrm{~Hz}, \mathrm{CF}_{3} \mathrm{CCH}\right)$, $116.6\left(\mathrm{q}, J=3.7 \mathrm{~Hz}, \mathrm{CF}_{3} \mathrm{CCH}\right) \mathrm{ppm}$.

${ }^{19} \mathrm{~F}$ NMR $\left(470 \mathrm{MHz}, \mathrm{CDCl}_{3}\right) \delta-64.8 \mathrm{ppm}$.

HRMS (ESI-TOF) $m / z$ : $[\mathrm{M}+\mathrm{H}]^{+}$Calcd for $\mathrm{C}_{11} \mathrm{H}_{8} \mathrm{~F}_{3} \mathrm{~N}_{2}: 225.0634$; found: 225.0635 .

\section{2'-Methoxy-4-(trifluoromethyl)-2,4'-bipyridine 20}<smiles>COc1cc(-c2cc(C(F)(F)F)ccn2)ccn1</smiles>

Product 20 was synthesised via General procedure D using 4-bromo-2-methoxypyridine (84.6 mg, 0.45 mmol) and sulfonium salt 1 c $(148.8 \mathrm{mg}, 0.3 \mathrm{mmol})$. The Grignard was formed at $40{ }^{\circ} \mathrm{C}$ for $2 \mathrm{~h}$ and the ligand coupling reaction was stirred at rt. Purification by $\mathrm{FCC}\left(15 \% \mathrm{Et}_{2} \mathrm{O}\right.$ in pentane) gave bipyridine 20 as a white solid (49.0 mg, 64\%).

TLC: $R_{\mathrm{f}}=0.46\left(20 \% \mathrm{Et}_{2} \mathrm{O}\right.$ in pentane $)$.

${ }^{1} \mathrm{H}$ NMR (500 MHz, Chloroform- $d$ ) $\delta 8.91\left(\mathrm{dt}, J=5.0,0.8 \mathrm{~Hz}, 1 \mathrm{H}, \mathrm{NCHCHCCF}_{3}\right), 8.31(\mathrm{dd}, J=5.4$, $0.8 \mathrm{~Hz}, 1 \mathrm{H}, \mathrm{OCNCH}), 7.94\left(\mathrm{dt}, J=1.6,0.8 \mathrm{~Hz}, 1 \mathrm{H}, \mathrm{F}_{3} \mathrm{CCCH}\right), 7.54(\mathrm{ddd}, \mathrm{J}=5.0,1.7,0.8 \mathrm{~Hz}$, 
$\mathrm{NCHCHCCF}_{3}$ ), 7.50 (dd, J = 5.4, $\left.1.5 \mathrm{~Hz}, \mathrm{OCNCHCH}\right), 7.37$ (dd, $\left.J=1.5,0.7 \mathrm{~Hz}, 1 \mathrm{H}, \mathrm{OCCH}\right), 4.00$ (s, $\left.3 \mathrm{H}, \mathrm{OCH}_{3}\right) \mathrm{ppm}$.

${ }^{13} \mathrm{C}$ NMR (126 MHz, Chloroform- $\left.d\right) \delta 165.4\left(\mathrm{H}_{3} \mathrm{COC}\right), 156.4\left(\mathrm{~F}_{3} \mathrm{CCCHCN}\right), 151.1\left(\mathrm{NCHCHCCF}_{3}\right)$, $148.0(\mathrm{OCNCH}), 147.98$ (NCC), 139.6 (q, $\left.J=34.3 \mathrm{~Hz}, \mathrm{CF}_{3} C\right), 122.9$ (q, $\left.J=273.2 \mathrm{~Hz}, \mathrm{CF}_{3}\right), 119.3$ (q, $\left.J=3.6 \mathrm{~Hz}, \mathrm{CF}_{3} \mathrm{CCHCH}\right), 116.7$ (q, $\left.J=3.7 \mathrm{~Hz}, \mathrm{CF}_{3} \mathrm{CCHC}\right), 114.6(\mathrm{OCNCHCH}), 108.7(\mathrm{OCCH}), 53.9$ $\left(\mathrm{OCH}_{3}\right) \mathrm{ppm}$.

HRMS (ESI-TOF) $m / z$ : [M+H] $]^{+}$Calcd for $\mathrm{C}_{12} \mathrm{H}_{10} \mathrm{~F}_{3} \mathrm{~N}_{2} \mathrm{O}$ : 254.0661; found: 254.0676 .

2',6'-Dichloro-3-methyl-2,4'-bipyridine 21<smiles>Cc1cccnc1-c1cc(Cl)nc(Cl)c1</smiles>

Product 21 was synthesised via General procedure E using 2,6-dichloropyridine ( $50.1 \mu \mathrm{L}, 0.45 \mathrm{mmol}$ ) and sulfonium salt 1d (132.4 $\mathrm{mg}, 0.3 \mathrm{mmol})$. The Grignard reagent was formed at $\mathrm{rt}$ for $10 \mathrm{~min}$ and the ligand coupling reaction was stirred at $\mathrm{rt}$. Purification by FCC ( $5 \% \mathrm{Et}_{2} \mathrm{O}$ in pentane) gave bipyridine 21 as a white solid (46.2 $\mathrm{mg}, 64 \%)$.

TLC: $R_{\mathrm{f}}=0.35\left(20 \% \mathrm{Et}_{2} \mathrm{O}\right.$ in pentane $)$.

${ }^{1} \mathrm{H}$ NMR (500 MHz, Chloroform- $d$ ) $\delta 8.54$ (ddd, $\left.J=4.7,1.6,0.7 \mathrm{~Hz}, 1 \mathrm{H}, \mathrm{NCH}\right), 7.64$ (ddd, $J=7.8,1.7$, $0.8 \mathrm{~Hz}, 1 \mathrm{H}, \mathrm{NCHCHCH}), 7.45$ (s, 2H, ArH), 7.29 (dd, $J=7.8,4.7 \mathrm{~Hz}, 1 \mathrm{H}, \mathrm{NCHCH}), 2.39\left(\mathrm{~s}, 3 \mathrm{H}, \mathrm{CH}_{3}\right)$ ppm.

${ }^{13} \mathrm{C}$ NMR (126 MHz, Chloroform- $d$ ) $\delta 153.5(\mathrm{C}), 153.4(\mathrm{C}), 150.7\left(\mathrm{CH}_{3} \mathrm{C}\right), 147.8(\mathrm{NCH}), 139.4$ ( $\mathrm{NCHCHCH}), 131.4(\mathrm{NCCl}), 124.1(\mathrm{NCHCH}), 123.2(\mathrm{NCClCH}), 19.8\left(\mathrm{CH}_{3}\right) \mathrm{ppm}$.

HRMS (ESI-TOF) $m / z$ : $[\mathrm{M}+\mathrm{H}]^{+}$Calcd for $\mathrm{C}_{11} \mathrm{H}_{9} \mathrm{Cl}_{2} \mathrm{~N}_{2}: 239.0137,240.0167,241.0109,242.0138$ and 243.0082; found: 239.0140, 240.0173, 241.0110, 242.0142 and 243.0080.

\section{2'-Fluoro-6-(trifluoromethyl)-2,4'-bipyridine 22}<smiles>Fc1cc(-c2cccc(C(F)(F)F)n2)ccn1</smiles>

Product 22 was synthesised via General procedure D using 4-bromo-2-fluoropyridine (46 $\mu \mathrm{L}, 0.45$ mmol) and sulfonium salt $1 \mathbf{i}(148.6 \mathrm{mg}, 0.3 \mathrm{mmol})$. The Grignard reagent was formed at $\mathrm{rt}$ for $1 \mathrm{~h}$ and 
the ligand coupling reaction was stirred at $\mathrm{rt}$. Purification by $\mathrm{FCC}\left(20 \% \mathrm{Et}_{2} \mathrm{O}\right.$ in pentane) gave bipyridine $\mathbf{2 2}$ as a white solid (41.1 $\mathrm{mg}, 57 \%)$.

TLC: $R_{\mathrm{f}}=0.14\left(20 \% \mathrm{Et}_{2} \mathrm{O}\right.$ in pentane).

${ }^{1} \mathrm{H}$ NMR (500 MHz, Chloroform- $d$ ) $\delta 8.35(\mathrm{~d}, J=5.3 \mathrm{~Hz}, 1 \mathrm{H}), 8.04(\mathrm{t}, J=7.8 \mathrm{~Hz}, 1 \mathrm{H}), 7.99(\mathrm{~d}, J=8.0$ $\mathrm{Hz}, 1 \mathrm{H}), 7.85-7.82(\mathrm{~m}, 1 \mathrm{H}), 7.76(\mathrm{~d}, J=7.7 \mathrm{~Hz}, 1 \mathrm{H}), 7.62(\mathrm{~s}, 1 \mathrm{H})$.

${ }^{13} \mathrm{C}$ NMR (126 MHz, Chloroform- $d$ ) $\delta 164.9$ (d, $\left.J=238.7 \mathrm{~Hz}, \mathrm{CF}\right), 153.9$ (d, $\left.J=3.3 \mathrm{~Hz}, \mathrm{C}\right), 150.5$ (d, $J=8.3 \mathrm{~Hz}, \mathrm{C}), 148.9\left(\mathrm{q}, J=35.0 \mathrm{~Hz}, C C F_{3}\right), 148.7(\mathrm{~d}, J=15.2 \mathrm{~Hz}, \mathrm{CH}), 139.0(\mathrm{CH}), 123.5(\mathrm{CH}), 121.3$ (q, $\left.J=274.4 \mathrm{~Hz}, \mathrm{CF}_{3}\right), 121.0(\mathrm{q}, J=2.8 \mathrm{~Hz}, \mathrm{CH}), 119.0(\mathrm{~d}, J=3.9 \mathrm{~Hz}, \mathrm{CH}), 107.4(\mathrm{~d}, J=39.1 \mathrm{~Hz}$, $\mathrm{CH})$.

${ }^{19}$ F NMR (470 MHz, Chloroform- $d$ ) $\delta-67.2,-68.2$.

HRMS (ESI-TOF) $m / z$ : [M+H] $]^{+}$Calcd for $\mathrm{C}_{11} \mathrm{H}_{7} \mathrm{~F}_{4} \mathrm{~N}_{2}$ : 243.0540; Found: 243.0542 .

\section{5-Bromo-2'-fluoro-2,4'-bipyridine 23}

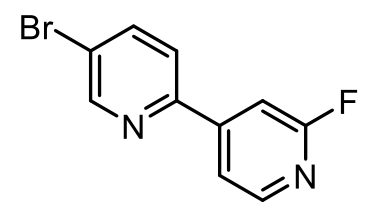

Product 23 was synthesised via General procedure D using 4-bromo-2-fluoropyridine (46 $\mu \mathrm{L}, 0.45$ mmol) and sulfonium salt $1 \mathbf{k}(151.9 \mathrm{mg}, 0.3 \mathrm{mmol})$. The Grignard reagent was formed at $45^{\circ} \mathrm{C}$ for $1 \mathrm{~h}$ and the ligand coupling reaction was stirred at $45{ }^{\circ} \mathrm{C}$ for $4.5 \mathrm{~h}$. Purification by $\mathrm{FCC}\left(40 \% \mathrm{Et}_{2} \mathrm{O}\right.$ in pentane) gave bipyridine $\mathbf{2 3}$ as a white solid (43.3 $\mathrm{mg}, 57 \%$ ).

TLC: $R_{\mathrm{f}}=0.44\left(40 \% \mathrm{Et}_{2} \mathrm{O}\right.$ in pentane).

${ }^{1} \mathrm{H}$ NMR (400 MHz, Chloroform- $d$ ) $\delta 8.80(\mathrm{dd}, J=2.3,0.6 \mathrm{~Hz}, 1 \mathrm{H}), 8.33(\mathrm{~d}, J=5.3 \mathrm{~Hz}, 1 \mathrm{H}), 7.97$ (dd, $J=8.6,2.3 \mathrm{~Hz}, 1 \mathrm{H}), 7.75(\mathrm{dt}, J=5.4,1.7 \mathrm{~Hz}, 1 \mathrm{H}), 7.69(\mathrm{~d}, J=8.3 \mathrm{~Hz}, 1 \mathrm{H}), 7.54(\mathrm{t}, J=1.7 \mathrm{~Hz}, 1 \mathrm{H})$.

${ }^{13} \mathrm{C}$ NMR (126 MHz, Chloroform- $d$ ) $\delta 164.9(\mathrm{~d}, J=238.4 \mathrm{~Hz}, \mathrm{CF}), 151.9(\mathrm{~d}, J=3.8 \mathrm{~Hz}, \mathrm{C}), 151.5$ $(\mathrm{CH}), 151.0(\mathrm{~d}, J=8.5 \mathrm{~Hz}, \mathrm{C}), 148.6(\mathrm{~d}, J=15.2 \mathrm{~Hz}, \mathrm{CH}), 139.9(\mathrm{CH}), 122.2(\mathrm{CH}), 122.0(\mathrm{CBr}), 118.7$ $(\mathrm{d}, J=3.8 \mathrm{~Hz}, \mathrm{CH}), 107.0(\mathrm{~d}, J=39.1 \mathrm{~Hz}, \mathrm{CH})$.

${ }^{19} \mathrm{~F}$ NMR (376 MHz, Chloroform- $d$ ) $\delta$-67.4.

HRMS (ESI-TOF) $m / z$ : $[\mathrm{M}+\mathrm{H}]^{+}$Calcd for $\mathrm{C}_{10} \mathrm{H}_{7} \mathrm{BrFN}_{2}$ : 252.9771, 254.9751; Found: 252.9772, 254.9751 . 


\section{2-(Pyridin-3-yl)pyrimidine 24}<smiles>c1cnc(-c2cccnc2)nc1</smiles>

Product 24 was synthesised via General procedure D using 3-iodopyridine (92.3 mg, $0.45 \mathrm{mmol})$ and sulfonium salt $1 \mathbf{m}(128.5 \mathrm{mg}, 0.3 \mathrm{mmol})$. The Grignard reagent was formed at $\mathrm{rt}$ for $45 \mathrm{~min}$ and the ligand coupling reaction was stirred at $\mathrm{rt}$. Purification by $\mathrm{FCC}\left(80 \% \mathrm{Et}_{2} \mathrm{O}\right.$ in pentane) gave bisheteroaryl 24 as a brown solid (33.1 $\mathrm{mg}, 70 \%)$.

TLC: $R_{\mathrm{f}}=0.24\left(80 \% \mathrm{Et}_{2} \mathrm{O}\right.$ in pentane $)$

${ }^{1} \mathrm{H}$ NMR (400 MHz, Chloroform- $d$ ) $\delta 9.65(\mathrm{~d}, J=2.2 \mathrm{~Hz}, 1 \mathrm{H}), 8.84(\mathrm{~d}, J=4.8 \mathrm{~Hz}, 2 \mathrm{H}), 8.74-8.66$ (m, 2H), $7.45-7.40(\mathrm{~m}, 1 \mathrm{H}), 7.26(\mathrm{t}, J=4.9 \mathrm{~Hz}, 1 \mathrm{H})$.

${ }^{13} \mathrm{C}$ NMR (101 MHz, Chloroform- $d$ ) $\delta 163.2,157.5,151.5,150.0,135.6,133.2,123.5,119.9$.

Spectra were consistent with literature data. ${ }^{13}$

2-(6-(Trifluoromethyl)pyridin-2-yl)pyrimidine 25<smiles>FC(F)(F)c1cccc(-c2ncccn2)n1</smiles>

Product 25 was synthesised via General procedure D using 2-bromo-6-(trifluoromethyl)pyridine (101.7 $\mathrm{mg}, 0.45 \mathrm{mmol})$ and sulfonium salt $1 \mathbf{m}(128.5 \mathrm{mg}, 0.3 \mathrm{mmol})$. The Grignard reagent was formed at 45 ${ }^{\circ} \mathrm{C}$ for $2 \mathrm{~h}$ and the ligand coupling reaction was stirred at $-78{ }^{\circ} \mathrm{C}$. Purification by $\mathrm{FCC}\left(60 \% \mathrm{Et}_{2} \mathrm{O}\right.$ in pentane) gave bis-heteroaryl 25 as an orange solid (27.2 $\mathrm{mg}, 40 \%)$.

TLC: $R_{\mathrm{f}}=0.16\left(60 \% \mathrm{Et}_{2} \mathrm{O}\right.$ in pentane $)$

${ }^{1} \mathrm{H}$ NMR (400 MHz, Chloroform- $d$ ) $\delta 8.97$ (d, $\left.J=4.8 \mathrm{~Hz}, 2 \mathrm{H}\right), 8.70$ (d, $\left.J=8.0 \mathrm{~Hz}, 1 \mathrm{H}\right), 8.06$ (t, $J=7.9$ $\mathrm{Hz}, 1 \mathrm{H}), 7.81(\mathrm{~d}, J=7.8 \mathrm{~Hz}, 1 \mathrm{H}), 7.37$ (t, $J=4.8 \mathrm{~Hz}, 1 \mathrm{H})$.

${ }^{13} \mathrm{C}$ NMR (101 MHz, Chloroform- $d$ ) $\delta 162.8(\mathrm{C}), 158.0(\mathrm{CH}), 155.6(\mathrm{C}), 148.8\left(\mathrm{q}, J=34.8 \mathrm{~Hz}, \mathrm{CCF}_{3}\right)$, $138.6(\mathrm{CH}), 126.5(\mathrm{CH}), 121.7$ (q, $J=3.0 \mathrm{~Hz}, \mathrm{CH}), 121.6\left(\mathrm{~d}, J=274.6 \mathrm{~Hz}, \mathrm{CF}_{3}\right), 121.0(\mathrm{CH})$.

${ }^{19} \mathrm{~F}$ NMR (376 MHz, Chloroform- $d$ ) $\delta-67.5$.

HRMS (ESI-TOF) $m / z$ : [M+H] $]^{+}$Calcd for $\mathrm{C}_{10} \mathrm{H}_{7} \mathrm{~F}_{3} \mathrm{~N}_{3}: 226.0587$; Found: 226.0589 . 


\section{2-Bromo-4-(4-(trifluoromethyl)pyridin-2-yl)pyrimidine 26}<smiles>FC(F)(F)c1ccnc(-c2ccnc(Br)n2)c1</smiles>

Product 26 was synthesised via General procedure A using 2,4-dibromopyrimidine (71.5 mg, 0.45 $\mathrm{mmol})$ and sulfonium salt $1 \mathrm{c}(148.8 \mathrm{mg}, 0.3 \mathrm{mmol})$. The Grignard reagent was formed at $-45^{\circ} \mathrm{C}$ for 2 h. Purification by FCC (10\% $\mathrm{Et}_{2} \mathrm{O}$ in pentane) gave bis-heteroaryl 26 as a white solid $(58.1 \mathrm{mg}, 64 \%)$. TLC: $R_{\mathrm{f}}=0.35\left(20 \% \mathrm{Et}_{2} \mathrm{O}\right.$ in pentane $)$.

${ }^{1} \mathrm{H}$ NMR (500 MHz, Chloroform- $d$ ) $\delta 8.90$ (dt, $\left.J=5.0,0.7 \mathrm{~Hz}, 1 \mathrm{H}\right), 8.73(\mathrm{~d}, J=5.1 \mathrm{~Hz}, 1 \mathrm{H}), 8.72-$ $8.70(\mathrm{~m}, 1 \mathrm{H}), 8.39$ (d, $J=5.0 \mathrm{~Hz}, 1 \mathrm{H}), 7.67$ (ddd, $J=5.0,1.7,0.8 \mathrm{~Hz}, 1 \mathrm{H})$.

${ }^{13} \mathrm{C}$ NMR (126 MHz, Chloroform-d) $\delta 164.5(\mathrm{CBr}), 160.8(\mathrm{CH}), 154.0(\mathrm{C}), 153.4(\mathrm{C}), 150.8(\mathrm{CH})$, 140.05 (q, $\left.J=34.8 \mathrm{~Hz}, C \mathrm{CF}_{3}\right), 122.7$ (t, $\left.J=273.8 \mathrm{~Hz}, \mathrm{CF}_{3}\right), 121.7$ (q, $\left.J=3.4 \mathrm{~Hz}, \mathrm{CH}\right), 118.1$ (q, $J=3.5$ $\mathrm{Hz}, \mathrm{CH}), 116.5(\mathrm{CH})$.

${ }^{19} \mathrm{~F}$ NMR (470 MHz, Chloroform- $\left.d\right) \delta-64.8$.

HRMS (ESI ${ }^{+}$): $[\mathrm{M}+\mathrm{H}]^{+}$Calcd for $\mathrm{C}_{10} \mathrm{H}_{5} \mathrm{BrF}_{3} \mathrm{~N}_{3}$ : 303.9692 and 305.9672; found: 303.9694 and 305.9676.

2-(5-(Trifluoromethyl)pyridin-2-yl)pyrazine 27<smiles>FC(F)(F)c1ccc(-c2cnccn2)nc1</smiles>

Product 27 was synthesised via General procedure E with a slight modification using iodopyrazine (44.4 $\mu \mathrm{L}, 0.45 \mathrm{mmol})$, sulfonium salt $1 \mathrm{e}(148.8 \mathrm{mg}, 0.3 \mathrm{mmol})$ and $n$-butylmagnesium chloride $(0.23 \mathrm{~mL}, 2.0$ $\mathrm{M}$ in ether). The Grignard reagent was formed at $-78{ }^{\circ} \mathrm{C}$ for $30 \mathrm{~min}$ and the ligand coupling reaction was stirred at $-78{ }^{\circ} \mathrm{C}$ for $8 \mathrm{~h}$. Purification by FCC $\left(25 \% \mathrm{Et}_{2} \mathrm{O}\right.$ in pentane) gave bis-heteroaryl 27 as a white solid (39.6 mg, 59\%).

TLC: $R_{\mathrm{f}}=\left(25 \% \mathrm{Et}_{2} \mathrm{O}\right.$ in pentane $)$.

${ }^{1} \mathrm{H}$ NMR (500 MHz, Chloroform- $d$ ) $\delta 9.69(\mathrm{~d}, J=1.4 \mathrm{~Hz}, 1 \mathrm{H}, \mathrm{NCHCN}), 8.97$ (dt, $J=2.0,0.9 \mathrm{~Hz}, 1 \mathrm{H}$, $\left.\mathrm{NCHCCF}_{3}\right), 8.73-8.58(\mathrm{~m}, 2 \mathrm{H}, \mathrm{ArH}), 8.52\left(\mathrm{dt}, J=8.3,0.8 \mathrm{~Hz}, 1 \mathrm{H}, \mathrm{F}_{3} \mathrm{CCCHCH}\right), 8.15-8.03(\mathrm{~m}, 1 \mathrm{H}$, $\left.\mathrm{F}_{3} \mathrm{CCCHCH}\right) \mathrm{ppm}$. 
${ }^{13} \mathrm{C}$ NMR (126 MHz, Chloroform- $d$ ) $\delta 157.6$ (app. d, $\left.J=2.4 \mathrm{~Hz}, \mathrm{NCCCH}\right), 149.9$ (NCCCH), 146.6 (q, $\left.J=3.9 \mathrm{~Hz}, \mathrm{NCHCCF}_{3}\right), 145.6(\mathrm{CH}), 143.92(\mathrm{CH}), 143.91(\mathrm{NCHCN}), 134.4\left(\mathrm{q}, J=3.5 \mathrm{~Hz}, \mathrm{~F}_{3} \mathrm{CCHCH}\right)$, $127.1\left(\mathrm{q}, J=33.0 \mathrm{~Hz}, \mathrm{CF}_{3} C\right), 123.6\left(\mathrm{q}, J=272.4 \mathrm{~Hz}, \mathrm{CF}_{3}\right), 121.2(\mathrm{CNCHCHN}) \mathrm{ppm}$.

${ }^{19} \mathrm{~F}$ NMR (470 MHz, Chloroform- $d$ ) $\delta-62.5 \mathrm{ppm}$.

HRMS (ESI-TOF) $m / z$ : [M+H] $]^{+}$Calcd for $\mathrm{C}_{10} \mathrm{H}_{7} \mathrm{~F}_{3} \mathrm{~N}_{3}$ : 226.0587; found: 226.0588 .

\section{2-(Benzo $[b]$ thiophen-2-yl)pyridine 28}

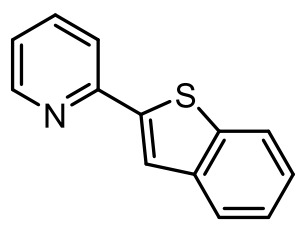

Product 28 was synthesised via General procedure E using benzothiophene $(60.0 \mathrm{mg}, 0.45 \mathrm{mmol})$ and sulfonium salt 1a (128.2 mg, $0.3 \mathrm{mmol})$. The Grignard reagent was formed at $\mathrm{rt}$ for $24 \mathrm{~h}$ and the ligand coupling reaction was stirred at $45{ }^{\circ} \mathrm{C}$. Purification by FCC $\left(100 \% \mathrm{Et}_{2} \mathrm{O}\right)$ gave bis-heteroaryl 28 as a yellow solid (44.3 mg, 70\%).

TLC: $R_{\mathrm{f}}=0.05\left(100 \% \mathrm{Et}_{2} \mathrm{O}\right)$

${ }^{1} \mathrm{H}$ NMR (400 MHz, Chloroform- $d$ ) $\delta 8.64$ (dd, $\left.J=4.5,1.6 \mathrm{~Hz}, 1 \mathrm{H}\right), 7.90-7.85$ (m, 1H), $7.83(\mathrm{~s}, 1 \mathrm{H})$, $7.82-7.77(\mathrm{~m}, 2 \mathrm{H}), 7.72(\mathrm{td}, J=7.7,1.9 \mathrm{~Hz}, 1 \mathrm{H}), 7.39-7.32(\mathrm{~m}, 2 \mathrm{H}), 7.20(\mathrm{dd}, J=7.4,4.8 \mathrm{~Hz}, 1 \mathrm{H})$.

${ }^{13} \mathrm{C}$ NMR (101 MHz, Chloroform- $d$ ) $\delta 152.7,149.8,144.9,140.8,140.6,136.7,125.2,124.6,124.2$, $122.7,121.2,119.7$.

Spectra were consistent with literature data. ${ }^{14}$

\section{3,5-Dimethyl-4-(pyridin-2-yl)isoxazole 29}<smiles>Cc1noc(C)c1-c1ccccn1</smiles>

Product 29 was synthesised via General procedure D using 4-iodo-3,5-dimethylisoxazole (100.4 mg, $0.45 \mathrm{mmol})$ and sulfonium salt $1 \mathrm{a}(128.2 \mathrm{mg}, 0.3 \mathrm{mmol})$. The Grignard reagent was formed at $45^{\circ} \mathrm{C}$ for $2 \mathrm{~h}$ and the ligand coupling reaction was stirred at $45^{\circ} \mathrm{C}$. Purification by FCC ( $30 \% \mathrm{Et}_{2} \mathrm{O}$ in pentane) gave bis-heteroaryl 29 contaminated with an impurity (8\%) as a yellow oil (27.0 mg, 48\%).

TLC: $R_{\mathrm{f}}=0.24\left(30 \% \mathrm{Et}_{2} \mathrm{O}\right.$ in pentane).

${ }^{1} \mathrm{H}$ NMR (400 MHz, Chloroform-d) $\delta 8.69-8.65(\mathrm{~m}, 1 \mathrm{H}), 7.74(\mathrm{td}, J=7.8,1.9 \mathrm{~Hz}, 1 \mathrm{H}), 7.32(\mathrm{~d}, J=$ $7.9 \mathrm{~Hz}, 1 \mathrm{H}), 7.22(\mathrm{dd}, J=7.6,4.9 \mathrm{~Hz}, 1 \mathrm{H}), 2.56\left(\mathrm{~s}, 3 \mathrm{H}, \mathrm{CH}_{3}\right), 2.42\left(\mathrm{~s}, 3 \mathrm{H}, \mathrm{CH}_{3}\right)$. 
${ }^{13} \mathrm{C}$ NMR (101 MHz, Chloroform- $d$ ) $\delta 167.5,158.8,151.0,150.1,136.6,123.0,121.9,116.2,12.5$ $\left(\mathrm{CH}_{3}\right), 11.6\left(\mathrm{CH}_{3}\right)$.

Spectra were consistent with literature data. ${ }^{15}$

\section{2-Phenylpyridine 30}<smiles>c1ccc(-c2ccccn2)cc1</smiles>

Product 30 was synthesised via General procedure D using phenylmagnesium bromide (2.6 M in diethyl ether, $0.17 \mathrm{~mL}, 0.45 \mathrm{mmol})$ and sulfonium salt $1 \mathrm{a}(128.2 \mathrm{mg}, 0.3 \mathrm{mmol})$. The ligand coupling reaction was stirred at rt. Purification by FCC $\left(80 \% \mathrm{Et}_{2} \mathrm{O}\right.$ in pentane) gave product $\mathbf{3 0}$ as a yellow oil $(32.1 \mathrm{mg}$, $69 \%)$.

TLC: $R_{\mathrm{f}}=0.45\left(20 \% \mathrm{Et}_{2} \mathrm{O}\right.$ in pentane).

${ }^{1} \mathrm{H}$ NMR (400 MHz, Chloroform- $d$ ) $\delta 8.70$ (dt, $\left.J=4.7,1.4 \mathrm{~Hz}, 1 \mathrm{H}\right), 8.02-7.98(\mathrm{~m}, 2 \mathrm{H}), 7.78-7.70$ $(\mathrm{m}, 2 \mathrm{H}), 7.52-7.45(\mathrm{~m}, 2 \mathrm{H}), 7.45-7.39(\mathrm{~m}, 1 \mathrm{H}), 7.23$ (ddd, $J=6.0,4.7,2.4 \mathrm{~Hz}, 1 \mathrm{H})$.

${ }^{13} \mathrm{C}$ NMR (101 MHz, Chloroform- $d$ ) $\delta$ 157.6, 149.8, 139.5, 136.9, 129.1, 128.9, 127.0, 122.2, 120.7. Spectra were consistent with literature data. ${ }^{16}$

\section{Synthesis of caerulomycin $E$ and $A$}

\section{6-Bromo-4-methoxy-2,2'-bipyridine 36}<smiles>COc1cc(Br)nc(-c2ccccn2)c1</smiles>

Product 36 was synthesised via General procedure D using 2,6-dibromo-4-methoxypyridine $(88.0 \mathrm{mg}$, $0.33 \mathrm{mmol})$ and sulfonium salt $1 \mathrm{a}(128.2 \mathrm{mg}, 0.3 \mathrm{mmol})$. The Grignard reagent was formed at $45{ }^{\circ} \mathrm{C}$ for $2 \mathrm{~h}$ and the ligand coupling reaction was stirred at $\mathrm{rt}$ for $1 \mathrm{~h}$ and then at $30^{\circ} \mathrm{C}$ for $1 \mathrm{~h}$. Purification by $\mathrm{FCC}\left(50 \% \mathrm{Et}_{2} \mathrm{O}\right.$ in pentane) gave bipyridine 36 as a white solid $(73.3 \mathrm{mg}, 92 \%)$.

TLC: $R_{\mathrm{f}}=0.50\left(50 \% \mathrm{Et}_{2} \mathrm{O}\right.$ in pentane).

${ }^{1} \mathrm{H}$ NMR (400 MHz, Chloroform- $d$ ) $\delta 8.64(\mathrm{dd}, J=4.7,1.7 \mathrm{~Hz}, 1 \mathrm{H}), 8.39$ (dt, $\left.J=8.1,1.3 \mathrm{~Hz}, 1 \mathrm{H}\right), 7.94$ $(\mathrm{d}, J=2.2 \mathrm{~Hz}, 1 \mathrm{H}), 7.80(\mathrm{td}, J=7.7,1.8 \mathrm{~Hz}, 1 \mathrm{H}), 7.31(\mathrm{ddd}, J=7.6,4.8,1.2 \mathrm{~Hz}, 1 \mathrm{H}), 7.01(\mathrm{~d}, J=2.3$ $\mathrm{Hz}, 1 \mathrm{H}), 3.93$ (s, 3H, OMe). 
${ }^{13} \mathrm{C}$ NMR (101 MHz, Chloroform-d) $\delta 167.9,158.4,154.5,149.2,142.3,137.1,124.5,121.8,114.1$, 106.2, 56.0 (OMe).

Spectra were consistent with literature data. ${ }^{17}$

4-Methoxy-[2,2'-bipyridine]-6-carbaldehyde, caerulomycin E 37<smiles>COc1cc(C=O)nc(-c2ccccn2)c1</smiles>

An oven-dried crimptop vial was evacuated and purged with $\mathrm{N}_{2}$ three times. A solution of $n$-BuLi $(0.15$ $\mathrm{mL}, 0.31 \mathrm{mmol}, 2.1 \mathrm{M}$ in hexanes $)$ in $\operatorname{dry}_{\mathrm{Et}} \mathrm{O}(1.0 \mathrm{~mL})$ was prepared and cooled to $-78{ }^{\circ} \mathrm{C}$. A solution of 6-bromo-4-methoxy-2,2'-bipyridine $(73 \mathrm{mg}, 0.28 \mathrm{mmol})$ in dry $\mathrm{Et}_{2} \mathrm{O}(0.5 \mathrm{~mL})$ was prepared in a separate oven-dried crimptop vial and added dropwise to the $n$-BuLi solution. Stirring was continued at $-78{ }^{\circ} \mathrm{C}$ for $30 \mathrm{~min}$. Dimethylformamide $(42 \mu \mathrm{L}, 0.54 \mathrm{mmol})$ in dry $\mathrm{Et}_{2} \mathrm{O}(0.3 \mathrm{~mL})$ was prepared in a separate oven-dried crimptop vial and then added dropwise to the reaction flask and stirred for $90 \mathrm{~min}$. The reaction was quenched at $-78^{\circ} \mathrm{C}$ with sat. $\mathrm{NH}_{4} \mathrm{Cl}(2 \mathrm{~mL})$. The reaction mixture was extracted with $\mathrm{Et}_{2} \mathrm{O}(3 \times 10 \mathrm{~mL})$, the combined organic layers were washed with water $(20 \mathrm{~mL})$ and brine $(20 \mathrm{~mL})$, and then dried over anhydrous $\mathrm{Na}_{2} \mathrm{SO}_{4}$. Purification by $\mathrm{FCC}\left(10 \% \mathrm{MeOH}\right.$ in $\left.\mathrm{CH}_{2} \mathrm{Cl}_{2}\right)$ gave caerulomycin E as a yellow solid (35.6 mg, 60\%).

TLC: $R_{\mathrm{f}}=0.08\left(10 \% \mathrm{MeOH}\right.$ in $\left.\mathrm{CH}_{2} \mathrm{Cl}_{2}\right)$.

${ }^{1} \mathrm{H}$ NMR (400 MHz, Chloroform- $d$ ) $\delta 10.13$ (s, 1H, CHO), 8.70 (dt, $J=4.9,1.5 \mathrm{~Hz}, 1 \mathrm{H}$ ), $8.58-8.50$ (m, 1H), 8.19 (d, $J=2.6 \mathrm{~Hz}, 1 \mathrm{H}), 7.86$ (td, $J=7.7,1.8 \mathrm{~Hz}, 1 \mathrm{H}), 7.49$ (d, $J=2.4 \mathrm{~Hz}, 1 \mathrm{H}), 7.36$ (ddd, $J$ $=7.5,4.9,1.2 \mathrm{~Hz}, 1 \mathrm{H}), 4.00(\mathrm{~s}, 3 \mathrm{H}, \mathrm{OMe})$.

${ }^{13} \mathrm{C}$ NMR (101 MHz, Chloroform- $d$ ) $\delta 193.8$ (CHO), 167.8, 158.6, 155.1, 154.3, 149.4, 137.2, 124.5, 121.6, 110.7, 107.8, 56.0 (OMe).

Spectra were consistent with literature data. ${ }^{17}$ 
(E)-4-Methoxy-[2,2'-bipyridine]-6-carbaldehyde oxime, caerulomycin A 38<smiles>COc1cc(/C=N/O)nc(-c2ccccn2)c1</smiles>

Caerulomycin A was prepared following a procedure adapted from Quéguiner and co-workers. ${ }^{17}$ Caerulomycin E (0.025 g, $0.12 \mathrm{mmol})$, hydroxylamine hydrochloride $(0.041 \mathrm{~g}, 0.59 \mathrm{mmol})$, pyridine $(0.041 \mathrm{~mL}, 0.50 \mathrm{mmol})$ and $\mathrm{EtOH}(0.84 \mathrm{~mL})$ were added to a crimptop vial, which was sealed and heated under reflux for $1 \mathrm{~h}$. Solvent was removed under vacuum and purification by FCC $(10 \% \mathrm{MeOH}$ in $\mathrm{CH}_{2} \mathrm{Cl}_{2}$ ) gave caerulomycin $\mathrm{A}$ as a white solid $(24.1 \mathrm{mg}, 90 \%)$.

TLC: $R_{\mathrm{f}}=0.07\left(10 \% \mathrm{MeOH}\right.$ in $\left.\mathrm{CH}_{2} \mathrm{Cl}_{2}\right)$.

${ }^{1} \mathrm{H}$ NMR (400 MHz, DMSO- $\left.d_{6}\right) \delta 11.72(\mathrm{~s}, 1 \mathrm{H}), 8.69$ (ddd, $\left.J=4.8,1.8,0.9 \mathrm{~Hz}, 1 \mathrm{H}\right), 8.39$ (dt, $J=8.0$, $1.1 \mathrm{~Hz}, 1 \mathrm{H}), 8.15$ (s, 1H), 7.96 (td, $J=7.7,1.8 \mathrm{~Hz}, 1 \mathrm{H}), 7.91$ (d, $J=2.5 \mathrm{~Hz}, 1 \mathrm{H}), 7.48$ (ddd, $J=7.5$, 4.8, $1.2 \mathrm{~Hz}, 1 \mathrm{H}), 7.33$ (d, $J=2.5 \mathrm{~Hz}, 1 \mathrm{H}), 3.95$ (s, 3H, OMe).

${ }^{13} \mathrm{C}$ NMR (101 MHz, DMSO- $\left.d_{6}\right) \delta 166.6,156.9,154.5,153.4,149.3,148.8,137.3,124.5,120.7,106.5$, 105.6, 55.6.

Spectra were consistent with literature data. ${ }^{17}$ 


\section{NMR Spectra}

${ }^{1} \mathrm{H}$ NMR (500 MHz, $\mathrm{CDCl}_{3}$ ): 1hi

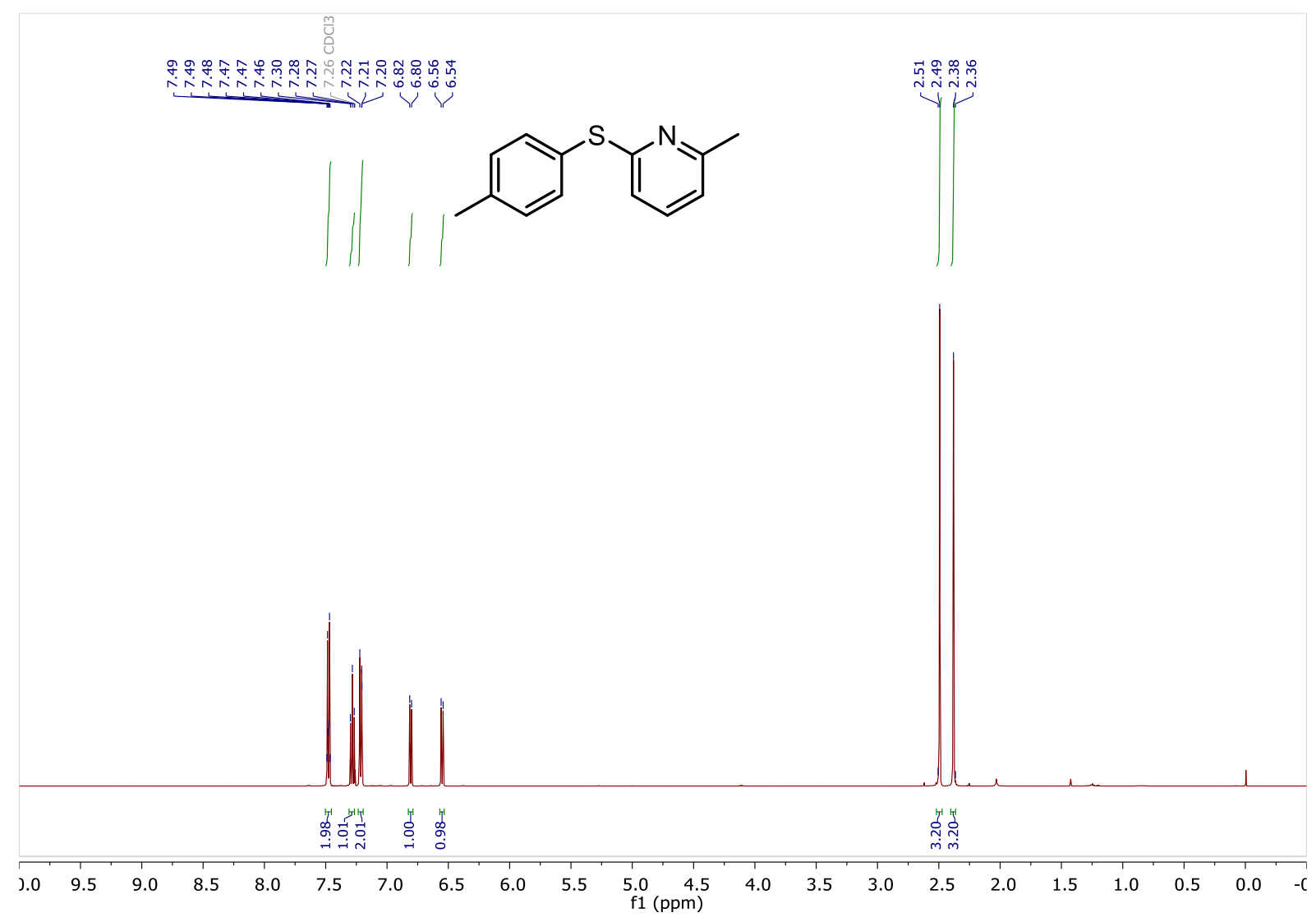

${ }^{13} \mathrm{C}$ NMR (101 MHz, $\left.\mathrm{CDCl}_{3}\right)$ : 1hi

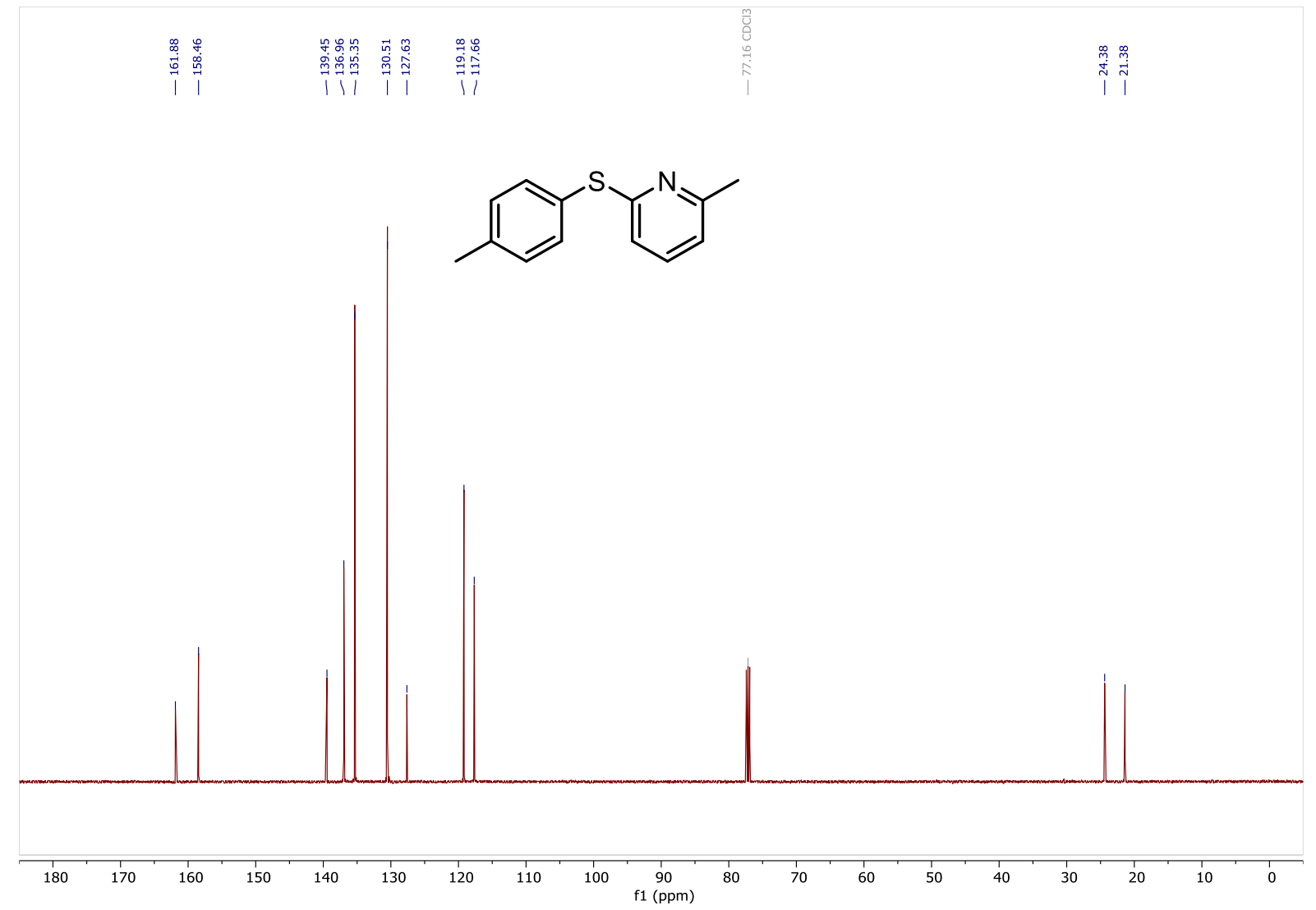


${ }^{1} \mathrm{H}$ NMR (500 MHz, $\mathrm{CDCl}_{3}$ ): 1ii

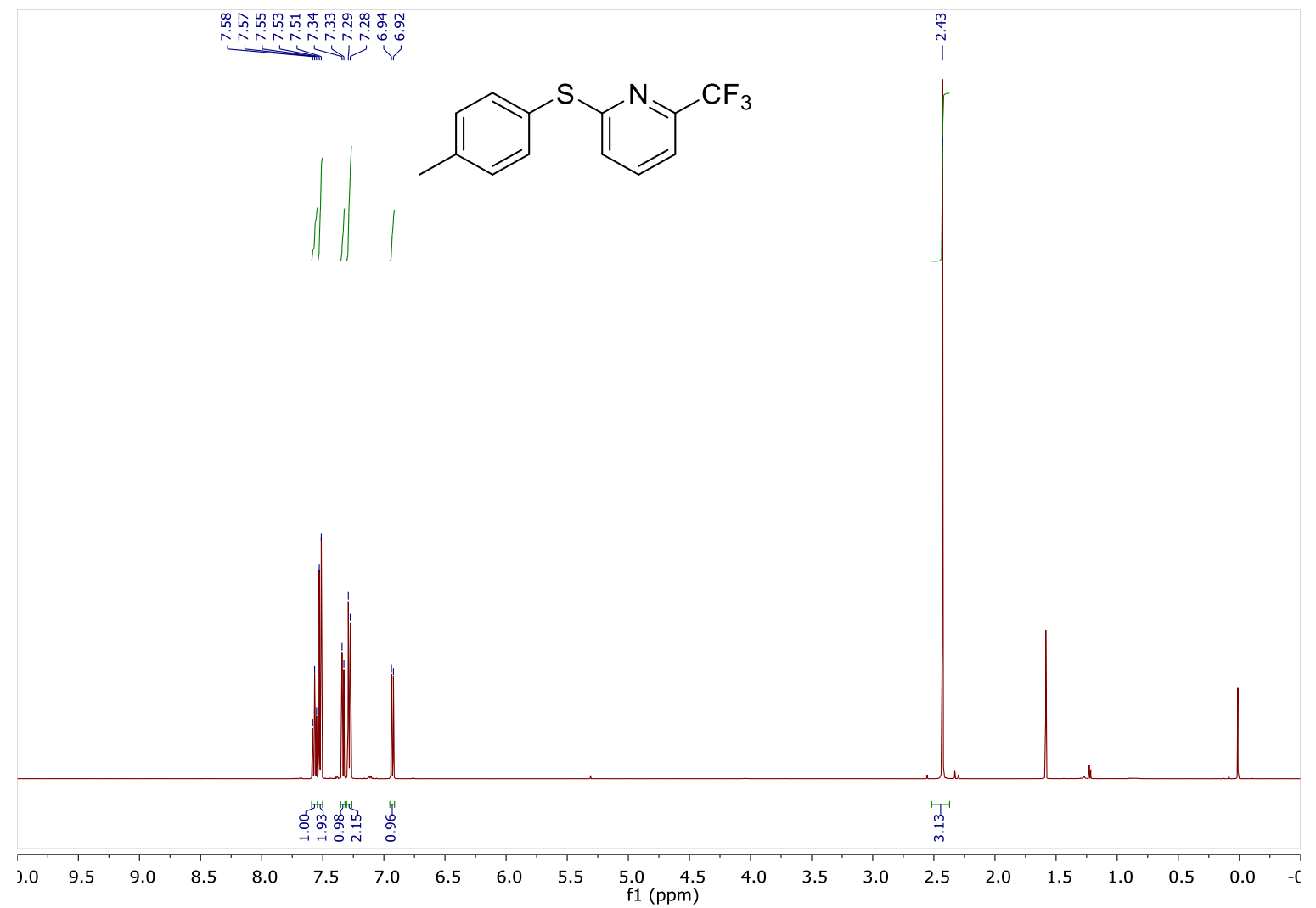

${ }^{13} \mathrm{C}$ NMR (101 MHz, $\left.\mathrm{CDCl}_{3}\right)$ : 1ii

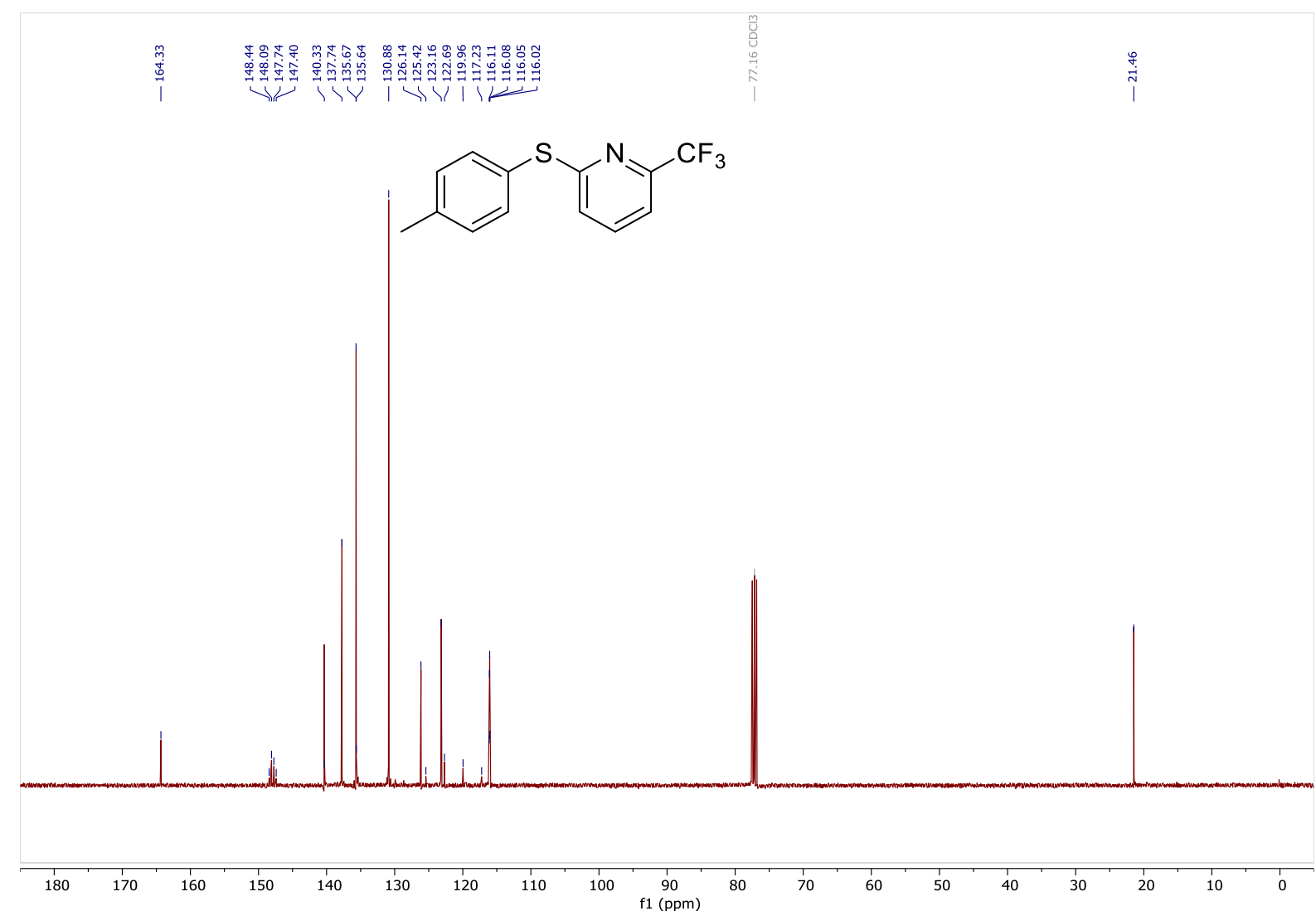


${ }^{1} \mathrm{H}$ NMR (400 MHz, $\mathrm{CDCl}_{3}$ ): 1ji

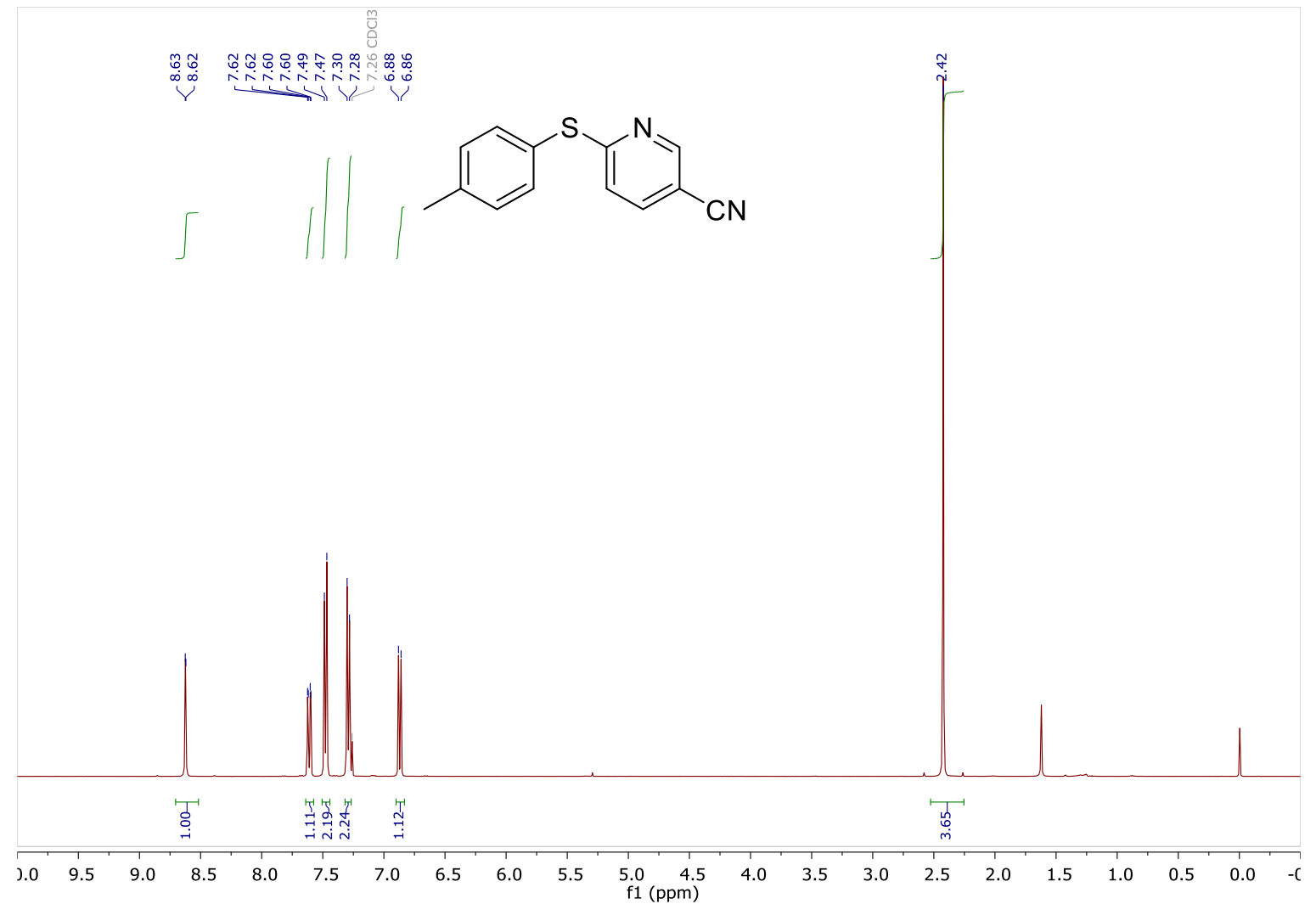

${ }^{13} \mathrm{C}$ NMR (101 MHz, $\left.\mathrm{CDCl}_{3}\right):$ 1ji

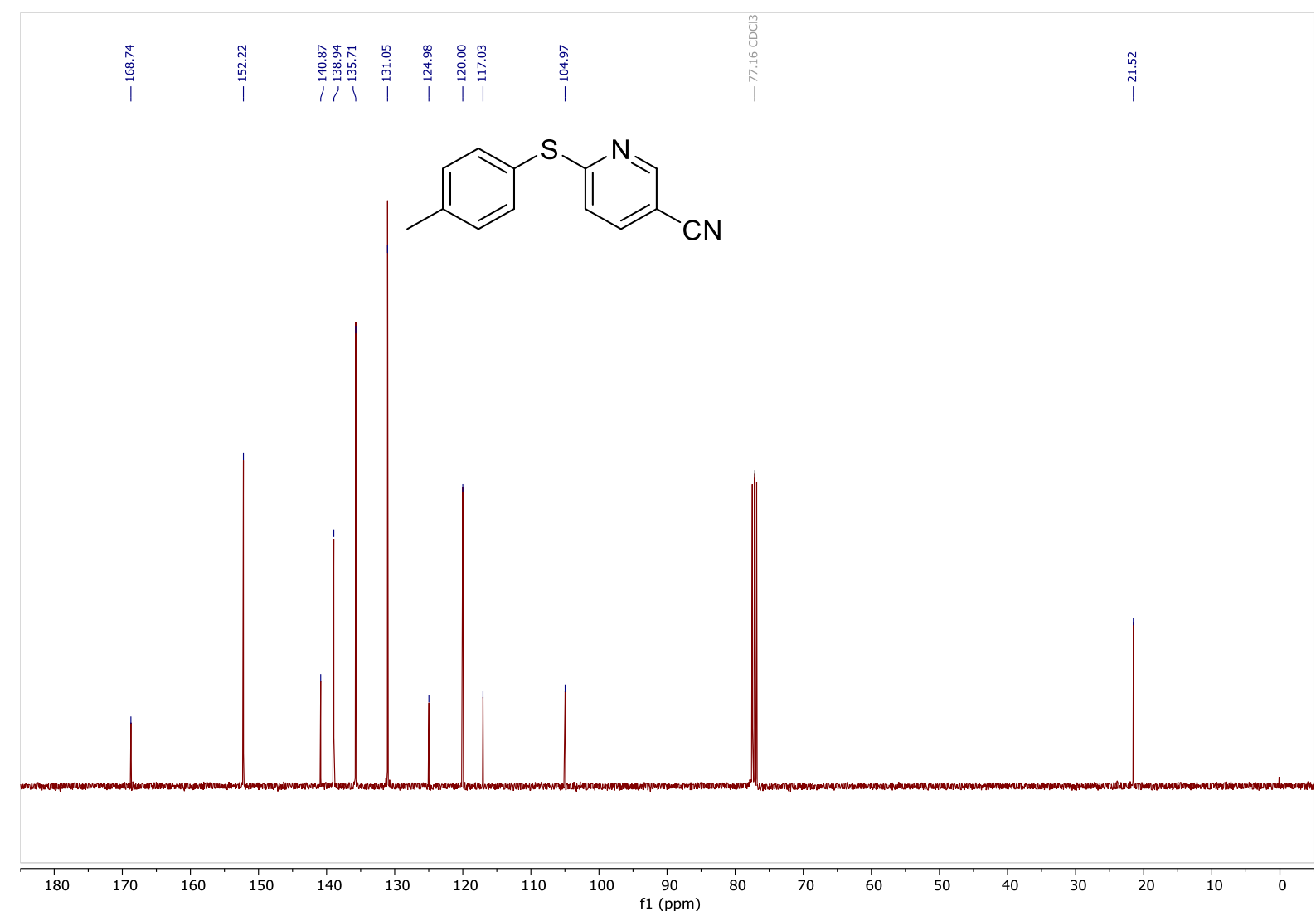


${ }^{1} \mathrm{H}$ NMR (400 MHz, $\left.\mathrm{CDCl}_{3}\right)$ : 1ki

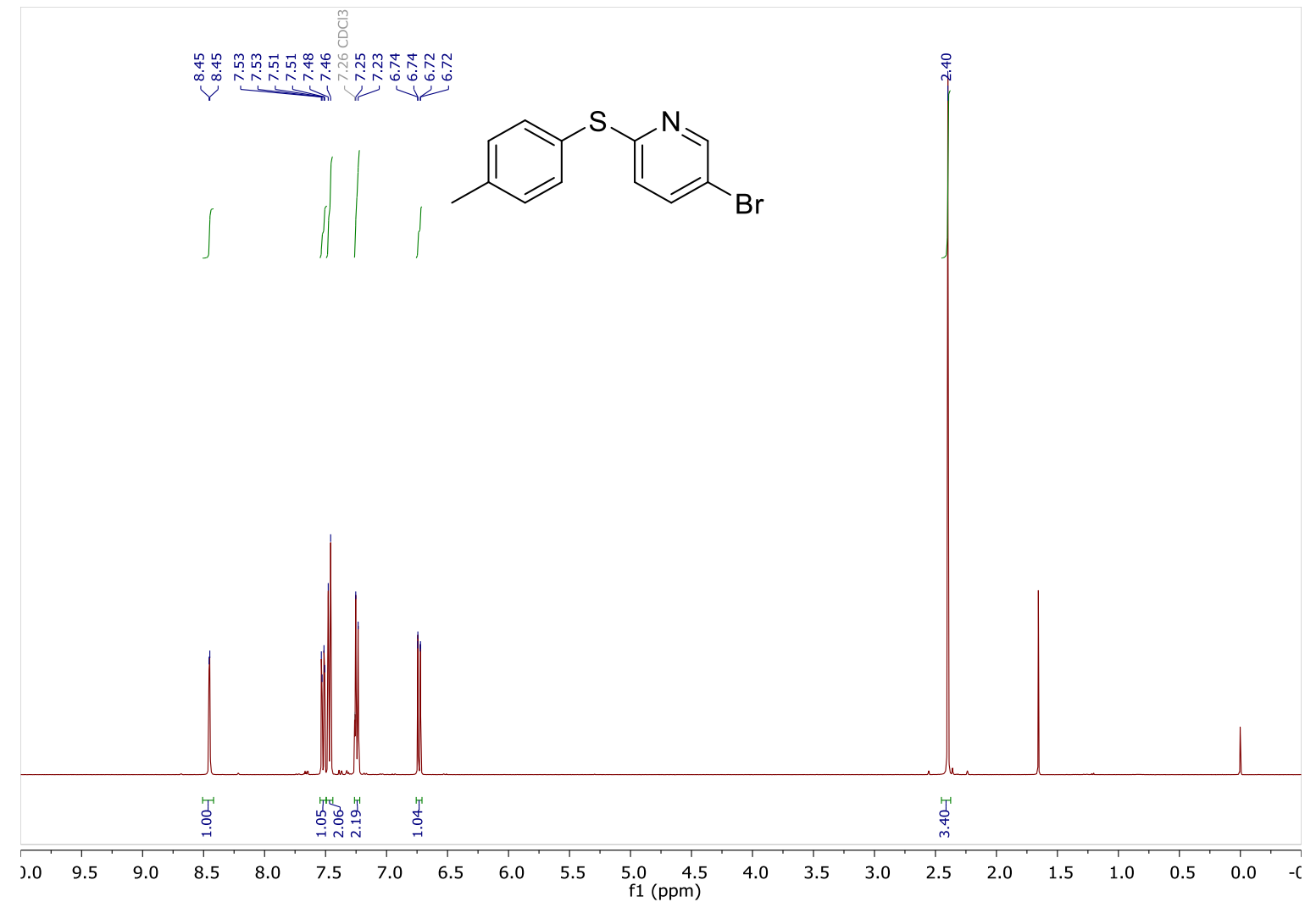

${ }^{13} \mathrm{C}$ NMR (101 MHz, $\left.\mathrm{CDCl}_{3}\right): 1 \mathrm{ki}$

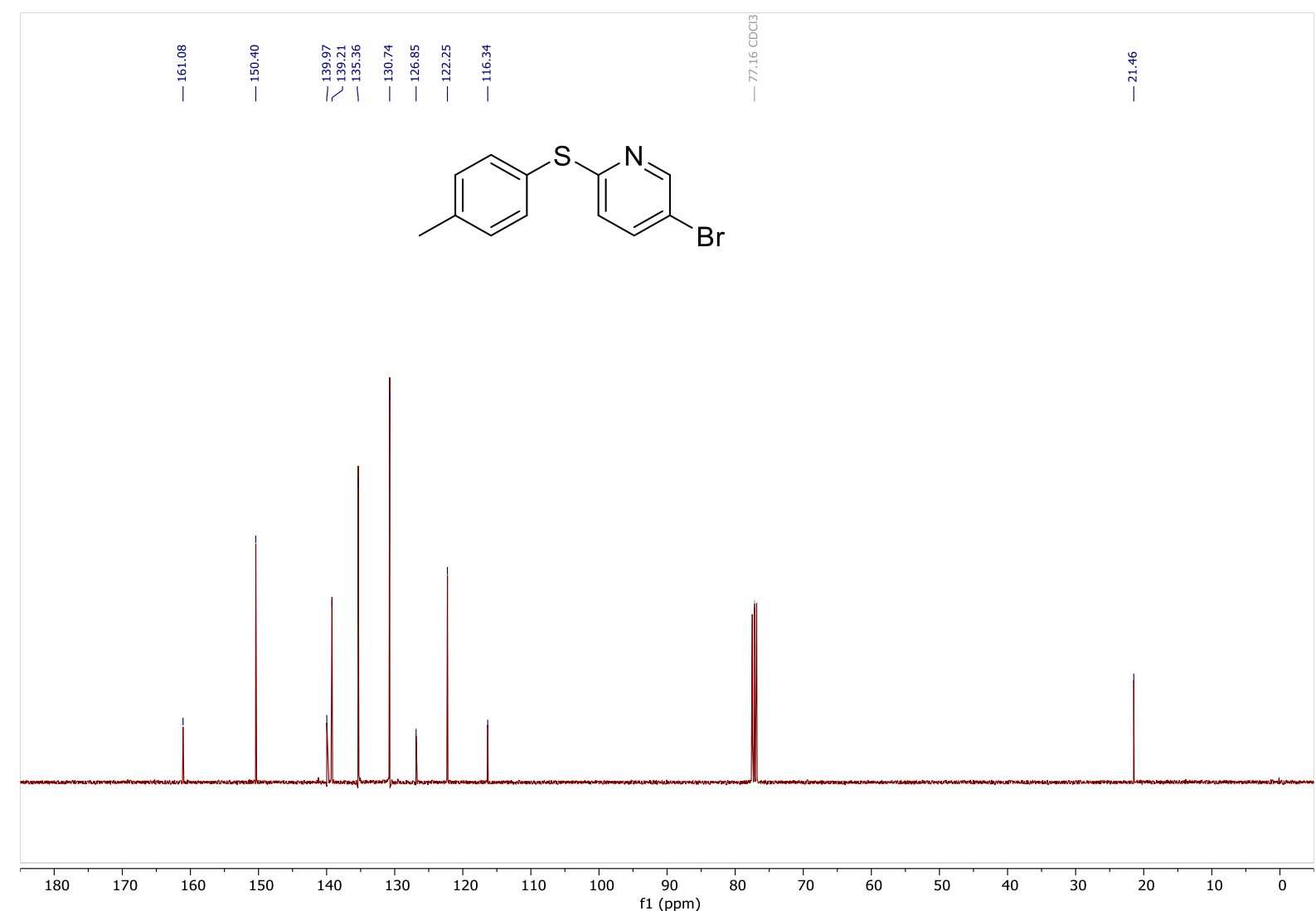


${ }^{1} \mathrm{H}$ NMR (400 MHz, $\mathrm{CDCl}_{3}$ ): $1 \mathrm{li}$

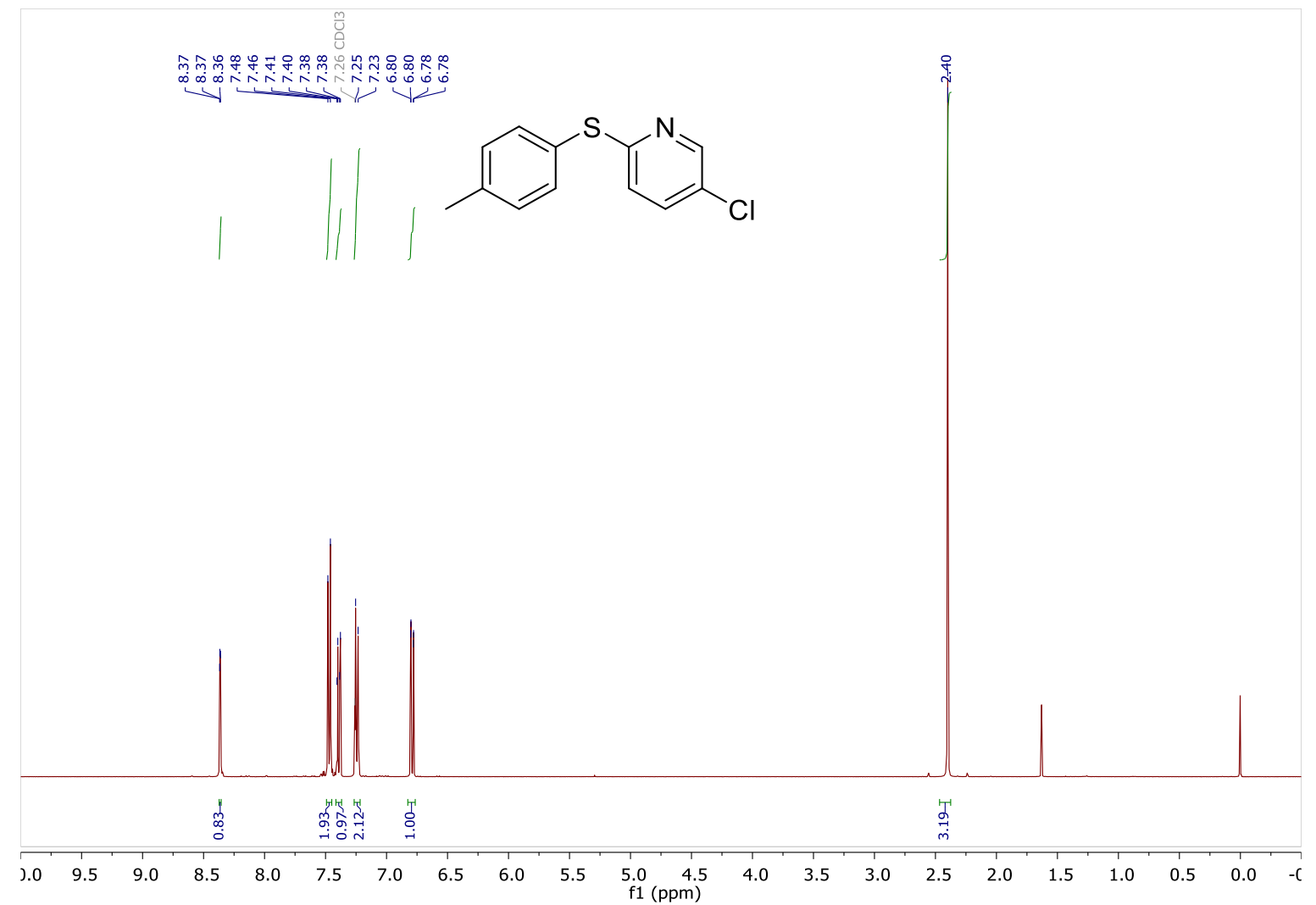

${ }^{13} \mathrm{C}$ NMR (101 MHz, $\left.\mathrm{CDCl}_{3}\right): 1 \mathrm{li}$

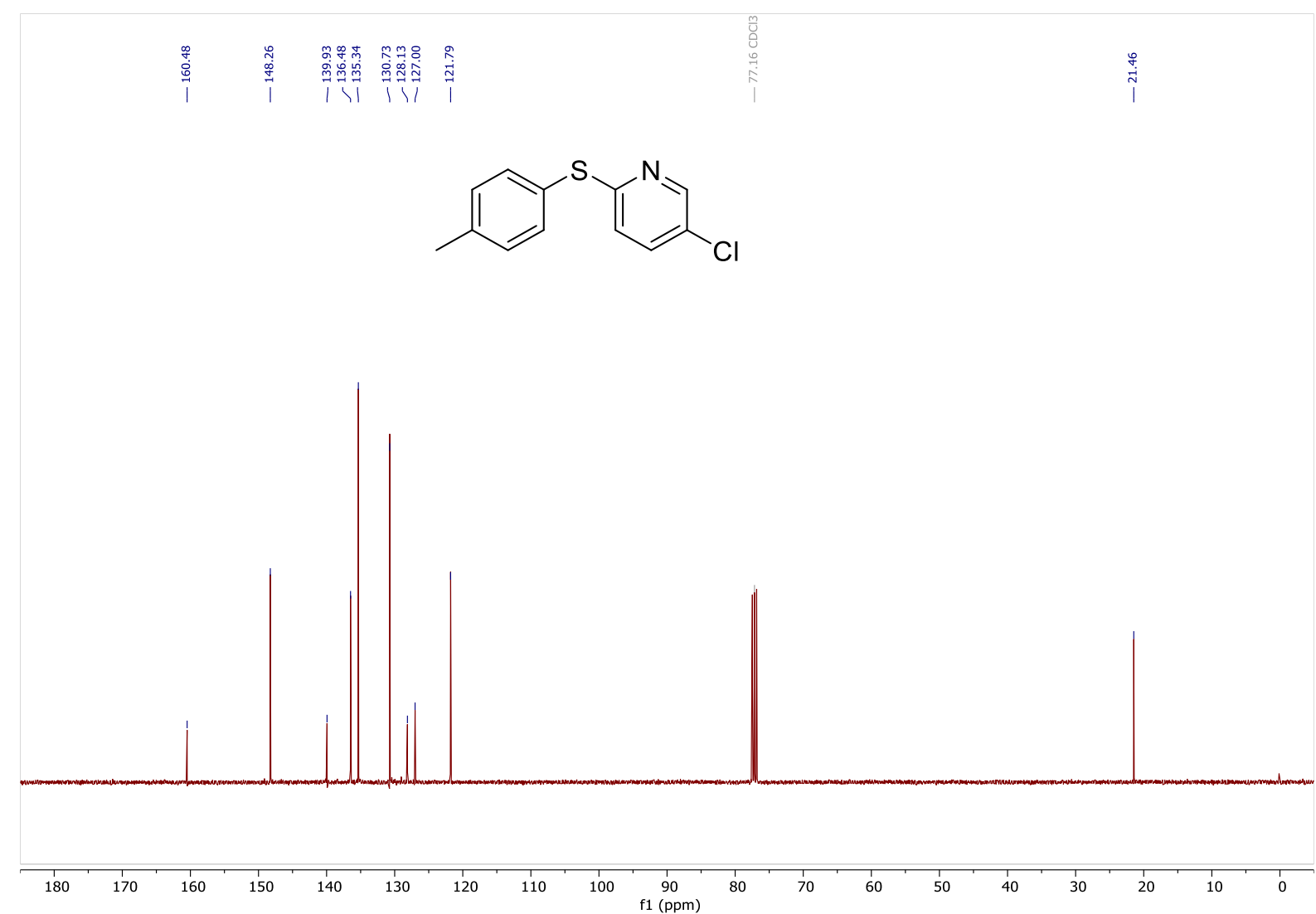


${ }^{1} \mathrm{H}$ NMR (400 MHz, $\mathrm{CDCl}_{3}$ ): 1 $\mathrm{mi}$

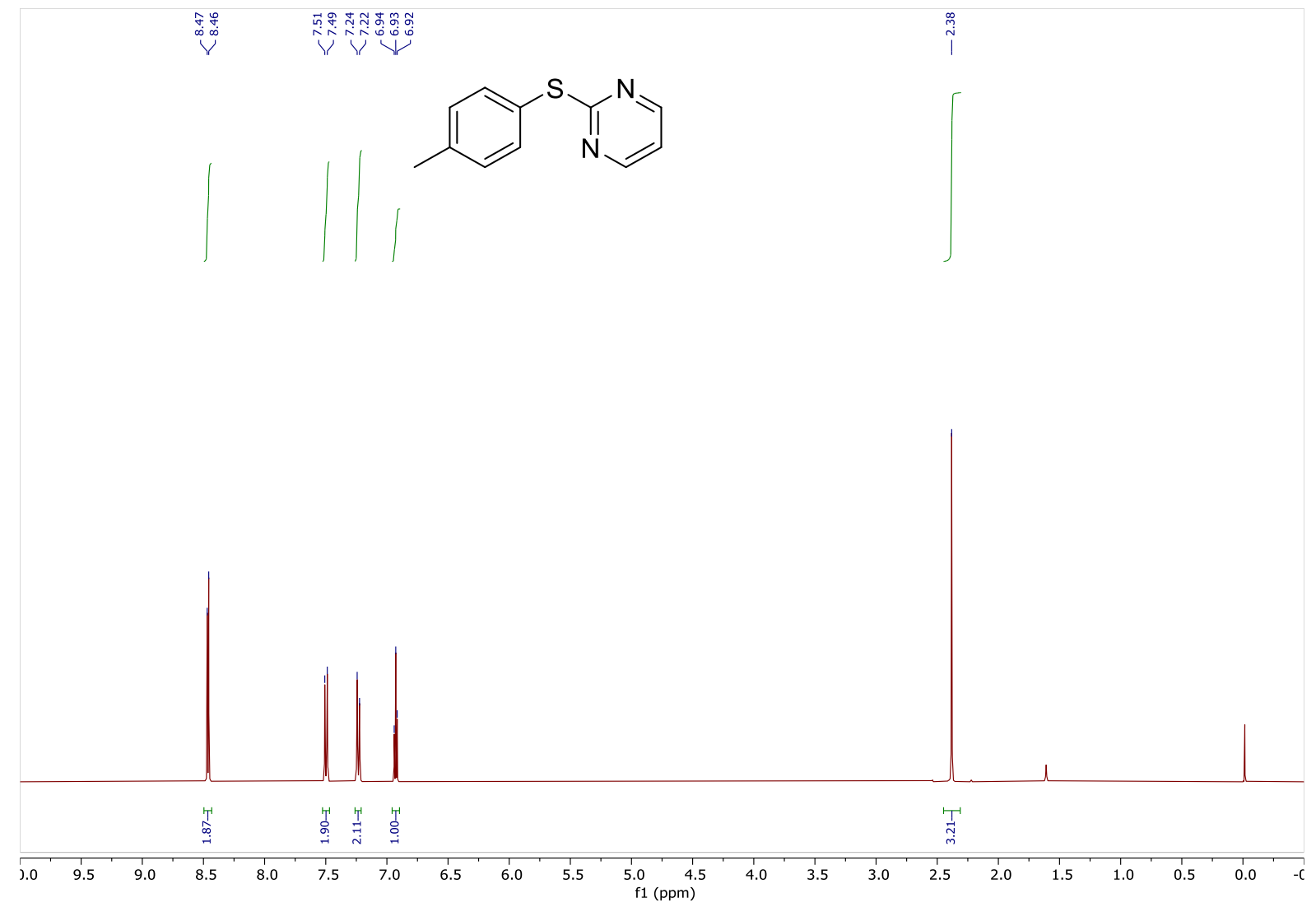

${ }^{13} \mathrm{C}$ NMR (101 MHz, $\left.\mathrm{CDCl}_{3}\right): 1 \mathrm{mi}$

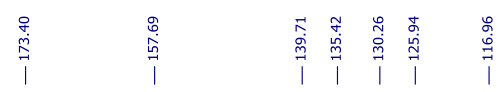<smiles>Cc1ccc(Sc2ncccn2)cc1</smiles>

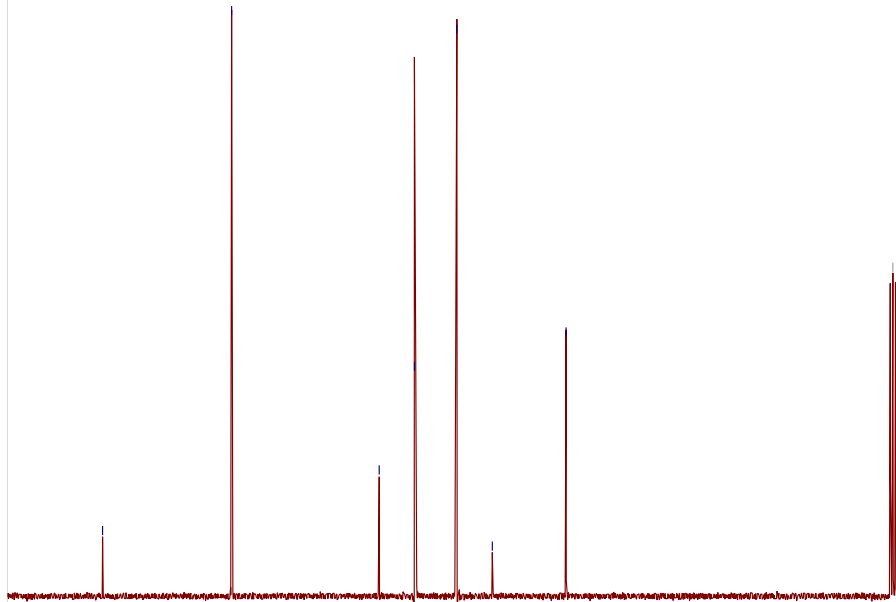

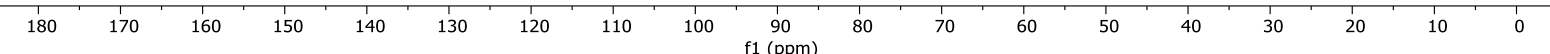


${ }^{1} \mathrm{H}$ NMR (500 MHz, $\mathrm{CDCl}_{3}$ ): $1 \mathrm{~h}$

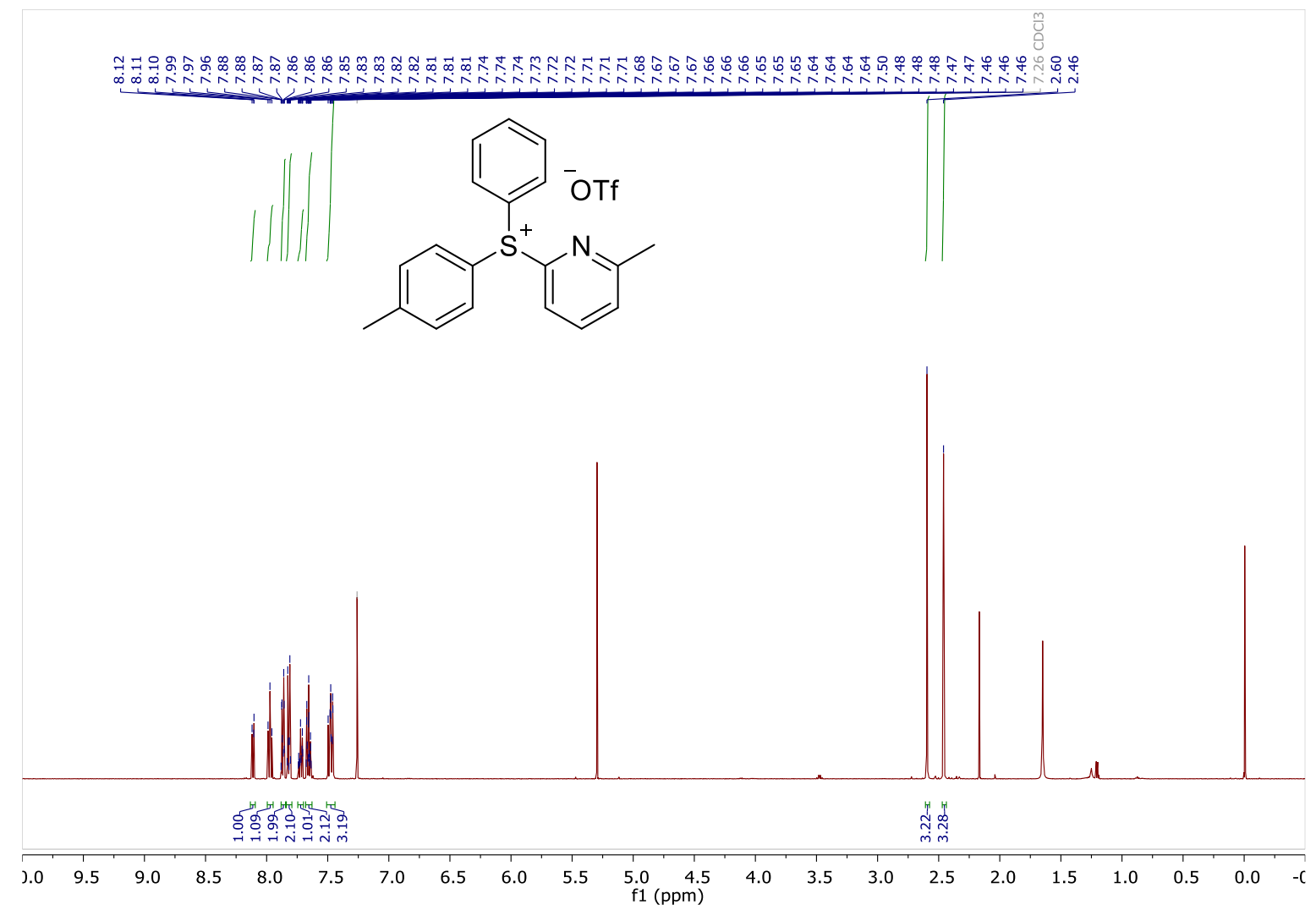

${ }^{13} \mathrm{C}$ NMR (126 MHz, $\left.\mathrm{CDCl}_{3}\right): 1 \mathrm{~h}$

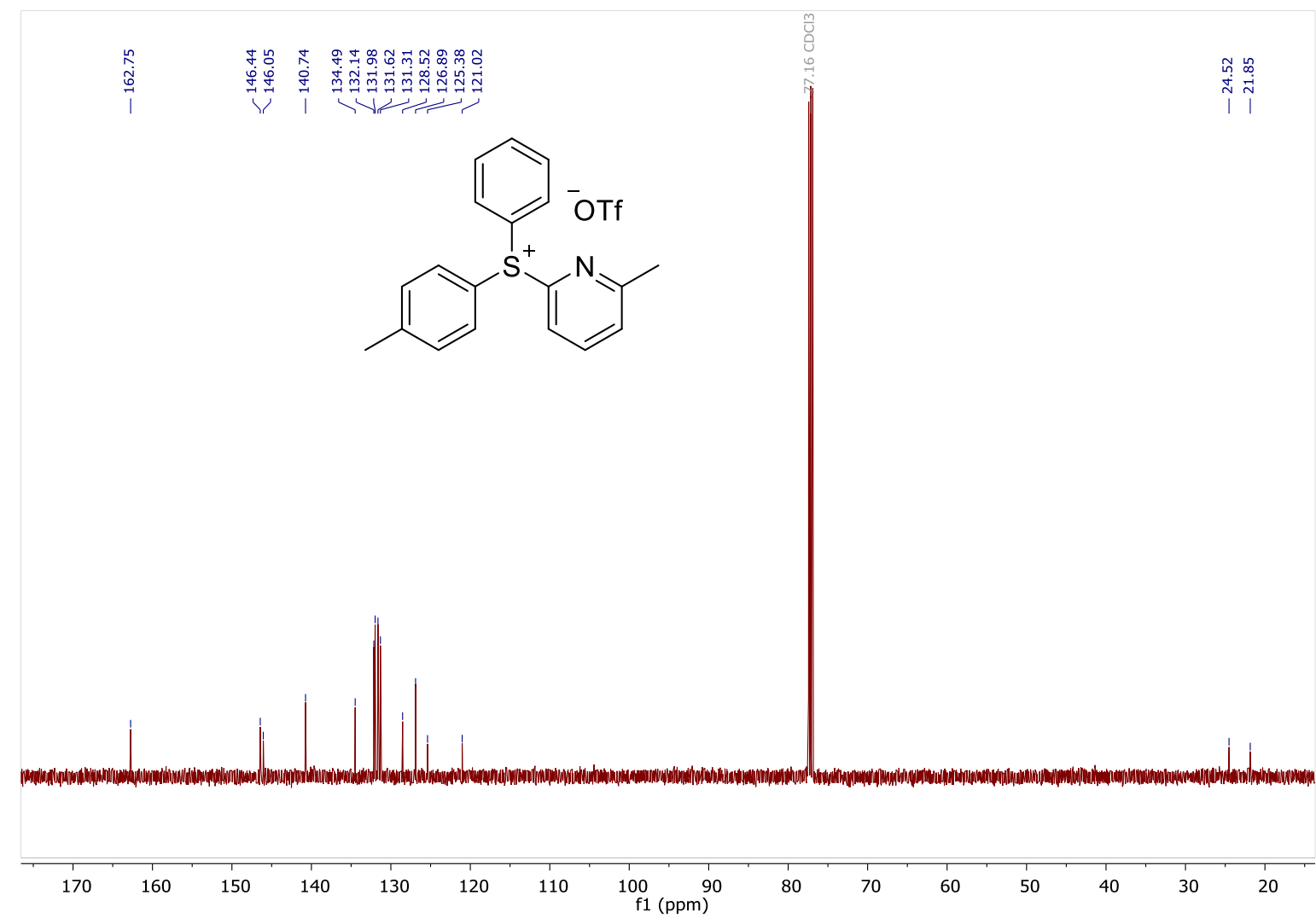


${ }^{1} \mathrm{H}$ NMR (400 MHz, $\mathrm{CDCl}_{3}$ ): 1i

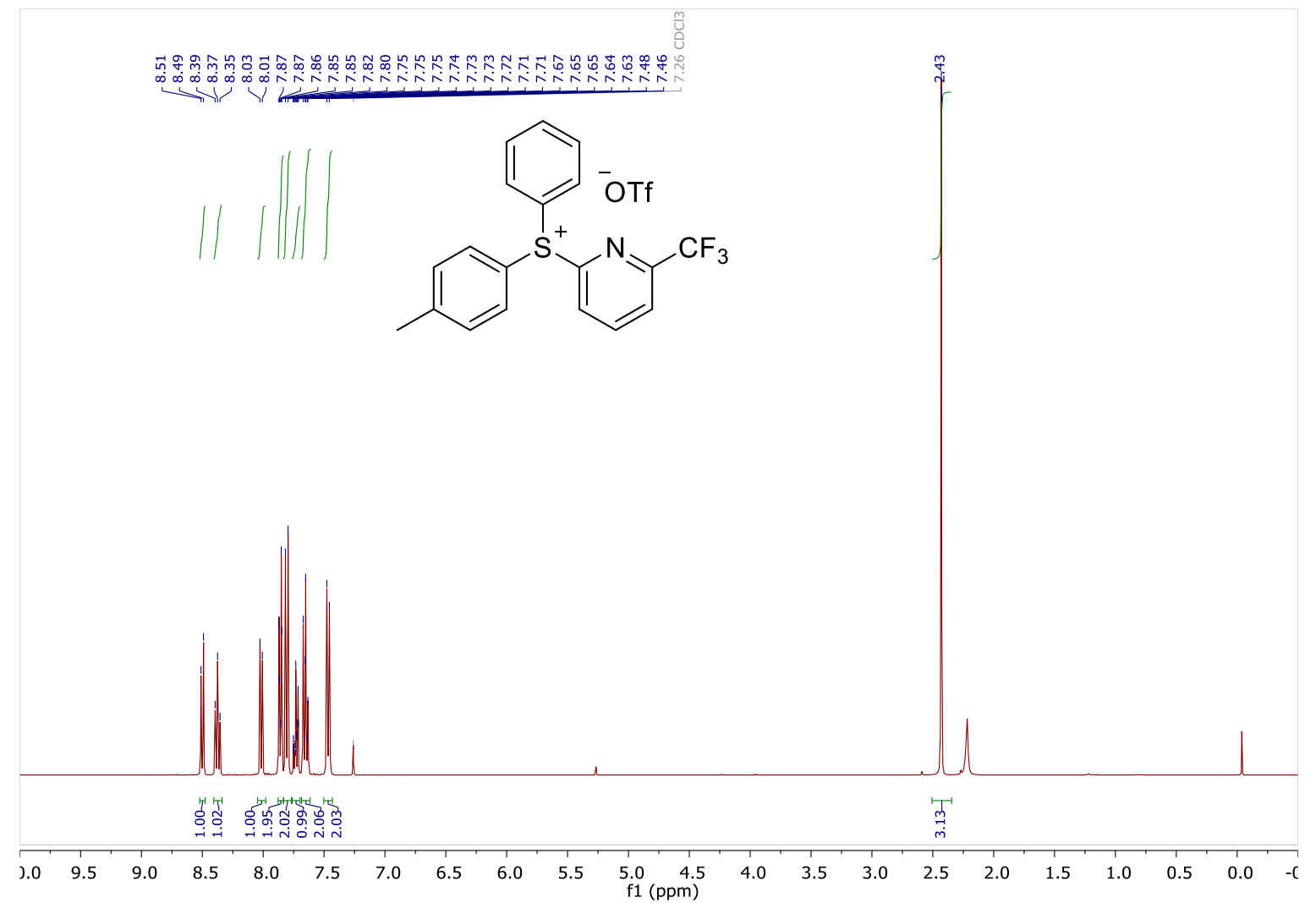

${ }^{13} \mathrm{C}$ NMR (101 MHz, $\left.\mathrm{CDCl}_{3}\right): 1 \mathrm{i}$

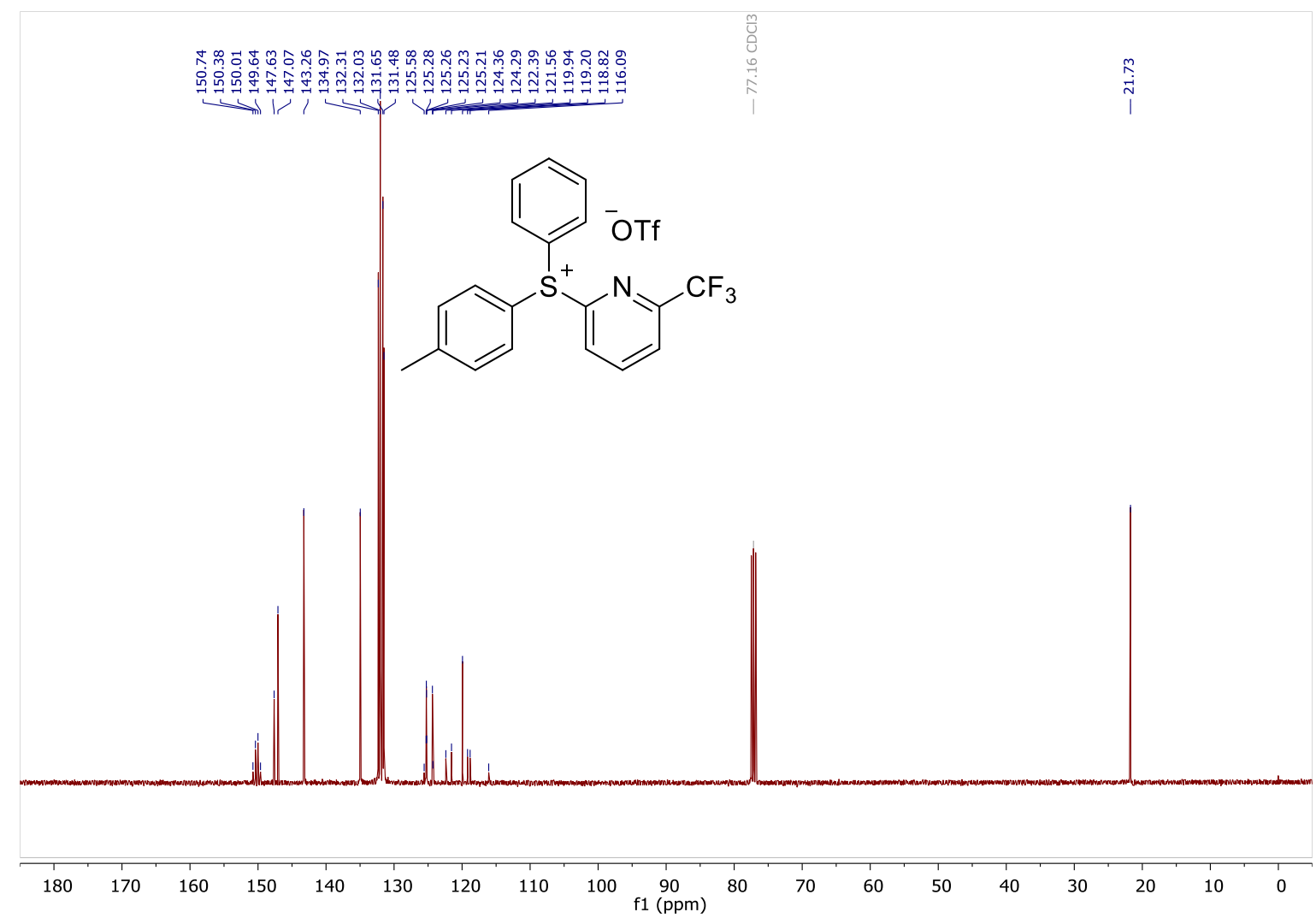


${ }^{1} \mathrm{H}$ NMR (500 MHz, $\left.\mathrm{CDCl}_{3}\right): 1 \mathbf{j}$

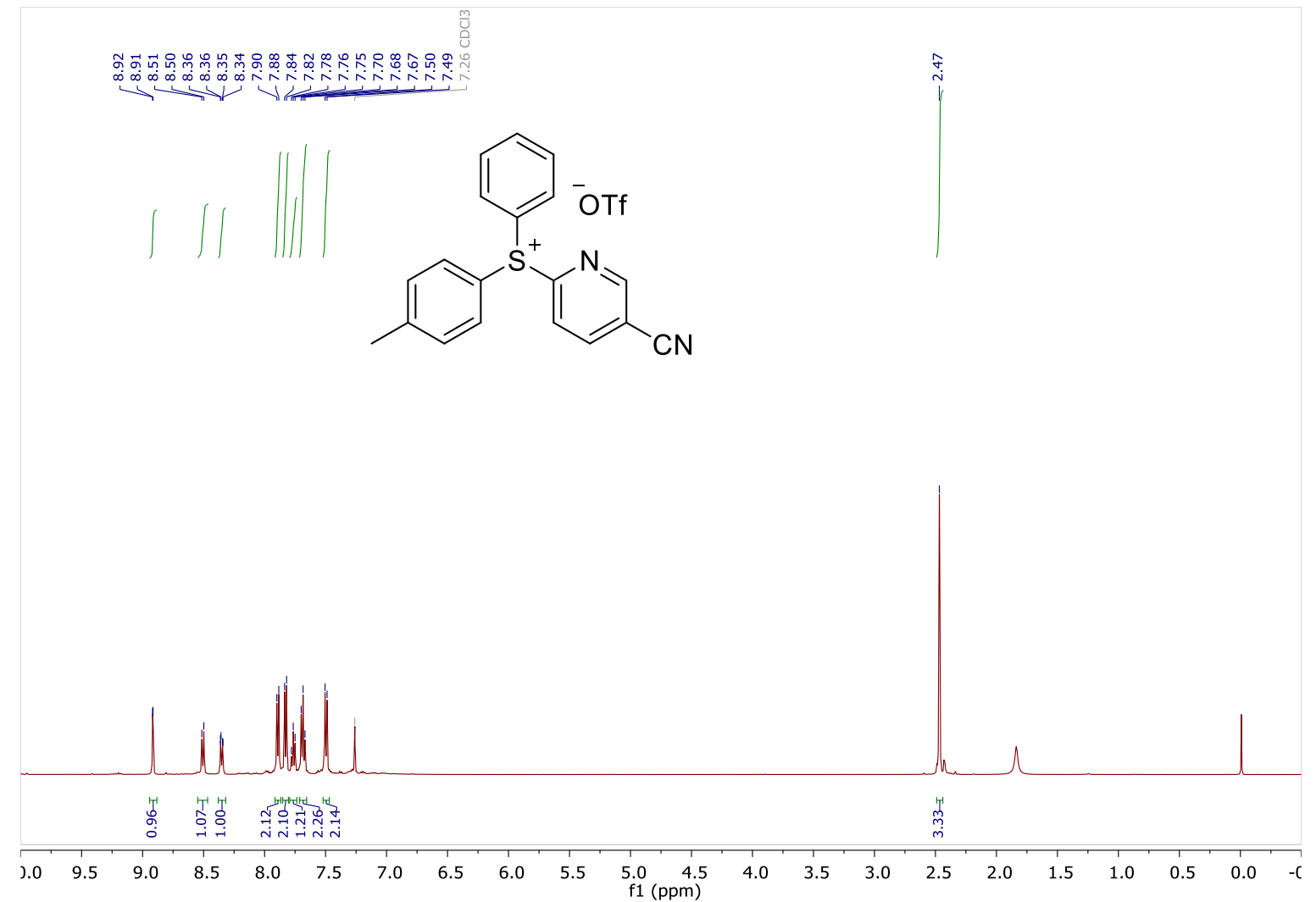

${ }^{13} \mathrm{C}$ NMR (126 MHz, $\left.\mathrm{CDCl}_{3}\right):$ 1j

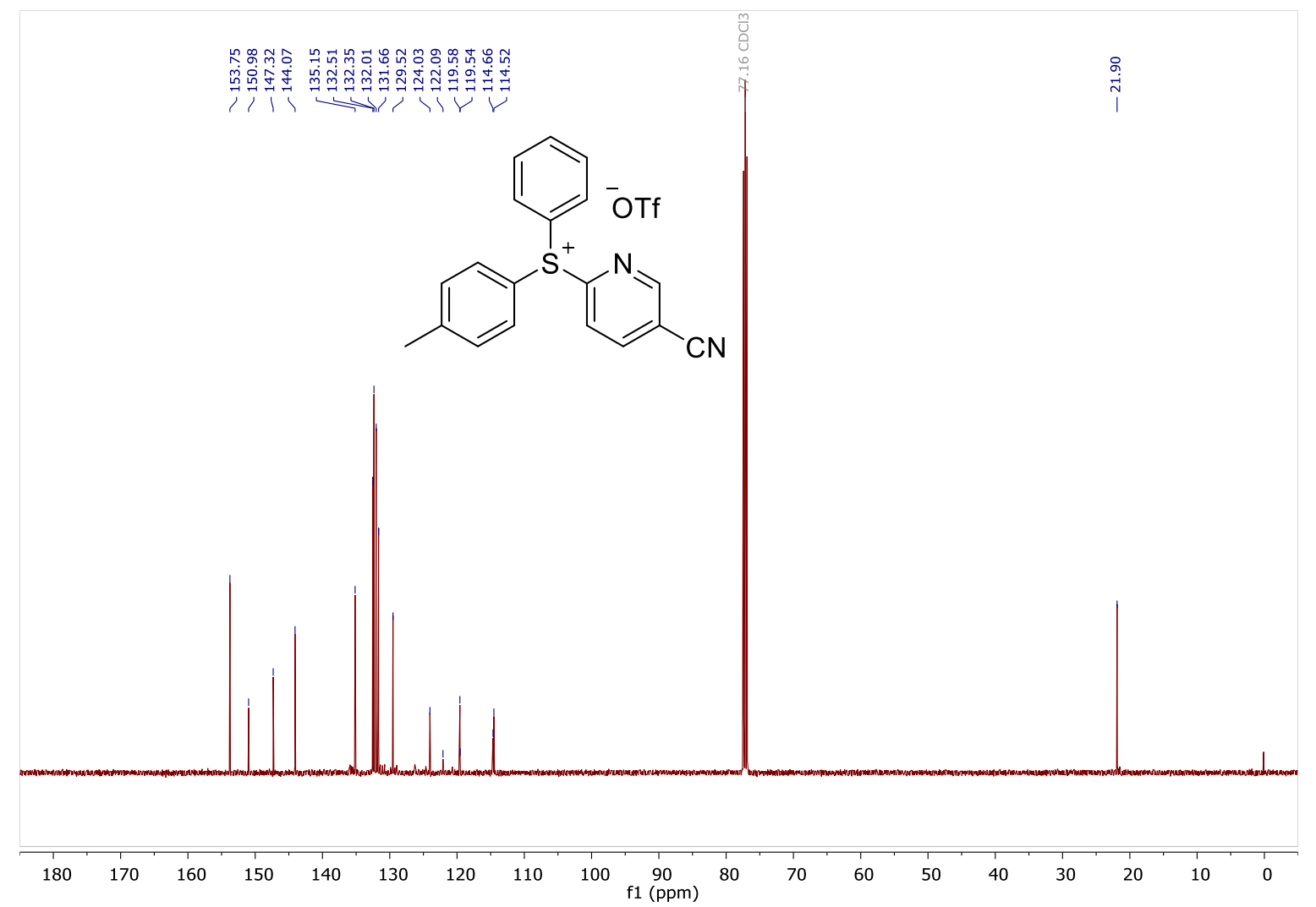


${ }^{1} \mathrm{H}$ NMR (500 MHz, $\left.\mathrm{CDCl}_{3}\right): 1 \mathrm{k}$

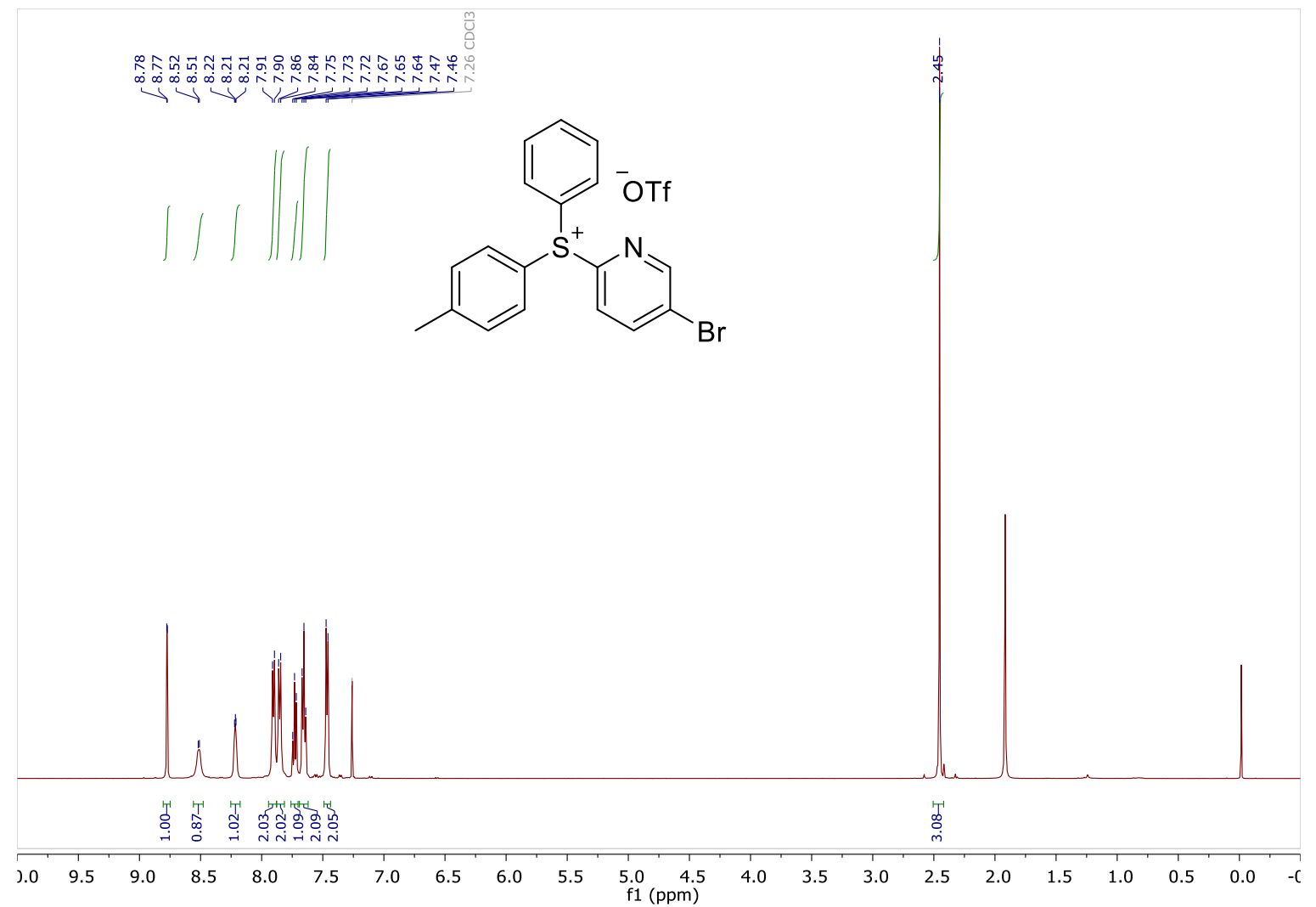

${ }^{13} \mathrm{C}$ NMR (126 MHz, $\left.\mathrm{CDCl}_{3}\right): 1 \mathrm{k}$

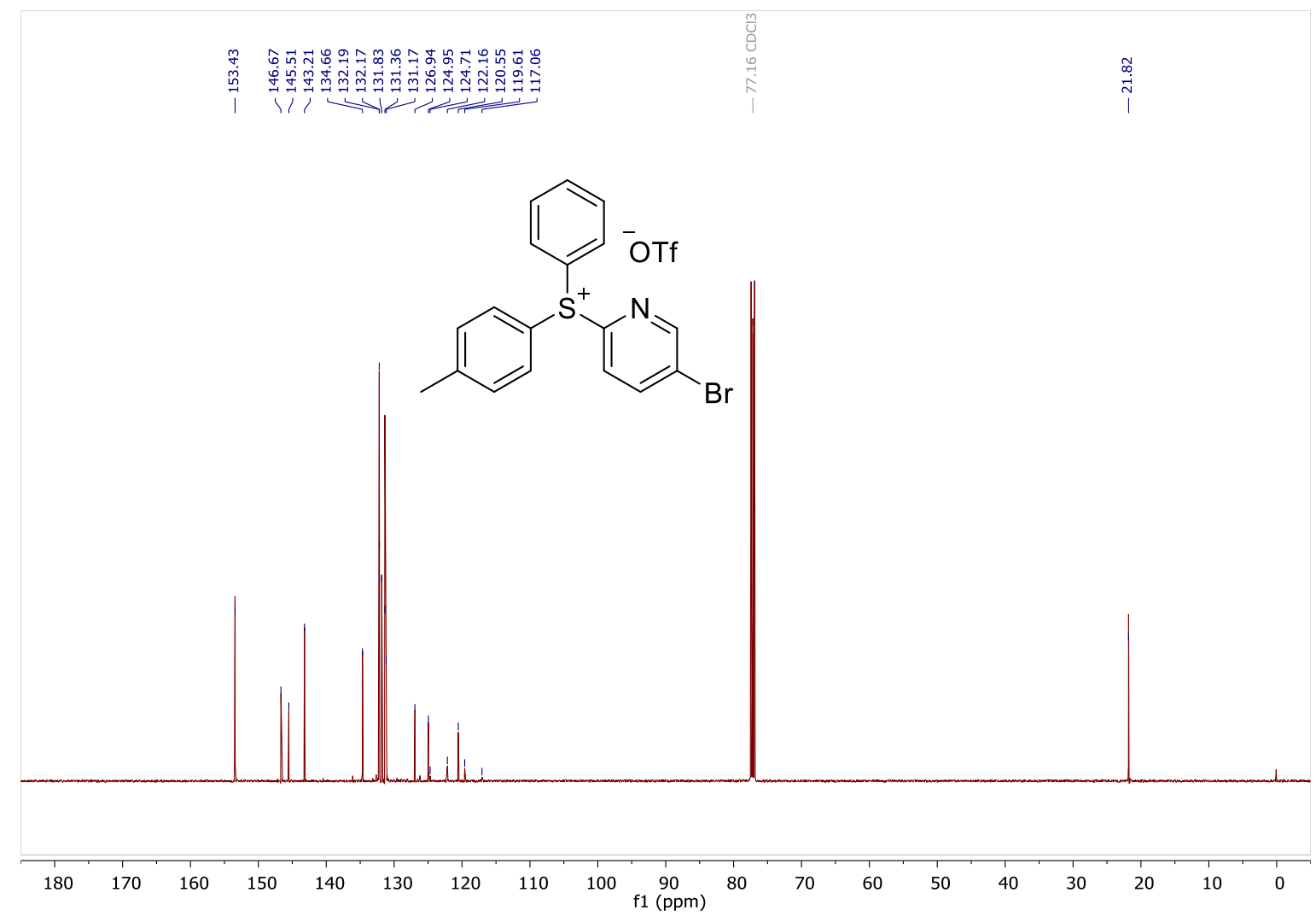


${ }^{1} \mathrm{H}$ NMR (500 MHz, $\mathrm{CDCl}_{3}$ ): 11

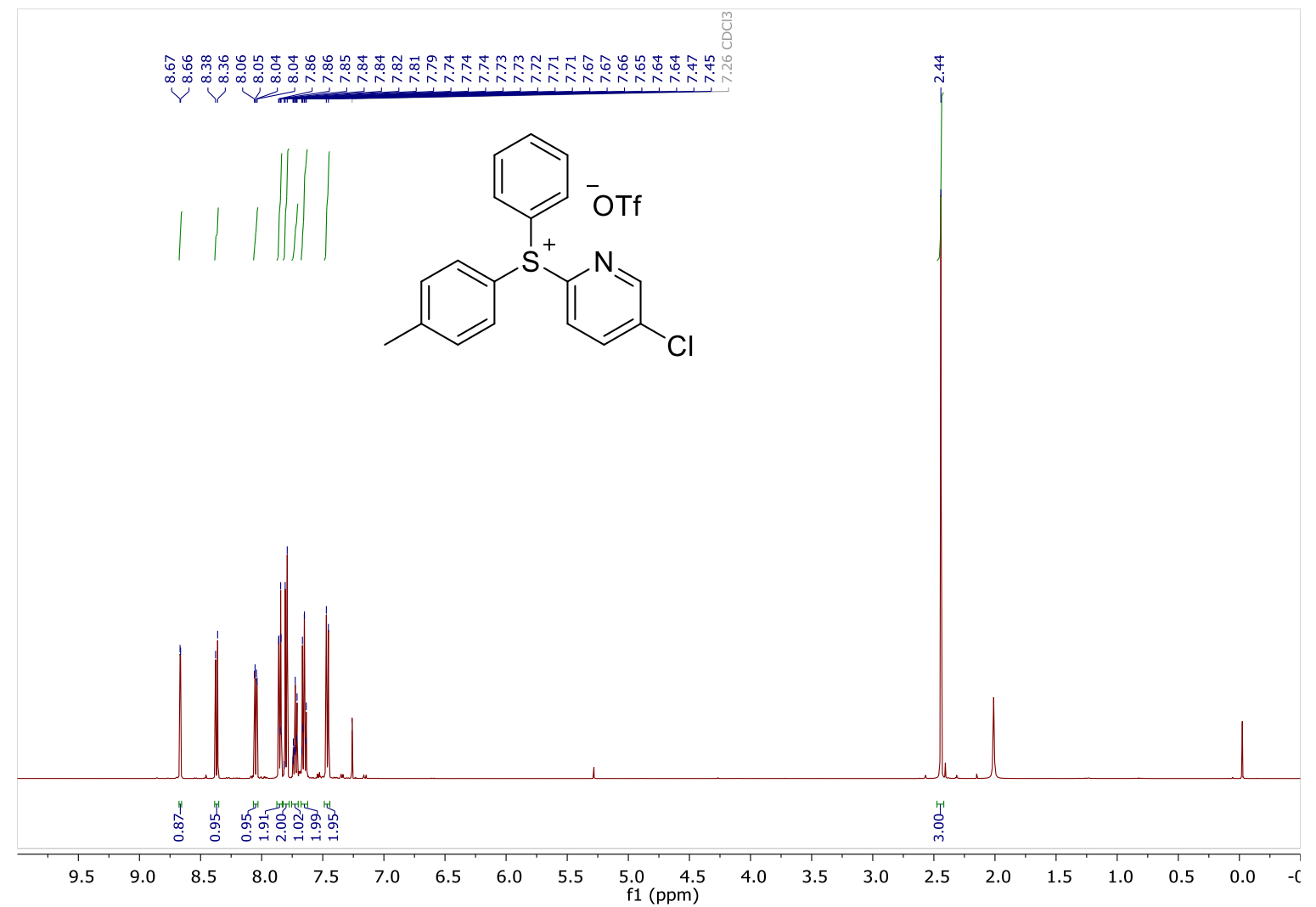

${ }^{13} \mathrm{C}$ NMR (101 MHz, $\left.\mathrm{CDCl}_{3}\right): 11$

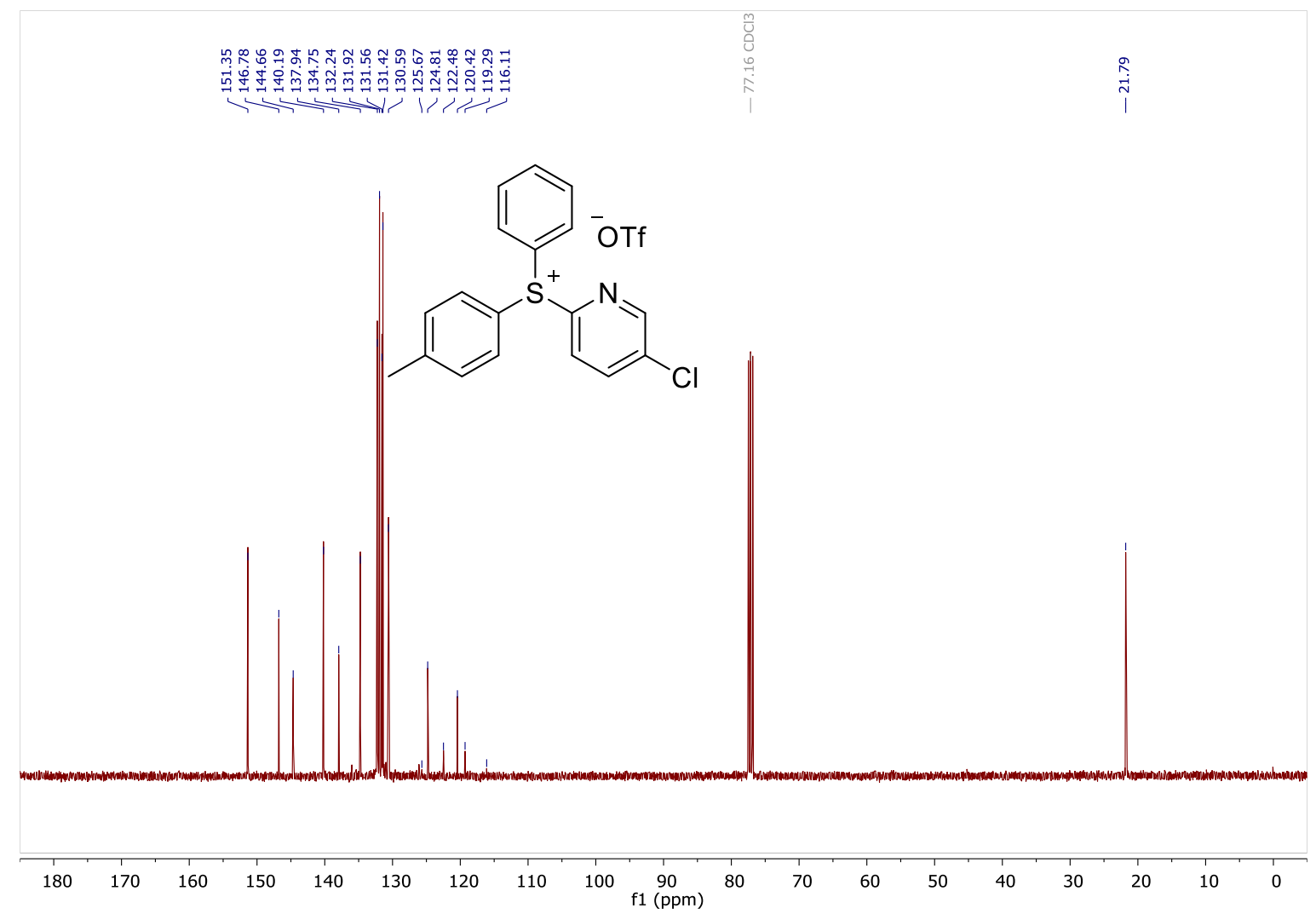


${ }^{1} \mathrm{H}$ NMR (500 MHz, $\left.\mathrm{CDCl}_{3}\right): 1 \mathrm{~m}$

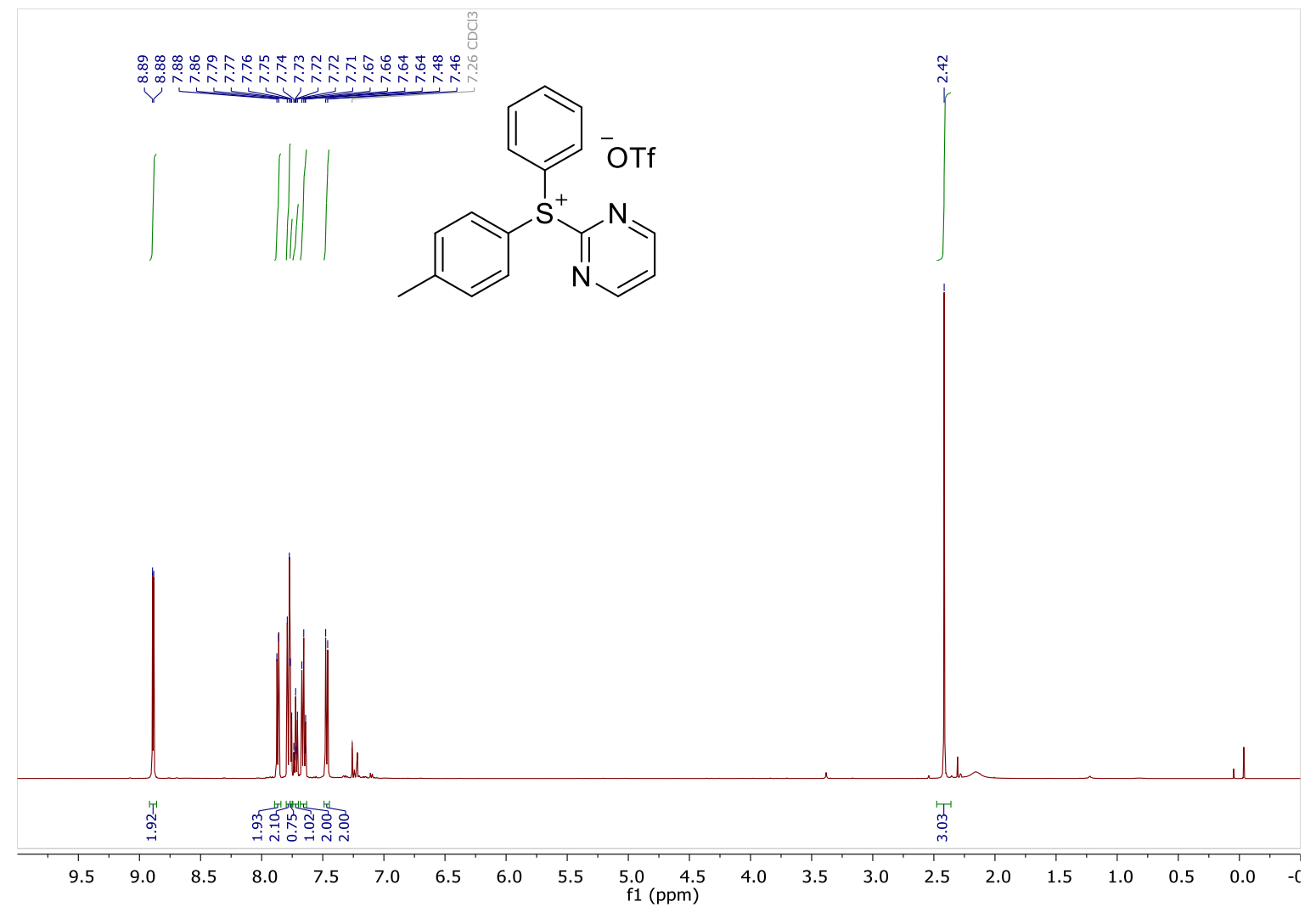

${ }^{13} \mathrm{C}$ NMR (126 MHz, $\left.\mathrm{CDCl}_{3}\right): 1 \mathrm{~m}$

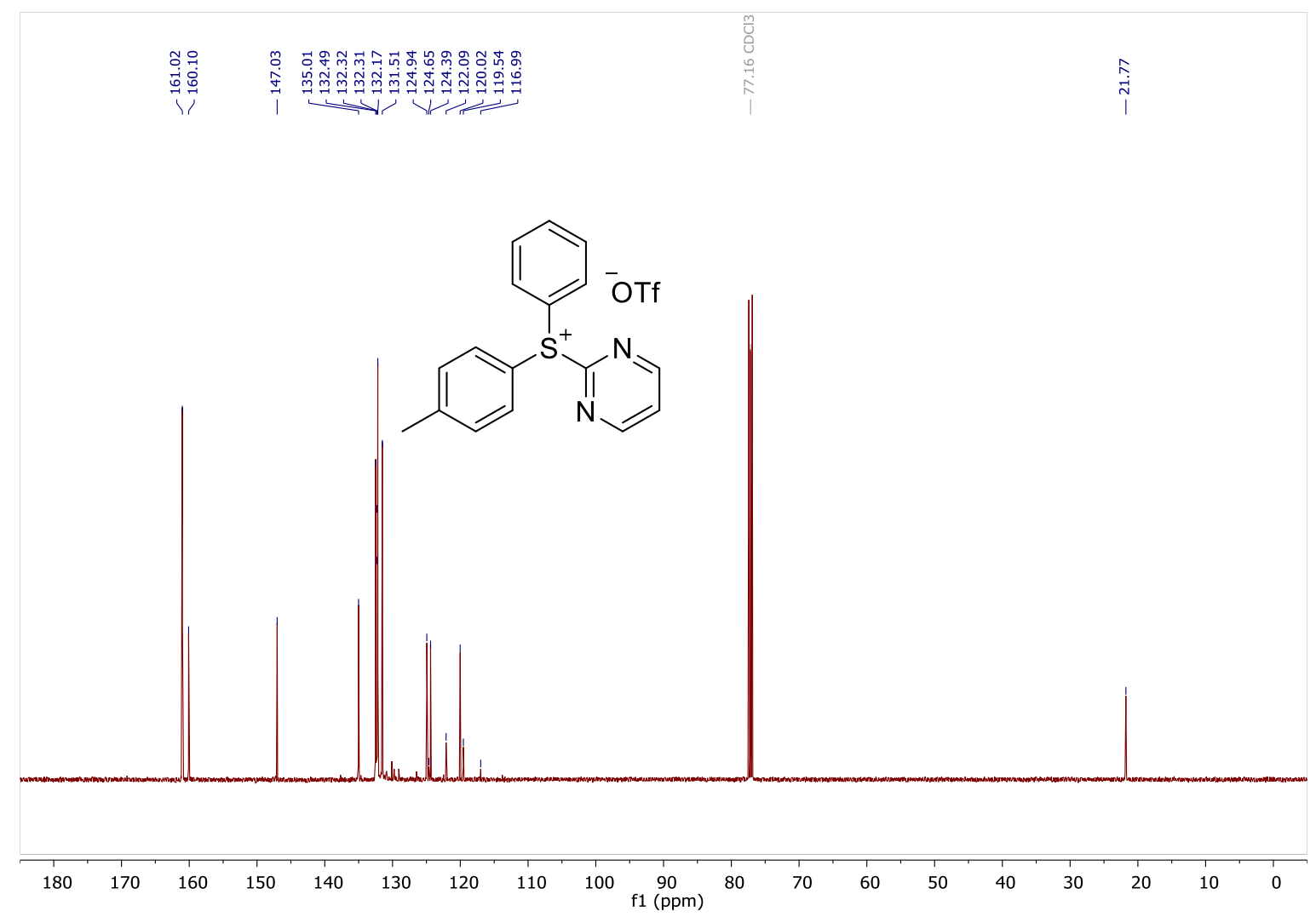


${ }^{1} \mathrm{H}$ NMR (400 MHz, $\left.\mathrm{CDCl}_{3}\right): 2$

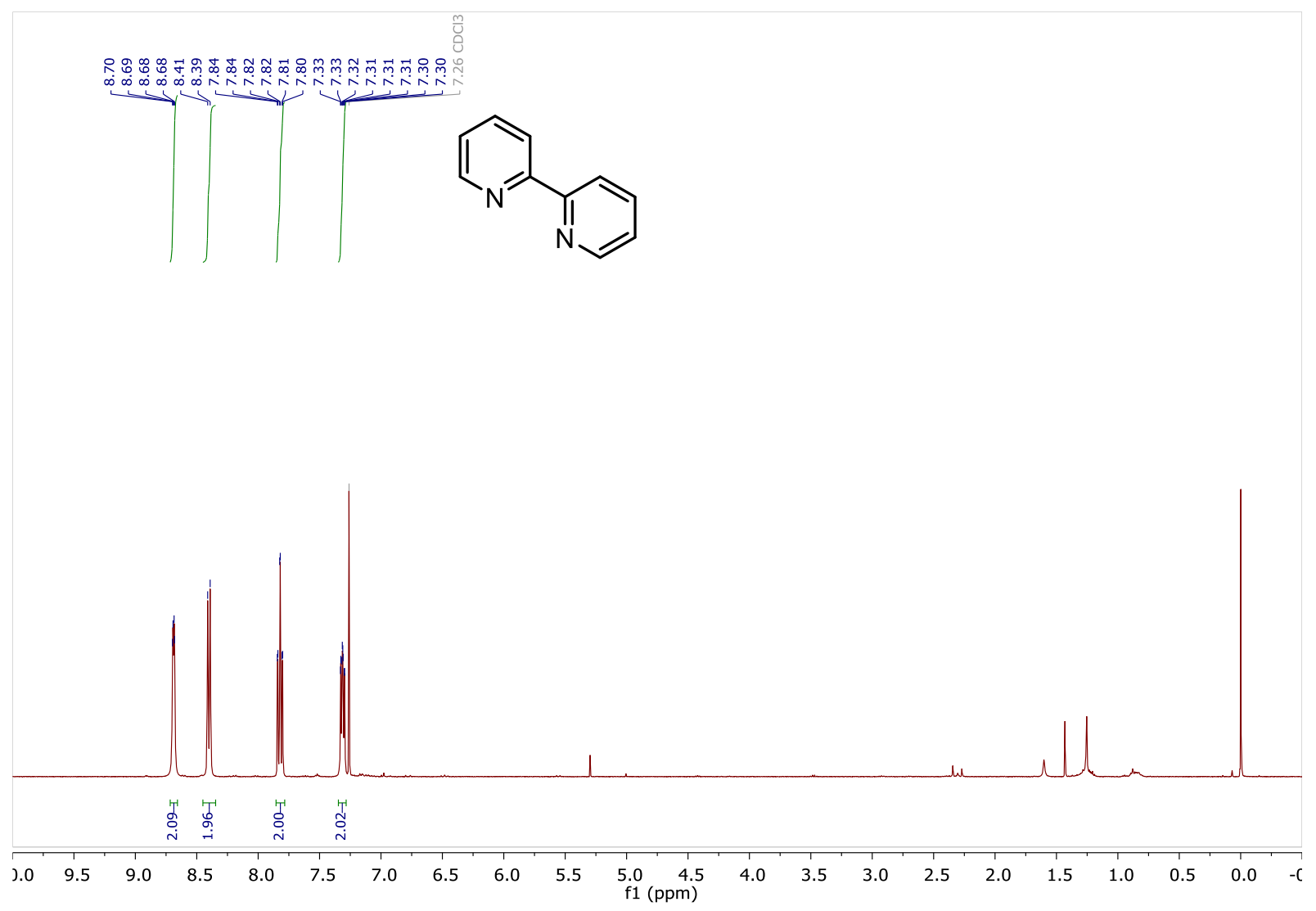

${ }^{13} \mathrm{C}$ NMR (101 MHz, $\left.\mathrm{CDCl}_{3}\right): 2$

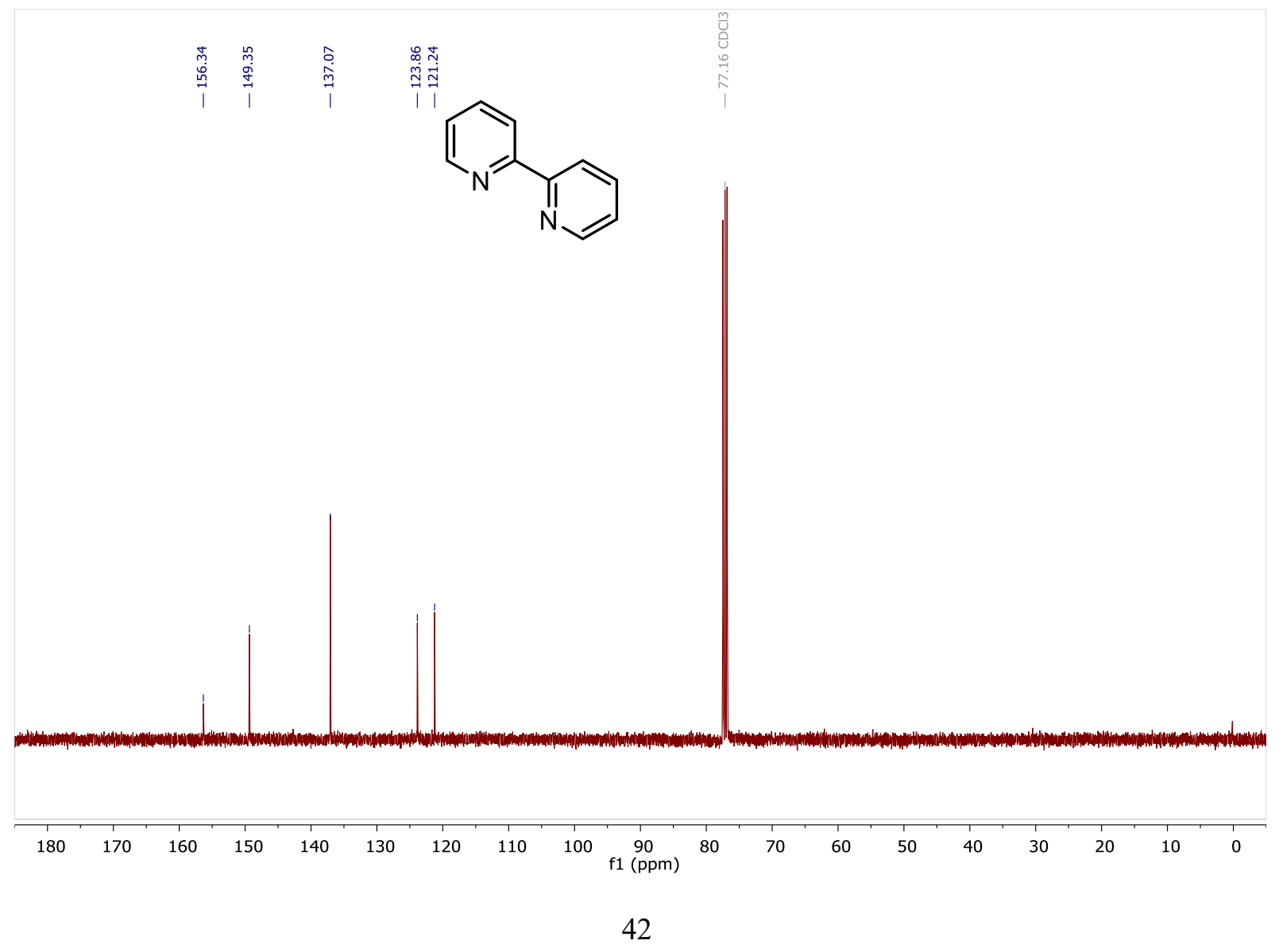


${ }^{1} \mathrm{H}$ NMR (400 MHz, $\mathrm{CDCl}_{3}$ ): 3

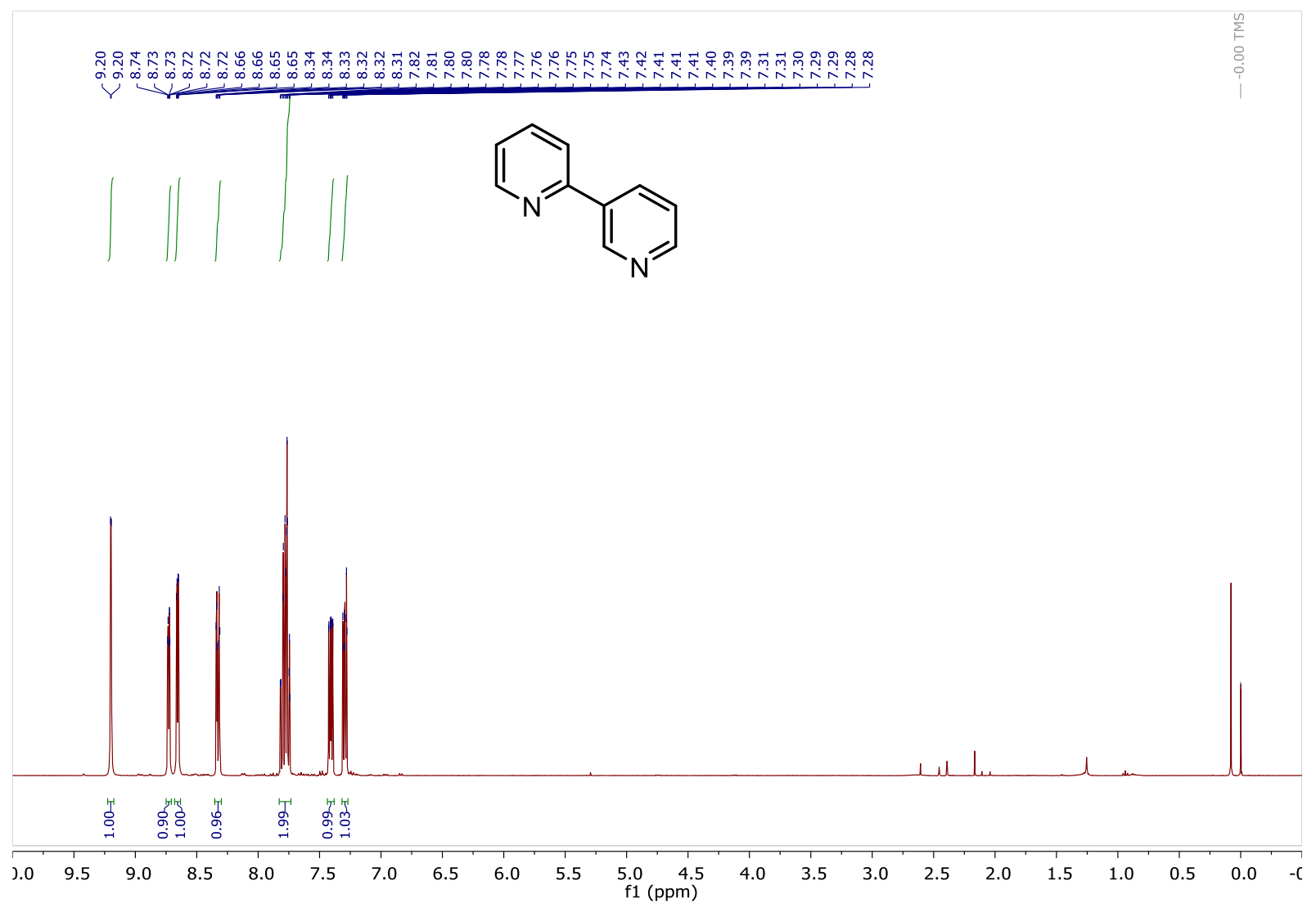

${ }^{13} \mathrm{C}$ NMR (101 MHz, $\left.\mathrm{CDCl}_{3}\right): 3$

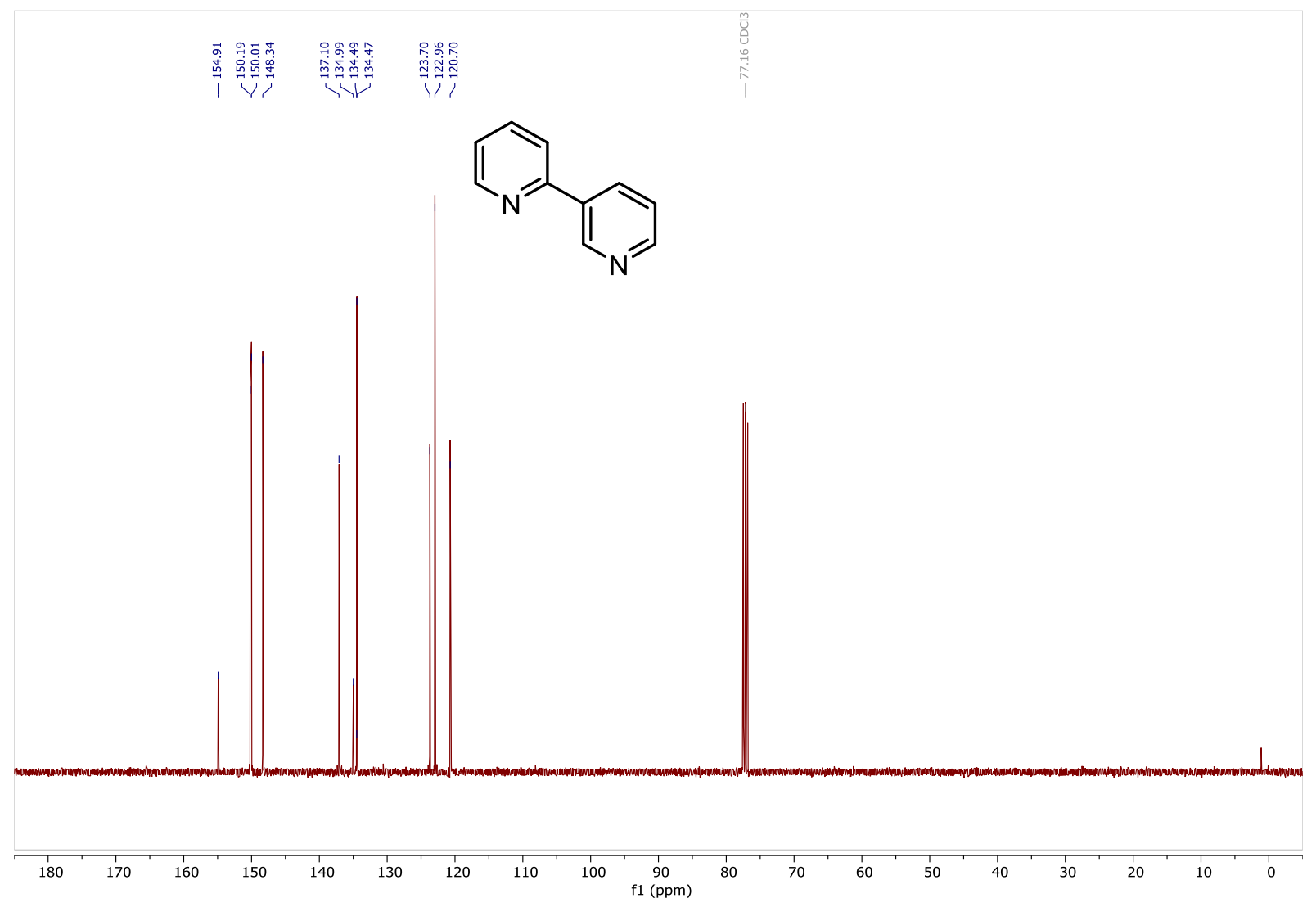

43 
${ }^{1} \mathrm{H}$ NMR (500 MHz, $\left.\mathrm{CDCl}_{3}\right): 4$

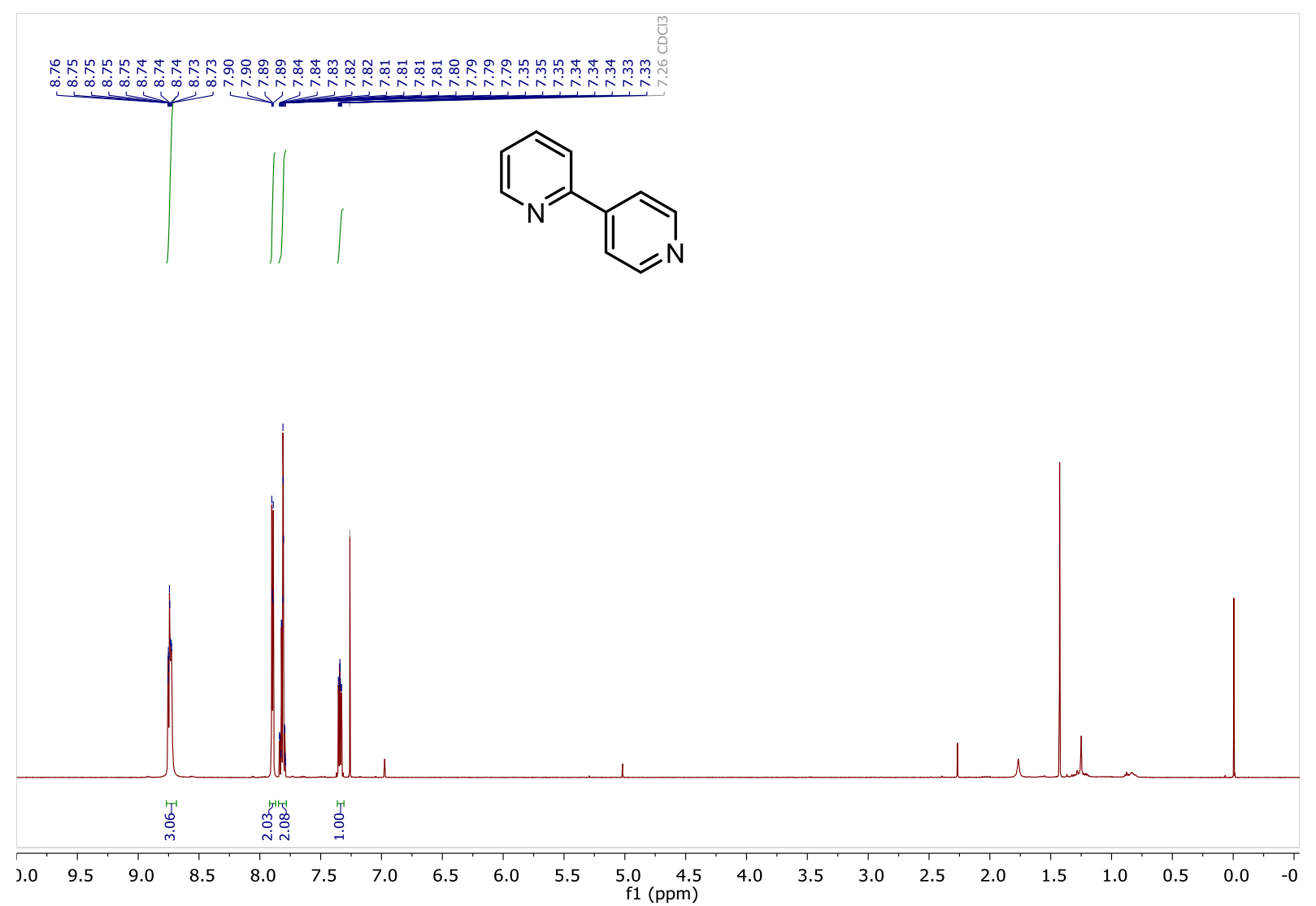

${ }^{13} \mathrm{C}$ NMR (126 MHz, $\left.\mathrm{CDCl}_{3}\right): 4$

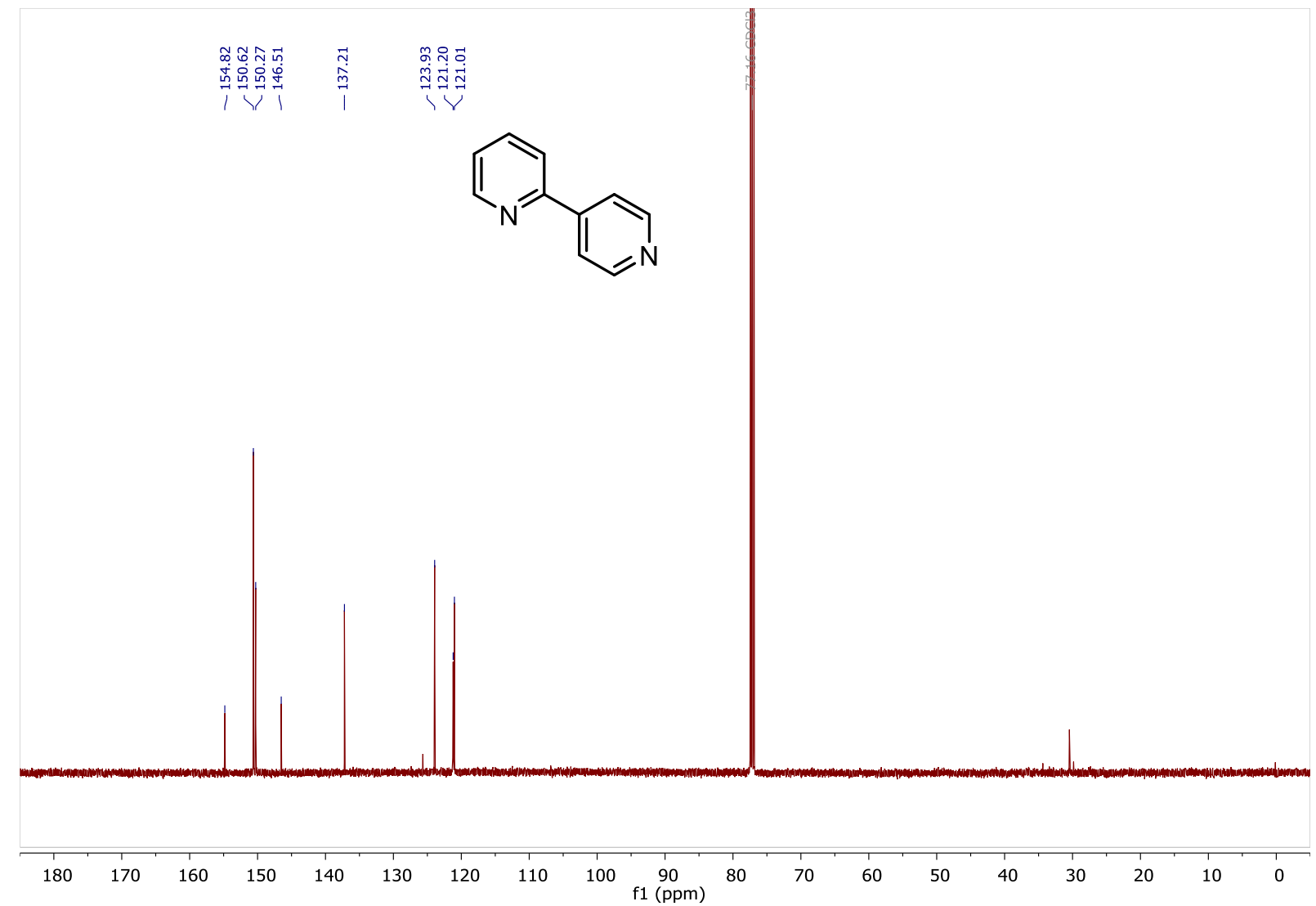


${ }^{1} \mathrm{H}$ NMR (500 MHz, $\left.\mathrm{CDCl}_{3}\right): 5$

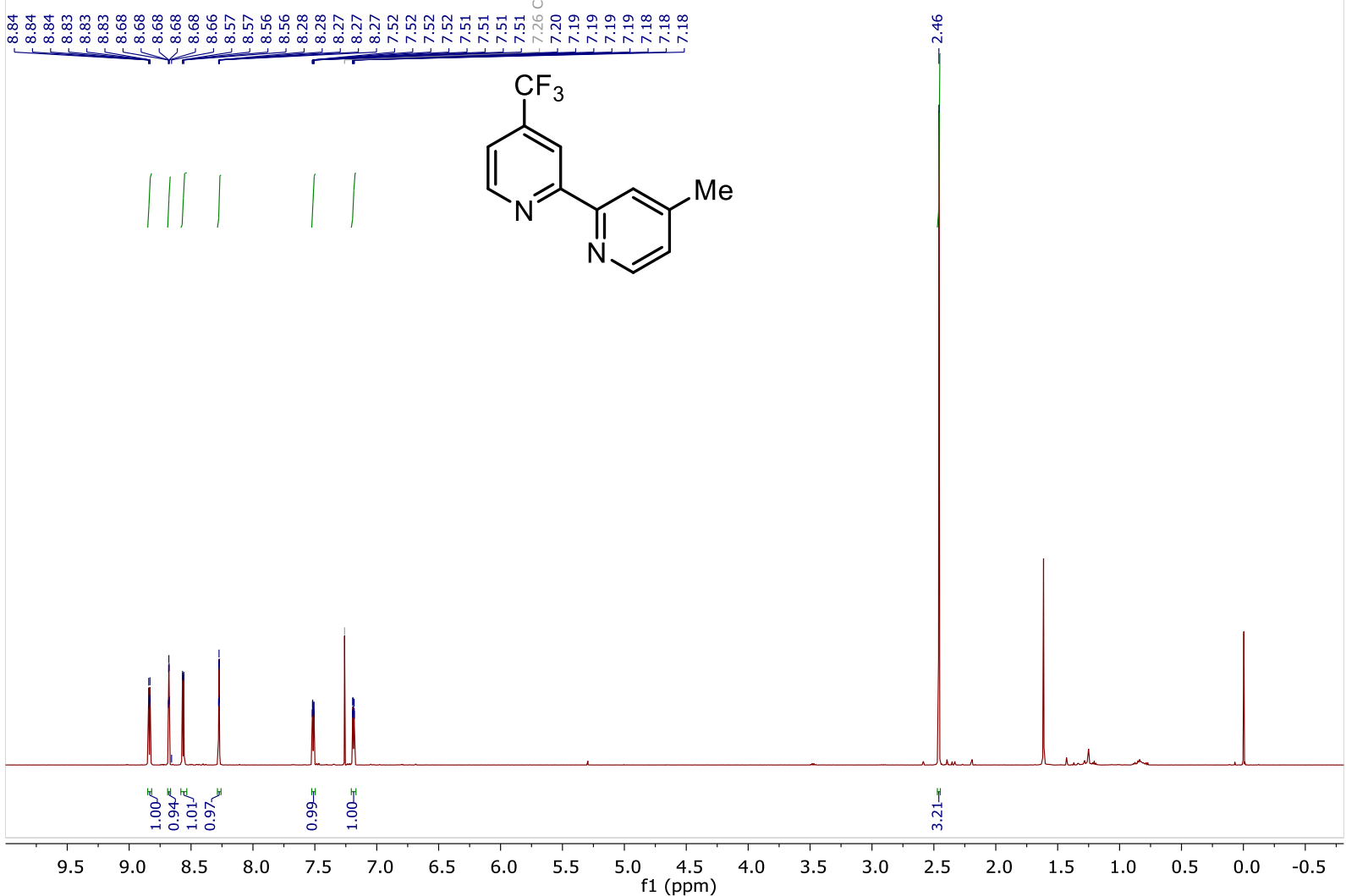

${ }^{13} \mathrm{C}$ NMR (126 MHz, $\left.\mathrm{CDCl}_{3}\right): 5$

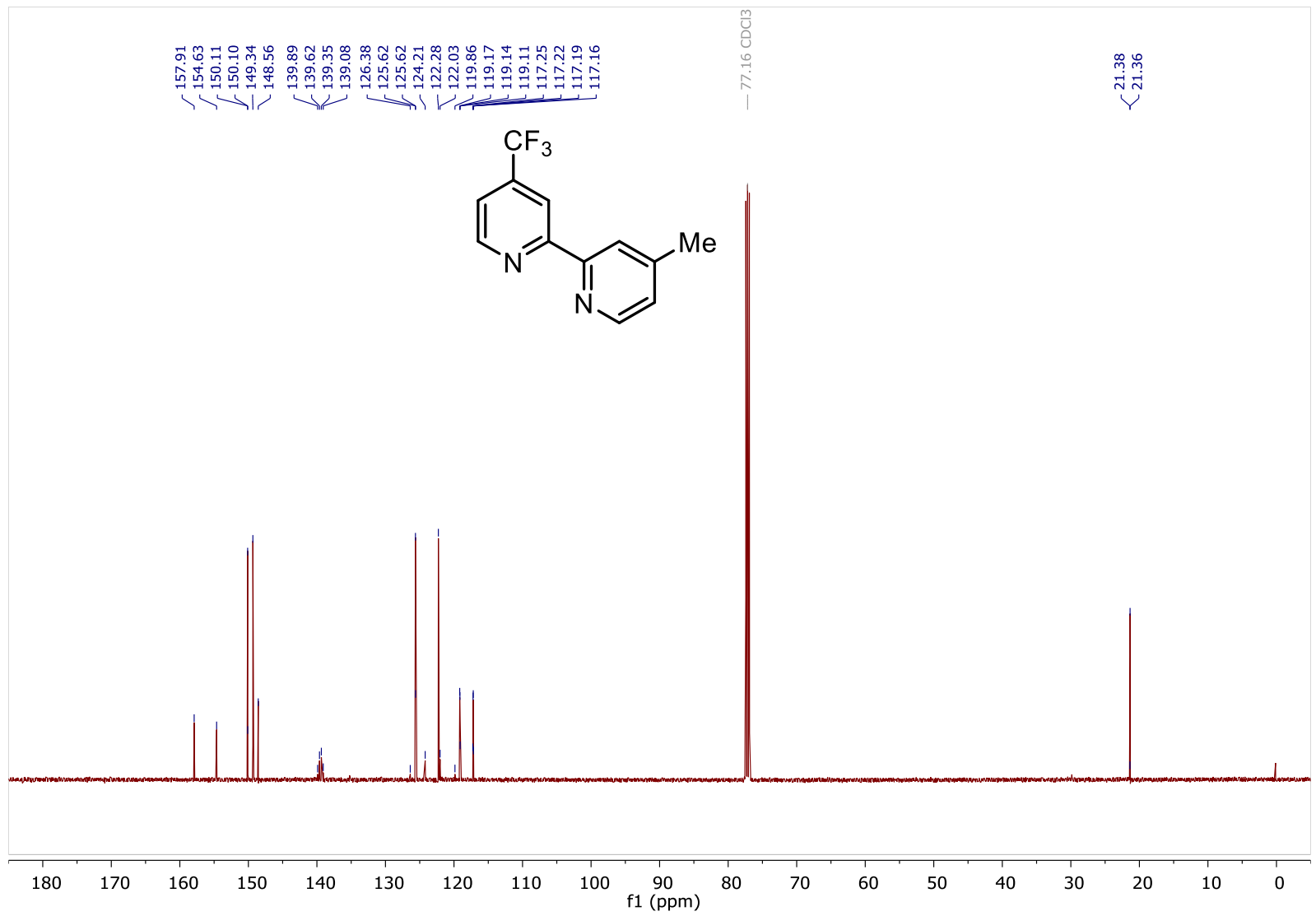


${ }^{1} \mathrm{H}$ NMR (500 MHz, $\left.\mathrm{CDCl}_{3}\right): 6$

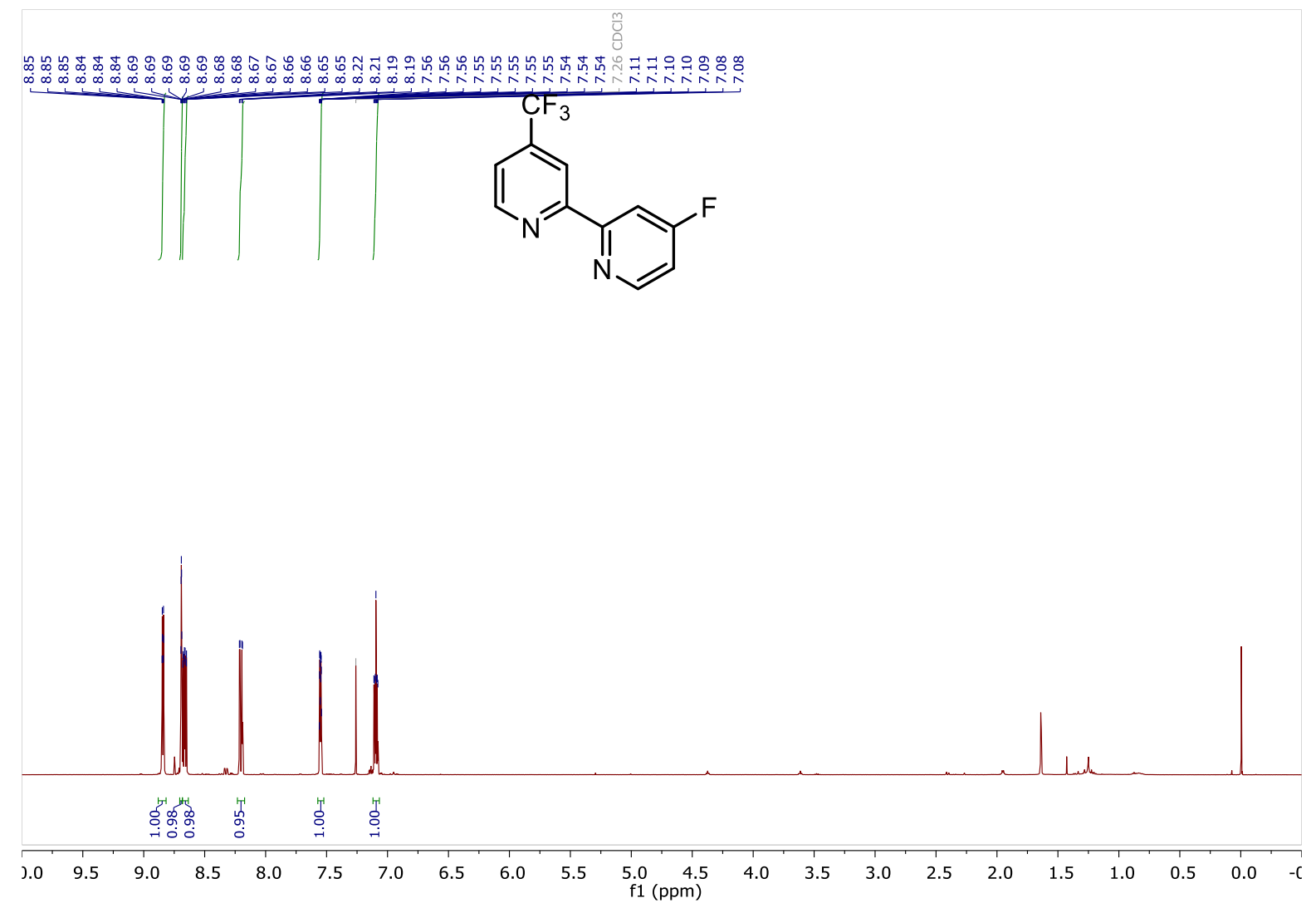

${ }^{13} \mathrm{C}$ NMR (126 MHz, $\left.\mathrm{CDCl}_{3}\right): 6$

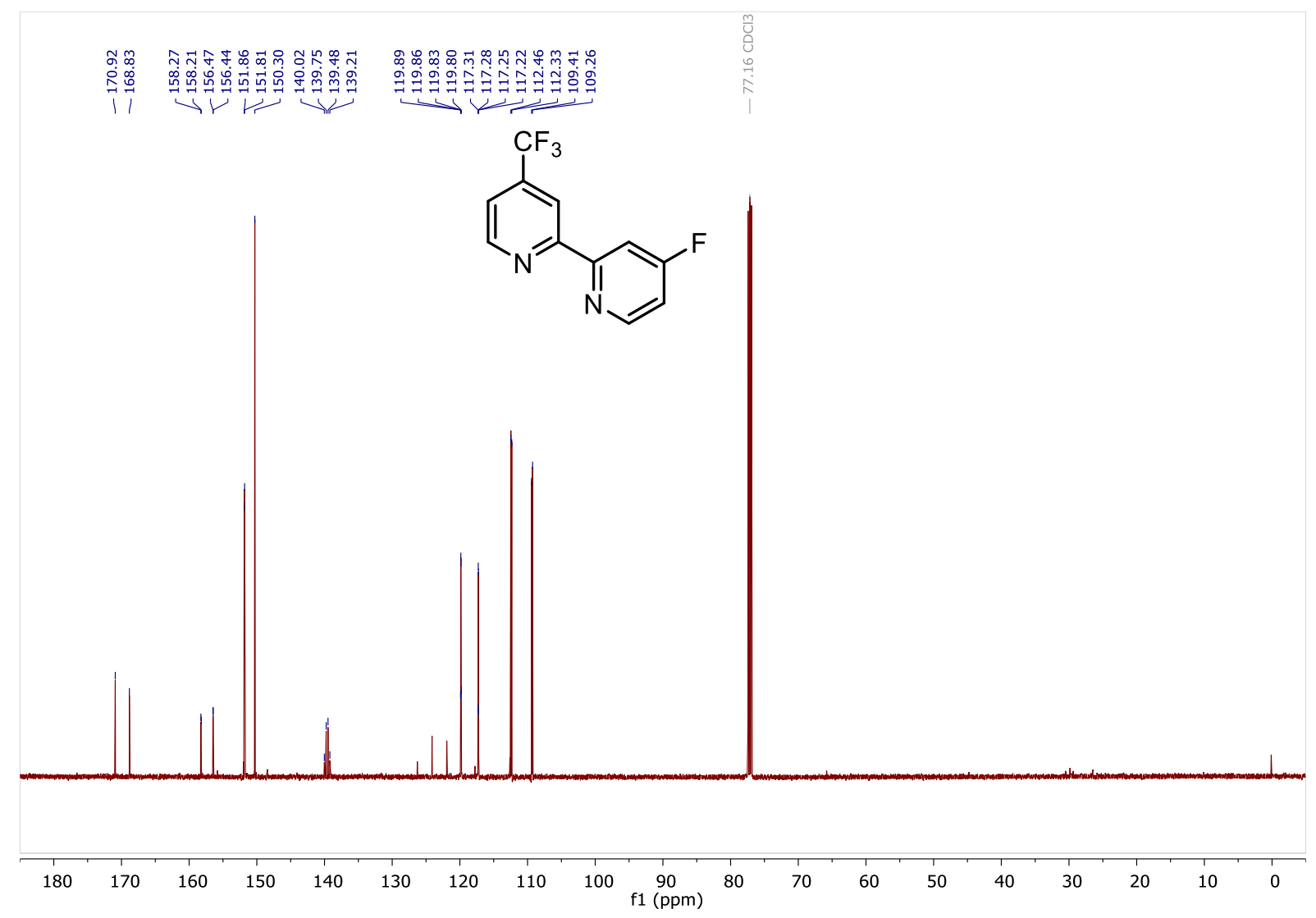


${ }^{1} \mathrm{H}$ NMR (500 MHz, $\left.\mathrm{CDCl}_{3}\right): 7$

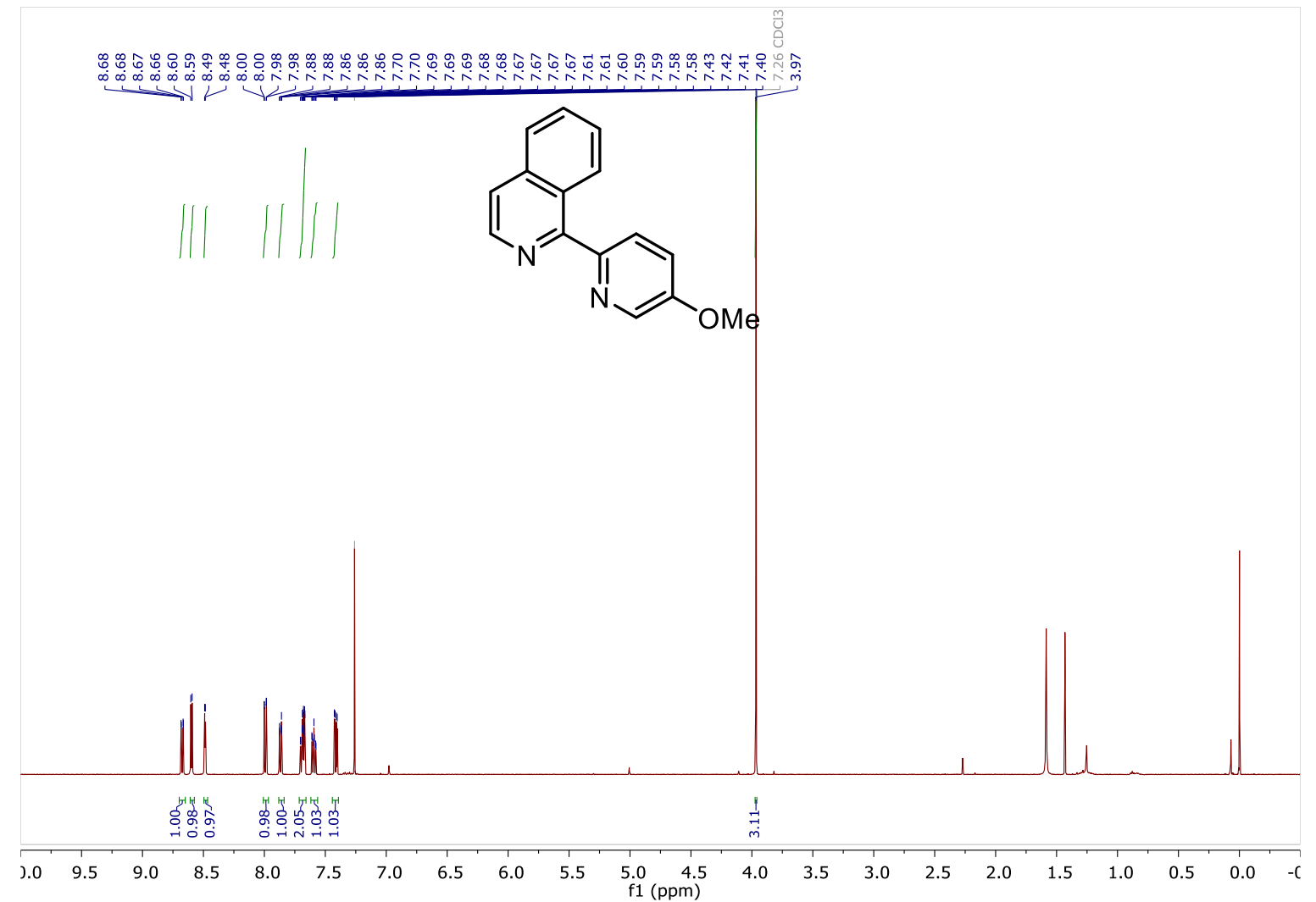

${ }^{13} \mathrm{C}$ NMR (126 MHz, $\left.\mathrm{CDCl}_{3}\right): 7$

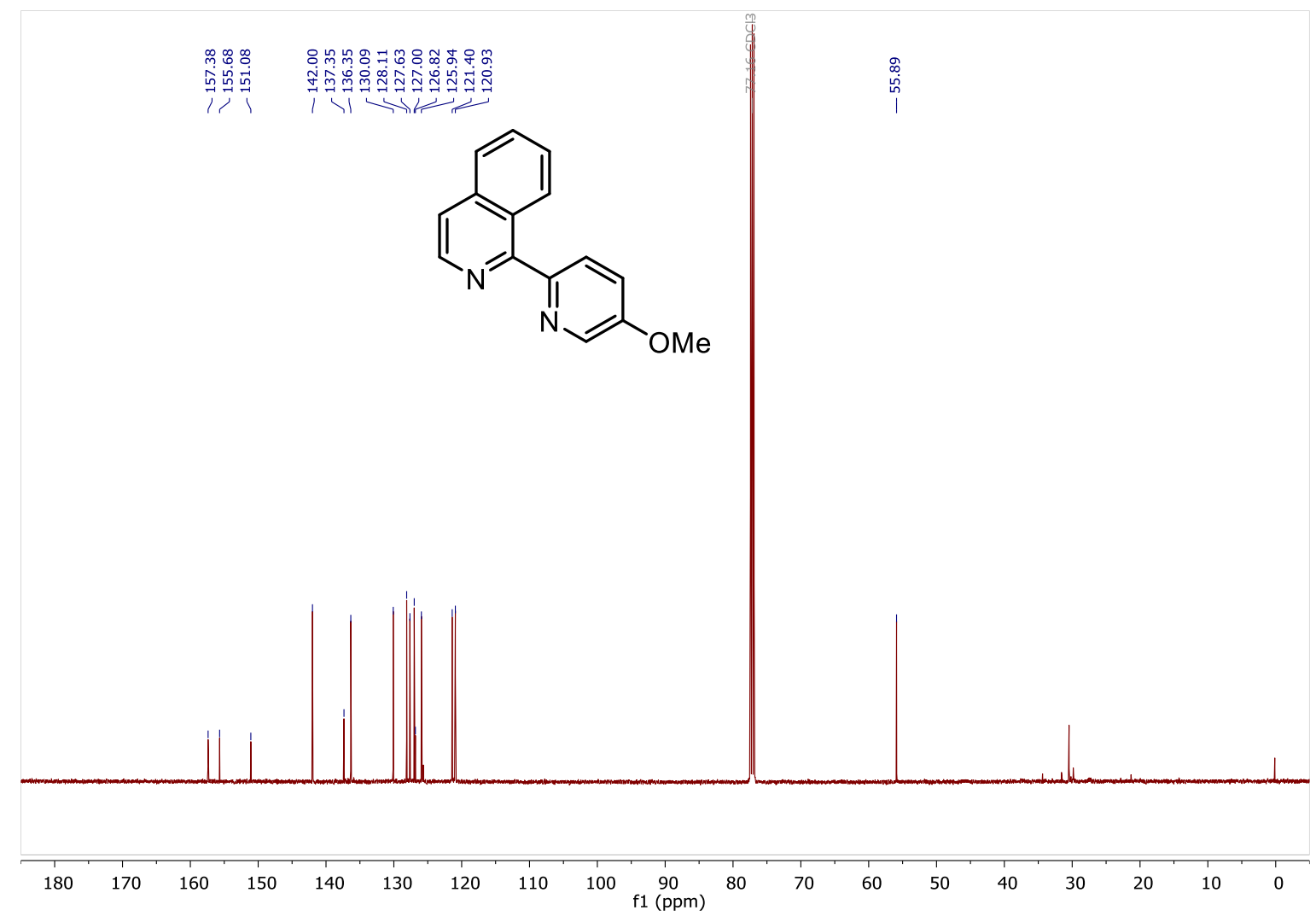


${ }^{1} \mathrm{H}$ NMR (500 MHz, $\left.\mathrm{CDCl}_{3}\right): 8$

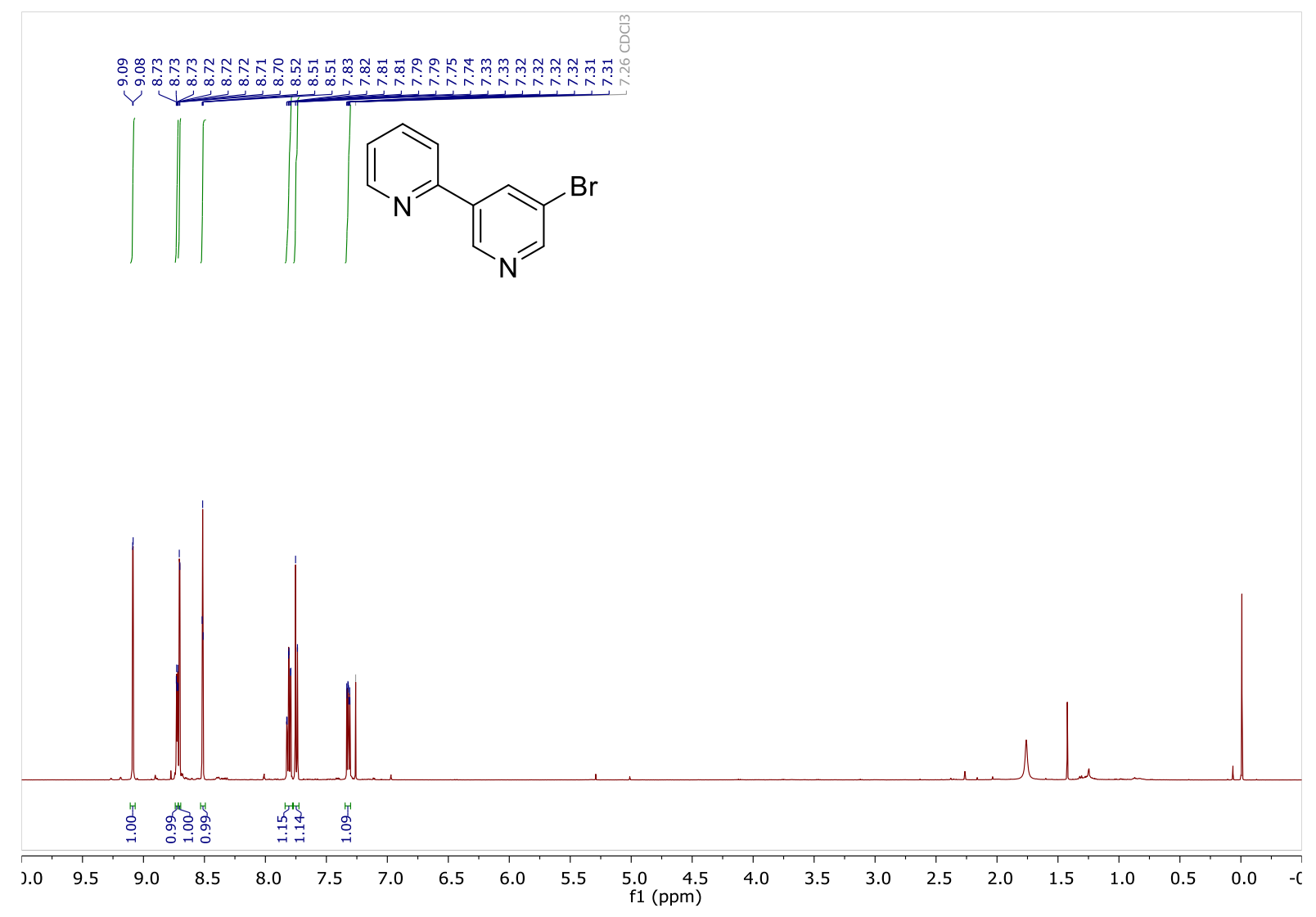

${ }^{13} \mathrm{C}$ NMR (126 MHz, $\left.\mathrm{CDCl}_{3}\right): 8$

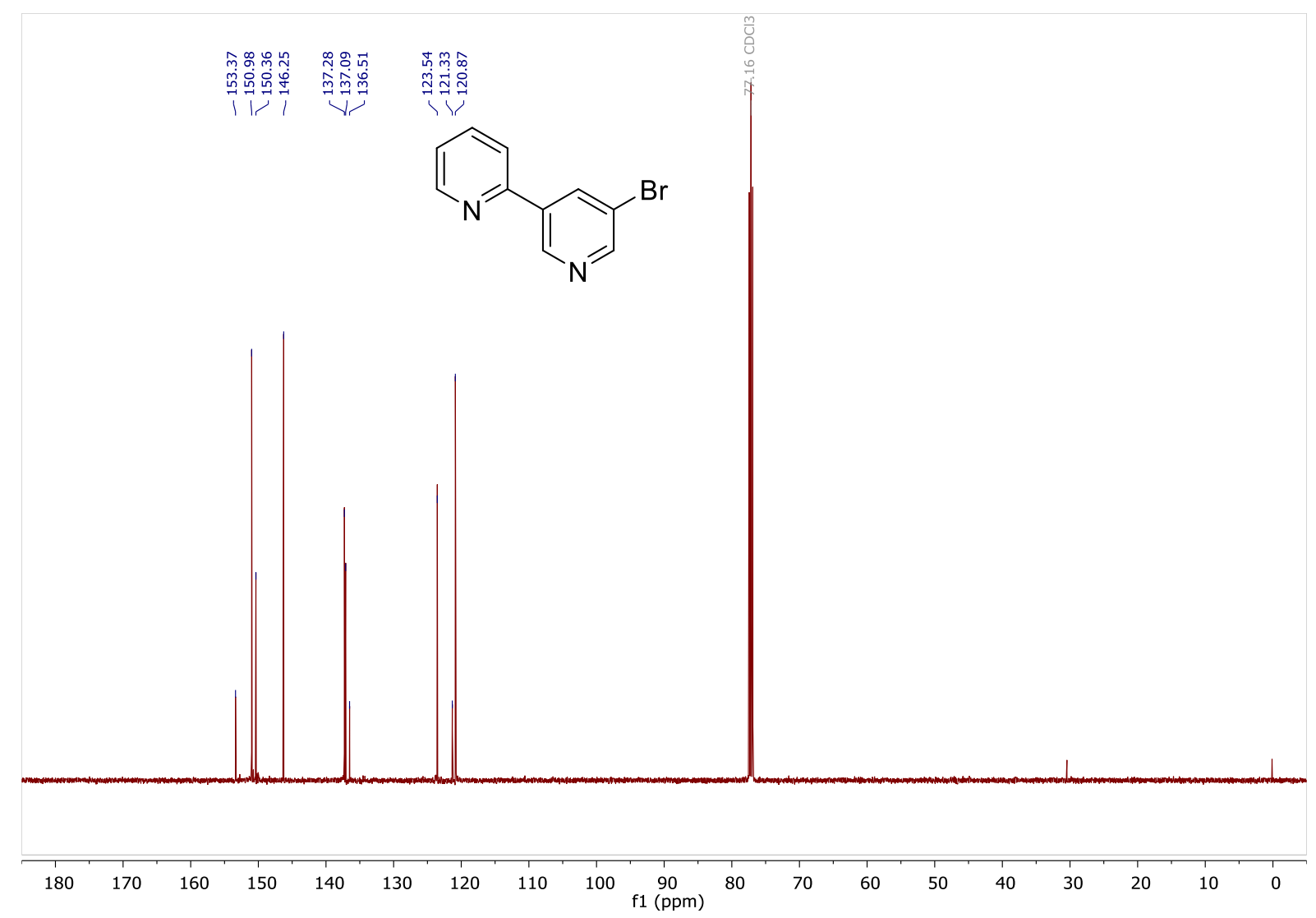


${ }^{1} \mathrm{H}$ NMR (500 MHz, $\left.\mathrm{CDCl}_{3}\right): 9$

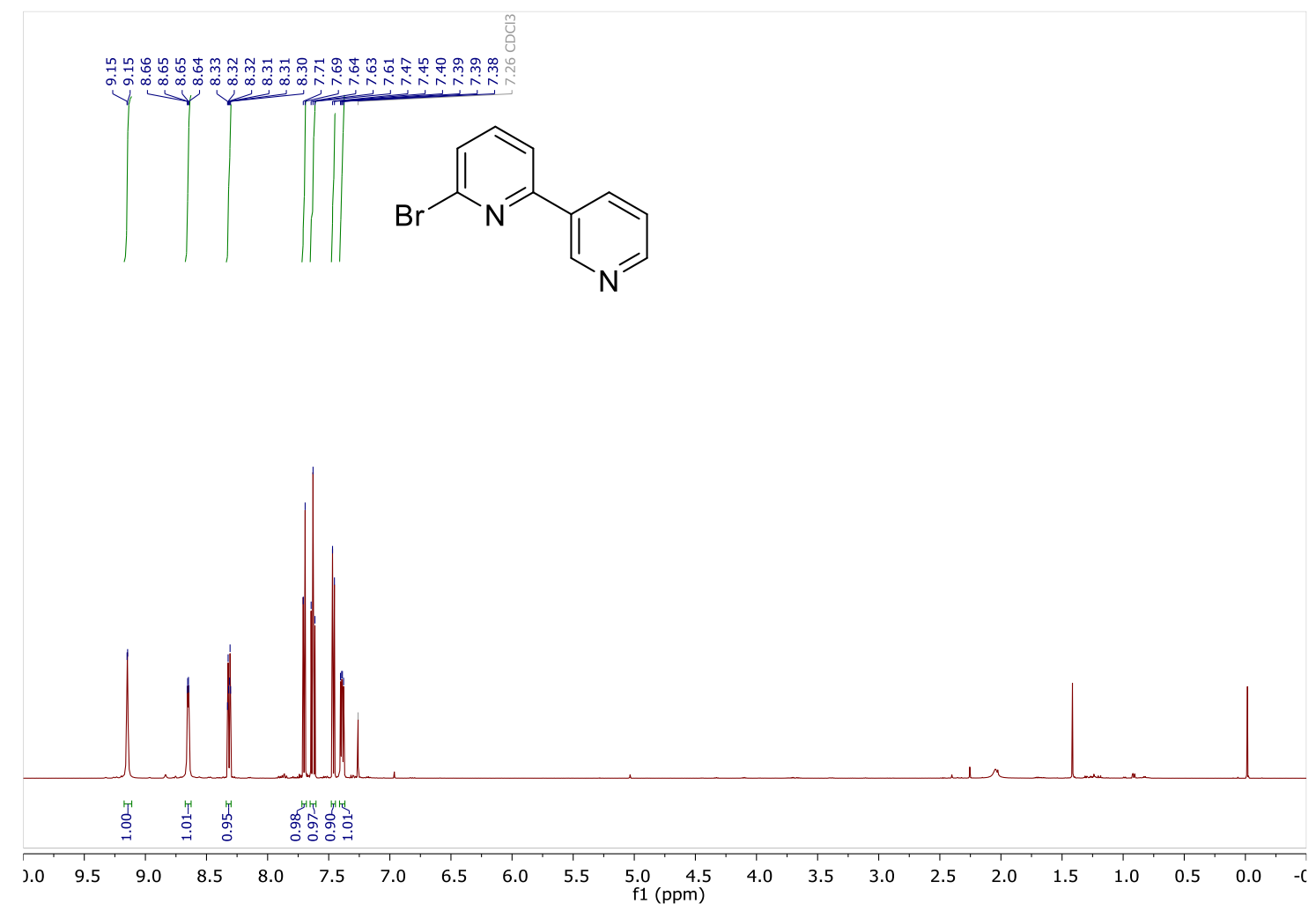

${ }^{13} \mathrm{C}$ NMR (126 MHz, $\left.\mathrm{CDCl}_{3}\right): 9$

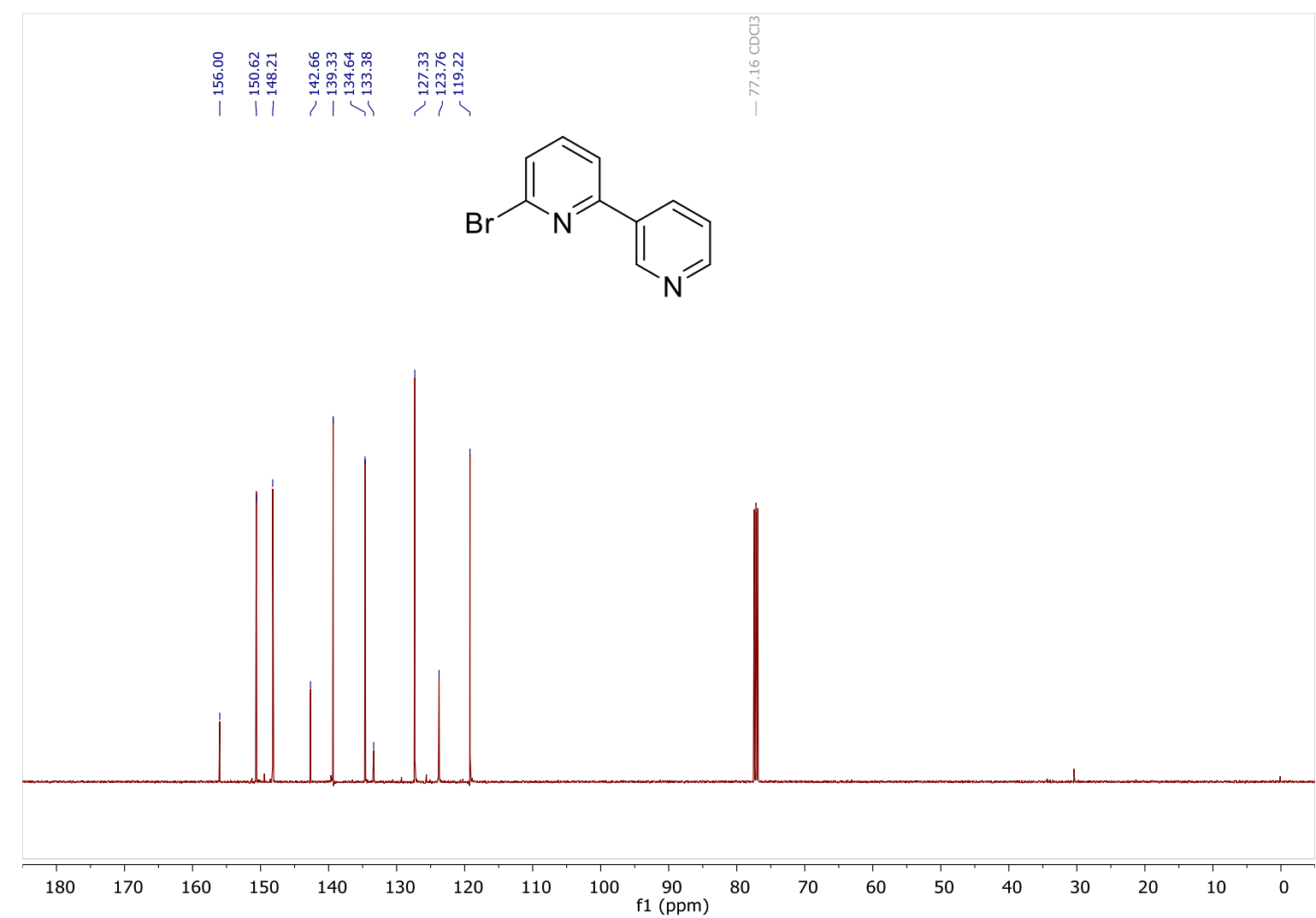


${ }^{1} \mathrm{H}$ NMR (400 MHz, $\mathrm{CDCl}_{3}$ ): 10

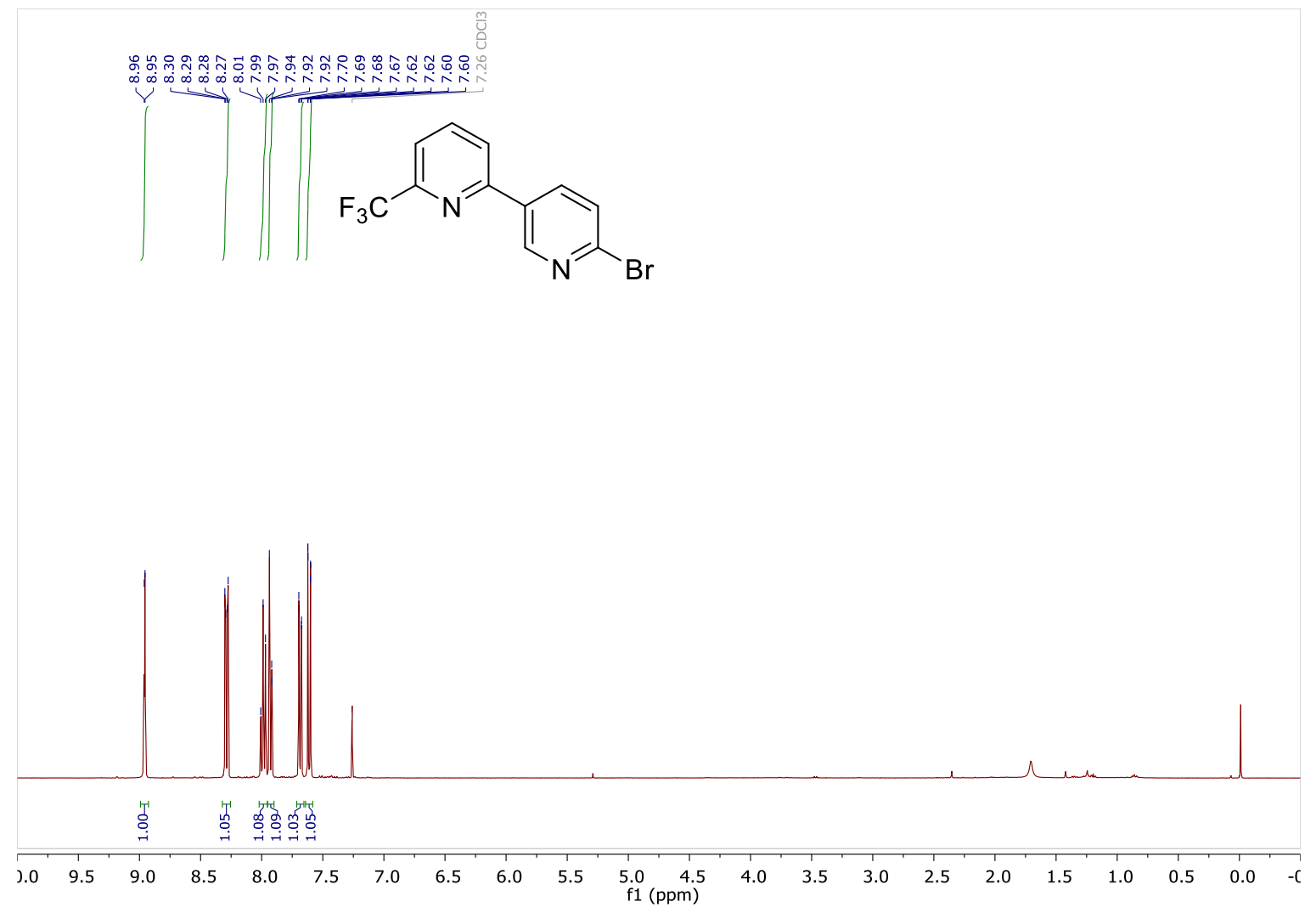

${ }^{13} \mathrm{C}$ NMR (101 MHz, $\left.\mathrm{CDCl}_{3}\right): 10$

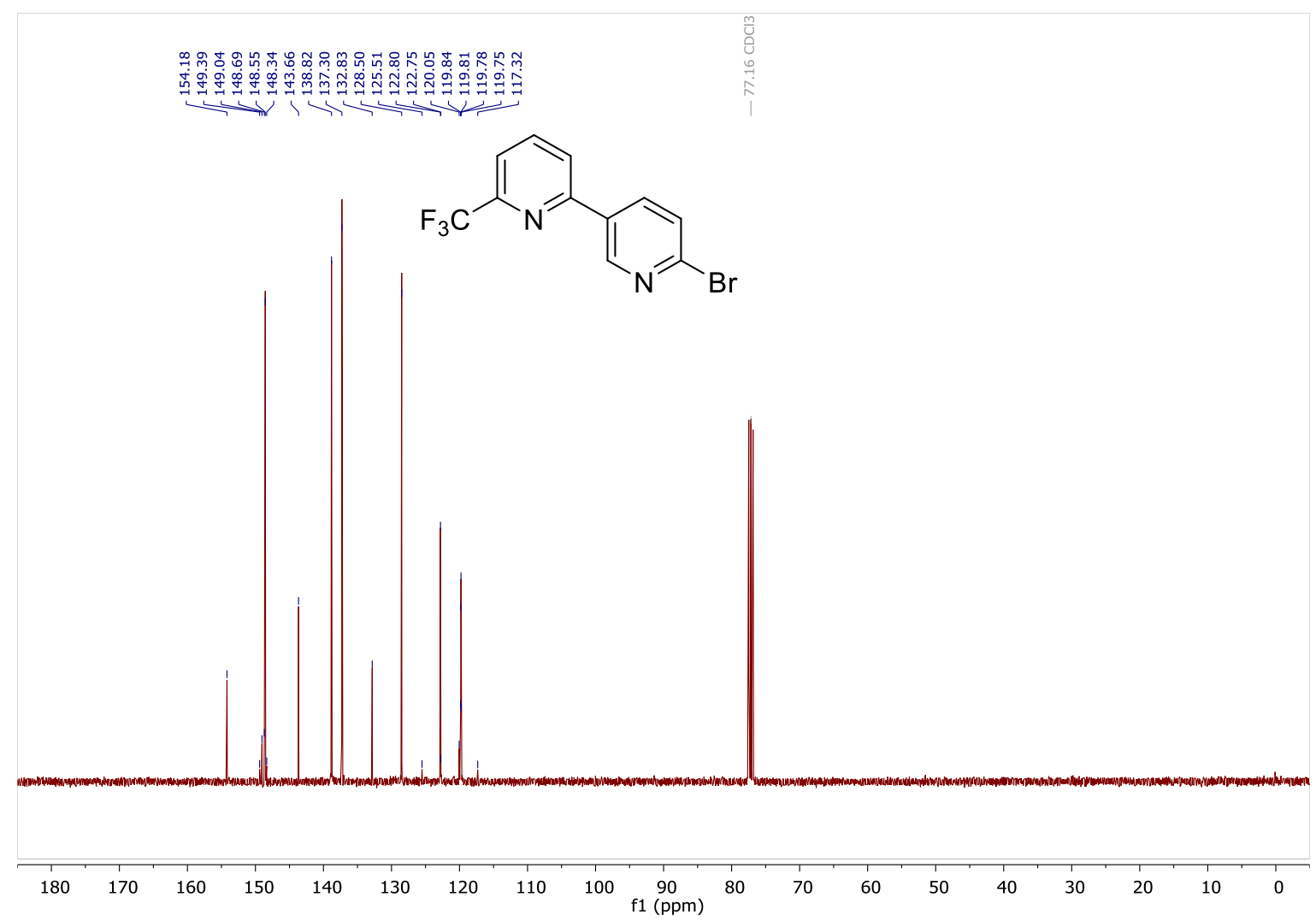


${ }^{1} \mathrm{H}$ NMR (400 MHz, $\mathrm{CDCl}_{3}$ ): 11

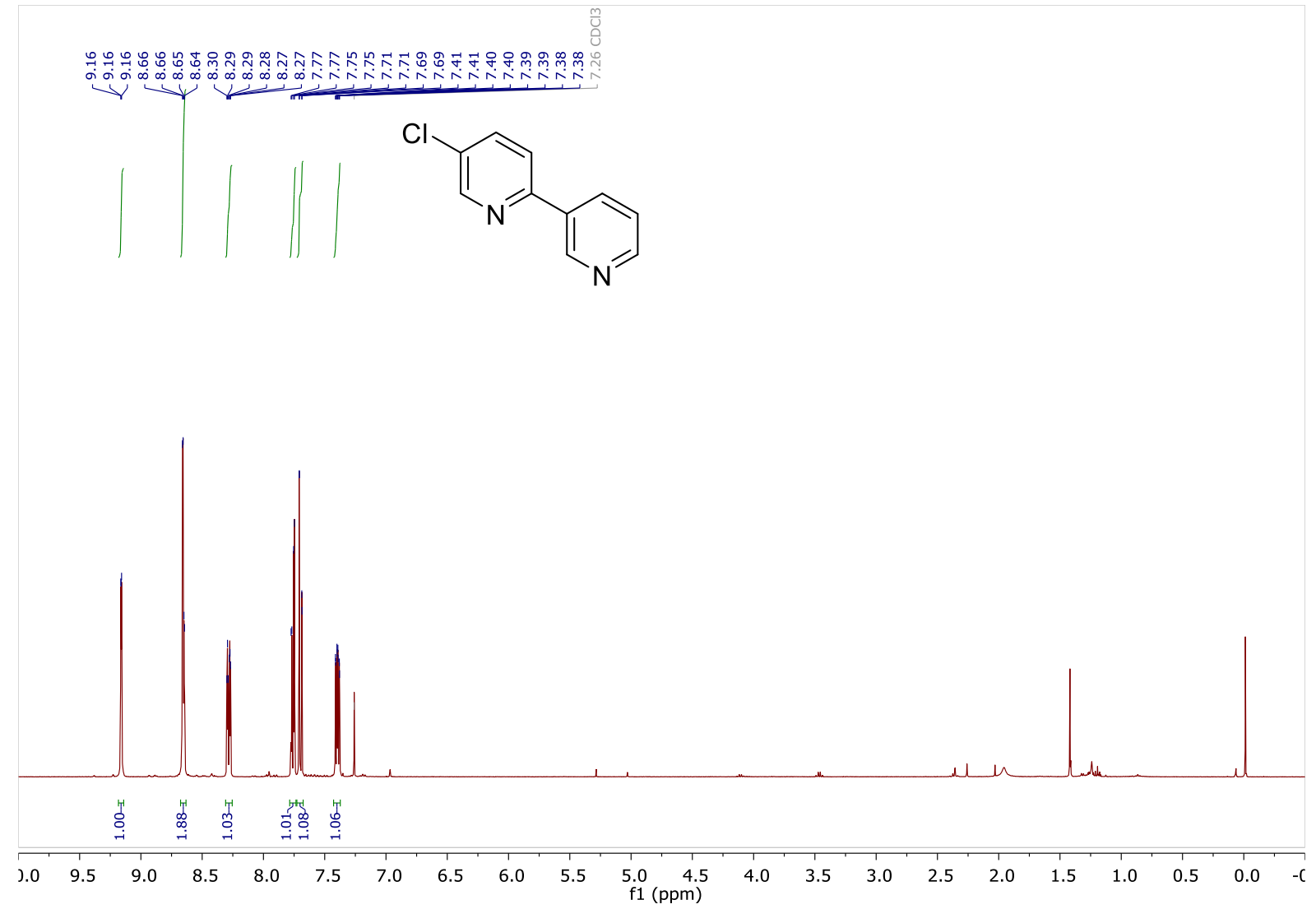

${ }^{13}$ C NMR (101 MHz, $\left.\mathrm{CDCl}_{3}\right): 11$

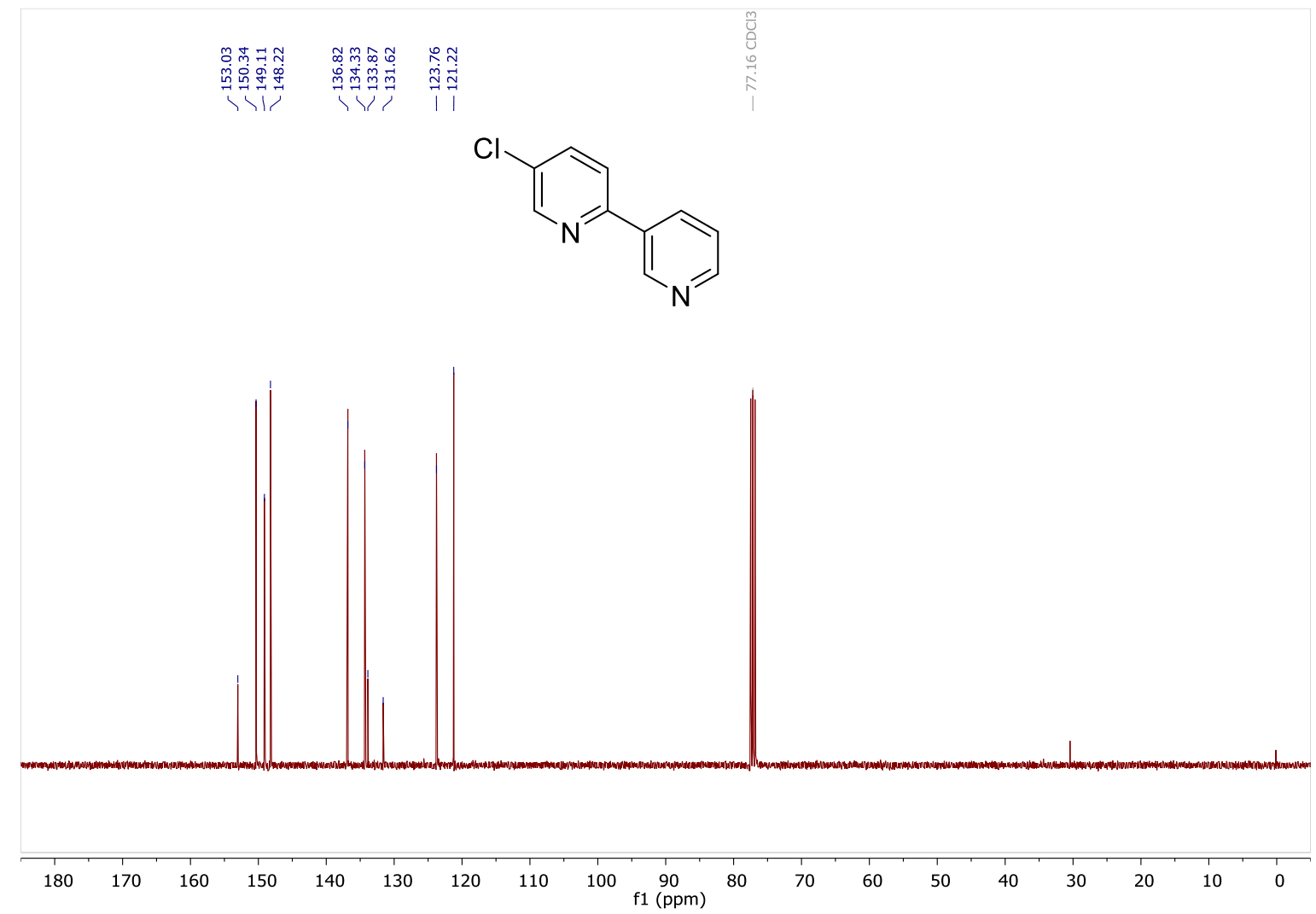


${ }^{1} \mathrm{H}$ NMR (400 MHz, $\mathrm{CDCl}_{3}$ ): 12

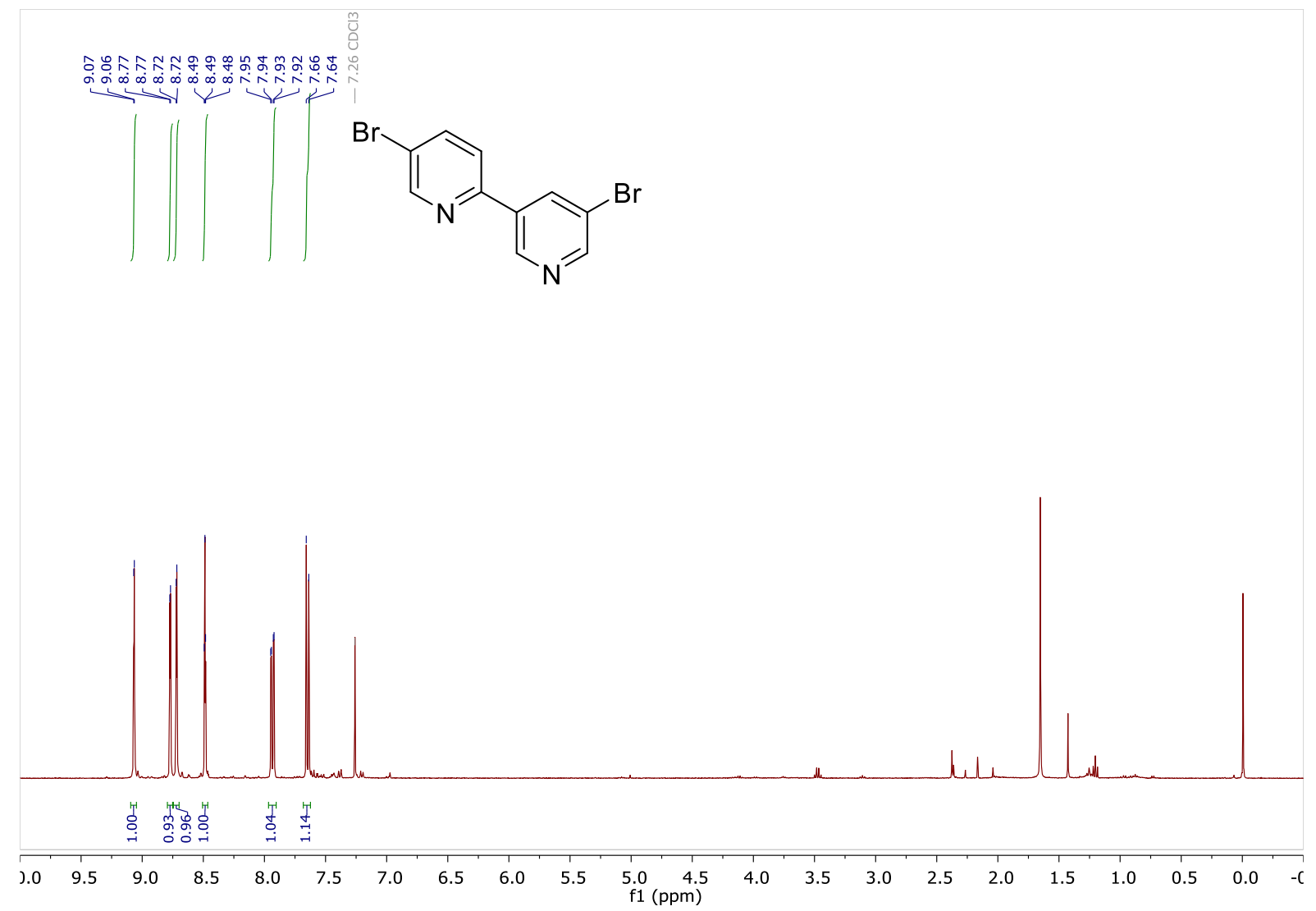

${ }^{13} \mathrm{C}$ NMR (101 MHz, $\left.\mathrm{CDCl}_{3}\right): 12$

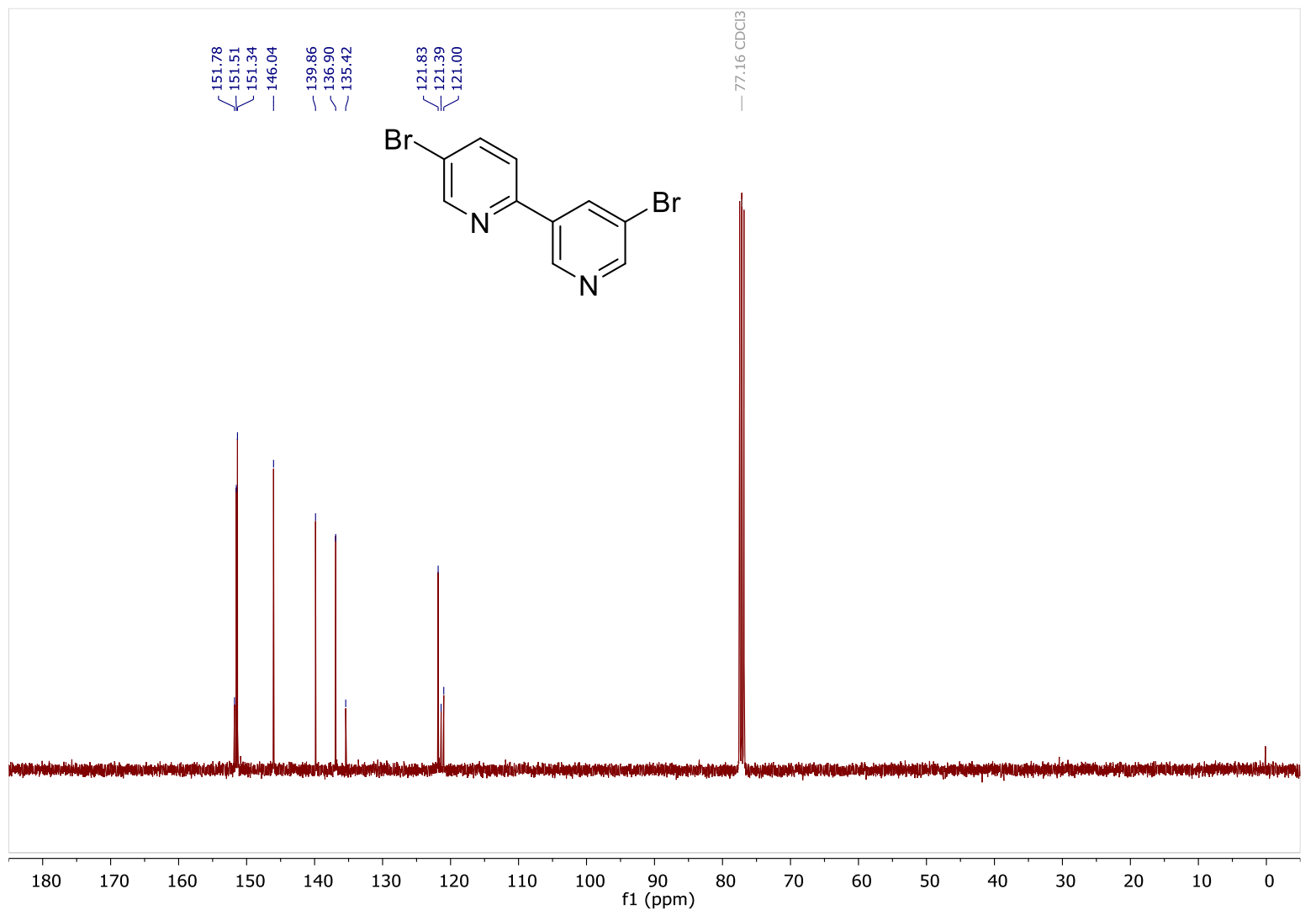


${ }^{1} \mathrm{H}$ NMR (500 MHz, $\left.\mathrm{CDCl}_{3}\right): 13$

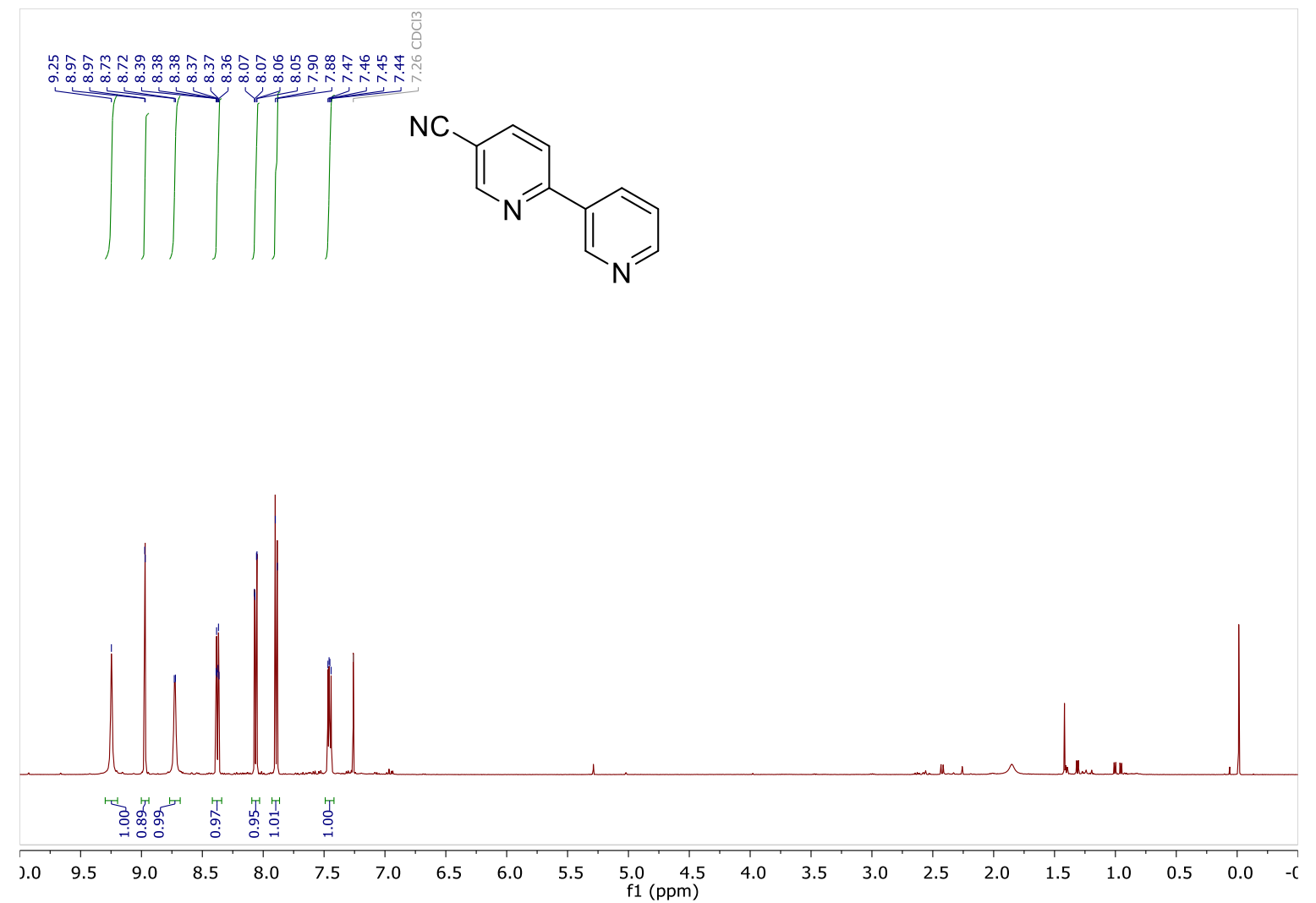

${ }^{13} \mathrm{C}$ NMR (126 MHz, $\left.\mathrm{CDCl}_{3}\right): 13$

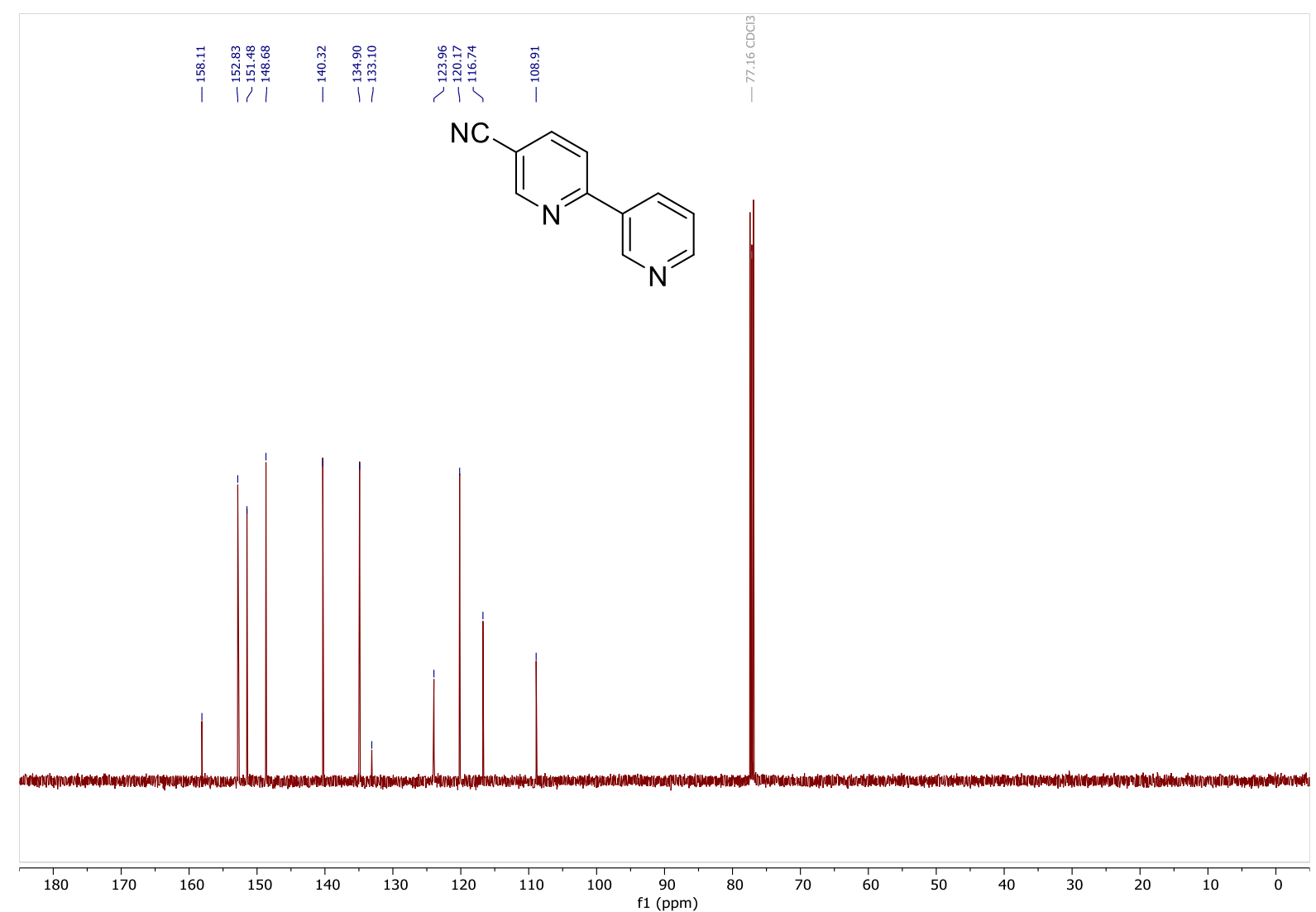


${ }^{1} \mathrm{H}$ NMR (500 MHz, $\mathrm{CDCl}_{3}$ ): 14

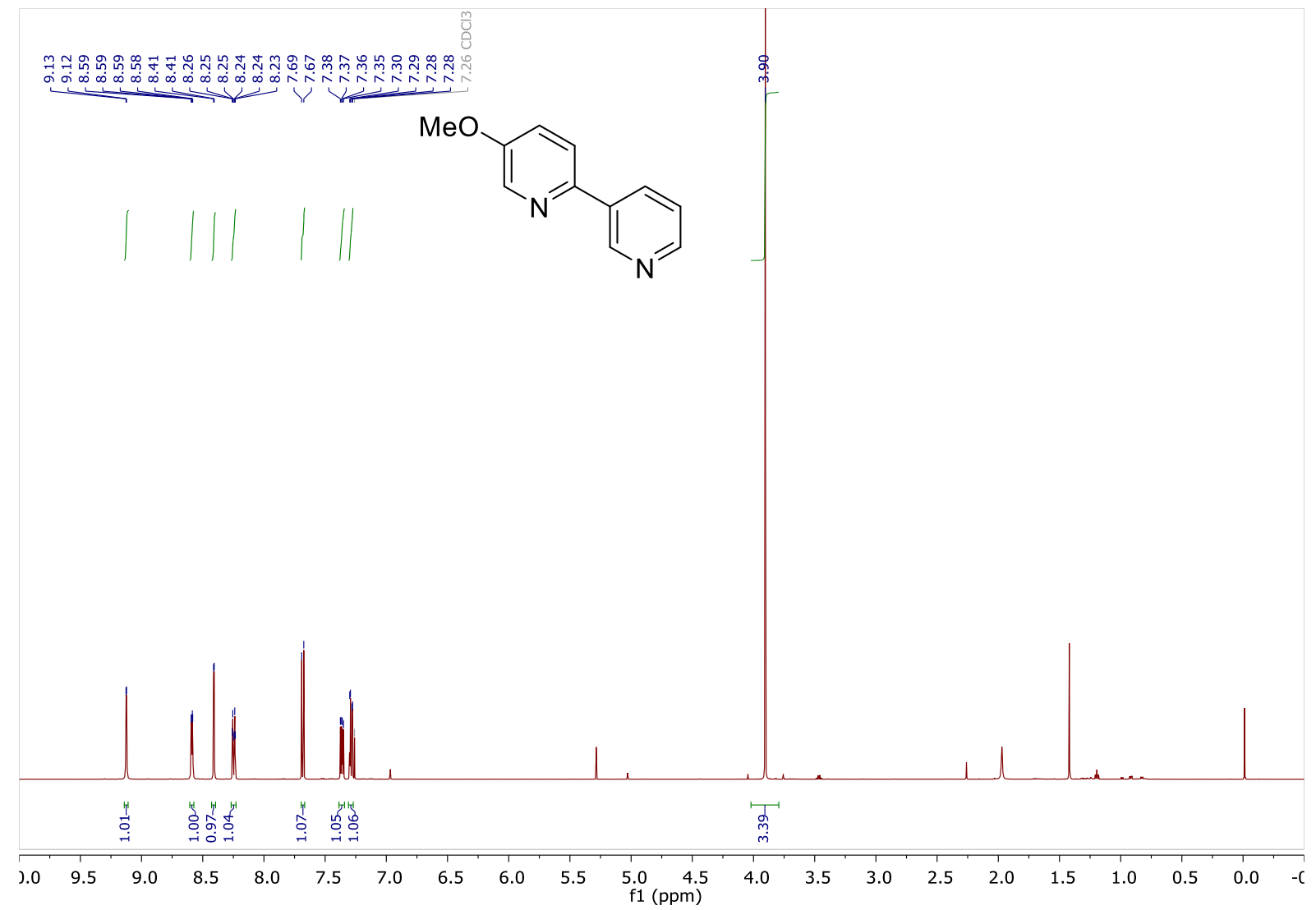

${ }^{13}$ C NMR (126 MHz, $\left.\mathrm{CDCl}_{3}\right): 14$

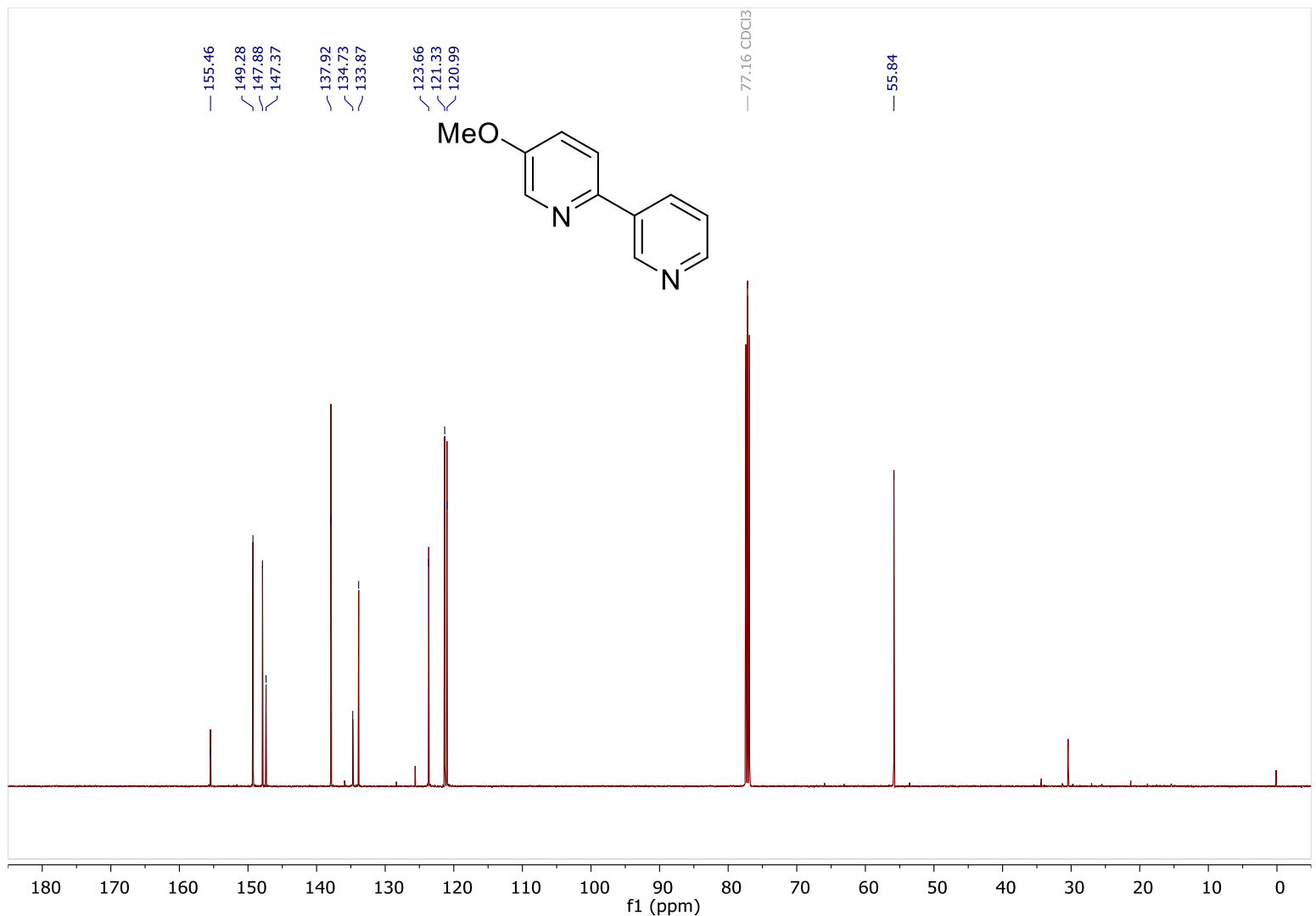


${ }^{1} \mathrm{H}$ NMR (500 MHz, $\left.\mathrm{CDCl}_{3}\right): 15$

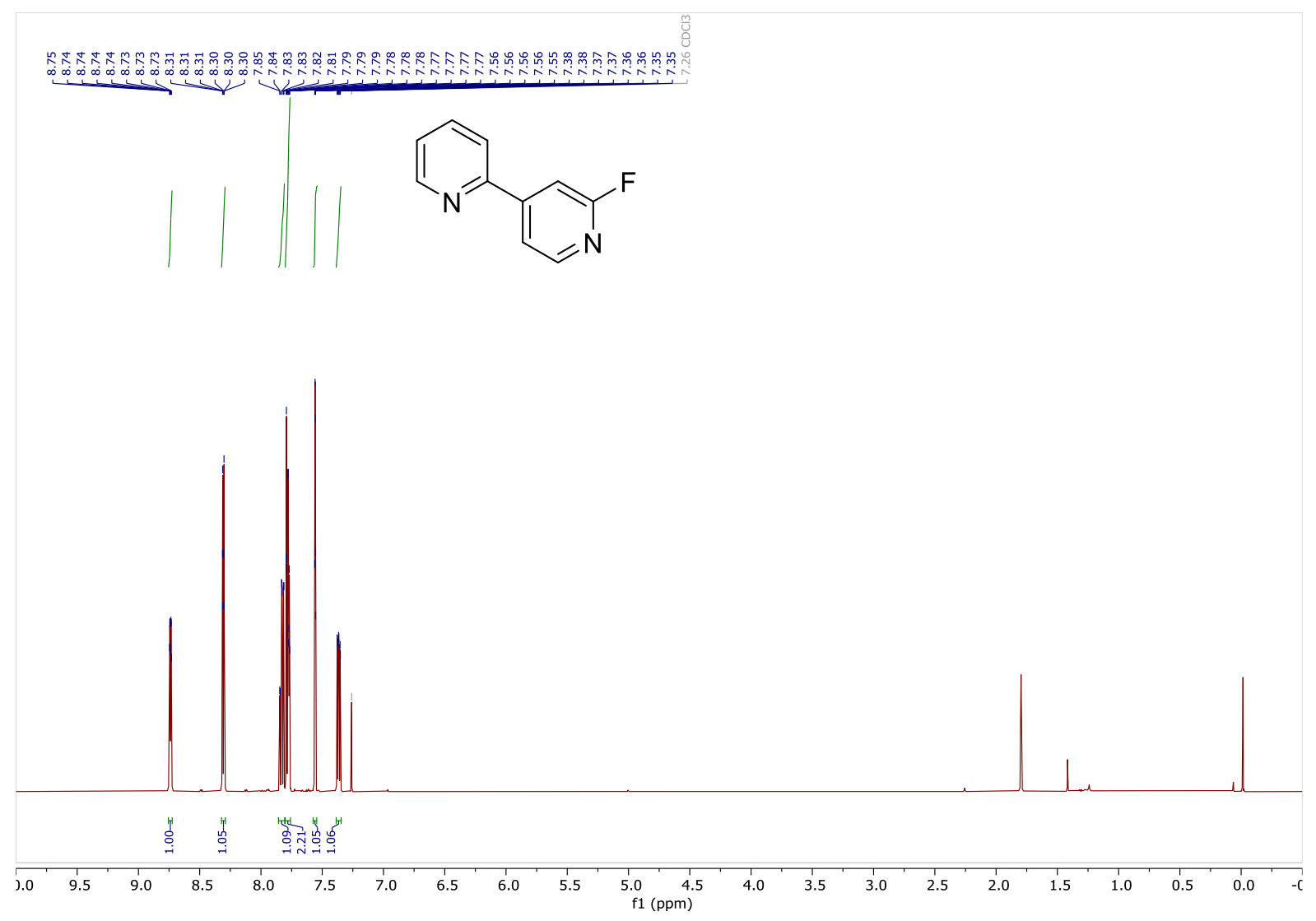

${ }^{13} \mathrm{C}$ NMR (126 MHz, $\left.\mathrm{CDCl}_{3}\right): 15$

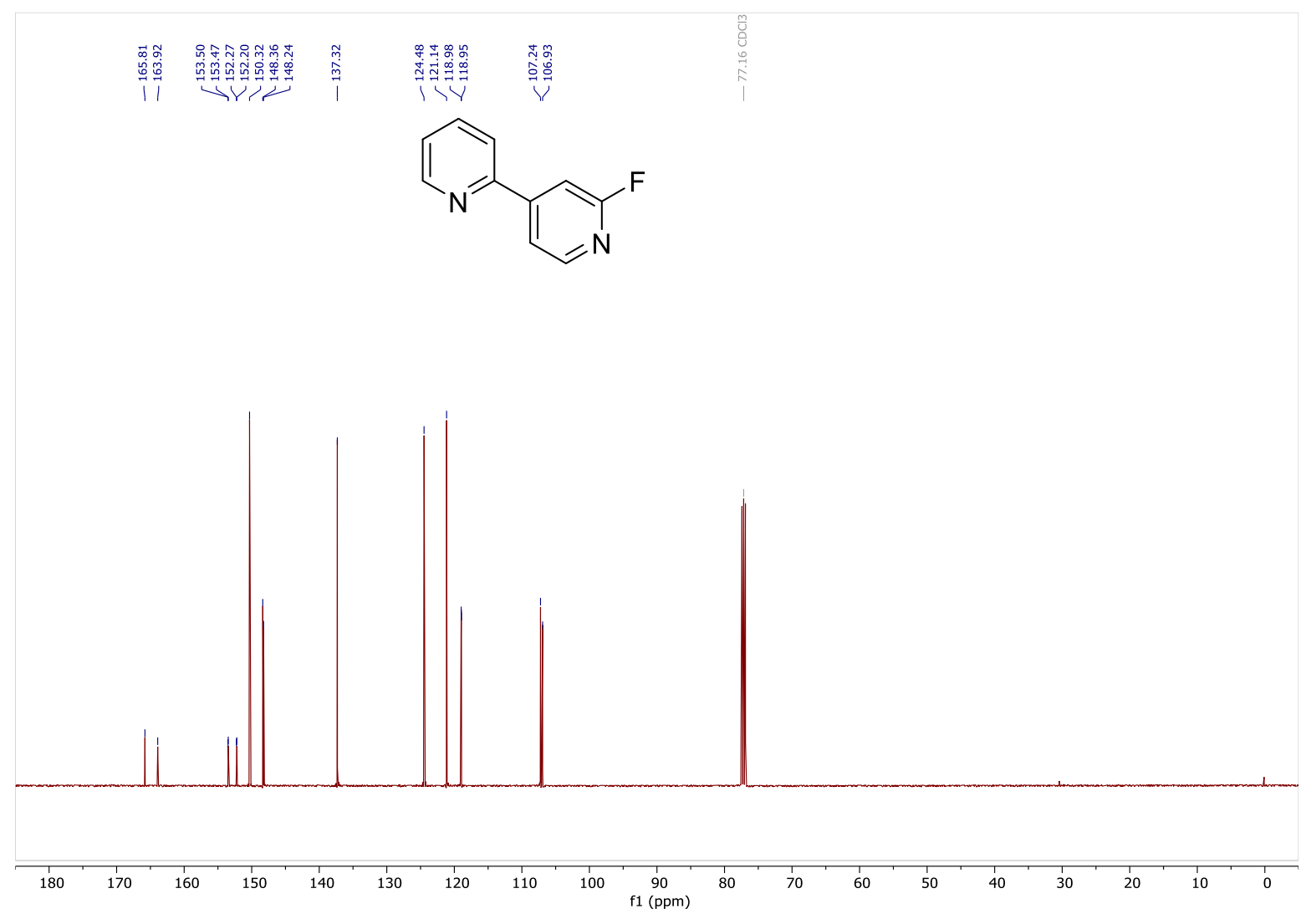

55 
${ }^{1} \mathrm{H}$ NMR (500 MHz, $\mathrm{CDCl}_{3}$ ): 16

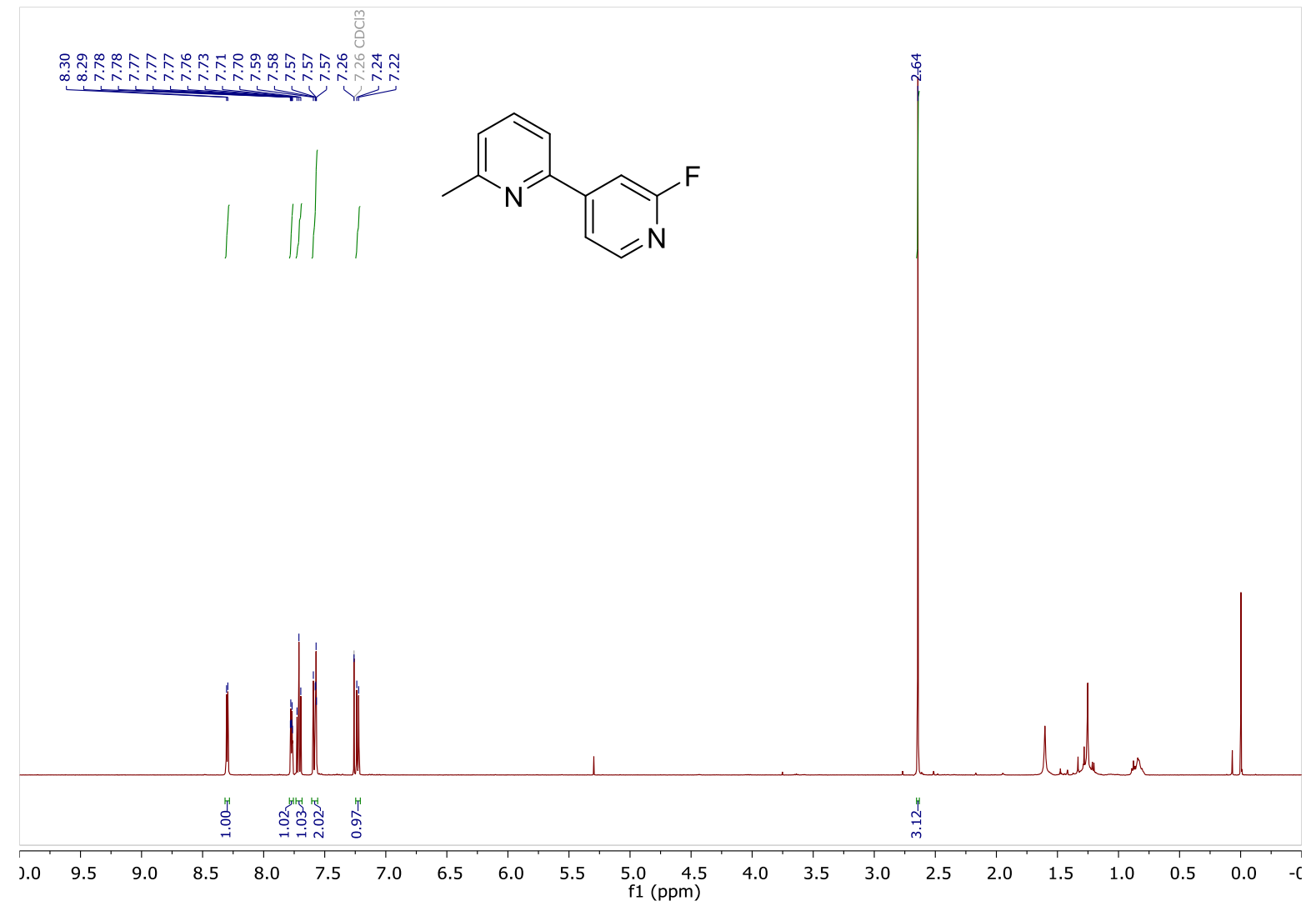

${ }^{13} \mathrm{C}$ NMR (126 MHz, $\left.\mathrm{CDCl}_{3}\right): 16$

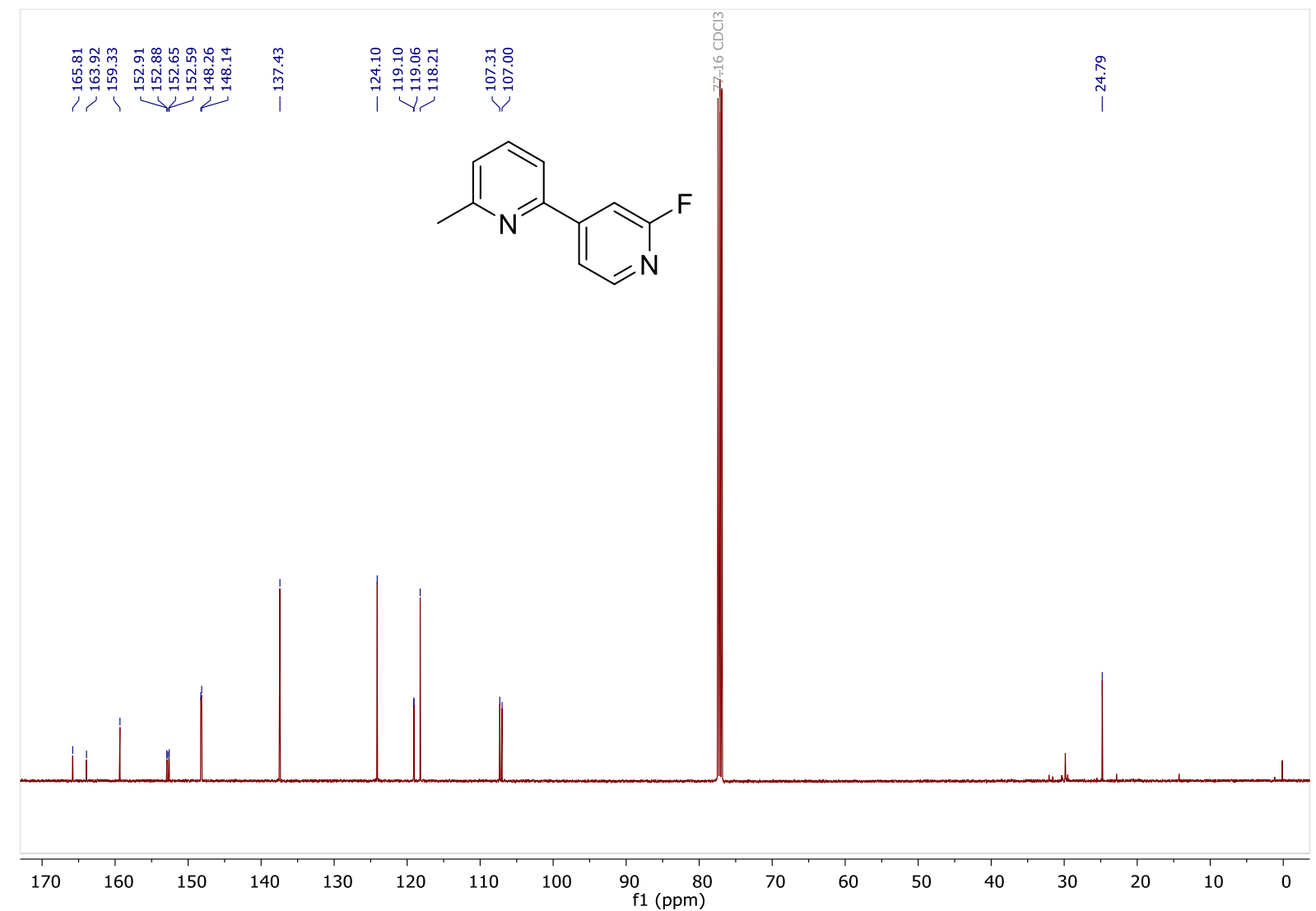


${ }^{1} \mathrm{H}$ NMR (500 MHz, $\mathrm{CDCl}_{3}$ ): 17

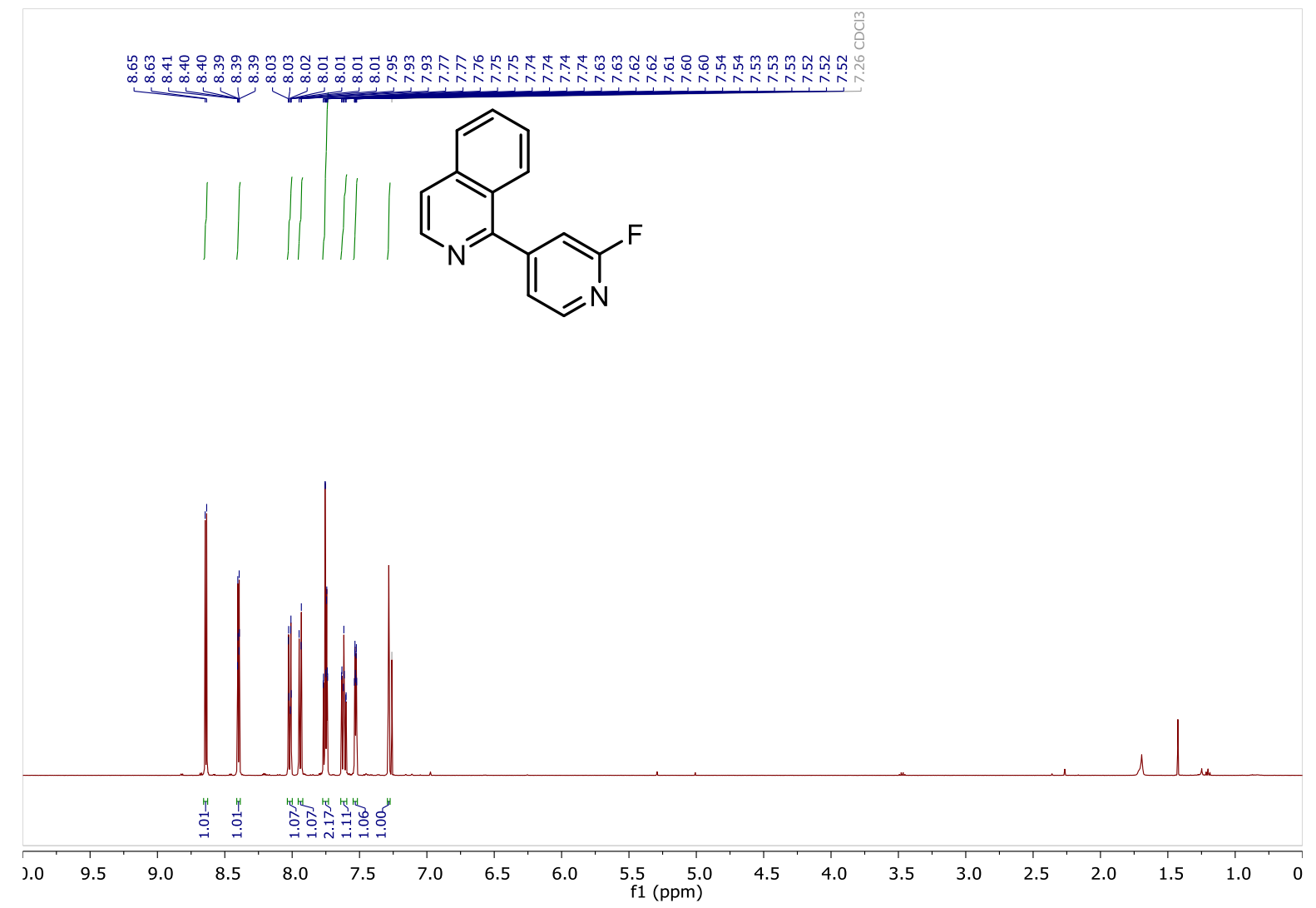

${ }^{13}$ C NMR (126 MHz, $\left.\mathrm{CDCl}_{3}\right): 17$

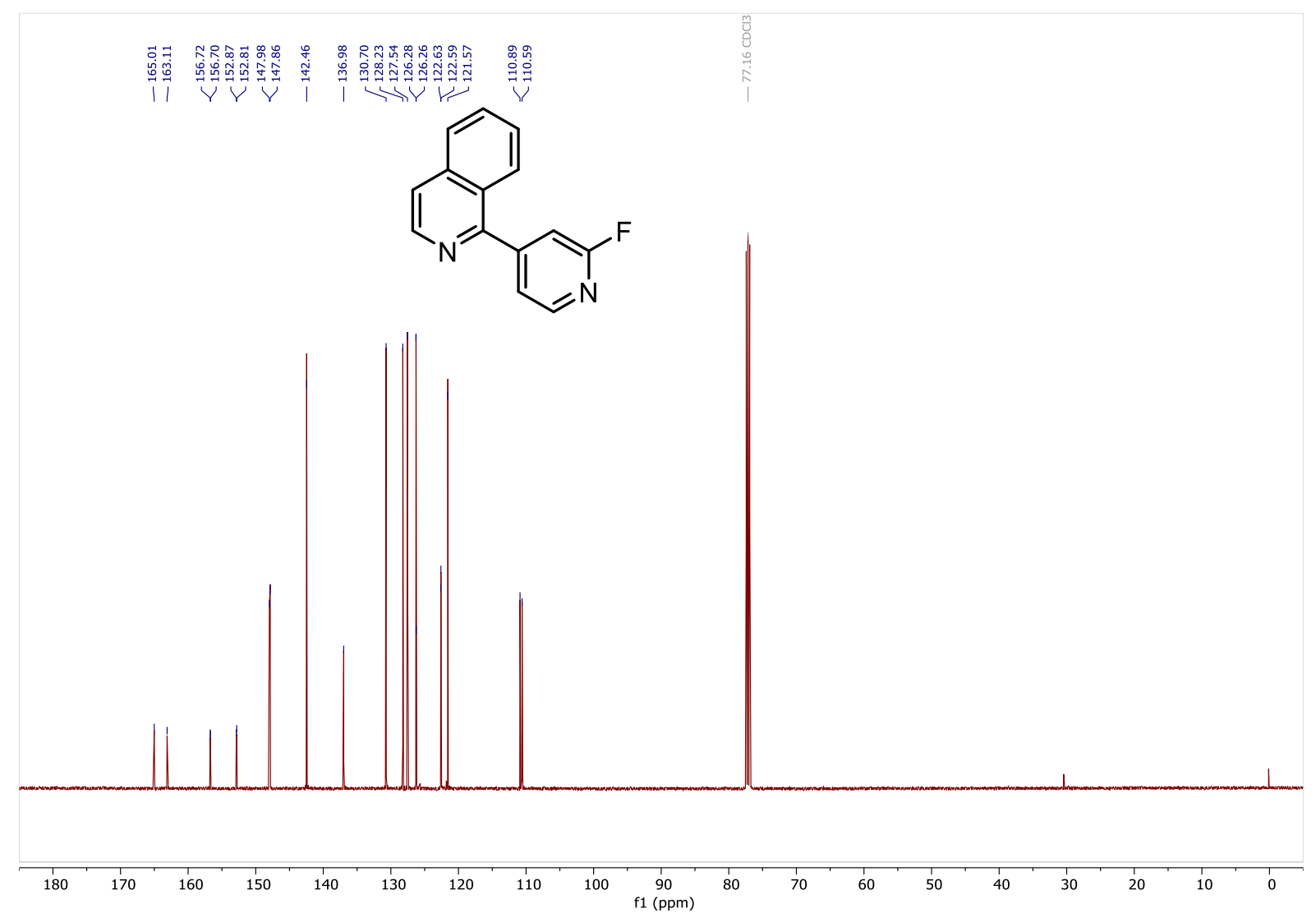


${ }^{1} \mathrm{H}$ NMR (500 MHz, $\mathrm{CDCl}_{3}$ ): 18

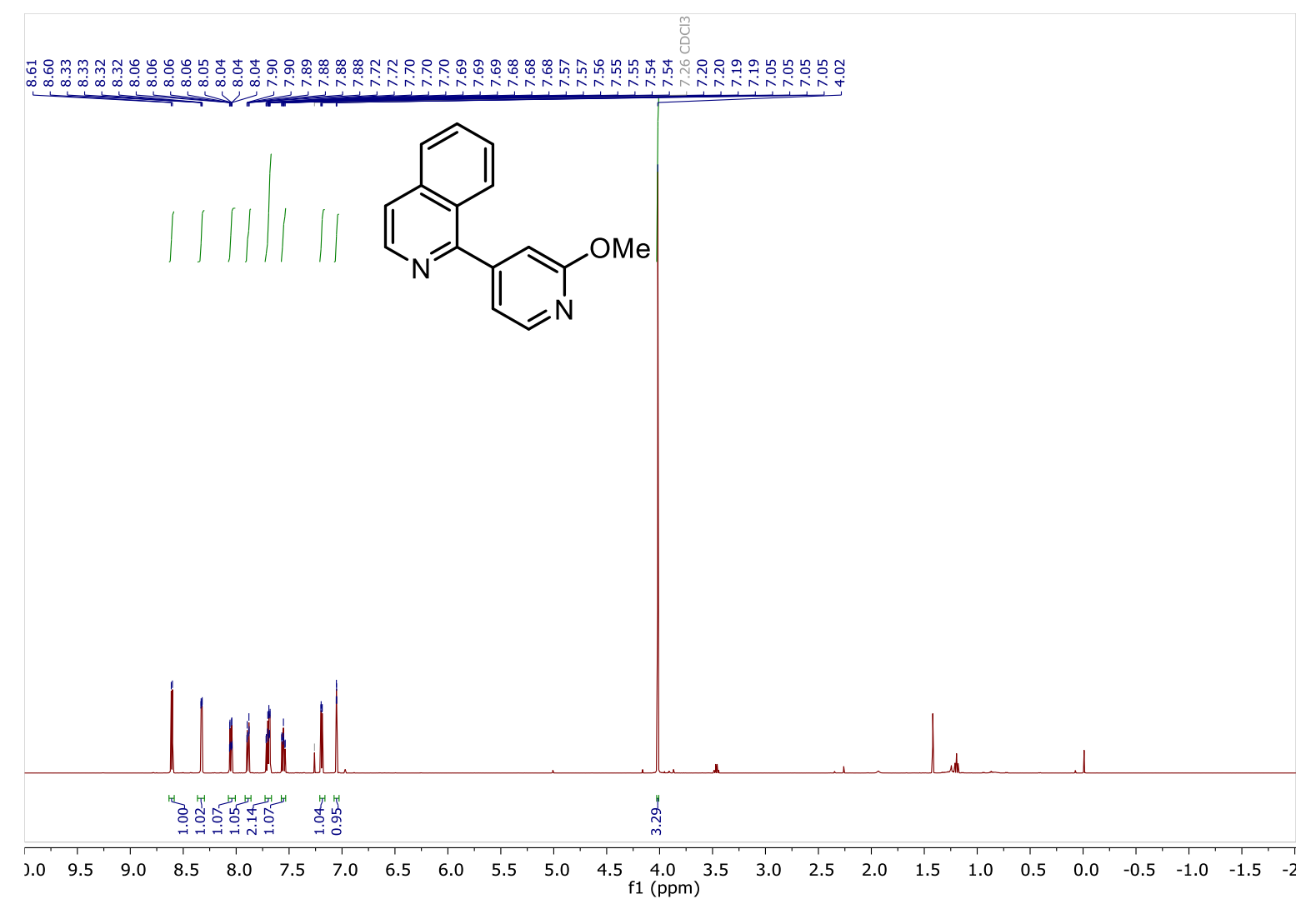

${ }^{13} \mathrm{C}$ NMR (126 MHz, $\left.\mathrm{CDCl}_{3}\right): 18$

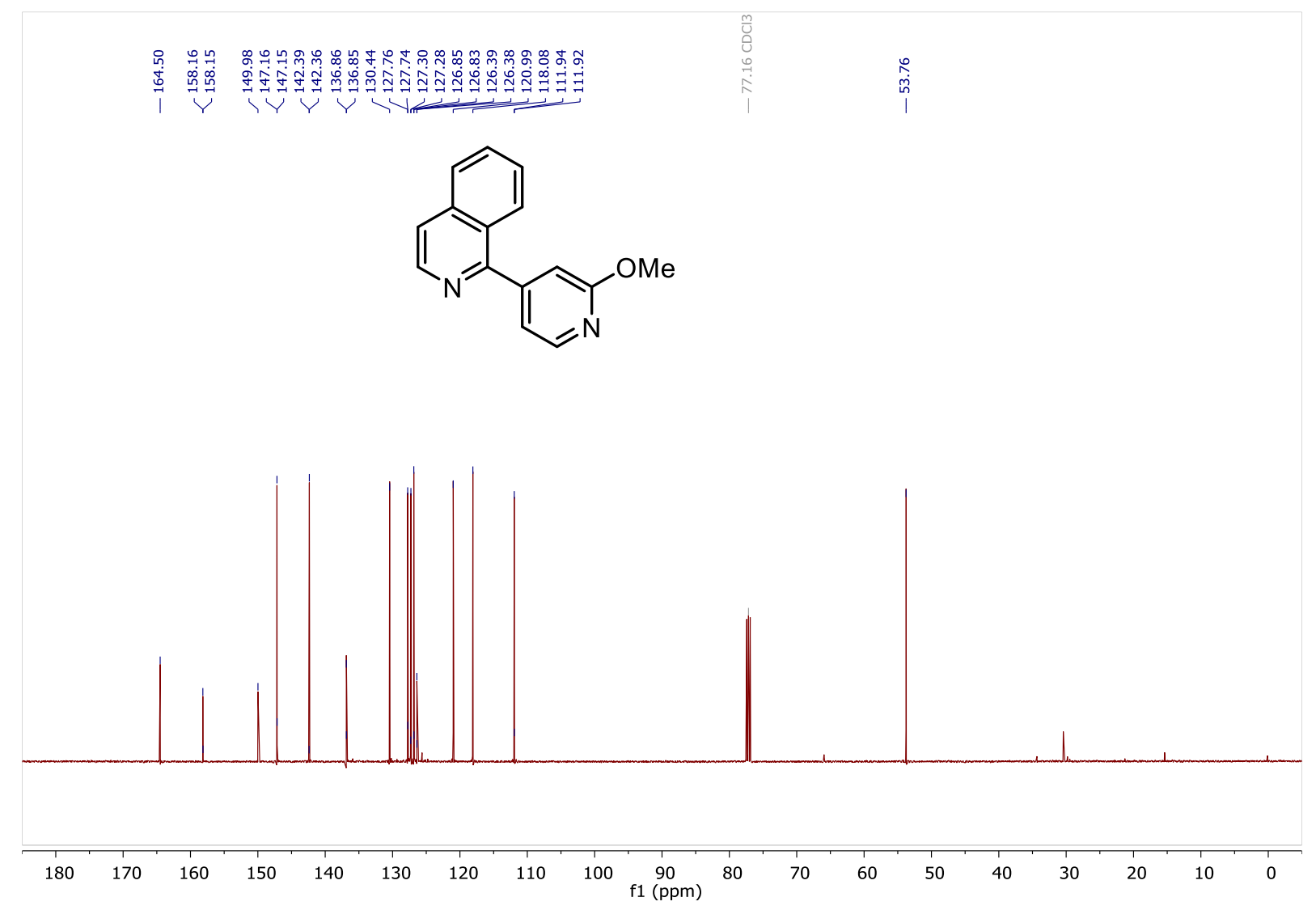


${ }^{1} \mathrm{H}$ NMR (500 MHz, $\mathrm{CDCl}_{3}$ ): 19

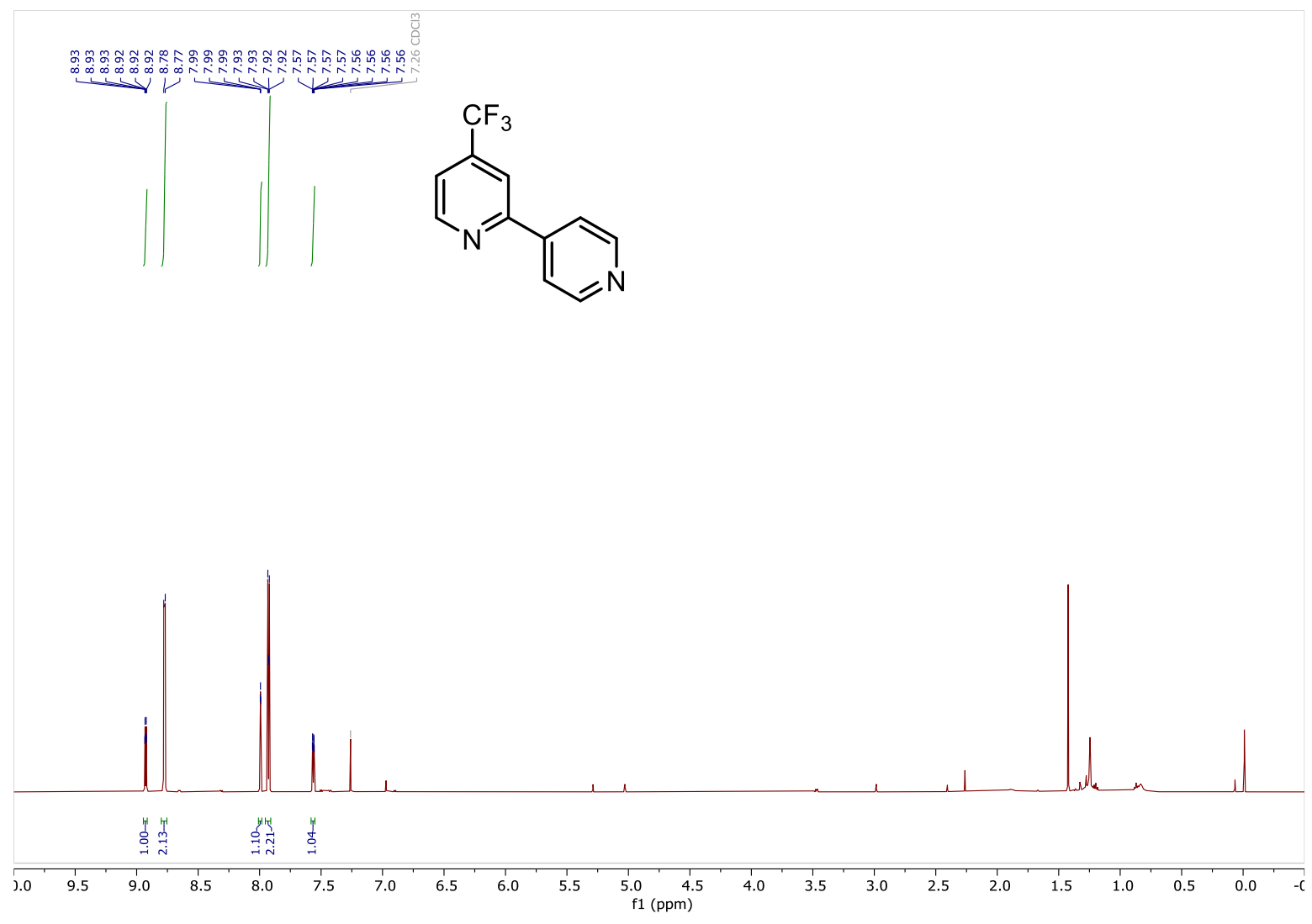

${ }^{13} \mathrm{C}$ NMR (126 MHz, $\left.\mathrm{CDCl}_{3}\right): 19$

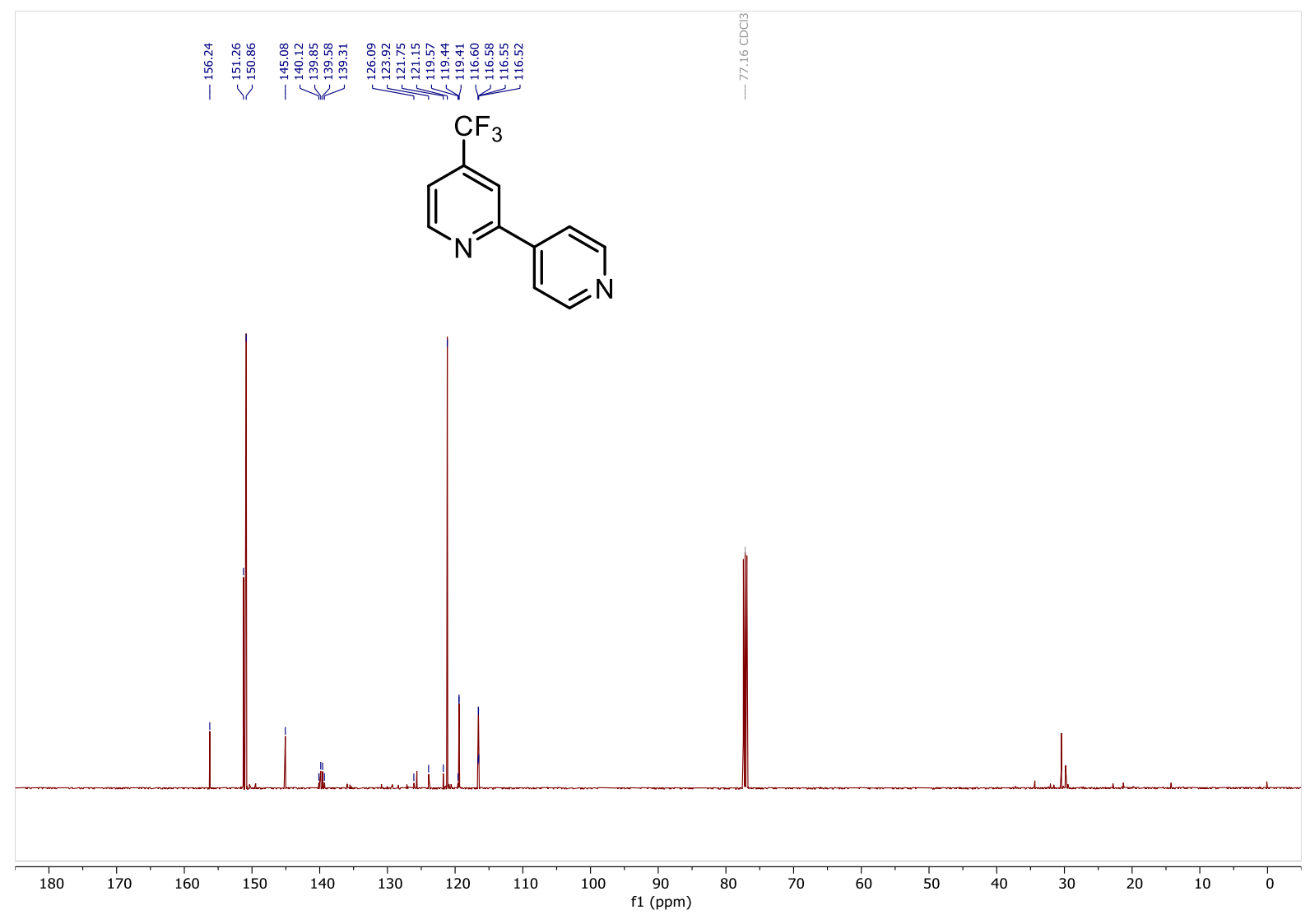

59 
${ }^{1} \mathrm{H}$ NMR (500 MHz, $\mathrm{CDCl}_{3}$ ): 20

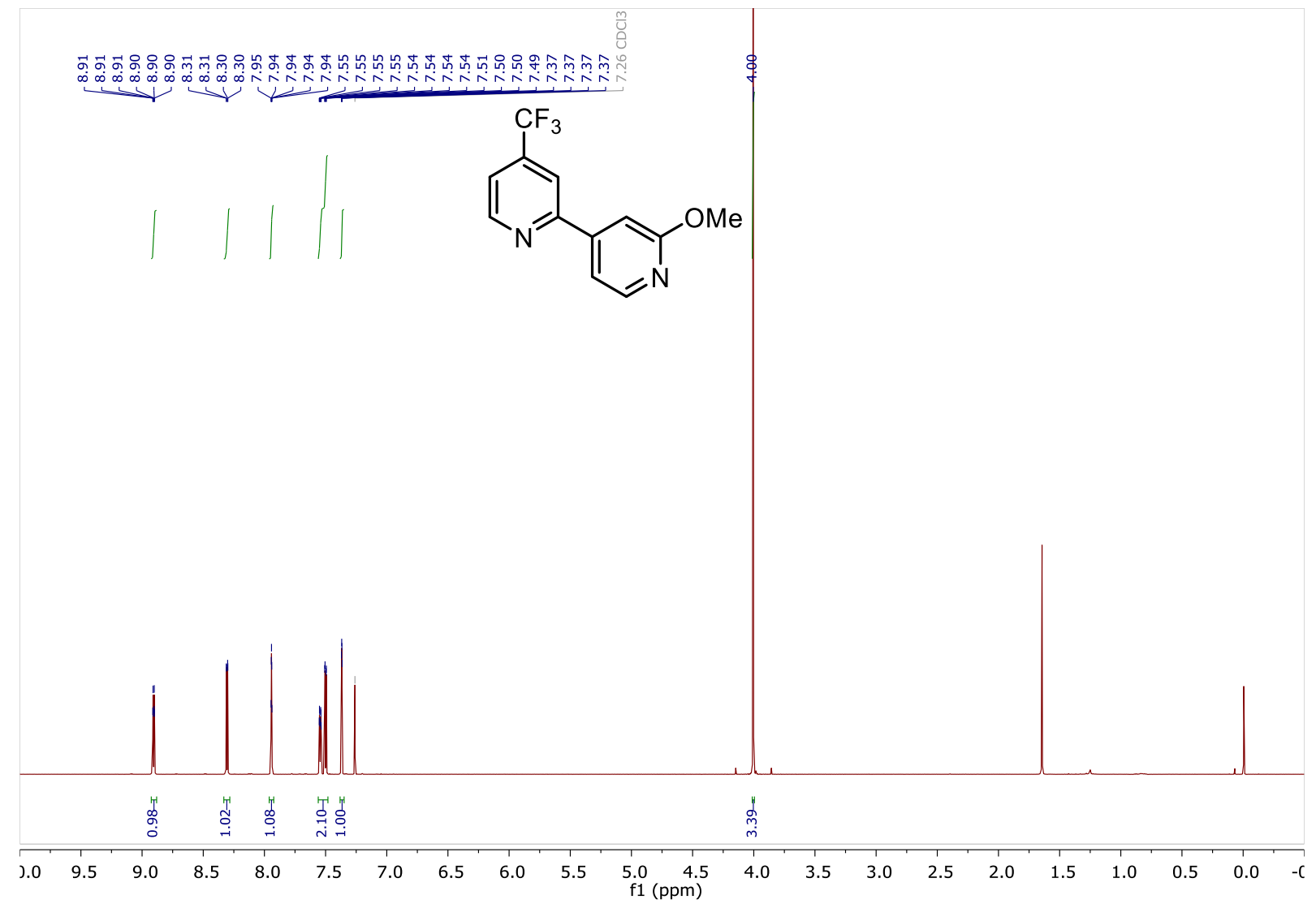

${ }^{13} \mathrm{C}$ NMR (126 MHz, $\left.\mathrm{CDCl}_{3}\right): 20$

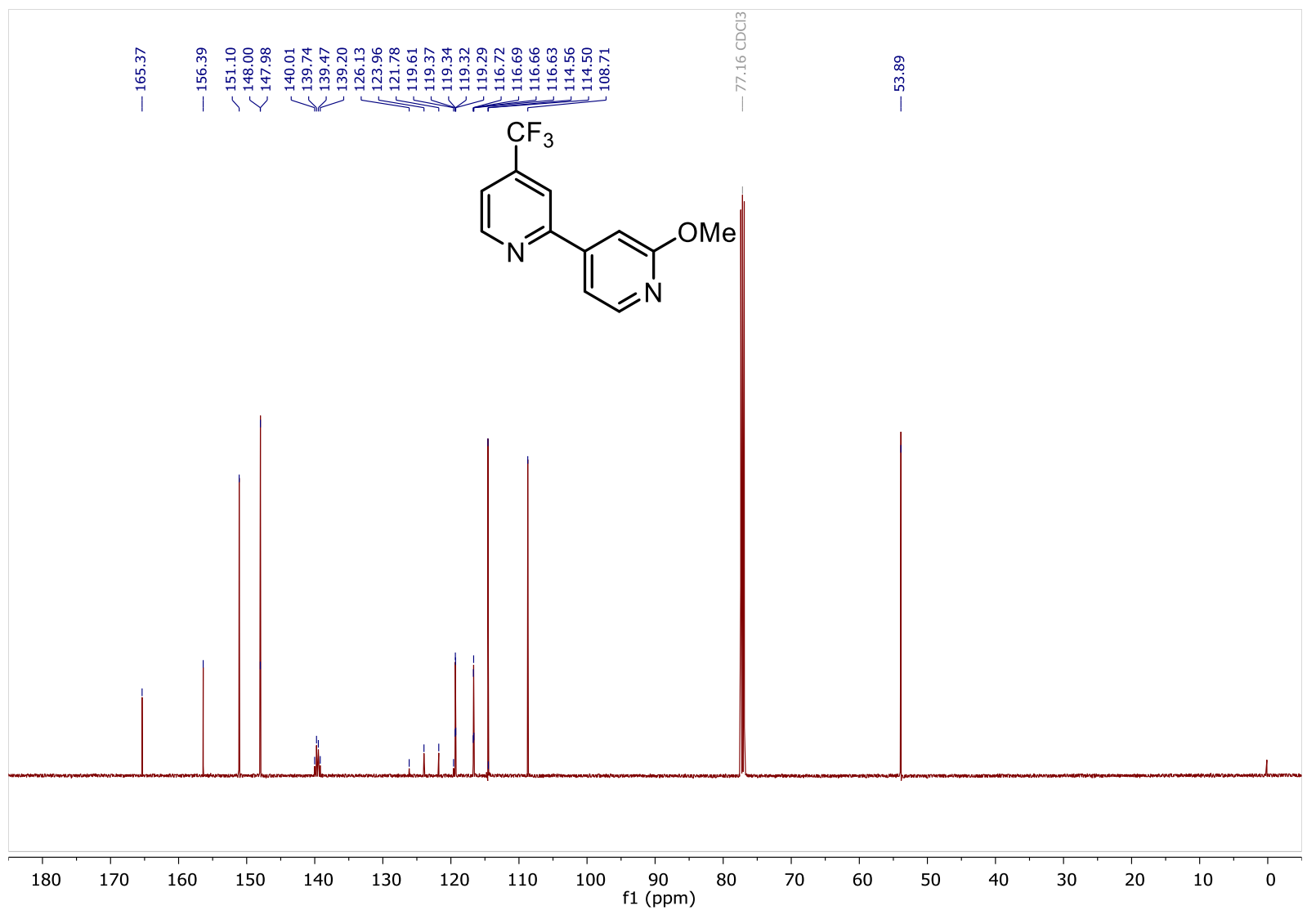


${ }^{1} \mathrm{H}$ NMR (500 MHz, $\mathrm{CDCl}_{3}$ ): 21

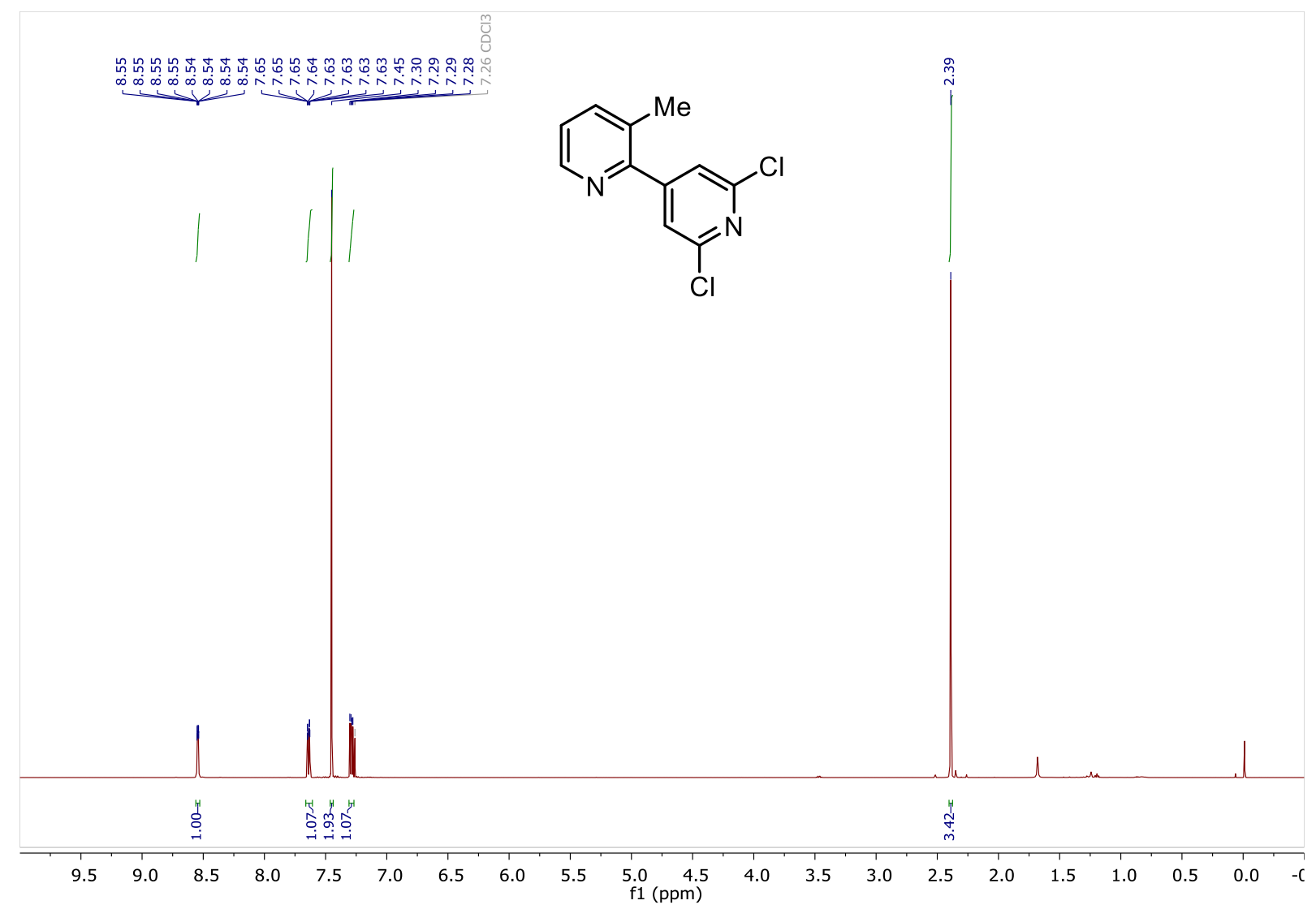

${ }^{13} \mathrm{C}$ NMR (126 MHz, $\left.\mathrm{CDCl}_{3}\right): 21$

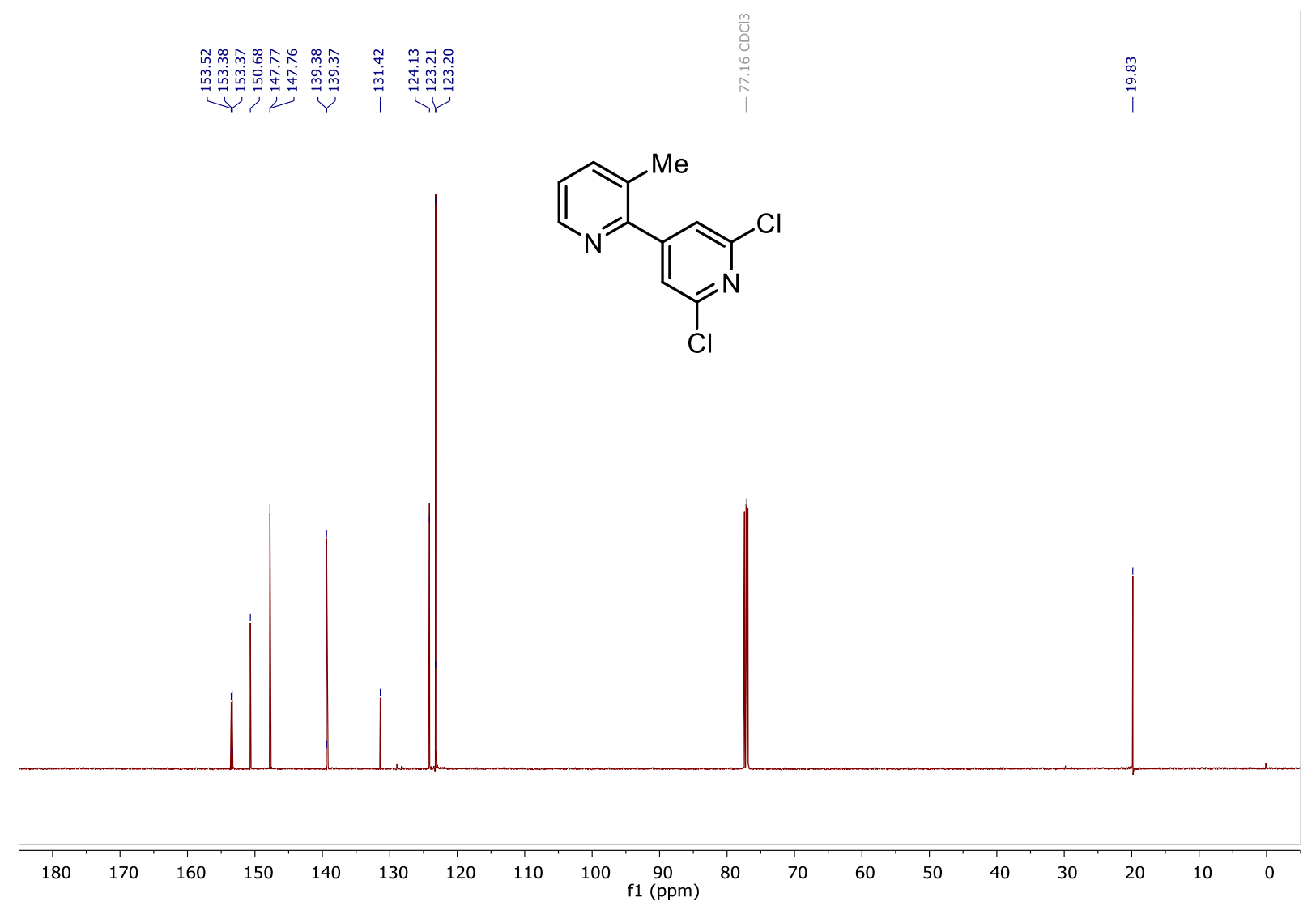


${ }^{1} \mathrm{H}$ NMR (500 MHz, $\mathrm{CDCl}_{3}$ ): 22

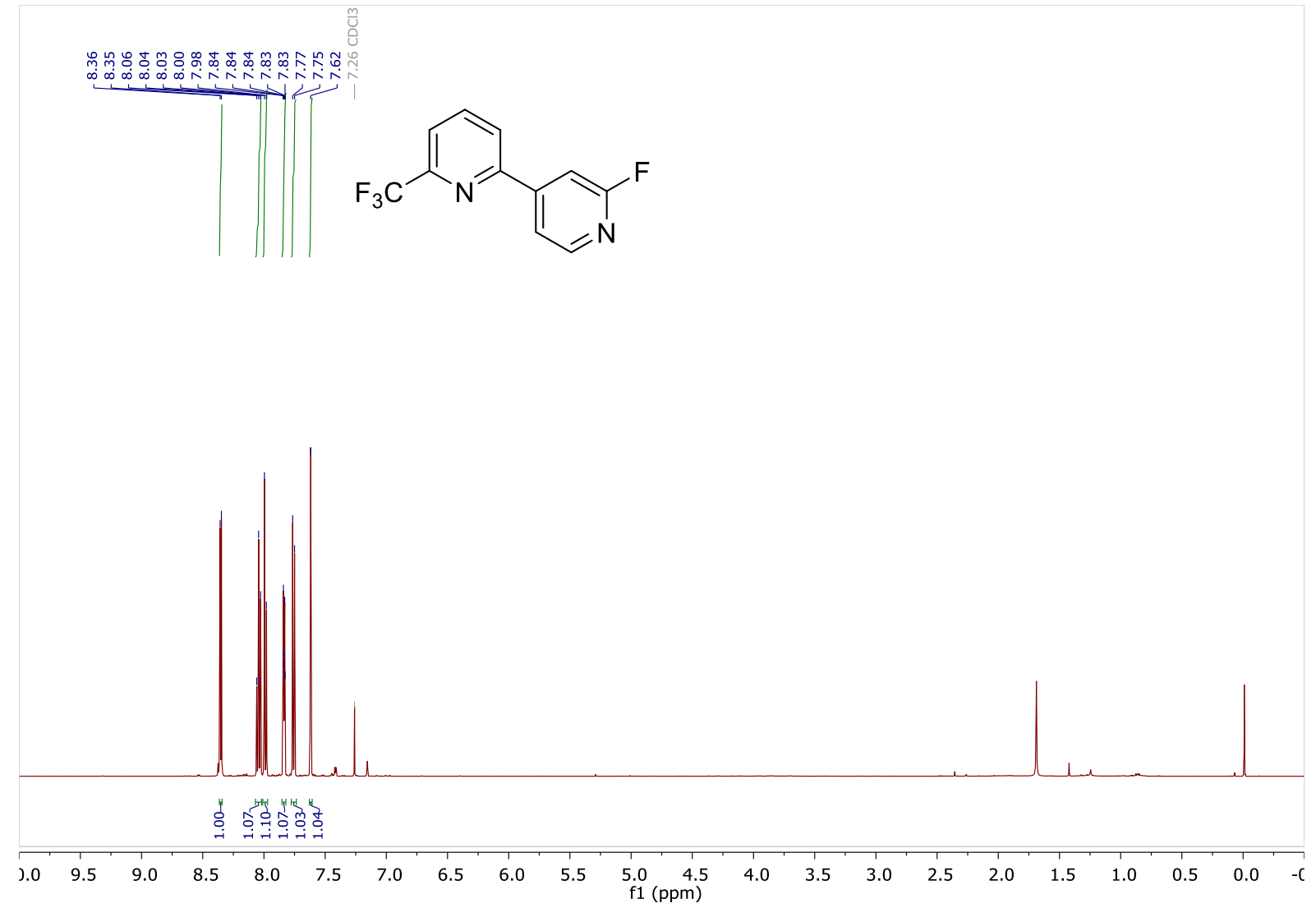

${ }^{13} \mathrm{C}$ NMR (126 MHz, $\left.\mathrm{CDCl}_{3}\right): 22$

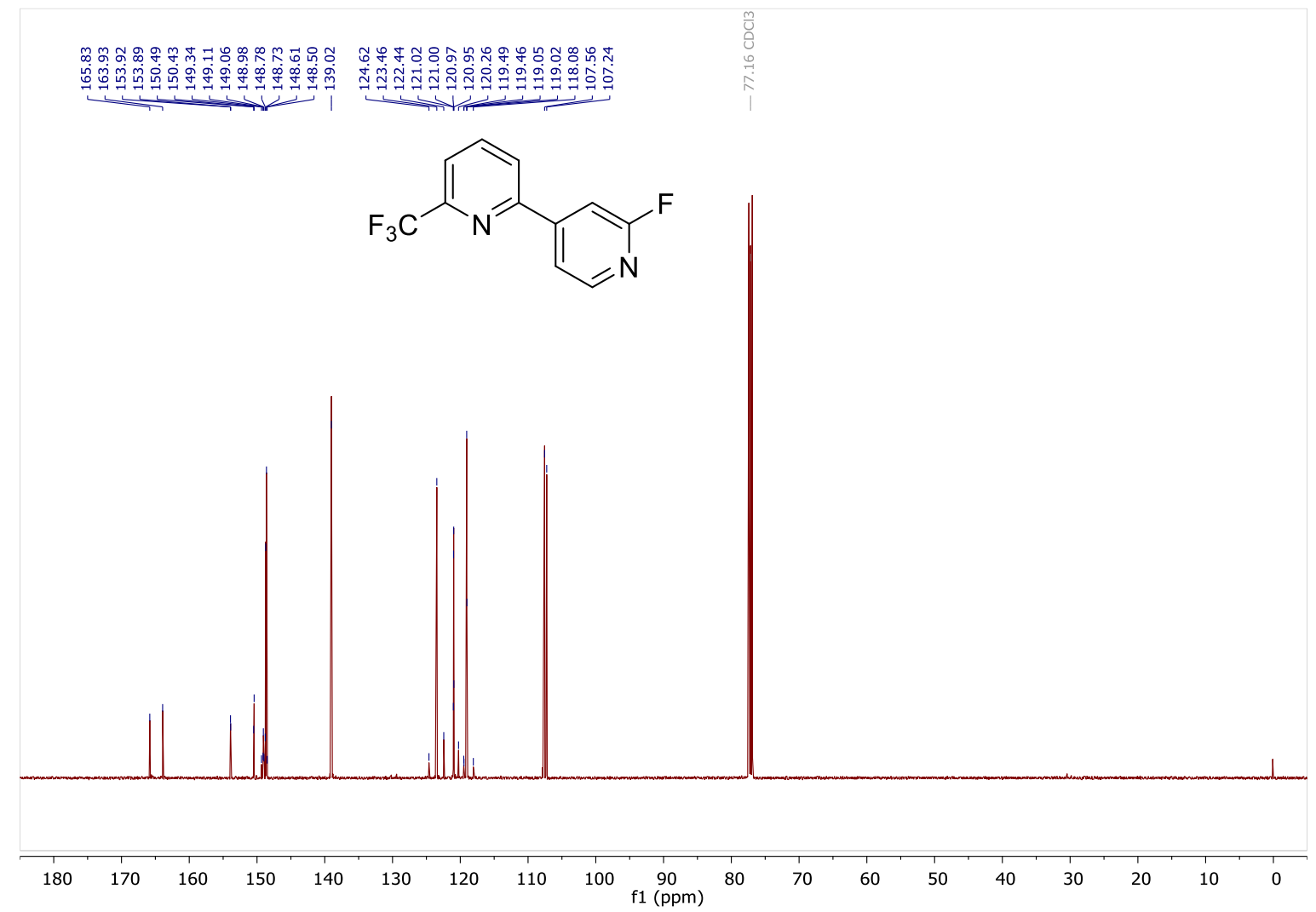


${ }^{1} \mathrm{H}$ NMR (400 MHz, $\mathrm{CDCl}_{3}$ ): 23

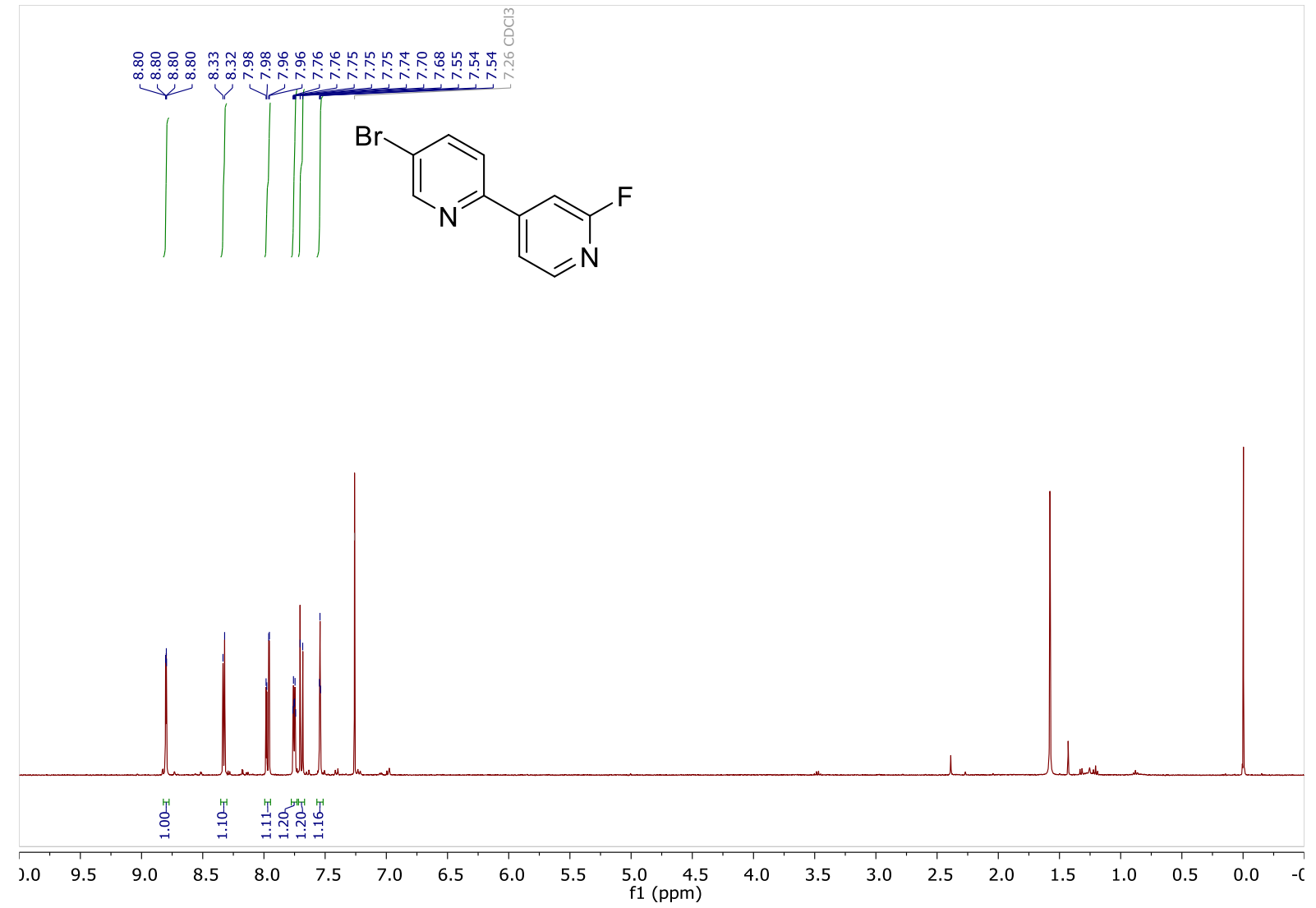

${ }^{13}$ C NMR (126 MHz, $\left.\mathrm{CDCl}_{3}\right): 23$

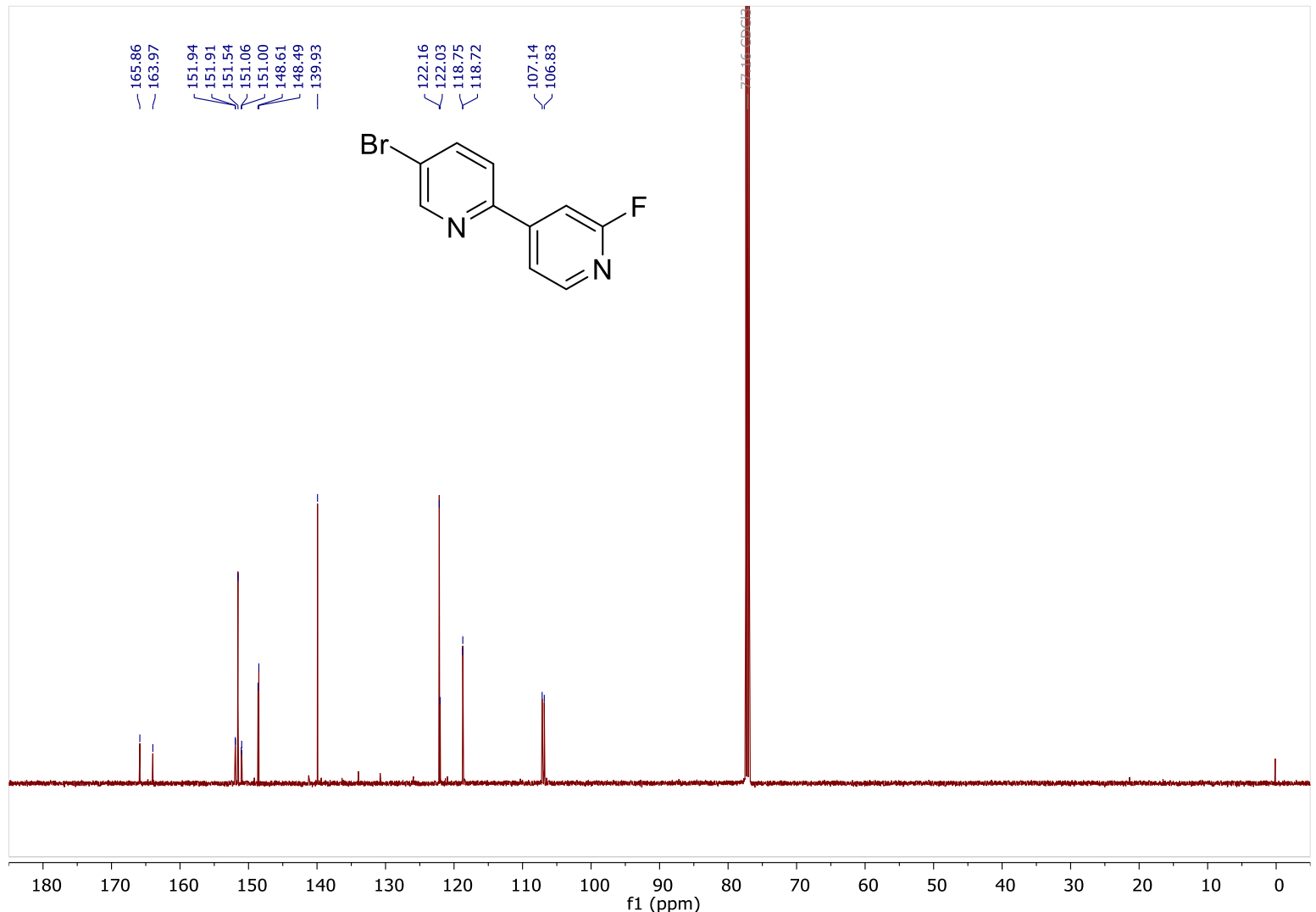


${ }^{1} \mathrm{H}$ NMR (400 MHz, $\mathrm{CDCl}_{3}$ ): 24

$\underbrace{0}$

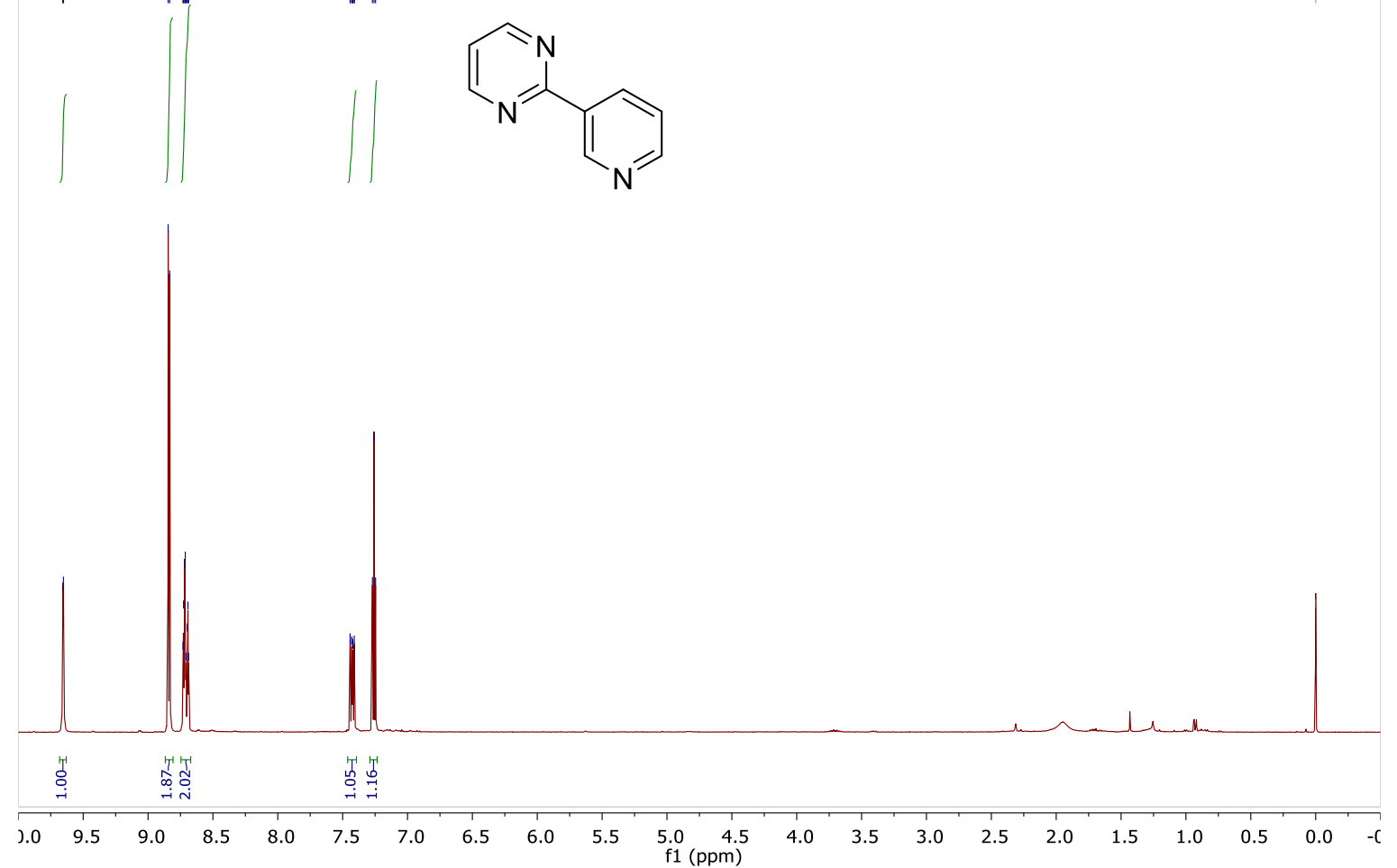

${ }^{13}$ C NMR (101 MHz, $\left.\mathrm{CDCl}_{3}\right): 24$

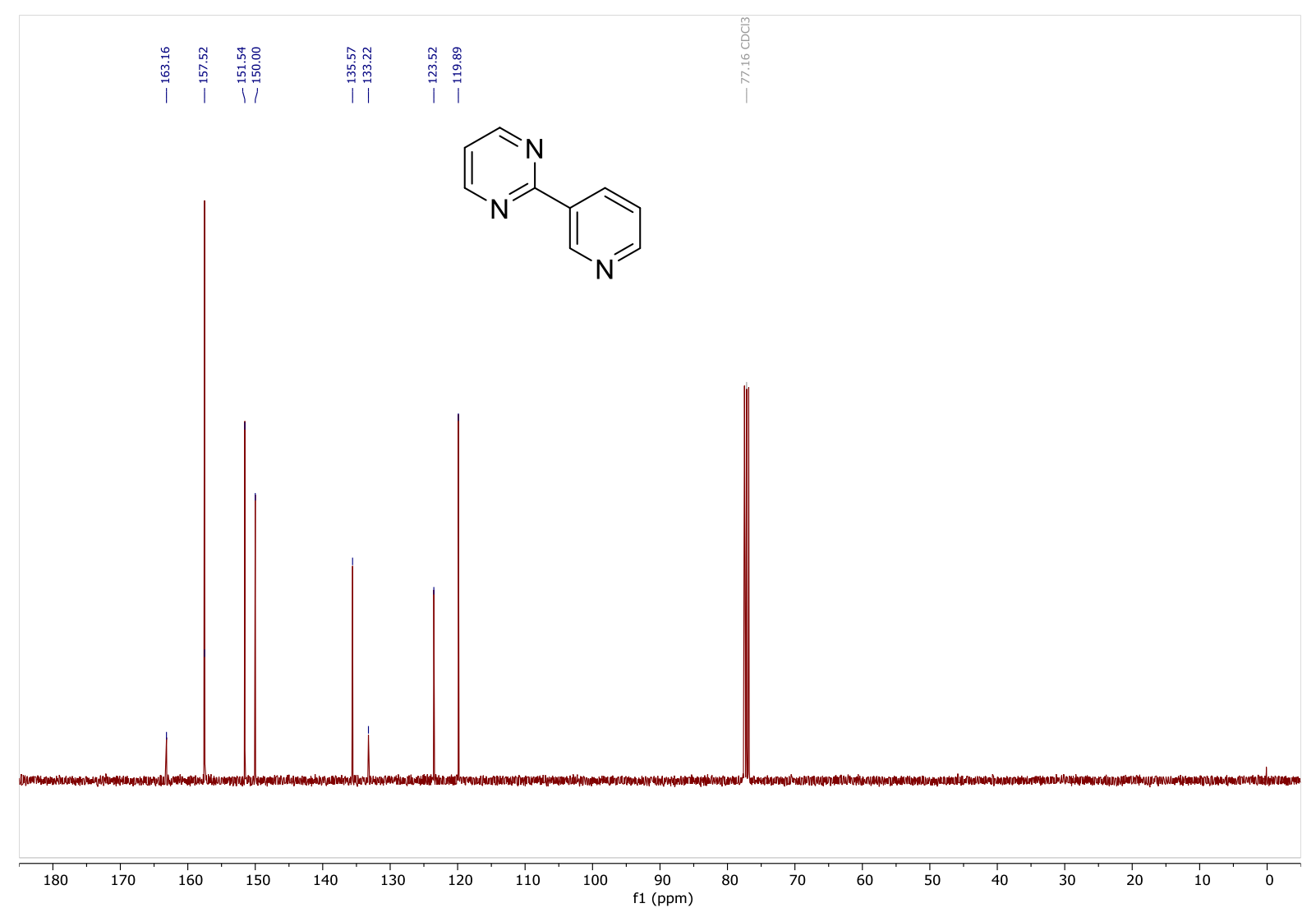


${ }^{1} \mathrm{H}$ NMR (400 MHz, $\mathrm{CDCl}_{3}$ ): 25

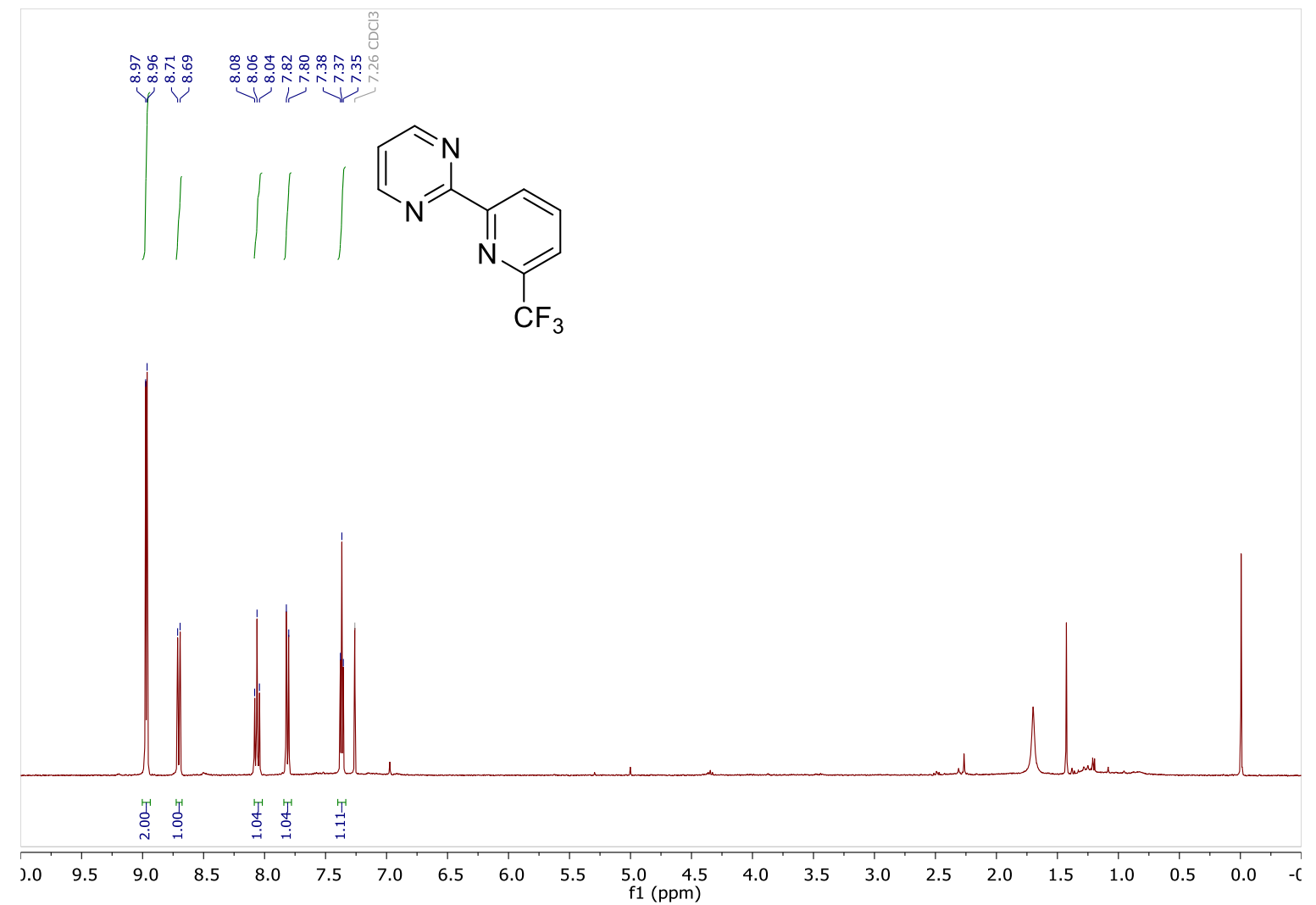

${ }^{13} \mathrm{C}$ NMR (101 MHz, $\left.\mathrm{CDCl}_{3}\right): 25$

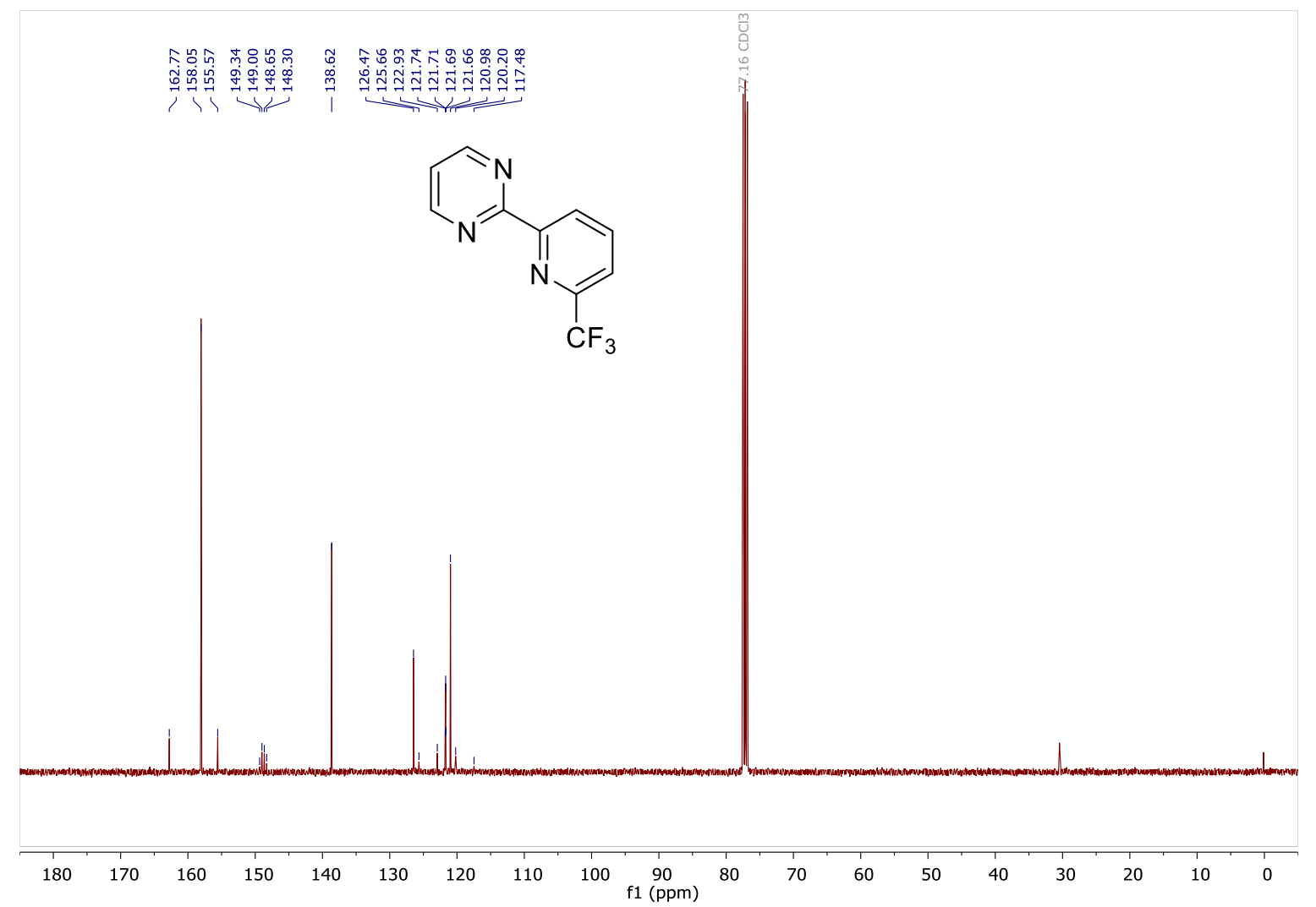


${ }^{1} \mathrm{H}$ NMR (500 MHz, $\mathrm{CDCl}_{3}$ ): 26

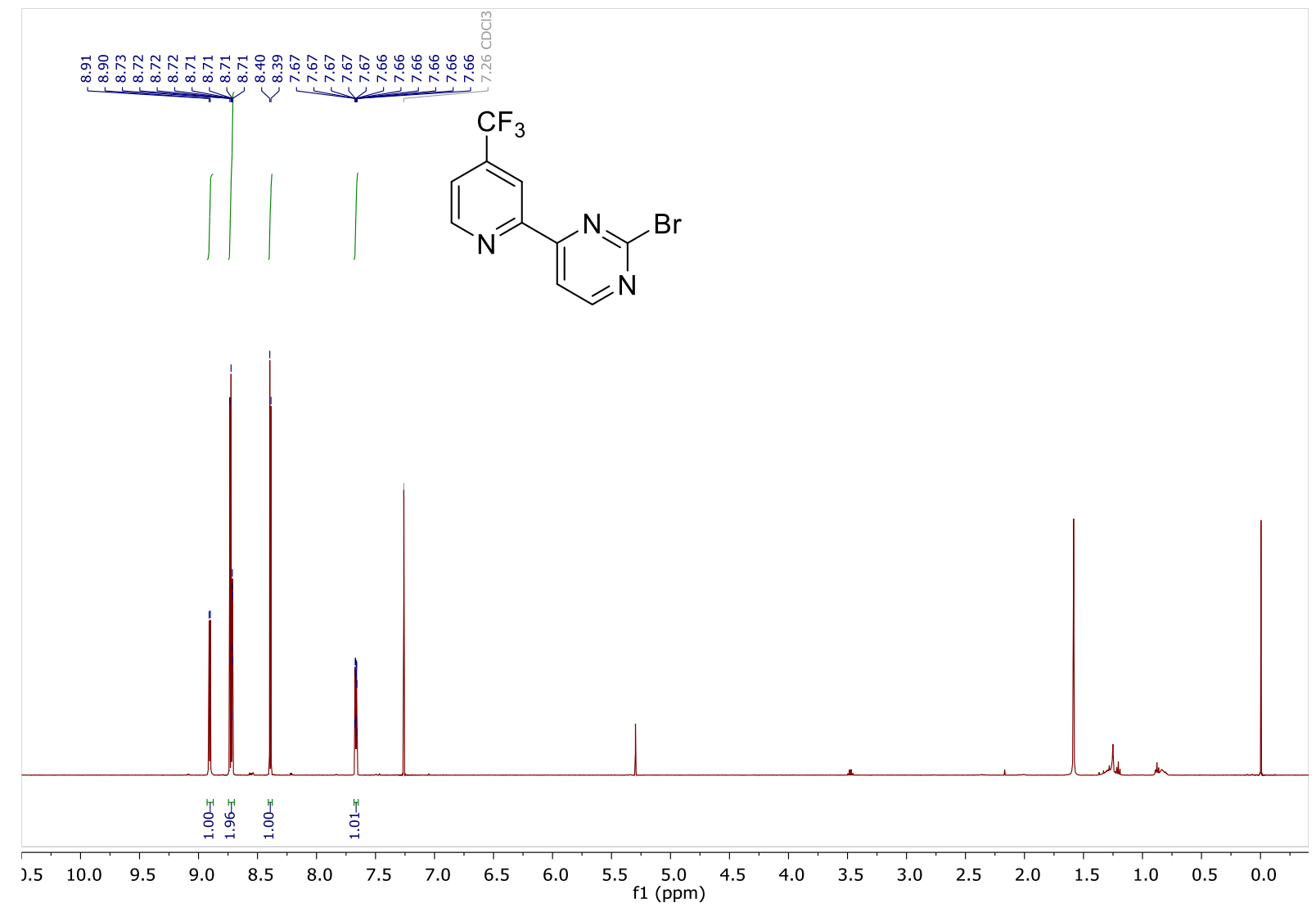

${ }^{13}$ C NMR (101 MHz, $\left.\mathrm{CDCl}_{3}\right): 26$

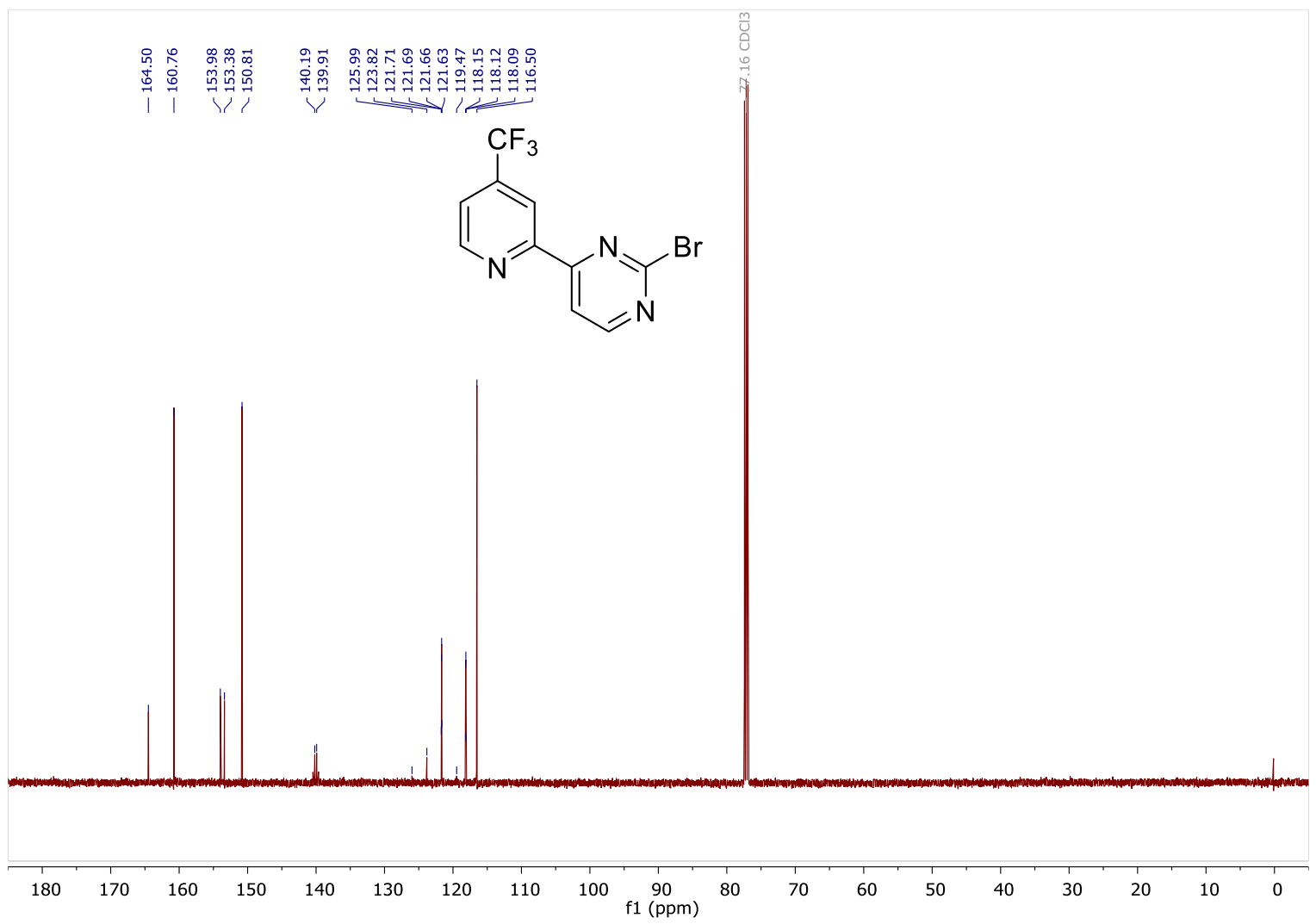


${ }^{1} \mathrm{H}$ NMR (400 MHz, $\mathrm{CDCl}_{3}$ ): 27

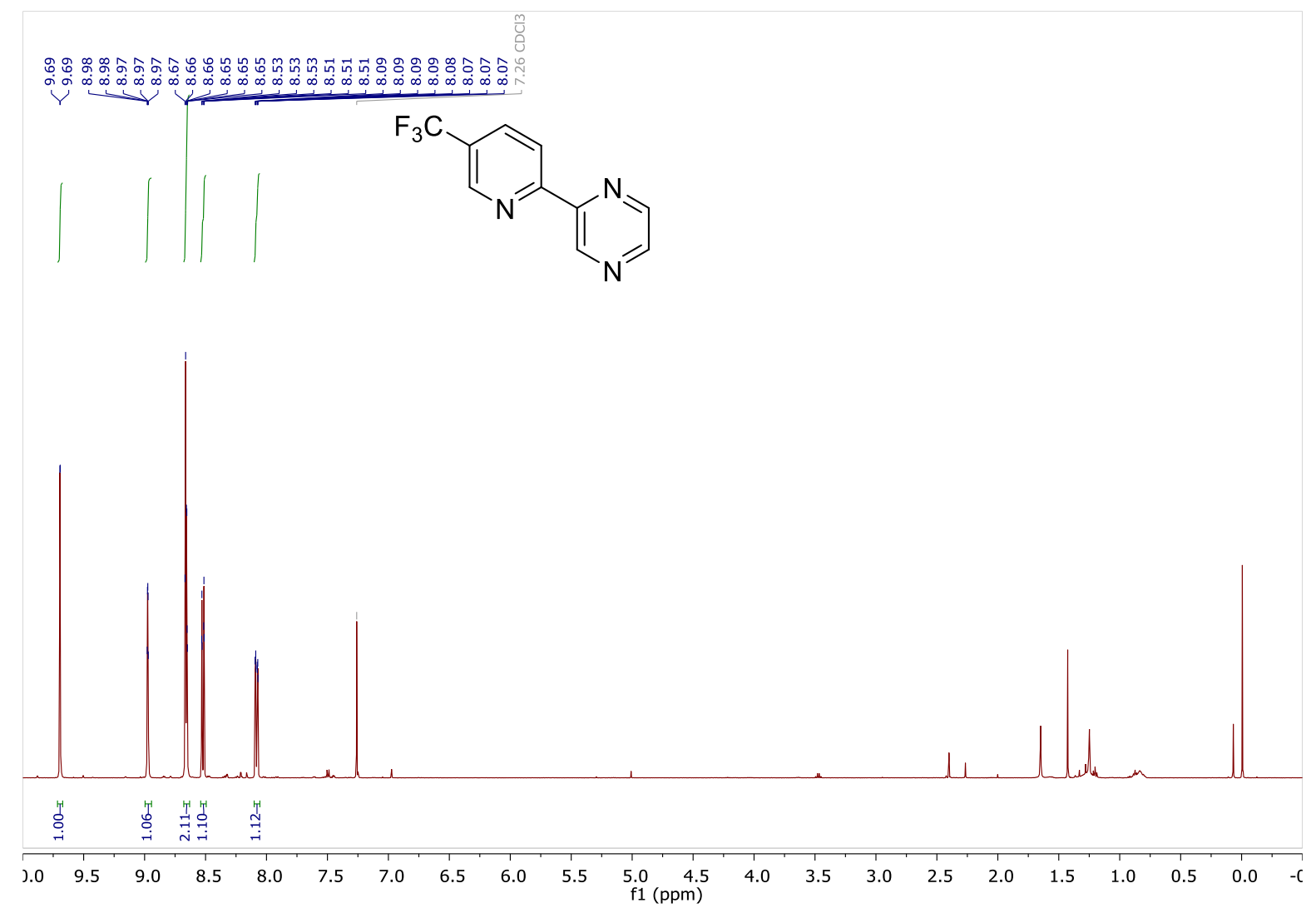

${ }^{13}$ C NMR (101 MHz, $\left.\mathrm{CDCl}_{3}\right): 27$

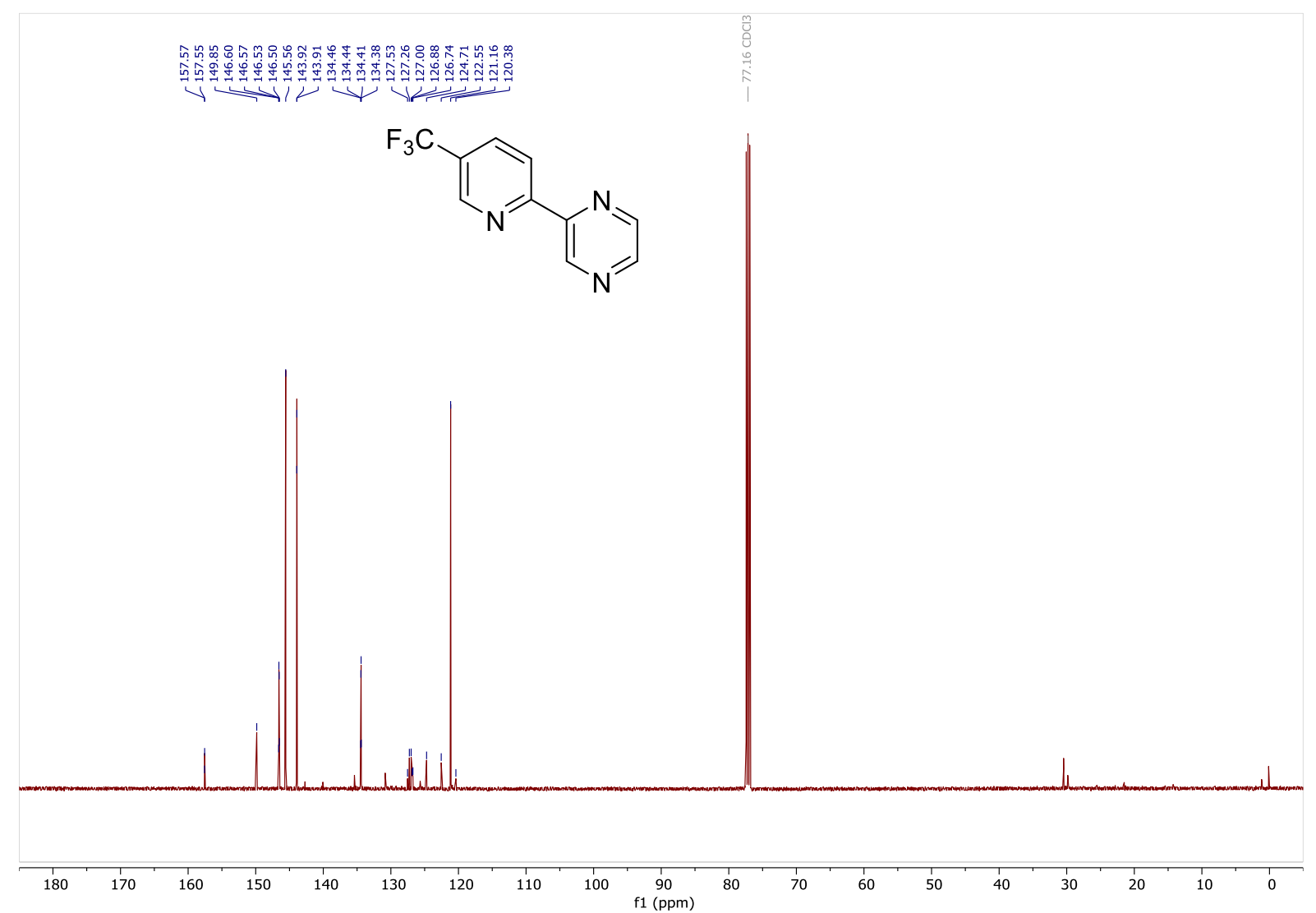


${ }^{1} \mathrm{H}$ NMR (400 MHz, $\mathrm{CDCl}_{3}$ ): 28

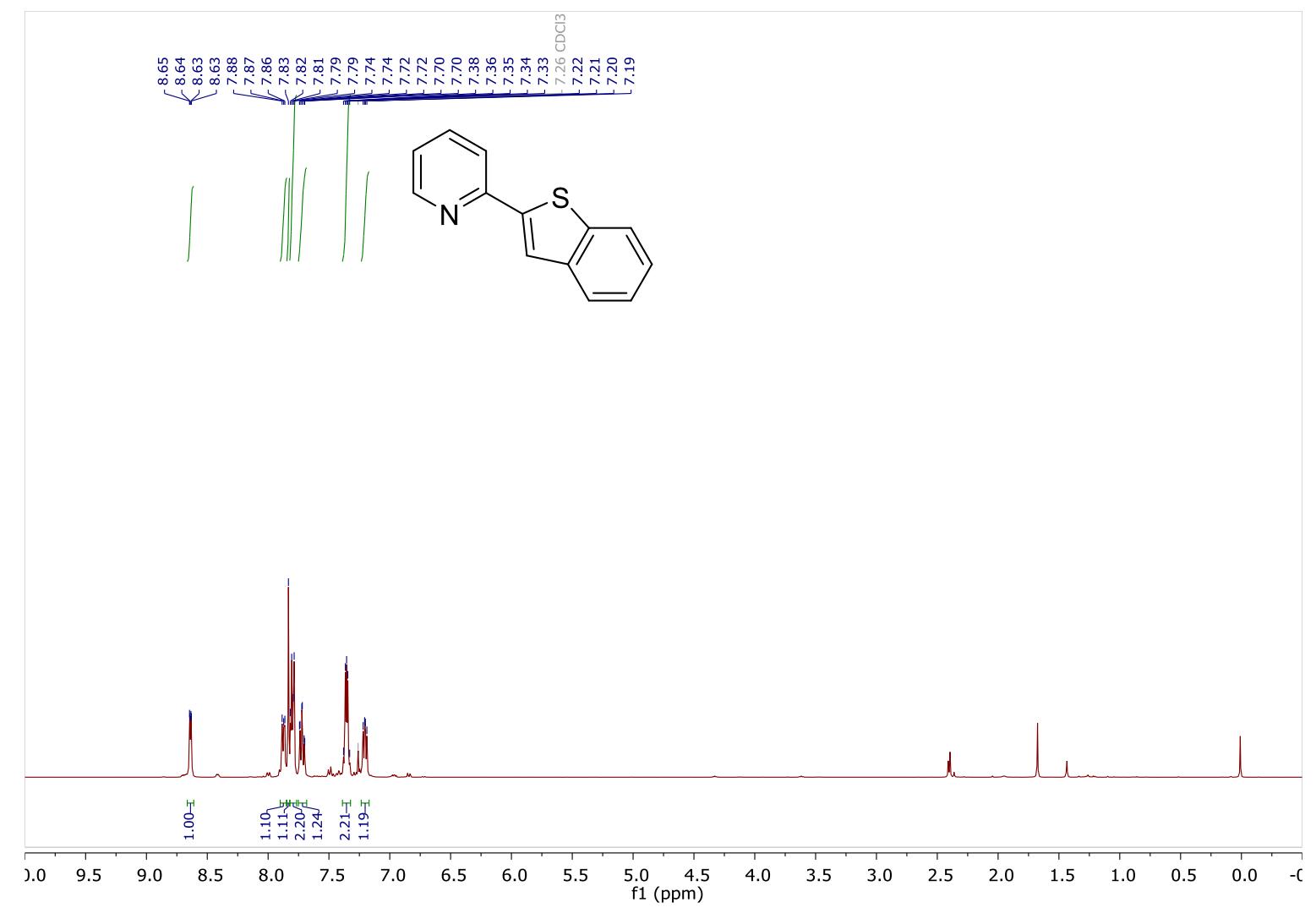

${ }^{13} \mathrm{C}$ NMR (101 MHz, $\left.\mathrm{CDCl}_{3}\right): 28$

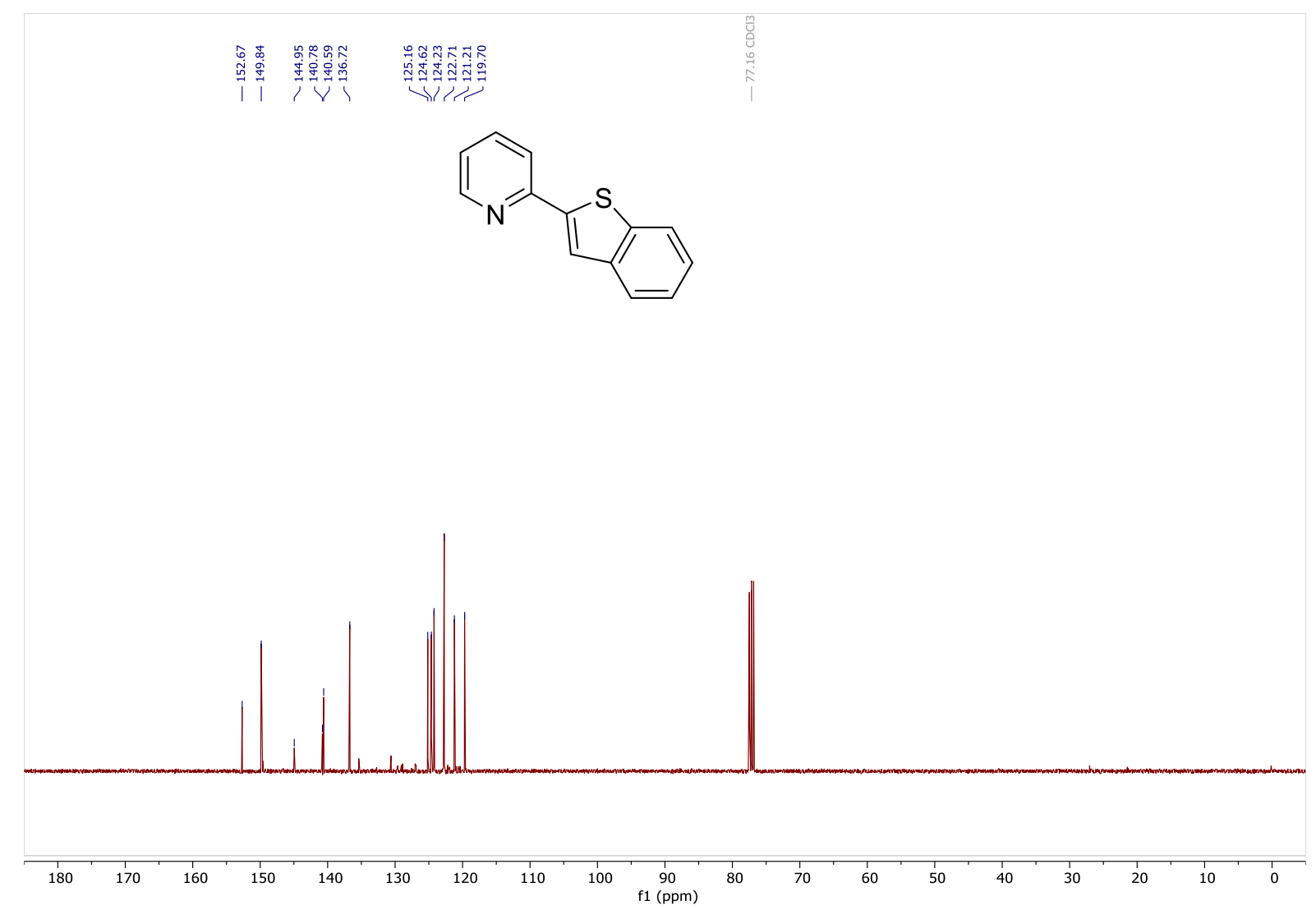


${ }^{1} \mathrm{H}$ NMR (400 MHz, $\mathrm{CDCl}_{3}$ ): 29

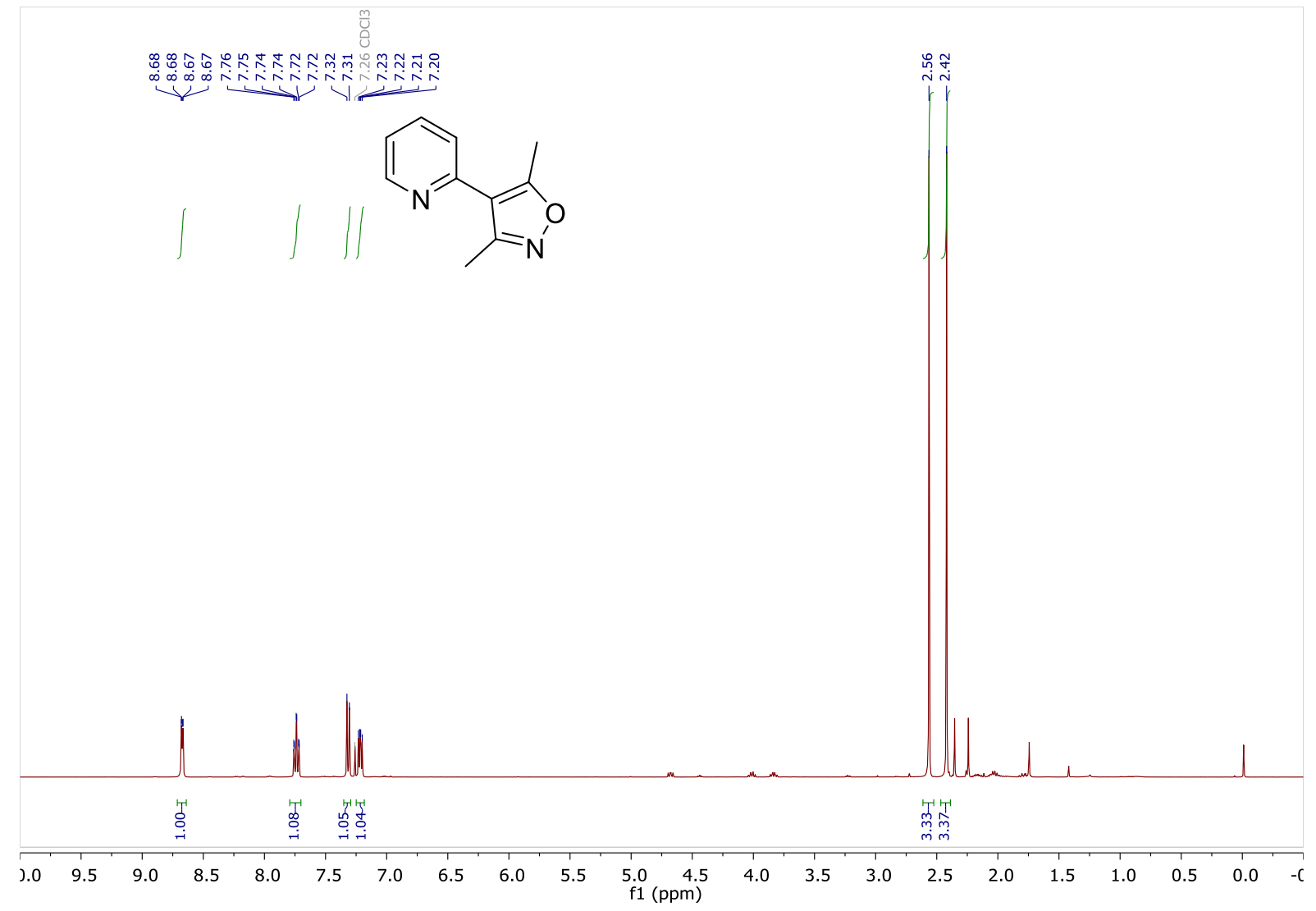

${ }^{13} \mathrm{C}$ NMR (101 MHz, $\left.\mathrm{CDCl}_{3}\right): 29$

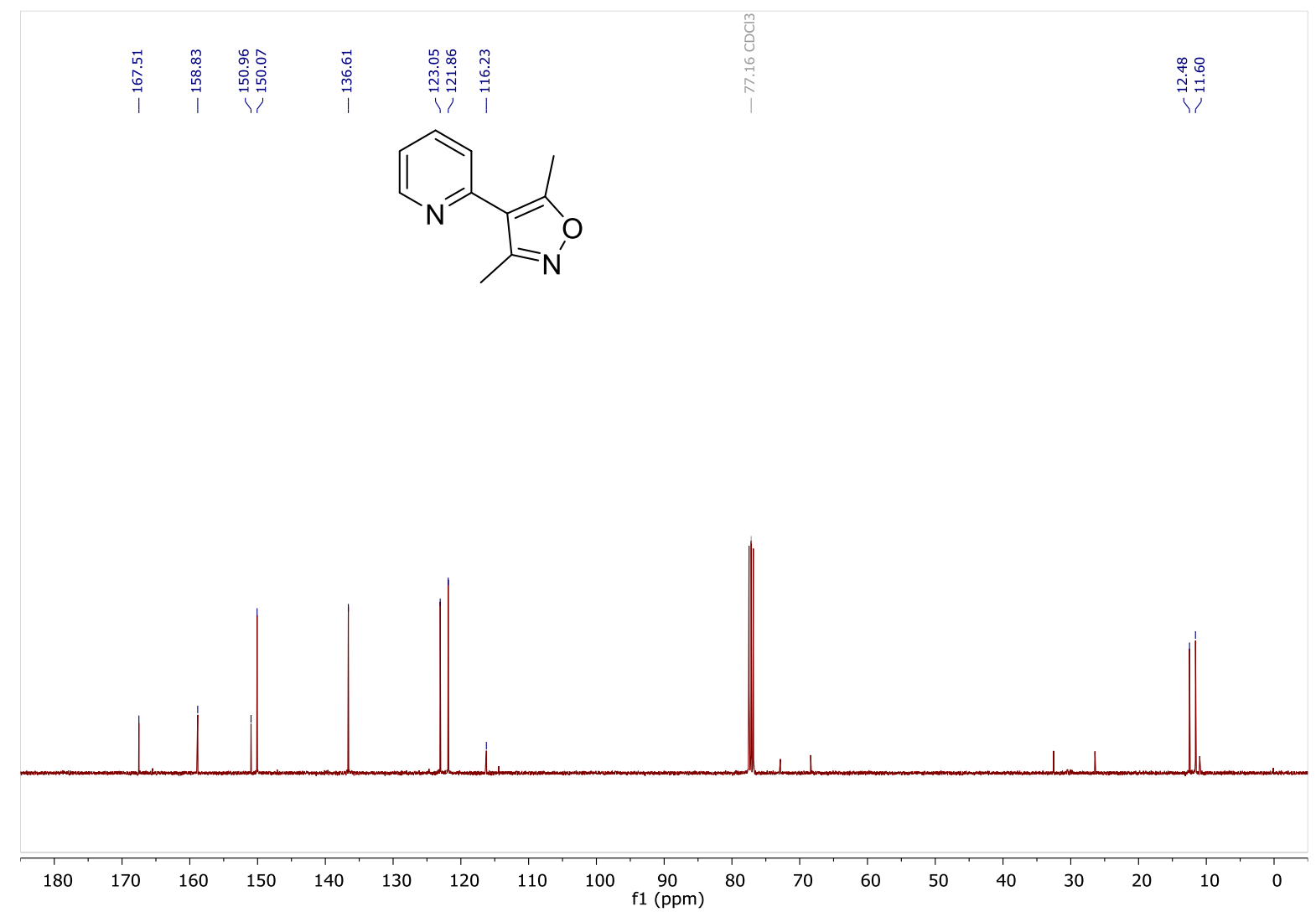


${ }^{1} \mathrm{H}$ NMR (400 MHz, $\mathrm{CDCl}_{3}$ ): 30

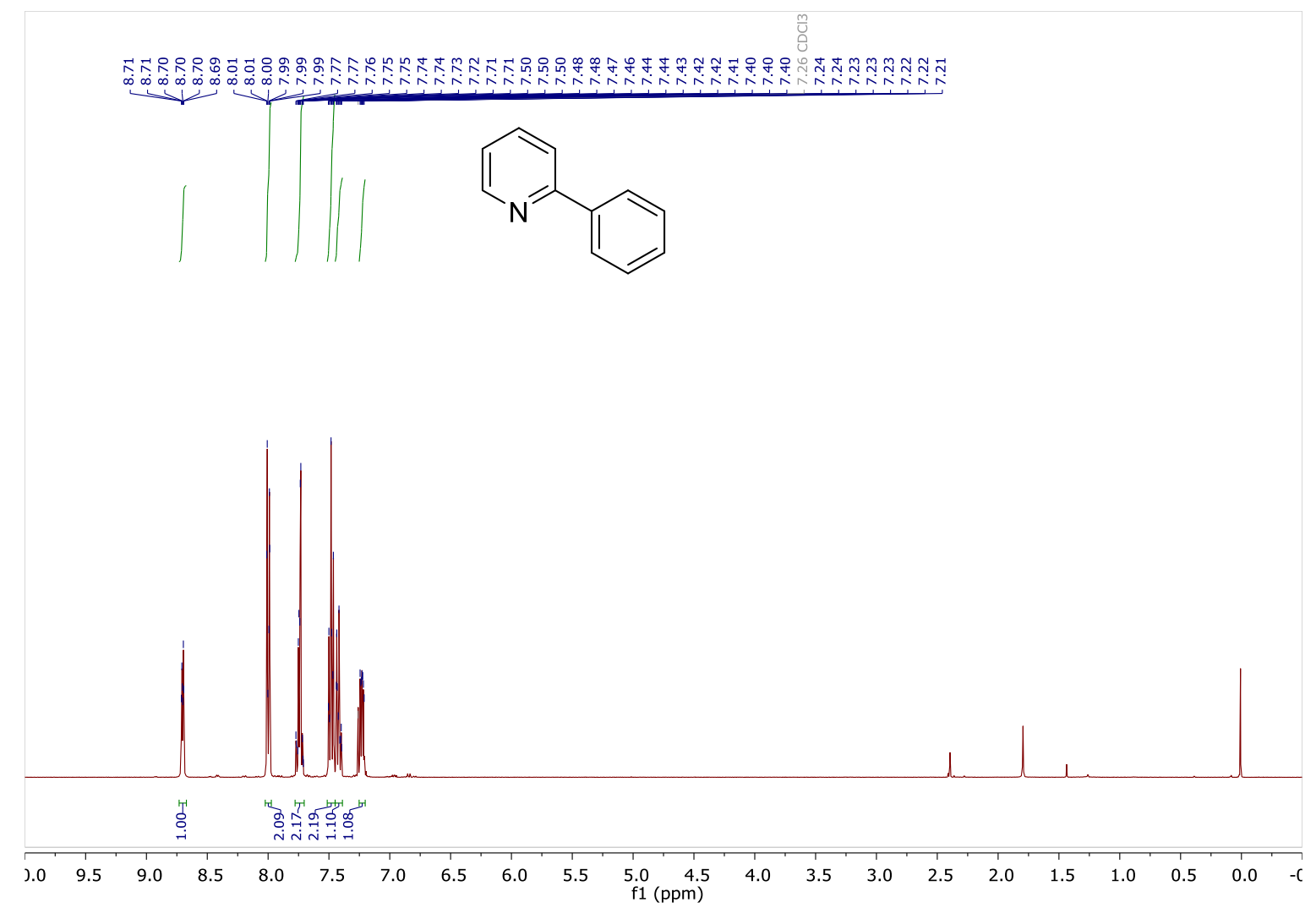

${ }^{13} \mathrm{C}$ NMR (101 MHz, $\left.\mathrm{CDCl}_{3}\right): 30$

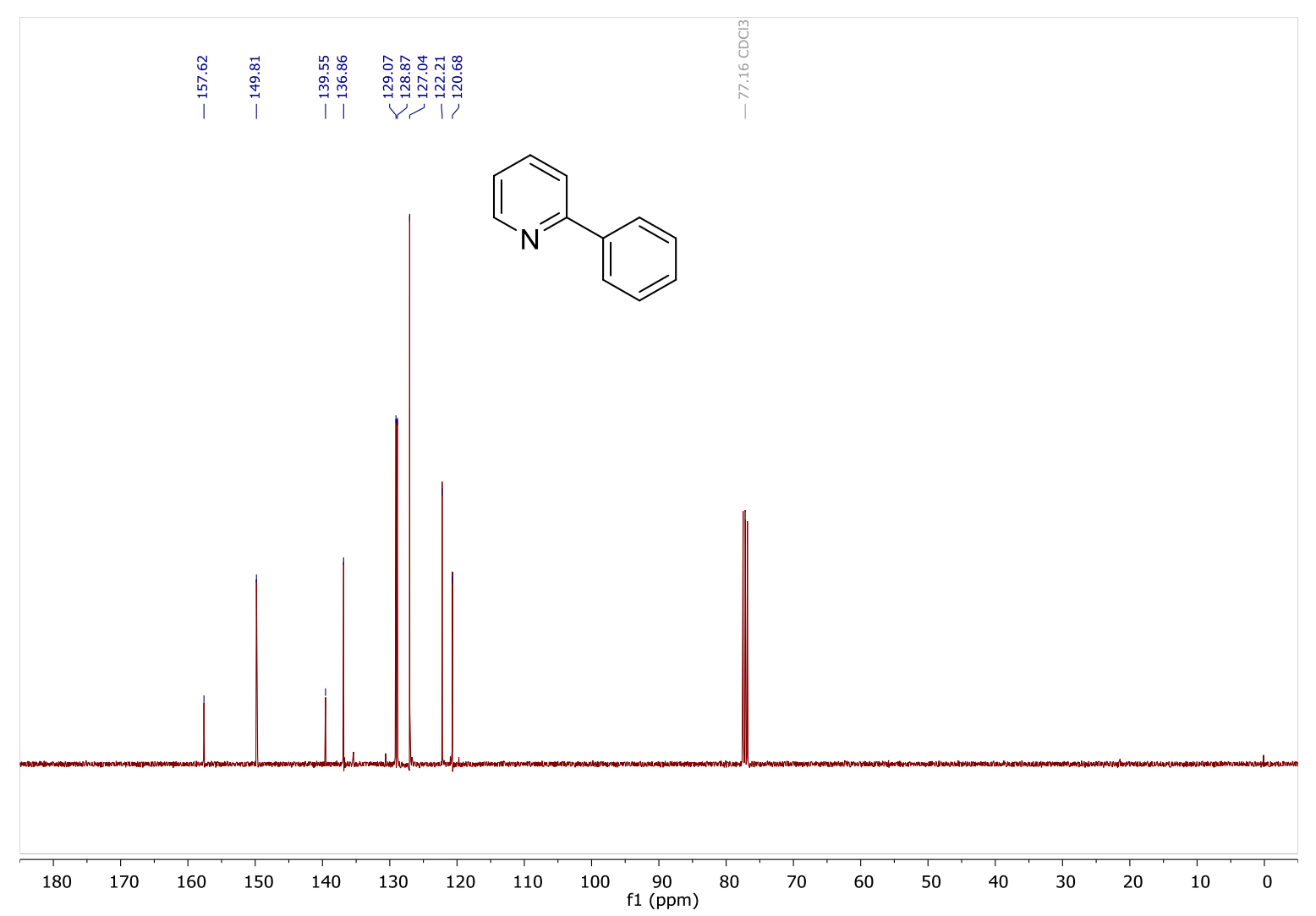


${ }^{1} \mathrm{H}$ NMR (400 MHz, $\mathrm{CDCl}_{3}$ ): 36

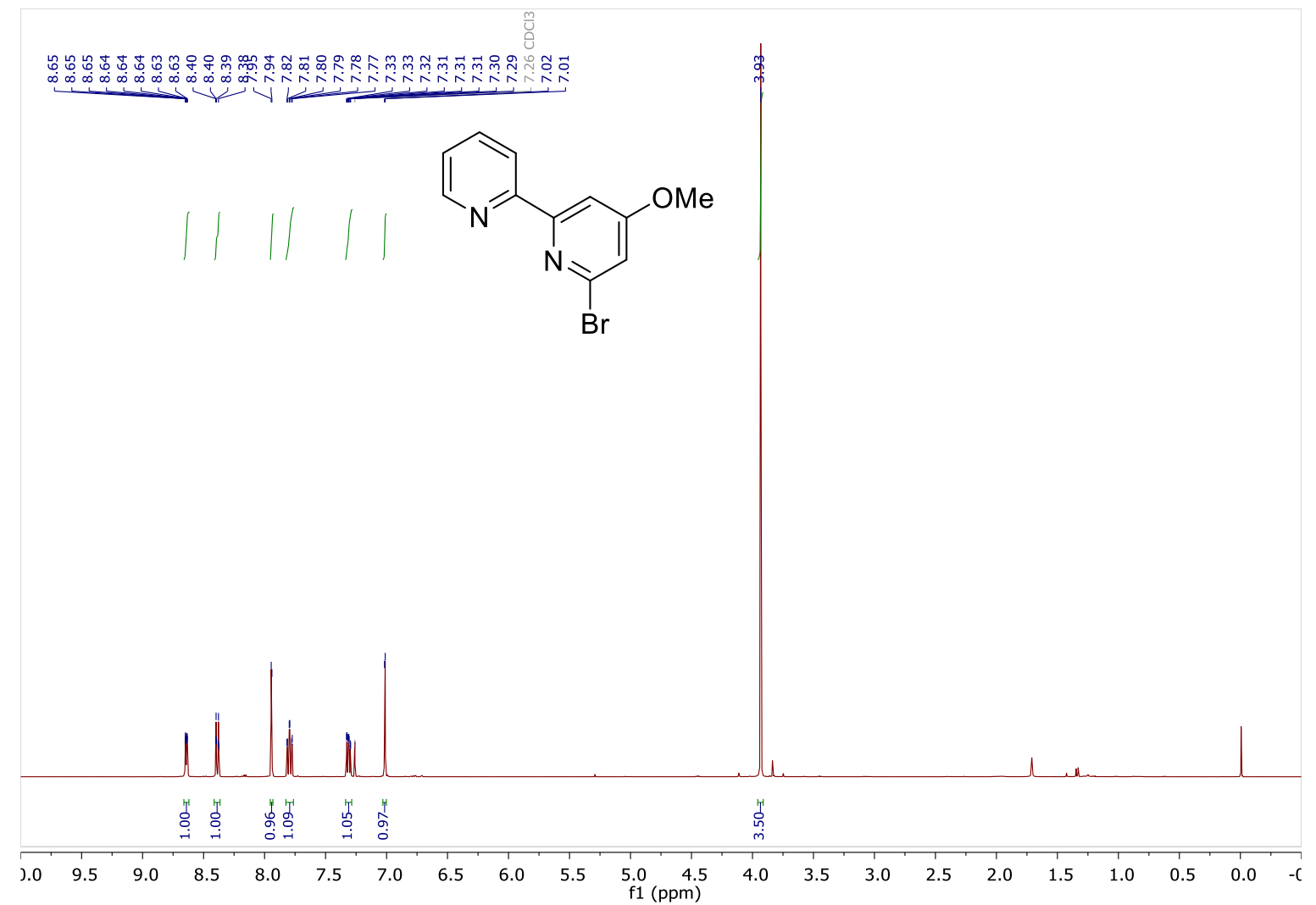

${ }^{13} \mathrm{C}$ NMR (101 MHz, $\left.\mathrm{CDCl}_{3}\right): 36$

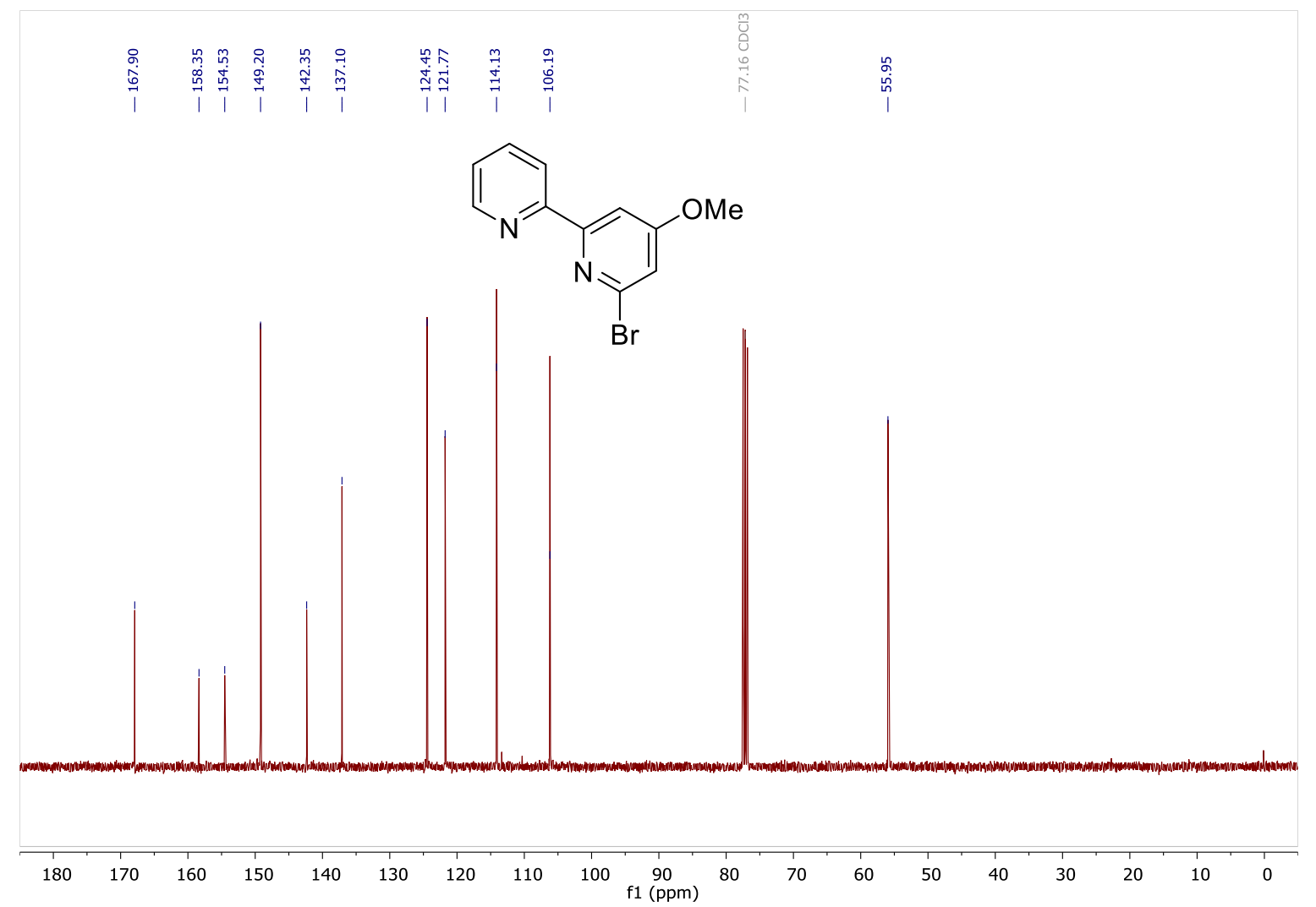


${ }^{1} \mathrm{H}$ NMR (400 MHz, $\mathrm{CDCl}_{3}$ ): 37

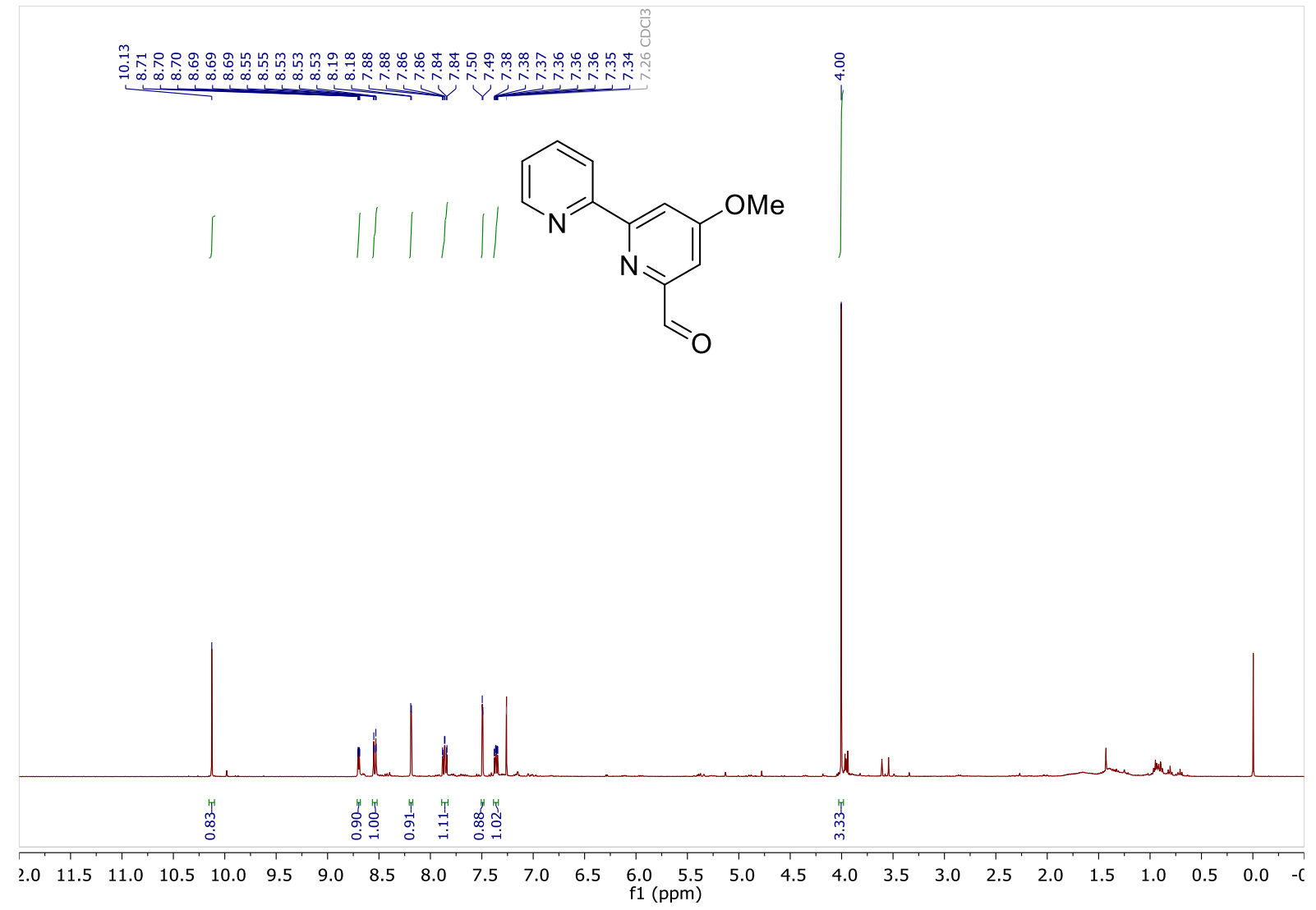

${ }^{13}$ C NMR (101 MHz, $\left.\mathrm{CDCl}_{3}\right): 37$

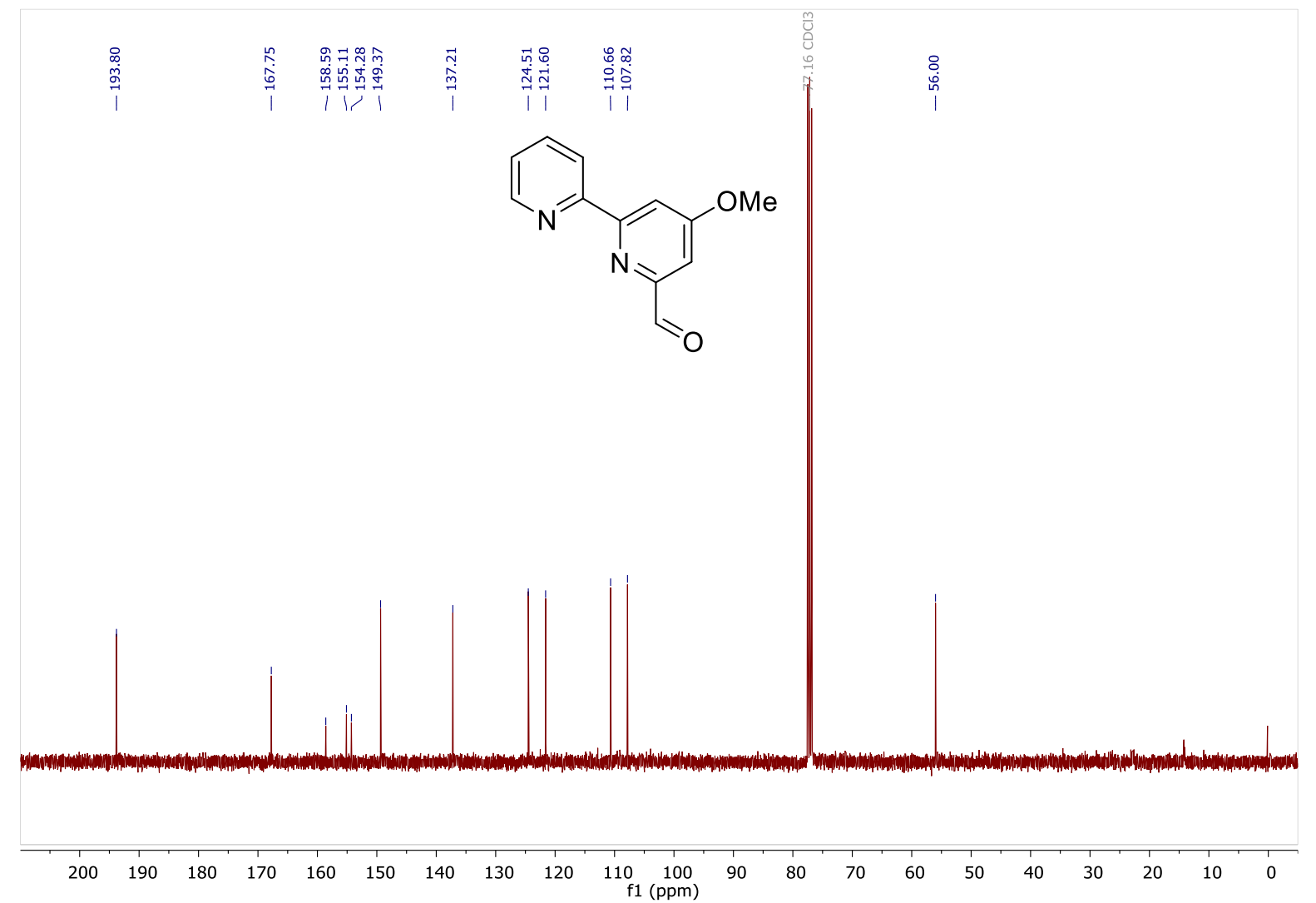


${ }^{1}$ H NMR (400 MHz, DMSO- $d_{6}$ ): 38

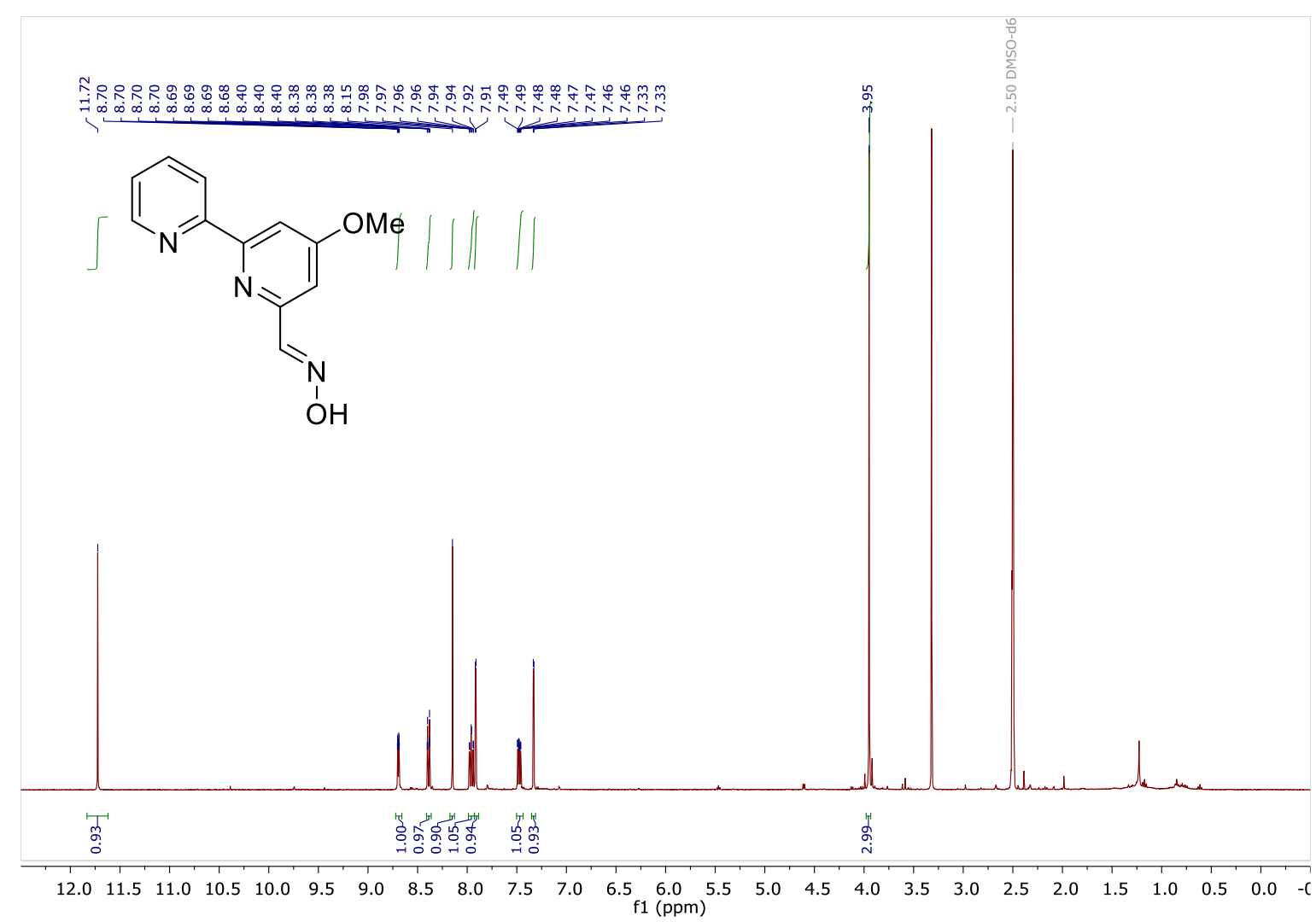

${ }^{13}$ C NMR (101 MHz, DMSO- $\left.d_{6}\right)$ : 38

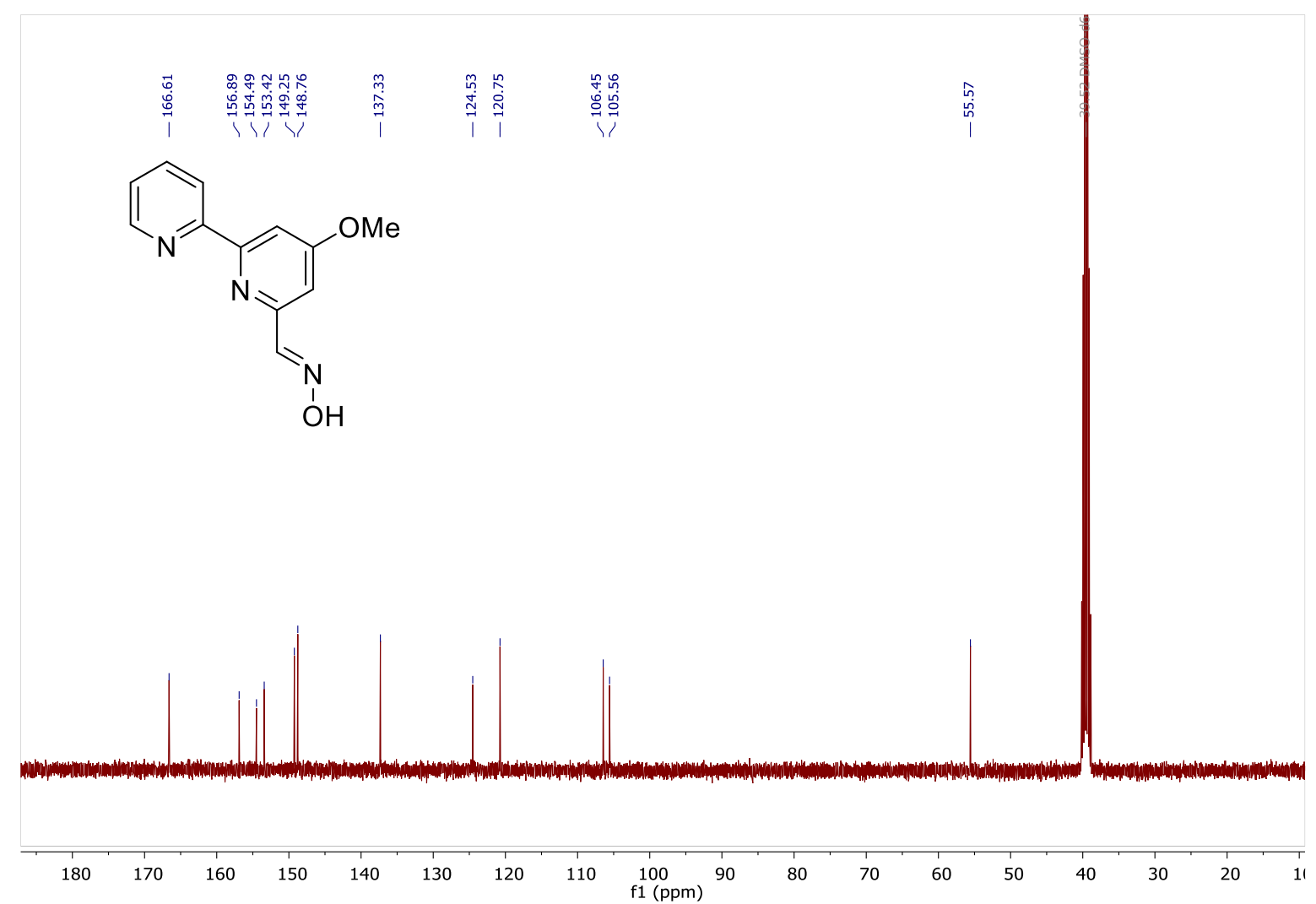




\section{References}

(1) Petersen, T. B.; Khan, R.; Olofsson, B. Metal-Free Synthesis of Aryl Esters from Carboxylic Acids and Diaryliodonium Salts. Org. Lett. 2011, 13, 3462-3465. https://doi.org/10.1021/ol2012082.

(2) Duong, V. K.; Horan, A. M.; McGarrigle, E. M. Synthesis of Pyridylsulfonium Salts and Their Application in the Formation of Functionalized Bipyridines. Org. Lett. 2020, 22, 8451-8457. https://doi.org/10.1021/acs.orglett.0c03048.

(3) Rohbogner, C. J.; Wagner, A. J.; Clososki, G. C.; Knochel, P. Magnesiation of weakly activated arenes using $\mathrm{Tmp}_{2} \mathrm{MgCl}$.2LiCl: Synthesis of tert-butyl ethyl phthalate. Org. Synth. 2009, 86, 374. https://doi.org/10.15227/orgsyn.086.0374.

(4) Watson, S. C.; Eastham, J. F. Colored Indicators for Simple Direct Titration of Magnesium and Lithium Reagents. J. Organomet. Chem. 1967, 9, 165-168.

(5) Arisawa, M.; Tazawa, T.; Tanii, S.; Horiuchi, K.; Yamaguchi, M. Rhodium-Catalyzed Synthesis of Unsymmetric Di(Heteroaryl) Sulfides Using Heteroaryl Ethers and S-Heteroaryl Thioesters via Heteroarylthio Exchange. J. Org. Chem. 2017, 82, 804-810. https://doi.org/10.1021/acs.joc.6b02585.

(6) Markovic, T.; Rocke, B. N.; Blakemore, D. C.; Mascitti, V.; Willis, M. C. Pyridine Sulfinates as General Nucleophilic Coupling Partners in Palladium-Catalyzed Cross-Coupling Reactions with Aryl Halides. Chem. Sci. 2017, 8, 4437-4442. https://doi.org/10.1039/c7sc00675f.

(7) Gao, G.; Xia, W.; Jain, P.; Yu, J.-Q. Pd(II)-Catalyzed C3-Selective Arylation of Pyridine with (Hetero)Arenes. Org. Lett. 2016, 18, 744-747. https://doi.org/10.1021/acs.orglett.5b03712.

(8) Bergmann, A. M.; Oldham, A. M.; You, W.; Brown, M. K. Copper-Catalyzed Cross-Coupling of Aryl-, Primary Alkyl-, and Secondary Alkylboranes with Heteroaryl Bromides. Chem. Commun. 2018, 54, 5381-5384. https://doi.org/10.1039/c8cc03145b.

(9) Hickey, D. P.; Sandford, C.; Rhodes, Z.; Gensch, T.; Fries, L. R.; Sigman, M. S.; Minteer, S. D. Investigating the Role of Ligand Electronics on Stabilizing Electrocatalytically Relevant LowValent Co(I) Intermediates. $J . \quad A m$. Chem. Soc. 2019, 141, 1382-1392. https://doi.org/10.1021/jacs.8b12634.

(10) Zhou, M.; Tsien, J.; Qin, T. Sulfur(IV)-Mediated Unsymmetrical Heterocycle Cross-Couplings. Angew. Chem. Int. Ed. 2020, 59, 7372-7376. https://doi.org/10.1002/anie.201915425.

(11) Kim, S. H.; Rieke, R. D. 2-Pyridyl and 3-Pyridylzinc Bromides: Direct Preparation and Coupling Reaction. Tetrahedron 2010, 66, 3135-3146. https://doi.org/10.1016/j.tet.2010.02.061. 
(12) Simkovsky, N. M.; Ermann, M.; Roberts, S. M.; Parry, D. M.; Baxter, A. D. Some Regioselective Cross-Coupling Reactions of Halopyridines and Halopyrimidines. J. Chem. Soc. Perkin 1 2002, 16, 1847-1849. https://doi.org/10.1039/b205027g.

(13) Chen, X.; Zhou, L.; Li, Y.; Xie, T.; Zhou, S. Synthesis of Heteroaryl Compounds through CrossCoupling Reaction of Aryl Bromides or Benzyl Halides with Thienyl and Pyridyl Aluminum Reagents. J. Org. Chem. 2014, 79, 230-239. https://doi.org/10.1021/jo4024123.

(14) Truong, T.; Mesgar, M.; Le, K. K. A.; Daugulis, O. General Method for Functionalized Polyaryl Synthesis via Aryne Intermediates. J. Am. Chem. Soc. 2014, 136, 8568-8576. https://doi.org/10.1021/ja504886x.

(15) Molander, G. A.; Canturk, B.; Kennedy, L. E. Scope of the Suzuki-Miyaura Cross-Coupling Reactions of Potassium Heteroaryltrifluoroborates. J. Org. Chem. 2009, 74, 973-980. https://doi.org/10.1021/jo802590b.

(16) Chen, W. C.; Hsu, Y. C.; Shih, W. C.; Lee, C. Y.; Chuang, W. H.; Tsai, Y. F.; Chen, P. P. Y.; Ong, T. G. Metal-Free Arylation of Benzene and Pyridine Promoted by Amino-Linked Nitrogen Heterocyclic Carbenes. Chem. Commun. 2012, 48, 6702-6704. https://doi.org/10.1039/c2cc32519e.

(17) Trecourt, F.; Gervais, B.; Mongin, O.; Gal, C. Le; Mongin, F.; Queguiner, G. First Syntheses of Caerulomycin E and Collismycins A and C . A New Synthesis of Caerulomycin A. J. Org. Chem. 1998, 63, 2892-2897. https://doi.org/10.1021/jo972022i. 